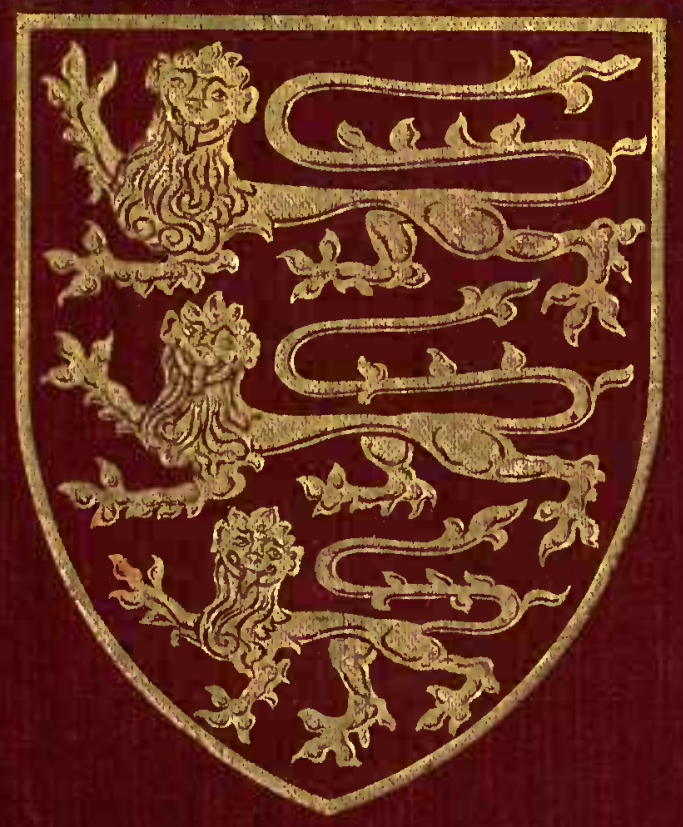

DA

670

B3V 6

Index

ROBA 


The Victoria Ibistorn of the Countíes of England

EDITED BY WILLIAM PAGE, F.S.A.

\author{
A HISTORY OF \\ THE COUNTY OF \\ BEDFORD
}

I N D E X 



\section{INDEX TO THE VICTORIA HISTORY OF THE COUNTY OF BEDFORD}
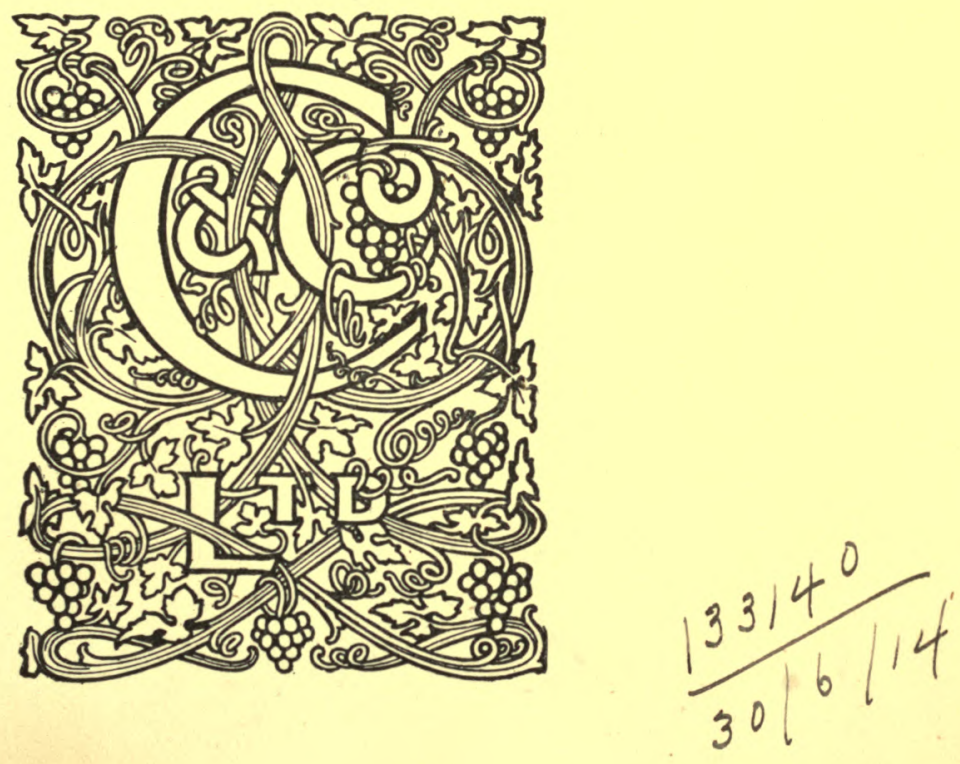

\section{LONDON}

CONSTABLE AND COMPANY LIMITED

$$
\text { I } 9 \text { I } 4
$$




$$
\begin{aligned}
& \text { DA } \\
& 670 \\
& \text { B3V6 } \\
& \text { Index }
\end{aligned}
$$


CONTENTS OF INDEX VOLUME

Contents

Note.

General Index

Corrigenda and Addenda

List of Subscribers 


\section{THE VICTORIA HISTORY OF THE COUNTIES OF ENGLAND}

The publication of the volumes of this series which form the history of the county of Bedford is due to the financial support afforded by the Right Hon. the Viscount Hambleden, the Right Hon. the Viscount Alverstone, G.C.M.G., the Right Hon. the Lord Ashcombe, Mr. O. E. D'Avigdor Goldsmid, D.L., Mr. Somerset A. Beaumont, D.L., and the late Mr. Frank McClean, F.R.A.S., of whose public spirit and generosity it is here desired to make special recognition. 



\section{GENERAL INDEX}

Note.-The Domesday Introduction and Translation of the Domesday Text are indexed separately in Vol. I. The articles on Geology, Palaeontology, Botany and Zoology being usually in the form of lists are not indexed, The following less obvious abbreviations are used:-adv., advowson; b., brother; cast., castle; chant., chantry; chap., chapel ; coll., college ; ct., court ; ctss., countess; d., daughter ; dchss., duchess ; dk., duke ; D. and C., Dean and Chapter; f., father; hund., hundred; ind., industries; man., manor ; mchnss., marchioness; m., mother; mon., monastery; par., parish; pop., population; sch., school ; sis., sister; sts., streets; vsct., viscount; vsctss., viscountess; w., wife.

Aaron, iii, 2, 363

Abbas, iii, 23

Abbesse, Will., iii, 25 I

A bbey Close (Bedford), iii, 6

Abbey Farm (Renhold), iii, 2 I 5

Abbot, Chas., ii, I 73 ; Rev. Chas., i, $37,4^{1-50}, 5^{2}, 54-6,58-63,79$; Maurice, iii, 325 ; Sir Rich., ii, 59 ; Rob., iii, 325

Abbots Lands, ii, 383

A bbots Langley (Herts.), ch., ii, $3^{I} 7$

Abbotsley (Hunts.), ii, 7 I

Abergavenny, Geo., Lord, iii, 229 ; Jno. de Hastings, Lord, iii, 229, 233

Abernon, see D'Abernon

Abingdon (Berks), ii, 48

A bingdon A bbey, ii, 2 I 1 ; iii, 345

Abraham the Jew, iii, 29I, $3^{83}$

Accleia, see Oakley

Achelai, see Oakley

Achi, ii, 339

Ackroyd, Rawson, iii, 132

Ackworth see Acworth

Aclai, see Oakley

Acton Burnell, iii, 7I

Acworth, Frances, i1, 388; Geo., ii, $39,360,362,363$; iii, 1 26, 203 , 386; John, ii, $352,373,374$, 380 ; Rich., iii, 203, 204 ; Will., ii, 362

Adam (vicar of Oakley), iii, 333; the clerk, iii, 23

Adams, Agnes, iii, 5o; J. Reynal, iii, 384 ; Mrs. J. W., ii, 222 ; Rob., ii, 335 ; Theo., ii, 335 ; iii, 432 ; Thos., iii, 50, 104; Will., ii, $4^{\circ}$

Addington, Gt. (Northants), i, I89

Addington, Rev. Hen., ii, 282 ; Joseph, iii, 206, 209 ; J., i, I 23 ; Luke, ii, 255 ; P., i, IOO, IOI, I10, I 16, I 19, I 20, I22, I25, I26, 128, I 30,139 ; Thos. A., ii, 282 ; W., i, 132 ; W. J., ii, rgo

Adelitha, iii, 426

Adeliza, iii, 240

Adelmare, Cesar, ii, I 59

Adelold, iii, ro I

Adey, Dan. Goodson, ii, 3I7, 319

Adingrave, Alice de, iii, 384 :

Eleanor, iii, 292 ; Ellen de, iii, 384 : Hen. de, iii, 384 ; Jno. de, iii, 384 ; Thos., iii, 2 I $7 n, 384$

Adingrave's Close (Renhold), iii, 2 I 7

Ad Pontes, ii, 4

Adrian IV, pope, i, $359 n$

Adser the Black, iii, $40 \mathrm{I}$

Adye, Rev. Fran. W., ii, 3I 7, 3 I9

Adyngraves, see Adingrave

AEdgiva, ii, 207

Ælfgifu, iii, 345

Elfhelm, ii, 237 ; iii, I 50
Ælfleda, iii, I24

Elfric, iii, 128

Æthelwine, ii, I9, 20, 2

Affa, w. of Alfhelm, ii, 23

Agricultural Machinery Works, ii, I 25, I 37

Agricultural Organization Soc., ii, 142

Agriculture, ii, r $08-9$, 129-42

Agylon, Agnes, ii, $234 n$; Rob., ii, $234 n$

Aickin, Eleanor, iii, I33 ; Fran., iii, I 33

Aieworde, see Eyworth

Aiken, Pet., iii, $72 n$

Ailesbury, earl of, ii, $57,58,60,6 \mathrm{r}$, 63,267 ; iii, I9, 296, 36r, 362 : Rob., earl of, iii, $272,290 n, 292$, $317,324,325$; Thos., earl of, ii, 59 ; iii, 27 I, 31 4, 3I 5

Ailey, Chas., ii, 190

Ailey Green, i, 29

Ailwin, ii, 294

Aisseworde, see Eyworth

Akeman Street, ii, 5

Akle, see Oakley

Alava, archd. of, iii, 327

Alban, St., arms, ii, 368

Albemarle, ctss. of, iii, I 30, 395, 398; Hawis, ctss. of, iii, $284,285,286$; Isabel, ctss. of, iii, 285 ; earls of, ii, 28 ; iii, 29, 395 ; Bald., ii, 350 iii, 285 ; Will., iii, $284,285,286$

Alberd, Amieia, ii, 287; Amy (Anne), iii, 429; Lucy, ii, 287 ; iii, 429; Reginald, ii, 8I ; Rich., ii, $8 \mathrm{I}$ Will., ii, 287 ; iii, 429

Alberic, cnt., see Vere, Aubrey de

Albingi, see Daubeney

Albini, see Daubeney

Albone, Dan, ii, I27

Albreda, iii, I 75

Albyn, Rob., iii, 60

Aldeburgh, Rich. de, iii, I7

Alderman, Robt., iii, 57.

Aldermanbury Manor, iii, 23

Aldgate, Friars Minor, ii, 239: Nuns Minoresses, i, $326 n$

Aldridge, Amb., iii, 449

Alee, Agnes, iii, 366 ; Eliz., iii, 366 ; Rob., iii, 366

Alevold, iii, 78

Alexander III, pope, i, $3 I_{4} n, 353 n$, 378,388 ; ii, I 54, 209, 309, 312, 332,380 ; iii, 127, 1 43, I 79, 276 278

Alexander, E., iii, 426,428 ; Jos. T. B., iii, $60,76,80$; see Lincoln, bps. of

Alexandra, Queen, i, roo

Aley Green, ii, 314

Aleyn, Hen., iii, 230; Jno., iii, 230, 231 ; Kath., iii, 230 ; see also Allen
Alfelinus, see Elfhelm

Alfhelm, see Æifhelm

Alford, Lady, ii, I $85 n$; Jno., vsct. ii, $66 n$; Sir Edw., ii, 212

Alfred, King, i, 269, 276, 297, 310: ii, 18,73 ; iii, 345

Alfwold, iii, $33^{8}$

A]gae, i, 59-60

Algar, iii, 89, 41 8

Aliams Fee (Totternhoe), iii, 448, 449

Alington, C., ii, 197 ; Jno., ii, 207, 30I ; Julius, ii, 206, 207, 30I iii, 3Io; Sara, ii, 207, 30I ; Will. ii, 207 ; Rev. Will., ii, 207, 30r

Allanson, Jno., ii, 165

Allcroft, John d', iii, 304

Allen, Eliz., ii, 337; Rev. Hunter B., iii, I89; Jno., iii, 319 ; J., i, I27; P., ii, I99; Will., ii, 337 ; -, i, 135; iii, 317 ; see also Aleyn

Allen, W. H., Son \& Co., ii, I26

Alley, Fran, ii, 362 ; Jno., ii, 362

Alli, iii, 52, 77

Allic, iii, I 38

Allington Hill, i, I 16

All Souls Coll. (Oxf.), iii, 424

Almar, i, 300 ; ii, 215 ; iii, 38,92

Alneto, Alice de, iii, r Io; Hen. de, iii, I $4 n$; Hugh de, iii, rro, 200 ; Sarah de, iii, I Io, III; Will. de, iii, I10, I 16 ; fam., iii, I I 3

Alnthorpe, Alice, ii, 322 ; Walt., ii, 322

Alnutt, Alf., iii, 338

Alric, ii, 282 ; iii, 258, 26o, 459

Alsey, Will. de, iii, 9 I $n$

Alsi, iii, 37, 44, 77; see also Ulsi

Alston, Alex. R., iii, 90 ; Andr., ii 299 ; Anne, iii, 66; A. R., iii, 88; Sir Crewe, iii, 73 ; Sir Edw. iii, 119; Eliz., iii, 73; Frances, iii, 76; Jno., iii, 78, 83; Sir Justinian, iii, 73 ; Justinian, iii, 77 ; Sir Rowland, ii, 62, $66 n$; iii, 50; Rowland C., iii, 50, 73, 77, I 02 ; Temperance, iii, $73 n$; Sir Thos., ii, 44, $63 n$; iii, 5 I $72 n, 73$; Thos., ii, $66 n$; iii, 73 , 77,83 ; Vere J., iii, 76 ; Sir Will., iii, 74 ; Will., iii, $73,75,76,77$, 78,83 , ro2 ; Capt., ii, 70 ; fam., ii, 44,66 ; iii, 72

Altham, Alicia Annesley, Lady, iii, 4 II

Althorpe, iii, IO2

Alton (Hants), ii, 3

Alvric, ii, 278, 321, 322, 329; the priest, iii, 159

Alwey, Anne, ii, 354 ; Dorothy, ii, $354^{\text {; }}$ Mary, ii, 354 ; Ralph, ii, 354 Alwin, ii, 224, 248, $267 n, 277$. 753 ; iii, I I 4, I 37, 2 I I, 276, 3 I 4 344 


\section{A HISTORY OF BEDFORDSHIRE}

Alwold, iii, 74, 84

Alwyn, see Alwin

Amaury, Edm. de, iii, 384 ; Sir John, iii, 384 ; Jno. de, iii, 384

Ambrose, iii, 433

Amersham, Jno. of, $i, 38$

Ames, Levi, ii, 374; Lionel, ii, 357, 374

Amesbury Priory (Wilts), i, 404: iii, 403

Amethella, see Ampthill

Amphibal, St., arms of, ii, 368

Ampthill, $i, \mathbf{1} 45,324,336 n, 337,346$, 367 ; ii, $36,38,39,40,44,46,55$, $56,66,67,102$, I I $5,133,195$, $266,293,320,323,376,381$; iii $197,267,268,289,313,320,455$; adv., iii, 273; 'Alameda,' iii, 269; almshouses, iii, 268, 269, 270 castle of, ii, I 47 ; iii, 270 ; char., iii, 274,275 ; Chas. I at, ii, 4 I ; ch., i, $315 n, 333,334,335 n$, 344,352 ; iii, 268, 273 ; clocktower, iii, 268 ; cross, iii, 270 ; deanery of i, 347 ; 'Duke's Cottages, 'iii, 269 ; hosp., iii, 275 . ind., iii, 268; inns in, iii, 268; man., ii, $95,96,147,308,327$; iii, 270 , $271,272,285,361$; mkts. and fairs, ii, 88 ; iii, 268, 269, 27I, 272 ; moot-hall, iii, 268 ; pks. iii, 270, 272, 292 ; Queen Kath. at, ii, I22, 123 ; iii, 35.3 ; rectory, iii, 273, 333 ; Rom. rem., ii, 4 ; schs. ii, I8I ; vicarage, i, $316 n$

Ampthill, hon., i, 357: ii, 39, I45, I 47, 267, 295, 321, 322, 376; iii, 82, 205, 25I, 252, 27I, 272 $276,281,285,287,290,292,294$ $301,306,314,317,318,321,324$, $325,326,331,339,347,361,376$, 396,4 I8, 4I9, 442, 443, 452, 459

Ampthill; Emily, Lady, iii, $27^{\circ}$; Ld. iii, I44, I49 ; Arth., Ld., ii, 66

Ampthil, Jno., i, 384

Ampthill Fields (Maulden), iii, 316

Ampthill House, i, 122 ; ii, 61 ; iii, 270

Ampthill Moor, i, 50

Ampthill Park, i, 7, 65, 108, 126 ii, $61,147,197$

Ampthill Woods, i, 63

Anabaptists, i, 344 ; iii, 432

Anable, Will., iii, I 45, 366

Ancaster, duke of, ii, I9I

Anderson, Awdry, Lady, ii 233 Cath., ii, 233; Sir Edm., ii, 2 II, 231, 232, 233 ; iii, 106, 452 ; Edm., ii, 231,233 ; Eliz., iii, 452 ; Sir Fran., ii, 233; Fran., ii, 23I; Judith, ii, 23I, 233 ; J.' O., ii, I99: Magdalen, Lady, ii, 23I, 233: Marg., ii, 233 ; Mary, ii, 233 ; R. E. H., ii, 200 ; Simpson, ii, 277 : Sir Steph., ii, 23I, 233; Steph., ii, 23 I

Andrew, Ann, iii, 222; Benj., ii, 320 ; Bern., i, 399

Andrews, Benj., ii, 317 ; Joan, ii, 3I 7 ; Jno., ii, 262 ; Jonas, iii, 33 ; Rich., ii, 347 ; Will., ii, 262

Angell, Helen, ii, 382 n: Thos., ii, $30 I$

Anglo-Danish remains, i, 282

Anglo-Saxon burials, i, I 75, I77, 184,185 ; cemetery, i, 176, 186, I87; iii, 297, 402 ; remains, i, I75: ii, 8, I I, 17, 242 ; iii, 57,297 , 439 ; weapons, i, $177,182,183$, $190,285 n$

Anne (of Cleves), Queen, iii, 27r : (of Denmark), Queen, ii, 210 , 295, 297, 339 ; iii, 2 I9, 276; (w. Rich. II), Queen, iii, I6
Annesley, Alicia, see Altham, Lady: Joan, iii, I7o; Jno., iii, I70;

Will, iii, 82

Annestey, Jno., iii, $3^{85}$

Anscell, see Ansel!

Anschetil, see Aschil

Ansell, Edw., ii, 28I; Eliz., iii,

I 84; Thos., ii, 323 ; iii, I84, I95

Anselm, i, 37 I

Ansfrid, iii, 37

Anstey, Eliz., iii, I50 ; Sir Hen., ii, $41 ;-$, ii, 363

Anthony, fam., see Antonie

Anti, iii, 4 I 8

Antonie, Jno., iii, 92 ; Lee, ii, 65 r 32, I87; Mark, iii, 88, 92 . Rich., iii, 92

Apothecaries' Soc., Gideon de Lanie (Delaune), master of, iii, 2 I 9

Appelond, Jno., i, 397

Applewood, ii, 341, $34^{2}$

Appley Corner (Haynes), ii, 338, 342

Apprentices, iii, 18

Aprice, Edm., ii, 346; Isaac, ii, 346 ; Jane, ii, 346 ; Jno., ii, 346 ;
Rob., ii, 346 ; Will., ii, 346 ; 一, i, $363 ;$ iii, $4^{8}$

Ap Rys, see Aprice

A psley Green, iii, 296

Apthorp (Apthorpe), E. E., ii, 199 :$$
\text { Steph., ii, 28o }
$$

Archdale, G., ii, I90

Archer, Álice, iii, 50; Gilb., iii, 376 Nich., i, $384 n$; Rich., iii, 50 Rev. Thos., i. $336 n$; ii, 325 ; iii, $289 n, 290,295,296$; Tim., ii, 292 ; Dr., iii, 233

Archiaco, Aymer de, ii, 351, $352 n$; Mabel de, ii, 35 I

Ardens, fam., see Ardern

Ardens, man., see Arderns

Ardern, Eustacia de, iii, 220 ; Nichola, iii, 84 ; Rob. de, iii, 84 ; Thos. de, iii, 220, 221 ; Will., ii $271,342 n, 343,344$

Arderns, man. (Roxton), iii, 220

Ardren, see Ardern

Ardres, man. (Turvey), iii, I IO, I 12

Ardres, Baldwin des, see Guines, count of ; Ernulf de, iii, 46, 74 78, IoI, I I : Hugh de, iii, II 2 : Isolda de, iii, I12; Jno. de, iii, I 2 ; Rich. de, iii, I 12 ; Rob. de iii, IIO, III, II ; Sarah, see Alneto: Thos. de, iii, I I 2

Ardys, Dorothy, iii, 216 ; Jno., iii 216 ; Thos., iii, 2 I6

Argentein, see Argentine

Argentine, Dav., iii, I59; Joan, ii, 2I 5, 216; John, ii, 2I 5, 2 I6 Margery, iii, 283 ; Matilda de, iii, 3ro; Rich. de, iii, 3 Io

Aris, Eliz., iii, 157 : Jos., iii, 157

Arkwright, A., iii, 88; Esme, ii, I89; Rob., ii, I80

Arlesey, i, I 73-4, 278, 312 ; ii, 234 $260,262,263,270,300,324$ adv., ii, 265 ; char., ji, 265 ; ch. i, $313,324 n, 348 n$; ii, 26I $263,264,265$; coins, ii, $26 \mathrm{I}$ earthworks, ii, $26 \mathrm{I}$; ind., ii, $26 \mathrm{I}$ man., ii, 207, 223; mkts. and fairs, ii, 88,262 ; mills, ii, 263 ; Nonconf., ii, 265 ; pottery (Samian), ii, 4 ; rectory, ii, 265 ; sch., ii, I8I ; vicar, i, 342 ; vicarage, ii, 206, 316; wkhouse., ii, IO4

Arlesey Bury, ii, 26r, 263, 342 ; man., ii, 262, 263, 265; mills, ii, 262

Arlesey Siding, sch., ii, I8 I

Armada, it, 40

Armington, Will., iii, I I 2

Armstrong, A. A., iii, 297
Arnald, see Arnold

Arnesby (Leics.), i, 382, 384

Amold, Edw., ii, 236 ; Grey, ii,

295: Hen., ii, 9 I ; Jno., iii, I85: Kath., jii, 382 ; Marg., ii, 295 ; iii, 380 ; Mich., iii, 3 Io

Arrouasians, $i, \quad 349, \quad 3^{87} n, 388$, $388 n$

Arschil, see Aschil

Arthorw, Jno., iii, I54: Marg., iii, I 54

Artificial stone ind., ii, 127

Arundel, earl of, iii, I99

Aschil, ii, $300,38 \mathrm{I}$; iii, I8I, I 84 I 86, 262, 344, 381, 383

Asgar, ii, $36 \mathrm{I}$

Ashbroolfield (Wootton), iii, 33 I $n$

Ashburnham, Sophia, ctss. of, iii 132 ; earls of, iii, 22 ; Bert., iii, I 30 ; Geo., iii, 130, 132 n: Jno. iii, I 30 ; Eliz., Lady, iii, 126 ; Kath., Lady, iii, rзo ; Lds., iii, 270, 3 II ; Jno., iii, 322 ; Will., iii, 126, I30

Ashburnham, Jno., ii, 51, 52, 6I ; iii, $270,272,322,323$; fam., iii, I32

Ashby, prior of, iii, $1_{4} 8 n$

Ashby, Dav. de, ii, 357, 366: Isabel of, i, 360 ; Jno. of, i, 370 ; Rob. de, iii, 30

Ashcroft, - , ii, I68

Asheldon Close (Marston Moretaine), iii, 3 I I

Ashfield, Florence, ii, 280: Geo., ii, 296 ; Margery, ii, 296 ; Patience iii, 361 ; Rich., iii, 361 ; Rob., ii, 296; Thos., iii, 302 ; - iii, 305

Ashley, Alice, iii, I9I ; Eliz., iii, 207 ; Gilb., iii, 207 ; Hen., iii, 19 I Ashridge, iii, 362

Ashridge Coll. (Bucks), iii, 448

Ashton, Sir Edw., ii, 43 ; Frances, ii, I79; iii, 35 I, 367,368 ; Mary, iii, 436 ; Matth., ii, 298, 299 ; Nich., iii, 272, 437 ; Pet., ii, 299 : Rob., iii, 436 ; Will., iii, 436 $43^{8}$

Ashwell, Jno., i, 38o, $3^{81}$; Rich., ii, 38

Ashwell Street, i, I 76

Askyllus (Anskyldus), ii, 315

Aspedon (Kent), ii, 256 n

Aspin, Anne, iii, 91 ; Jno., iii, 9 I

Aspinall, Jas., iii, 304 ; Nich., ii, I67, I68, I69

Aspley, Alice de, ii, 337 ; Nich. de, ii, 337 : Rog. de, ii, 337

Aspley Bury, man., ii, 294, 295, 296

Aspley End, ii, 295 n

Aspley Guise, i, $372 n$; ii, 9I, 96, II 4 , I99, 329 ; iii, 276 , $336,338,342,394,398$; adv., iii, 339,342 ; chants., iii, 342 ; char., iii, 343 ; ch., i, 3I 5, 37 I $n$, $378,379,380$; iii, $338,340,342$; Glebe Farm, iii, $33^{8}$; ind., iii, 338 ; man., iii, 338 ; mkt. and fairs, ii, 88 ; iii, 340 ; mills, iii, 340; Nonconf., iii, $33^{8}$; Old House, The, iii, $33^{8}$; rectory, iii, 342 ; sch., ii, I 81 ; iii, $33^{8}$

Aspley Heath, ii, I8I

Aspley House (Áspley Guise), iii, $33^{8}$

Aspley Woods, , 42, 52, 56, 58, 79, Io 8; iii, 338

Asplion, Kath., ii, 257

Assandun, battle of, ii, 20

Asscheton, see Ashton

Assheton, see Ashton

Astell, Edm., ii, $356 n$ Eliz., iii, 23I ; Jno., ii, 228; Rich., ii, 228 ; iii, 231 ; Will. ii, $66 n$, 


\section{INDEX}

Astell (cont.)

228 ; Will. H., ii, 228 ; Will. T., ii, 228 ; fam., ii, 66

Astewood, see Astwood

Aston Bury, man., ii, 315

Aston Flamville, iii, 2 I6n

Astrey, Alice, ii, $295 n$; iii, 293. 300 ; Dorothy, iii, $382,438,446$; Fran., iii, 187, 442 ; Geo., iii., I93; Sir Hen., iii, 442 ; Hen., iii, 376 , 38o, 442 ; Jas., iii, 442 ; Lora, iii, 193; Mary, ii, 338 ; iii, 193, 380 ; Ralph, ii, $40 n, 295 n$; iii, I46, 293, 300, 380, 442, 454 ; Rich., iii, 442 ; Susanna, iii, 382 ; Will., iii, 442 ; fam., iii, 379

Astwick, ii, 96, 201, 203, 205, 248, $28 \mathrm{r}, 29 \mathrm{r}$; iii, $69,96 n$; adv., ii, 203, 206; chap., i, $314 n, 315 n$, 3I8, 392 : char., ii, 206 ; ch., ii, 203, 205, 206 ; Ch. Farm, ii, 203 ; man., ii, 203, 204, 205, 262 ; iii, 44, 77; mills, ii, 203, 204; Samian pottery, ii, 4; Rom. Brit. weapons, ii, 4 I ; rectory, ii, 206

Astwick, Elias of (de), i, 31 $4 n$; ii, 203 ; Jno. of (de), i, 379, 381 ; ii, 203, 204, 289: Walt. de, ii, 203 ; Will. (de), ii, 203, 204, 205 ;

Rich. de, ii, 203; Sim. de, ii, 203

Astwick Bury, ii, 203

Astwick Mills, i, 142

Astwood, ii, I87; iii, I 16

Astwood, Jno., iii, 428 ; Nich. iii, 30

Asty Wood (Stagsden), iii, 96

Athall, Will., i, 398

Athelgyth, iii, 431

Athelney, i, 276

Athelstan, King, ii, 3 I $_{4} n, 350 n$; iii, 345

Athol, Dav., earl of, iii, 3, 28I

Atkins, Annabella, iii, I33; Edw. iii, I93, 296 ; Sir Hen., iii, 133; Thos., iii, I33; Will., iii, 246 ; Lady, ii, 42

Atkinson, Alex., ii, 24 ; C. B., ii, I99; Thos., iii, 201

Atoun, Jno., i, 393

Attelburgh, Sibyl, i, 360

Atterton, Jno., iii, 25 I

Atwood, Agnes, ii, $363 n$; Thos., ii, $363 n, 373,375$

Aubervill, hon. of, iii, 377, 4 I9

Aubervill, Will. de, iii, 377, 4 I9

Auckland, canon of, i, 327

Audele, see Audley

Audley, Alice, iii, r92 ; Anna, iii, 295; Hen., ii, 342, 344, 353; iii, I32, 1 47, 408; Jas. de, iii, 299; Rob., ii, 43; Sir Thos., iii, I3 : Thos., iii, 295 ; Will, ii, 40 ; ii, 44,54

Audley End, iii, I3 I

Auferney Wood, ii, 342

Augustinian Canons, see Austin Canons

Auncells (Milton Bryant), iii, 4 Ig

Aungeyn, Rog., iii, 346

Aunsell, see Ansell

Austin, Fran. M., iii, 332 ; Rob., ii 232 ; Thos. G., ii, II9; W., ii, 198

Austin Canons, i, $349,350,363 n$, 37 I-87, $388 n, 395 n$; ii, 154 iii, 2 I $n$, I 73

Austin Nuns, i, 387, 388

Austria, Jno., archdk. of, iii, 458 ; Lewis, archdk, of, iii, $45^{8}$

Avalon, Hugh of, see Lincoln, Hugh, bp. of

Aveline, iii, 285

Avenay, Rob., iii, 310

Avenel, Eliz., ii, $296 n$; Jno., ii, 296, 297 ; Juliana, ii, 296 ; Kath.,
Avenel (cont.)

ii, 219 ; Mary, ii, 219 ; Rob., ii, 296

Avery, Hen. of, ii, $289 n$; Jno., iii, 91 n: Will., iii, 368

Aves, Eliz., iii, 361 ; Rich., iii, 361

Avrenches, Matilda d', iii, 452 ;

Will. d', iii, 452

Awdley, see Audley

Axholme, Isle of, ii, 3 I

Aydrop, Thos., iii, 106, 195

Aylesbury (Bucks), ii, 46 ; iii, 383 , 393, 401, 402 ; ch., i, 3 I I ; gaol, ii, 26 ; iii, 7

Aylesbury, earls of, see Ailesbury

Aylesbury, Jno., ii, 364: Kath. iii, 77, I9I; Sir Thos., iii, I9I ; Thos., iii, 77

Aylesford (Kent), i, I 70

Aylyff, Jno., iii, 39

Aynel, Ad., ii, 360 ; Jno., ii, 360 iii, 453 ; Rog., ii, 360 ; Will., ii, 360 ; iii, 453

Aynsworth, Eliz., ii, I79 ; Sim., iii, 285,454 ; Thos., ii, 179

Azelin, iii, 236

\section{Baa, see Bathonia}

Babs, man, iii, I 46

Babyngton (Babington), Sir Will. ii, 276, 277, 280, 323; Will., ii, 276,145

Bach, Maria G., iii, 53, Thos., iii, 53

Backenho, man. (Thurleigh), i, 297 ? iii, ro6

Backnoe End Farm (Thurleigh), iii, $\mathrm{IO}_{4}$

Bacon, Sir Fran., iii, 253, 285 ; Rob., iii, 95, 106; Will., iii, 95 fam., ii, 233

Baddyngho, Joan, iii, $217 n$

Badelesdone, see Battlesden

Badlesmere, Barth. de, ii, 384,385 Giles de, ii, 384 ; Marg. de, ii 284,385

Bagley, Hen., iii, 94, I 16 ; Matth., iii, 94,175

Bagley's Spinney (Stagsden), iii, 96

Bagot, Chas., iii, 434 ; Frances, iii,

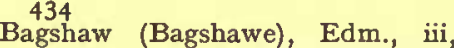
193, 326 ; Edw., iii, 193; Mary. iii, I93; Rev. R. S., ii, 285

Bailey, W., i, Ioo

Bailiff's, man. (Luton), ii, 350, 36r

Baird, Sir Dav., ii, 67

Baker, Ann, iii, 278 ; Jas., iii, 327 Joan, ii, 329, 330; Jno., ii, 39 Rev. R. S., i, 291 ; Rev. Thos., ii, I 8 I $n$; iii, I28; Thos., iii, 327 Will., ii, 329,330 ; fam., iii, 277

Baker's Wood (Leighton Buzzard), iii, 400, 405

Bakeworthe Field (Barton), ii, 309

Baldock (Herts), ii, 207, 2 I 4

Baldock (Baldok), Agnes de, ii $254 n$; Nich., i, 38I; 一 de, ii, $254 n$

Baldric, iii, 426

Baldry, Hen., iii, 400

Baldwin, the Miller, iii, 216

Baldwin, Fran., iii, I67 ; Thos., iii, 167

Baleok, Beatrice de, iii, 28 I

Baliol, see Balliol

Ball, Jno., ii, 35 ; Lucy, iii, $356 n$; Rich., iii, 356 ; Rog., iii, 2 I I

Ballard, Sam., iii, 280

Ballidon in the Peak, chap., i, $372 n$; man., i, 376

Ballington Bottom (Herts), iii 337,455
Balliol (Baliol), Clemence de, i, 354 : Devorguilla de, ii, 30,238 ; iii 297; Jno. de, ii, 30, 238 ; iii, 297; fam., ii, 30

Balliol Coll. (Oxf.), ii, 30 ; iii, 29

Balls, man., see Babs, man.

Ball's Pasture Field (Turvey), iii, II7

Bamford, Rev. Rob., ii, 209, 222 ; iii, 33

Bamforth, Rob., iii, I 8

Bamforth \& Co., ii, 127

Banbury, iii, 80

Banks, Sir Jos., ii, I 35

Bankworth, Judith, iii, 434

Baptist, Jane, iii, I83 ; Jno., iii I 83

Baptists, i, 344 ; ii, 2I 4, 222, 241 , 242, 245, 255, 270, 27I, 280, 299 $303,312,344,374,375,381,384$ 387 ; iii, $6,31,32,49,89,94$, 100 $104,109, I_{3} 6, I_{57}, 165,209,21_{4}$ $234,238,275,279,284,308,3$ I 4 $320,33^{8}, 367,389,4$ I, 4 I5, $45^{\mathrm{r}}$

Barantyn, Drew, iii, 299 ; Eliz., iii 299: Joan, iii, 299: Jno., iii, 299

Barber (Barbar, Barbor, Barbour) Agnes, ii, 316, 373: Alice, iii, 430, 449; Anne, ii, 259, 380 ; Bridg., iii, 28 ; Christian, ii, 380 . Eliz., ii, $281 n$; Geo., ii, 383 ; iii, 449 ; Hugh, ii, 162 ; Jno., ii $89 n, 281 n, 373$; iii, $2 \mathrm{I}, 28$, 293 ; Rich., i, 334 ; ii, 383 ; Rob. ii, 316 ; iii, 259, 425, 430; Rog. ii, 342 ; iii, 432 ; Thos., ii, 380 ; iii, 3 I I, 425; Will., ii, 380 ; iii, $26 I$

Bardolf (Bardolphe), Agnes, iii, 372 ; Will., ii, 30 ; iii, 372 ; fam., iii, $390 n$

Barescote, Sim., i, $3^{82}$

Baret, Hen., iii, $356 n$; Jno., iii, 257 : Rich., iii, $356 n$

Barewood (Little Staughton), iii, 165

Barford, Gt., i, 3०3, 312, $335 n$; ii, I33, I90, 280, 3I5; iii, I2: I 8 I-5; adv., iii, 185 ; bridge, iii, 3, I8I, 228 ; char., iii, 33,185 ; ch., i, 3 I $5 n, 380$; iii, I8I, I84, I85, 222 ; hall, iii, 181 ; man., ii, 207: iii, I8I, 184; mills, iii, 18I 184 ; Nonconf., iii, 181 ; rectory, i, 317 ; iii, $184 n$; sch., ii, I82; ship money, iii, 180 ; vicarage, $i$. $316 n$; iii, 18 I

Barford, Gt., hund., ii, I13 ; iii, 34, I 80, I95, I 99, 205

Barford, Little, ii, $25,44,71,72$, I97, 20I, 206-9; iii, 3Io; adv., ii, 209 ; Barford House, ii, 206 ; char., ii, 209 ; chs., ii, 206, 207 , 208, 209: man., ii, 206-8; mill, ii, 208 ; rectory, ii, 206 ; Rowe's Cottage, ii, 206

Barford, Geoff. of, iii, I84 ; Hugh of, iii, r84: Will., i, 399

Barkedich (Bed.), iii, I $n, 2$ r

Barker (Barkere), Agatha, ii, 334 Hen., ii, 334; Hugb, ii, I62 Jno. le, iii, I43 ; Rob., ii, I62 I69; Thos., ii, 335 ; iii, I 77

Barking Abbey, i, $322 n$; iii, 307 abbess, i, 312 ; iii, 306

Barle, Alianor, iii, I45 ; Jno., iii, I 45

Barleigh, Dorothy, iii, 306

Barlow, Will., bp., see Lincoln, bps. of ; - i, II9; ii, I33

Barnacke, Edm., ii, 243 ; Joan, ii 243: Jno., ii, 243; Mary, ii 243; iii, I9I; Will., ii, 243; iii I9I 


\section{A HISTORY OF BEDFORDSHIRE}

Barnard, Ellen, iii, 273 ; Jno., iii, 273; J. H., i, I27 ; ii, 195, I96, 197 : Rich., ii, 368, 369, 373; Sir Rob., ii, 64 ; iii, 20 ; Talbot, iii, 301 ; Thos., ii, $66 n$; iii, 238 ; Thos. H., iii, 297 ; fam., ii, 66 see also Bernard and Burnard

Barnardiston, Geo., iii, 245, 246 Hen., iii, 245 ; Joan, iii, 245 ; Jno., iii, 245, 246, 255 ; Marg., iii, 245 ; Sir Nath., iii, I46 ; Rob., iii, 245; Sam., iii, 146; Sir Thos., ii, 356,359 ; Thos., ii, 359 Barnes, Geo., iii, 403, 429 ; Jno., i, 336,337 ; iii, 429 ; Ralph, i, $369 n$ : Sam. E., ii, 242

Barnett, Curtis, ii, 2 I I ; 一, ii, I90, 2 II

Barnham, Fran., iii, 403, 429

Barnhard, see Barnard

Barons' War, ii, $17,27,30,31,33$, 350

Barre, Agnes de la, ii, 359 ; Eliz. ii, 256 ; Jno., ii, 256 ; Thos., ii, 256

Barringer, Will., iii, ro

Barrington, Joan, ii, 204 n

Bartows, see Burials and Burial Urns

Barr's Place (Eaton Bray), iii, 375

Barrymore, Ld., ii, 192

Bartholomew, iii, 90; Ellen, w. of, iii, 90

Bartholomew, C, iii, 90

Barthon, see Barton-in-the-Clay

Bartlow (Essex), Rom. rem., ii, 12

Barton, see Barton-in-the-Clay

Barton, Geoff. of, i, 377 ; Jas., iii, II 7 ; Louisa, iii, 117 ; Mary, iii 117 ; Dr., ii, 167 ; - , ii, 169

Barton Hills, ii, 71, 308, 344, 381

Barton-in-the-Clay, ii, $25,50,77$, $82,87,129,306,308-13,384$ iii, 59 ; adv., ii, 312 ; char., ii, 313; ch., i, 313; ii, 308, 311,312 ; ind., ii, II 7 ; man, $i, 312$; ii, 80 308-10, 313 ; mills, ii, 310; Non conf., ii, 312 ; iii, 32 ; petrifying spring at, i, 3I ; ii, 308 ; prehist. rem., i, 162, I74; ii, 308 ; rectory. ii, 308,312 ; sch., ii, 181

Barton-on-Humber, iii, I 3 I

Barwick, Pet., ii, 295

Barworth, see Barwythe

Barwythe (Studham), iii, 429 chant., i, $325 n, 326 n$; iii, 428 , 432 ; chap., i, 318 ; ch., iii, 431 ; Grove, the, iii, 430 ; man., iii, 426 Barwythe House, iii, 428

Basingham (Basingeham), Agnes (de), ii, $234 n$; iii, 70; Beatrice de, iii, 7 I $n$; Jno. de. iii, 7 I Rob. (de), ii, $234 n$; iii, 70 Walt. de, iii, 426

Baskerfield, Mary, iii, 4 I 5

Baskerville, Thos., iii, 35I, 363

Basmead, man., iii, I90, I92, 193 man. house, i, 306

Basmey, see Basmead

Basset (Bassett), Agnes, i, 398 Alianor, iii, I45 ; Fran., ii, $66 n$ Fulk, iii, 84 ; Sir Jno., iii, I45 I46; Jno., ii, 289 ; iii, 145 Juliane, i, 357 ; Kath., iii, I 45 Nich., i, 353; Norman, iii, I 45 Ralph, iii, 57, 58 ; Rich., i, 353 ii, 23; iii, 107 ; Rob., iii, I25 I 45 ; Sim., i, $398 n$; Sybil, iii 145: Thurstan, iii, ro7; Will. iii, 145, 146; fam., ii, 66 ; iii, 22

Bassets, man. (Milton Ernest), iii, 145, 146

Basseville, Alice de, i, 360

Bassingbourn(e), Kath., ii, 346 ; Mary, ii, 219 ; Thos., ii, 346 ; iii, 22 ; Warin de, ii, 219
Bates, Geo., iii, 404 ; Rev. Thos., ii, $316 n, 318 n$

Bateson, Will., ii, 385

Bates's Close (Cardington), iii, 238

Bath, Jno., earl of, ii, 336, 339 ; Thos., mqs. of, ii, $34^{\mathrm{I}}$

Bath, Agnes de, iii, 192 ; Eliz. de, iii, 192; Fulk of, ii, 238 ; Joan of, $\mathrm{ii}_{23}$ : Osbert de, iii, I92. Reg. de, iii, 192 ; see Bathonia

Bath and Wells, bp. of, ii, 38, 310 iii, 192

Bathonia, Anne de, iii, 221 ; Walt. de, iii, 22 I see Bath

Bathurst, Allen, Ld., ii, $60 n$; iii, 344,384

Batrachians, i, IO2-3

Batsford, Sampson de, iii, 74; Wm. de, iii, 74

Battams, Geo., iii, 52 ; Thos., ii, I 38 ; iii, 52

Batten, Eliz., iii, 335; Jas., iii, 335 ; Rich., iii, 335

Batthyany, count, ii, 194

Battison, Thos., ii, I72 ; iii, I7

Battlesden, ii, 96, 356; iii, 336, 343-5; adv., iii, 345; char., iii, 345,368 ; ch., iii, 343,344 , 345; Hill Farm, iii, 343; lake, i, I 36 ; man., iji, 343,344 ; pis., i, 125 ; iii, $343,400,421$; rectory, iii, 343 : sch., ii, I8I ; vicar of, iii, 345

Battlesden, Thos., i, 401

Battlesden Abbey, iii, 6

Battye, Will., iii, 437

Bawde, Jane, iii, 39r ; Thos., iii, 391

Bawdery, Will., iii, 407

Baxter, Jno. de, ii, 309 ;-, i, 343

Bay, Sim. de, iii, 85

Bayeux, man., see Ocle-cum-Clapham

Bayeux, bp. of, i, 313 ; iii, 96, I I 4 , I 26, 223,370, 376, 419, 449 n

Bayeux, Eliz. de, iii, I54: Joan de, iii, 155; Marg. de, iii, I55; Rich. de, iii, 130, 154; Rob. de, iii, I54: Sim. de, iii, I 30 ; Will. $\mathrm{de}, \mathrm{iii}, \mathrm{r}_{30}$

Bayford, ii, 3I7

Bayley, Ellen, iii, 428; Jas., iii, 310: Will., iii, 428

Bayliffe, Chas., ii, 280

Bayncroft, see Beancroft

Baynes, Mary, iii, 31, 32

Bayonne, bp. of, ii, $3^{8}$

Bayons, Will., iii, 162

Beacher, see Beecher

Beadlow Farm (Clophill), ii, 32

Beamont, see Beaumont

Beancroft (Marston Moretaine), iii, 308 ; man., iii, 3 I I

Beancroft Farm (Marston Moretaine), iii, 3 II

Beauchamp, bas., i, $300 n, 393$; iii, 61, 92, 97, 386

Beauchamp, Lord de, iii, r62

Beauchamp, Alice de, iii, I9I Ant., ii, 337 ; Beatrice de, iii, $44,46,97,114,215,235,329$, 330,383 ; Cicely de, ii, 351,352 . Edith, Lady, iii, 136 ; Edith de ii, 203 ; iii, 137 ; Ela de, ii, $30 n$ : iii, $14,44,45,46,136 n, 186$ $215,234,235,295,329,330$ : Eliz., Lady, ii, 324 ; iii, I40, 230 , 317,380 ; Eliz. de, i, $357,383 n$; ii, 248, 296, 324; iii, 295, 326, 330; Ellen de, i, 377; Emma iii, 4I ; Geoff. de, ii, 211, 324 ; Hen., ii, 339 ; iii, 41, 403 ; Hugh de, i, 297, 361, 377, 381 $n, 382 n$, $383,384,385,386,394$; ii, I 43 , $203,204,207,208,211,215$
Beauchamp (cont.)

$243,27 I, 28 I, 300,302,336$, $339,344,381$; iii, $9,36,37,40$ $42,44,61,91,96$, I07, I I 3, I 14 I 36 , I 38, I 45, I 59, I I I I I I, I 86, $190,193,197,199,203,204,205$ $206,207,210,216,234,238,240$ $248,257,258,259,260,262,266$ 291, 292, 3I5, 375, 4I8, 424 Ida de, i, 379 ; iii, 2 I 3, 235, 334 Isabel de, iii, 329 ; Joan de, ii 324 ; iii, I2, 234, 330, 331 Sir Jno., iii, 136, 137; Jno. de ii, 29, 3I, 203, 307, 339; iii I 2,4 I , 44, 45, I 90, I 96, 215,234 235, 295, 329, 351 ; Marg., iii, 41, 137; Maud de, ii, 243, 300 339 ; iii, $44,77,97,262,329$ Miles de, ii, 24,25 ; iii, 9, 24 Oliver de, i, $385 n$; iii, rgo, I93: Payn (Pain) de, i, 377, 392 ; ii $206,267,271,276,339,341$ 343 ; iii, 9, 24, I66, 240 ; Philippa ii, 248 ; iii, I90, 210 ; Ralph, ii, 208, 243, 251; iii, I9I, I99, 200-I, $221 n$; Reg. de, ii, $34 n$ Rich. de, ii, 296 ; iii, 2 I9, 403 see also St. Amand, Lord; Roais de, see Rohesia: Rob. de, iii, 9 ; Rog. de, i, $325 n, 386$; ii, 34 , $243,245,32 I$; iii, $4 I, 42,52$, 1 36, I90, I91, I96, 199, 200, 201 272: Rohesia (Rohese, Rose) de, i, 390, 392; ii, 154, 267 $271,274,276,341,343$; iii, 9 , Sim, de, i, 303, $314 n, 36 \mathrm{I}$, 37 I $n, 377,378,379,385 n$ $390 n, 391,392$; ii, 23, 24, 26 $29,154,155,203,206,218,267$ $271,276,302,338,339$; iii, 8 , $9,10,12,37,98,100,137,139$, I $59,185,203,208,210,214,235$ $236,237,242,261,266,329,334$ $342,348,37^{8}, 402$; Thos. de, i, 373 ; ii, 32 I ; Sir Walt., iii, 3 I 7 Sir Will. de, ii, 324 ; iii, I40, I87. 230, 380 ; Will. de, i, 36I, 362, $379,380,390 n, 391,392 n$; ii $27,28,29,71,203,206,243,244$ $245,248,267,271,276,296,300$ 339,34 I 343 ; iii, IO, I I, I 2, I 4 $44,52,98 n, 137,162,166,192$ 199, 203, 208, $210,213,215$ $216 n, 235,237,242,292,293$ $295,317,331,334,375,3^{8} 3$; fam., $i, 382,3^{85}, 390 n$; ii, $23,25 n$ 31; iii, 43, 52, 6I, 2 I 2, 236, 237, 342

Beaudesert (Leighton Buzzard), iii, 4 I 4

Beaufort, Hen., bp., see Lincoln bps.of; Jno., see Somerset,dk. of Lady Marg., iii, 41

Beaulieu, man., i, 351 $n, 352$

Beaulieu Priory, i, 3I3, $315 n$, $316 n, 318 n, 349,350,351,352$, $377 n, 385$; ii, $87,270,32$ I- 5 , 342,344 ; iii, $148,273,316,318$, 319,320 ; priors, i, 352

Beaumanor (Leics.), iii, 380

Beaumont, Edw, iii, 432: Hugh de, ii, 24, 39; Joan, iii, 437 ; Jno., iii, 28 ; Marg., iii, 301 ; Rob., ii, 379, see also Leicester, earl of ; Thos., iii, 437 ; Will. ii, 379 , iii, 432 ; capt., iii, 297 ; -, i, 99 ; fam., i, $3^{82}$

Bechelesworde, see Biggleswade

Becher, Jno., iii, 2 I 5: Sir Will. iii, 215, 2 I6 $n$; Will. ii, $224 n, 316$; iii, 2 I 5 ; capt., ii, 194

Beck, A. C., iii, 64,66

Beckering, John de, iii, 322

Beckerings Park (Ridgmont), ii, I 45,$147 ; \mathrm{iii}, 322$ 


\section{INDEX}

Beckerings Pk. Farm (Ridgmont), iii, 320

Beckerings Pk. Lodge Farm, iii, 322 Becket, Rob., iii, 316: Thos, i, 378 ; ii, $26,27,154$; 一, ii, 168 ; iii, 194

Beckford, Will., iii, $372,448,455$

Beckworth, Jos. F., iii, 335 ; Mary, iii, 335

Bedanforda, see Bedford

Bedcanforda, see Bedford

Bedcott, Rich., iii, 209; Sarah, iii, 209: Will., iii, 209

Beddington (Surr.), iii, 427

Bedeforda, see Bedford

Bedell, Gabriel, iii, II 3 ; Matth., iii, 304 ; Thos., iii, 259; Will., iii, 326

Bedford, ii, 23, 34, 39, 42, $54 n$, $55,56,57,59,60 n, 63,64,73$ $105,116,34^{\circ}$; iii, $\mathrm{I}-33,73, \mathrm{I} I 7$ 253; Adams Manufacturing Co. ii, 127; Agricultural Inst., iii, 320 ; Allen Inst., ii, 127; Angling Club, i, 99 ; A. S. rem, i, 176, 185 , 190 ; archdry., ii, 214 archds., $i, 313,315 n, 319,320$ $323,332,334,336 n, 341,348,378$ $384 n, 396,397$; ii, $29, \quad 152$ $154,155,213,265,270,356$ iii, $334,355,359,431$; artificial stone works, ii, 127 ; assessment ii, 153 ; iii, 1,2 ; assizes, iii, 22 7I, 276 ; bailiff, ii, 155 ; iii, 19 bedell of beggars, iii, 3,$8 ;$ bp of i, 332; Black Death, i, 328; bridge, iii, I, 4, 30, 44; bridewell, iii, 7 . chamberlain, ii, 58 ; chant., $i$, $329 n$; chaps., i, 324; iii, 6 char., ii, 153, 156, 157, I 70, I 72 , I 74, 181 ; iii, 31 , 32,33 ; Chas. I. ii, 52-53; chs., i, $107,176,28 \mathrm{r}$, 310, 3II, 311 $n, 312,315 n$ $324 n, 325 n, 326,331 n, 339$ $340,343,346 n, 347 n, 371 n, 380$ 396,$397 ; \mathrm{ii}, 92,152,153,155$ iii, 2, 4, 24, 52; coll. ch. (St. Paul's), ii, 25, I52, I53, I54, I55, $156,161,172,176,214,311-13$ $315 n, 345,349,379,380$; iii, $2,4,24,25$, II 4,236 ; Corn Exchange, ii, 176 ; Crown Building Soc., iii, 214 ; deanery, i, 347 ; Earl Cowper buildings, ii, 177 earthworks, i, 28o, 281, 282, 284 engineering works, ii, I26; electric works, ii, I26; gaols, ii, 26 55 , 103 ; iii, $1,7,8,306,361,379$ gate-houses, iii, 1,4 , 7 ; gilds, $i$, 330 ; ii, $92,155,156$; hosp., iii 6 ; ind., ii, 109, 126, 127 ; iii, 5 inns, ii, 62, 99, I70, 209, 222 ; $\mathrm{iii}$ $5,6,7,11,12,30$; ironworks, ii, 125, 126, 137; Jewry, i, 321 ; iii, 2 ; King's ditch, iii, 8 ; leper hosp., i, 349; library, iii, 4 Longholm Meadows, i, 110 ; man., iii, 23, 75, 114, 184 ; mkt. cross, iii, 2 ; mkts, and fairs, ii, 88, I05, 140, 268; iii, $\mathrm{I}, 3,2 \mathrm{I}, 24,35,44 \mathrm{I}$; mayor and corp., i, 354, 395-398 ; ii, 4I, 55 , $57,58,59,64,153,155,156,160$ $161,162,165,166,167,169,173$ iii, $3,5,16-23,29,30$; mills, 397; iii, 9, 24 ; mint, iii, 2 ; Moot Hall, iii, 2 ; Nonconf., i, 344 iii, 30,31 ; pars., iii, $1,5,6$ pk., iii, 5 ; parl. repre., ii, 34,64 ; iii, 20, 21 ; place-names, iii, 8 postal service, ii, 99; prehist. rem., i, 146, 147, 150, 161, 174 races, iii, 8 ; rebellion, iii, 3 recorder, ii, 58 ; iii, 19,155 rectors, $i, 337,338,341 n, 343$
Bedford (cont.)

ii, 164,176 ; rectories, $i, 331 n$ $339 n$; Rom. rem., ii, I, 4, 5, 8 Rupert, pr., ii, 46 ; schs., i, 42 ; ii, 109, 141, 149-85; iii, 2, 3 $5,6,9,25$; sessions, i, 369 ; iii, 159; ship-money, ii, 4I ; siege, iii, I ; tournaments, iii, 2 ; vicarages, i, 316 $n$; ii, 168; Vulcan Works, ii, 127 ; Yeomanry, ii, 70

Bedford, bar., i, 364, 377, 379 ii, 22, $26 n, 215,218,300,305$ $339,344,381$; iii, 9 , 12-1 5,40 $46,52,7$ I $n, 77,83,89,90,96-8$ 107, I I 4, 125, I 34, I 36-8, 145 $159,162,166,181,186,199,204$ 5, 2 10-12, 215-16, 236, 238, 240 $248,258,260,264,293,295$ $329-31, \quad 338-9,375, \quad 383,418$ 423 ; hon., ii, 283 ; iii, 90 , 166 , $203,206,210,216,346$

Bedford, barons of, iii, 12, 37 : Jno., iii, 77 ; Payn, i, $385 n, 390$ Isabella, ctss. of, ii, 39 ; Marg. ctss. of, ii, 277 ; Mary, ctss. of, ii, $39 n$; dchss. of, $i$, I 10, 136 Gertrude, iii, 318, 361; Jaquetta, ii, $225 n, 282$; iii, 118 ; dks., $i$, 99, 102, 103, 186; ii, $7,15,63$, $64,65,66,70,108,130,131,132$ $36,138,139,141,1_{4} 6,1_{47}, I_{7}$, $172,173,177,181 n, 183 n, 184 n$ I 85 n, I88, 189, I92, 193, I94, 197, 267; iii, II, I2, 19, 20, 23 , $30,94,95,100,102,107,139$, $140,142,149,150,159$, 191, 195. $201,203,204,205,207,210,2 \mathrm{II}$ $224,240,242,262,264,266,270$, $272,273,276,286,288,291,292$ 296, 305, 306, 307, 308, $310,31 \mathrm{I}$ $313,315,320,321,322,323,324$, $325,342,343,344,345,361,362$ $376,391,395,396,398,417,418$, $419,421,422,423,458,461,462$ : Fran., ii, 130, 135, 137, 139, 140 iii, $104,204,214,271,286,305$, 458,459 ; Geo., ii, 39, $39 n$. Hastings, ii, I 40 ; Herbrand A., ii, 140 ; iii, 104, 292,396, 459, 461 ; Jasper, ii, 39 ; Jno., ii, 39 , $62,68,135,358$; iii, 1 8, 207, 209, $286,290,314,318,324,457,461$ 462 ; Will., ii, 6r ; Wriothesley, ii $6 \mathrm{I}$; iii, 376 ; earls of, ii, 54,56 ; iii, 142, 302; Edw., iil, 143, 203 Fran., ii, 40, 42, 185 $n, 277$; iii, $203,376,459,462$; Hugh, ii, 24 , 39 ; iii, 9 ; Ingelram, ii, 39 ; Jno., ii, 39, 266; iii, 459; Will., ii, 43

Bedford, Cecilia of, iii, 215: Hen. of, i, 326; Hugh de, ii, 39 Isaac de, iii, $2 n$; Jno. (of), $i, 381$, 384 ; Martha, iii, 236 ; Matth. of, i, 384 ; Rob. of, i, 388 ; Sam., ii, $54 n$; Rev. Thos., iii, 236 ; Thos. iii, 236; Will. (of), i, 399; ii, 55

Bedford Abbey, i, 311, 377; iii, I, 6

Bedford Castle, i, 186, 281, 285, $296,320,362,364,372,378$; ii, $5,23,27-9,33$; iii, 2, I 2, 13 204,353 ; mill, iii, 23 ; siege, $i$ 382 ; ii, 24,28, I $44 n$; iii, 7,9 , IO, II, 24

Bedford Coll. (Lond.), ii, 177

Bedford-cum-Kyrkeby, see Bedford Major

Bedford Grammar Sch., ii, I49, 152-77, 199, 200

Bedford House (London), ii, $57 n$, $63 n$

Bedford Level, ii, $61 n$
Bedford Major, preb., ii, I53

Bedford Minor, preb., ii, I 53-4

Bedford St. Mary, see Bedford Major

Bedfordshire, agricultural societies, ii, I 40,142

Bedfordshire Chamber of Agriculture, ii, I40, I 42

Bedfordshire Game Protection Society, ii, 198

Bedfordshire Regt., ii, 45, 67-70 : iii, 149

Bedford Verd, see Bedford Minor

Bedingfield, Sir Edm., iii, 15; Sir Edw., iii, 296; Hen., iii, 296; Marg., iii, I 5

Bedlow, man. (Clophill), ii, 322, 325 ; fair, ii, 323 ; mill, iii, 387

Beech, Mary, iii, 328 ; Thos., iii, 326

Beecher (Beacher), Eliz., iii, 218 ; Mary, iii, 304; Sir Will., iii, 203 ; Will., iii, 218,304 ; -, ii, 262 ; fam. iii, 218

Beeches, man. (Pulloxhill), ii, 377

Beechwood Park (Hemel Hempstead), i, 116

Beedles, - , ii, I 70

Bee-keeping, ii, ${ }_{42}$

Beers, Geo., ii, 188

Beeston, i, 365 ; ii, 242 ; iii, 227 242-4, 246-8, 250 ; chant., ii, 242 ; man., i, 364 ; iii, 247 ; mill, iii, 247 ; Nonconf., ii, 246

Beeston, Hen, ii, 166, 17

Beeston-Caldecote, man., see Perots

Beeston Leys (Northill), iii, 247,251

Beetles, see Coleoptera

Beggary, hamlet, see Begwary

Beggary, Joan de, iii, I96; Rich. de, iii, 196; Rog. de, iii, I96

Begwary (Eaton Socon), iii 198 ; man., i, 305; iii, 196-7

Begwary Brook (Eaton Socon), iii, 189

Begwary Farm (Eaton Socon), iii, 190, 197

Beidforda, see Bedford

Bek, Thos., bp., i, 362 n, 401

Bekering, Jno. de, iii, 322

Bekeryng, see Beckerings Park

Bel, see Bell

Belcher, Miss, ii, I 77

Bele, see Bell

Belers, Rog. de, ii, 336

Belfield, Ant., iii, 428; Hen., iii, 428 ; Jno., iii, 428 ; Will., iii, $382,428-30,432$

Belgrave (Leics.), ii, 162

Belgrave, Marg., iii, t96 ; Rich., iii, I96

Belknappe (Belknap), Juliana, ii, 296 ; Rob., ii, 287,296

Bell (Bel, Bele, Belle), Joan, iii, 403: John (le), iii, 184, 258, 403; Rob., iii, 105, 443 ; Sim. le, iii, 257,258 ; Thos. iii, 184 : Will., iii, 220

Bellamy, Rob., ii, 40

Bellasis, Jane, ii, 345 ; Jno., ii, 345

Belle, see Bell

Bell End (Kempston), iii, 297

Bello, Jno. de, iii, 353

Bells, man., iii, I 55

Belrap, Alward, iii, 386

Belsham, Thos., iii, 9; Will., iii, 9

Belturbet, Will,, Ld., iii, I40

Belvoir, hon., iii, 107, I12, I13, I49, 426

Belvoir, Rob. de Ros, 1d. of, ii, 3 I $n$

Belvoir Castle, iii, II 2

Bendish, Hen., ii, 252 ; Will., iii, 163

Bendlowes, Will., iii, 300

Benedict, St., i, $350,355,363 n$, 367,392 


\section{A HISTORY OF BEDFORDSHIRE}

Benedictine monks, i, 349, 35I-3, $374,376 n$; iii, 403,446

Benedictine nuns, $i, 353-61$

Benery (Oakley), iii, I49

Benett, see Bennett

Benham (Ravensden), iii, 2 IO

Benington, Eliz., i, 360

Benison, Geo. T., ii, 382, 383

Bennell, Will., iii, 355

Bennets, man., ii, $350,359,36 \mathrm{r}$

Bennett (Benett, Bennet), Grace, iii, 282 ; Jno., iii, $8 n$; J., i, 123 136, I 37 ; Sim., iii, 282 ; Thos., ii, 106, 136, 137; Will,, ii, 107 . 137 ; 一, ii, I 30 ; iii, 387

Benson, Jas., iii, 208

Bentham, Rev. Jos., iii, I22, 417 ; Sam., ii, 166

Bentley, Anne, iii, 429; Jno., iii, 429: Thos., iii, 364

Beodeford, i, $310 n$

Beranforda, see Barford

Berdefeud, Jno. de, iii, $4^{\circ}$

Bereford, par., see Barford

Bereford, Agnes de, ii, 362 ; Alice de, ii, 362 ; Bald. de, ii, 360,362 ; Edm. de, ii, 362 ; Eliz., ii, 362 ; Hen. de, iii, 184; Humph. de, iii, 184 ; Joan de, ii, 362 ; Will. de, ii, 362 ; fam., iii, I8I $n$

Berell, Jno., iii, 247

Berells, man., see Buddenho

Beresford, Anne C., ii, 343

Berewode (Studham), iii, 428

Bergh, Rob. de, ii, 309

Berkeford, sec Barford

Berkeley, Isabel, Lady de, ii, $34^{\circ}$; Ld., iii, 346 ; Jas., Ld. de, ii, 340 Will., visct., ii, 30I: iii, I3; Cath. iii, 205; G. C. G., ii, $187, \mathbf{1} 88,194$; Sir Jno., ii, 5x, 52; Jno., iii, 205 ; Maurice, iii, $13 n$; fam. iii, 342

Berkford, see Barford

Berkhampstead (Herts), ii, 47, 157 317 ; iii, 358 ; cast., iii, 362

Berkley, see Berkeley

Bermondsey Priory, iii, 9, 24

Bernake, see Barnacke

Bernard, St., i, 361

Bernard, ii, 223; iii, 5

Bernard, Alice, iii, Ir6; Eleanor, ii, 286: Sir Jno. ii, 286; Marg., ii, 286; Nich., ii, 223; Odo, ii, 223; Rich., iii, 116; Rog., ii $223 n$; see also Barnard and Burnard

Berners, Mary, ii, 252

Berridge, Jno., i, 345, 346

Berrington, -, iii, 234

Berry, Dan., iii, 6r

Berryfield (Lidlington), iii, 305 Berrystead (Goldington), iii, 205

Bertona, see Barton-in-the-Clay

Bertuna, see Barton-in-the-Clay

Besebrigg (Sharnbrook), iii, 9r

Beseville, Marina de, ii, 275

Best, Ant., iii, 212 ; Hen., iii, 23 ; Mary, iii, 206

Bethune, Alice de, ii, 356 ; Bald. de, ii, $349-50,357,360$

Beton, Cicely, iii, 249, 250

Betterton, Fran. ii, 162

Bettesworth, Charlotte G., iii, 443 : Jno., ii, 357 ; Dr., ii, 357

Betts, Jno., ii, I T

Bevan (Bevin, Beywin, Beyum), Benj., iii, $322 n$; Rich., iii, 322 ; Thos., iii, 322 ; Walt., iii, 321 , 322 ; Will., iii, 320,322 ; fam., iii, 322

Bevans, man., iii, 322

Bevell (Bevill), Fran., iii, 392 ; Hen., iii, 36I; W., ii, r94

Beverley, Eliz., iii, 198; Jas., ii, 44. 323-4; iii, 201, 273 ; Rob.,
Beverley (cont.)

ii, 322, 374: Thos., iii, 198, 201 ; Will., iii, 198

Bevin, see Bevan

Beyum, Beywin, see Bevan

Bicheno, - ii, I 33

Bickenhill (Warws.), i, 360

Bickleswade, see Biggleswade

Bickley, Fran., iii, 26I ; Rich., iii, 261

Biddenham, iii, $31,34,36,46,66$, 82 , 175, 176, 210, 219 ; adv., iii, $36,37,39,40$; bridge, iii, 44 ; chants., i, $324,325 n, 329,334$; ii, 92 ; iii, 40, 43, 47, 49; char., iii, 40 ; ch., i, $326,329 n$; iii, $36,38,39$; ind., ii, 138 ; man., iii, $36,37,38,78$, т 10; mills, iii, 38,47 ; palaeolithic impl., iii, 36 ; place-names, iii, 40 ; pop., ii, II5; prebends, ii, 153; prehist. rem., i, 28, 33, 145, I 74 ; rectory, iii, 40; Rom. rem., ii, 5, 6, 7; sch., ii, I8I ; vicarage, i, $329 n$, $34 \mathrm{I}$; wells, ii, 2, 5, 7 .

Biddenham, Jno. of, i, 381 ; Will. of, $i, 3^{8} \mathbf{I}$

Biddlesden (Bucks), iii, 343 n

Bidwell (Houghton Regis), iii, 390

Biedcanforda, see Bedford

Bigg, Abigail, iii, 444; Abra., iii, 444; Agnes, iii, 444; Fran., iii, 443, 444; Jno., iii, 2or ; Mary, iii, 444 ; Sarab, iii, 444

Biggin Spinney (Roxton), i, I2I

Biggin Wood (Everton), ii, 226

Biggin Wood (Tempsford), i, 307 ; earthwork, ii, 25 I

Biggin Wood, Little (Everton), ii, 226

Biggleswade, ii, 44, $45 n, 54,95$, I16, 201, 209, 214; iii, 7, 18, $70,75 n, 202,205,227 n, 242$, 260 ; adv., ii, 214 ; agric., ii, I33; bridge, ii, 209, 210 ; boro., ii, $2 I_{2}$; chaps., ii, $2 I_{4}$; char., ii, I 79-81 $n, 214-15$; cb., i, 31 7 ; ii, 2Io, 2I3, 2I 4,217 ; coins (Brit.), i, 173; ii, 210; common, ii, 190, 209 ; dean of, $i, 347$; fishery, ii, 212 ; gilds, i, 330 ; ii, I79, 214 ; harriers, ii, I9o ; highway robbery, ii, 99 ; ind., $i, 39$; ii, 127,138 , I39, 212, 213; man., ii, 88, 2 IO-I 2, 21 4, 253 ; mkts. and fairs, ii, $88,209,210,2$ I2, 214 ; mills, ii, 2 I 2 ; moat, ii, 209 ; Nonconf., ii, $2 I_{4}$; parl. div., ii, 66,67 ; placenames, ii, 210 ; pop., ii, II 3 ; prebend., i, 324, 334, 338, 343 ; prehist. rem., i, 173, 174 ; races, ii, I9I ; rectory, i, 329; ii, 214; Rom, rem., ii, 4, 6, 209, 212 . schs., ii, I79-81, 214; sports, ii, 189, I99: vicar of, i, 329 ; vicarage, $i, 3 \mathrm{I} 7,34 \mathrm{I}$; ii, $2 \mathrm{I}_{4}$; wells, i, I3, 26

Biggleswade, hund., ii, 18, I13, $116 n, 143,201-55$

Biggleswade, Jno., i, 385

Bigland, Cornelius, ii, $183 n, 374$

Bigod (Bygod), Hugh, ii, 223; Maud, ii, 223; Rog. le, ii, 268 ; see also Norfolk, earl of

Bilenda, Hen. de, i, 372

Bilkemore, man., ii, 377,378

Bilkemore, Anastasia de, ii, 378 ; Matilda de, ii, 378 ; Sir Rob. de, ii, $337,33^{8}$; Rob. de, ii, $37^{8}$

Billenden, see Billington

Billesdon, see Billington

Billing, Walt. de, iii, 73

Billington (Gt. and Little), iii, 399 400, 402, 404; chap., i, 34I ; ii, II4; iii, 4I4, 4I7; ch., iii, 4II;
Billington (cont.)

Cock Inn, iii, 400; man., iii, 406,407 ; man. house, iii, 400 ; Nonconf., iii, 415 ; prehist. rem. i, 162 : rectory, iii, 414 ; sch., ii, I 81

Billington, Joan, iii, 406; Jno., iii, 406, 407

Billyndon, see Billington

Birchall's Wood (Eversholt), iii, 375

Birchfield, man., iii, I8I, I 82

Birchmore, i, 370 ; cb., i, 370 ; iii 462 ; man., iii, 461 ; man. house, iii, 462 ; rectory, i, 370 ; waterworks, iii, 457

Bird, Jno., ii, 344 ; Thos., iii, 22, 3II

Birds, i, 104-37

Birdshill, i, 50

Birrell, Eliz., ii, 289 ; Hen., ii, 289

Biscott (Biscot), i, 310; ii, 374; Bear's Moat, ii, 3 fo $n$; ch., i, 346 ; ii, 374; earthworks, i, 305 ; man., i. 350, 360-3 ; mill, ii, 366 ; Nonconf., ii, 374 ; springs, i, 19 vicarage, ii, 374

Biscott (Biscot), Ad. of, i, $322 n$, $395 n$; Godfrey of, ii, 356

Biscott Grange, Rom. rem., ii, 8

Biscott Place, ii, $362 n$

Bishop, Hen., iii, I20

Bissopescote, see Biscott

Bithray, Chas., iii, 52 ; Thos., iii, 52: Uriah, iii, 51

Bitlesden, see Battlesden

Blachesou, see Bletsoe

Black, Ad., ii, $357 n$; Artb., iii, 33 I

Blackborne Hall, iii, I04, I83: man., iii, I05

Blackborne Hall Farm (Thurleigh), iii, IO4

Black Death, ii, $88-90,278$; iii, 3 , $78,93,100,158,299,301,318$.

Black Friars, see Friars, Black

Black Pond (Thurleigh), i, 289

Blackwell, iii, 456

Blaigny (Blegner), Ernald de, ii. $215 n$; Thos. de, ii, $215 n$

Blake (Blak), Jex, ii, I 75 ; Steph., ii, $276 n$

Blakelandes (Wootton), iii, 33 I $n$

Blakesley (Northants), i, 394

Blankfront, man., see Potsgrove

Blankfront (Blancfront), Hen., iii, 422,423 ; Rob., iii, 422 ; Will., iii, 422 ; W. de, i, $315 n$

Blaydes, Fanny M., ii, 385 ; -, ii, 385

Bleadon (Somers.), iii, 54

Bleak Hal] (Biggleswade), ii, 209

Blechesham, see Bletsoe

Blechesho, see Bletsoe

Blegner, see Blaigny

Bletchley (Bucks), ii, 3० $n, 7$

Bletnesho, see Bletsoe

Bletsoe, i, 297 ; ii, $30,40,96$; iii, $34,40-43,59,6 \mathbf{r}, 234 n, 293$ $46 \mathrm{I}$; adv., iii, 4I, 43 ; cast., i 30I, 302 ; iii, 40,42 ; chant., iii, 43 ; chap., i, $325 n$; char., iii, 43 ; ch., i, $325 n$; iii, $40,42,43$; earthworks, i, 301, 302, 303 ; hermit, i 350 ; man., i, $325 n$; ii, $34 n$; iii. 40, 41 ; mill, iii, 42 ; pk., i, 44 ; pop., ii, 115 ; rector, iii, 43 ; sch. ii, 181 ; vicar, ii, $327 n, 336$

Bletsoe, Agnes, iii, r r9; Anne, iii, II9: Eliz., iii, II9; Will,, iii II 9,120

Blikeslane (Caddington), ii, 3 I 5

Bliss, Math. F., iii, 3 Io

Blisworth (Northants), i, 386

Blofield, Edw., iii, 286; Eliz., iii, 294 ; Giles, iii, 294 ; Mary, iii, 294 


\section{INDEX}

Blomfield (Bloomfield), Sir Arth., ii, 2 I $_{4}$; Fran., ii, 349 ; Rob., ii, 266

Bloodworth, Chas., iii, 6o ; Nich., ii, $345 n$

Bloody Battle Bridge, i, $285 n$

Bloomfield, see Blomfield

Bloomsbury Square (London), ii, $57 n$

Blosseville, Gilb. de, iii, 64,65 ; Sim. de, iii, 65

Blott, Jno., iii, 67 ; Will., iii, 67

Blounham, see Blunham

Blount, Mountjoy, see Newport, earl of

Blow, T. B., i, 43, 50

Blows Downs (Dunstable), prehist. rem., i, I 70 , I 72

Bluck Moor (Maulden), iii, 3 I 6

Bludeworth, see Bloodworth

Bluet, Will., ii, 224 n

Blundell, Alan, ii, 2 ro; Eliz., Lady, iii, 235 ; Emma, ii, 2 Io ; Sir Fran., iii, I3; Sir Geo., ii, 59 ; iii, I3, I4, 15, 235 ; Geo., ii, 44 ; Jno., ii, 2 ro, 253, $268 n, 27$ I, 275, $286 n$, 329 ; iii, 246 ; Rich., ii, 253 ; Rob., ii, $289 n$; Rog., ii, 329 ; Sim., ii, 2 10; Will., ii, 2 10; Will. N., iii, I5, 235 ; fam., iii, 247

Blundells, farm (Harlington), iii, 381

Blundells, man. (Caldecote), see Caldecote, Upper

Blundells, man. (Harlington), iii, $3^{80}, 38 I$

Blundells, man. (Silsoe), ii, 329, 330

Blunham, ii, I47, 20I, ; iii, 3I, 243 ; adv., iii, 233; char., iii, 233 ; ch., iii, 228, 231, 232, 233 ; fraternity, ii, 245,325 ; iii, 233 , $237,255,264$; gild, i, 330 ; lands in, i, 380 ; ii, 92 ; iii, 231 ; man., iii, 228, 229, 236 ; man. house, iii, 228 ; mkt., ii, 88 ; iii, 229 ; pop., ii, I 16 ; sch., ii, I 8 I

Blunham, Dan. L., i, $368 n, 369$

Blunham House (Blunham), ii I97; iii, 228

Blunham Mill, i, ror

Blunham with Moggerhanger and Chalton, iii, 227, 228-33

Blunt, Eliz., iii, 366 ; Hugh le, iii, 98 ; Jno., iii, 225, 366; Mountjoy, see Newport, earl of ; Rich., iii, 225; Will., iii, 225; 一, ii, I65

Blyth-King, Mrs., iii, 453

Bobelin, Sim., iii, $391 n$

Boderigan, Hen. de, ii, 354 ; Sibil de, ii, 354

Bodleian Library (Oxford), iii, 295

Boheme, Geo., ii, 69

Bohun, Eleanor de, iii, I 7 I ; Eliz. de, iii, I72; Frank de, ii, 351; Humph. de, see Hereford, earl of ; Jno. de, ii, $306,351,354$; iii, I7I, I75: Marg. de, iii, I75 ; Maud de, ii, $223 n$; Sybil de, ii, 351, 353, 354; Will. de, see Northampton, earl of ; fam., iii, 283

Boileau, W. H., ii, I95

Bokland, man., ii, $230 n$

Boklond, Kath. de, ii, 2 rg ; Ralph de, 219

Bold, Rev. Arth., iii, 6I ; Martha, iii, $6 \mathrm{I}$

Bole, Thos., i, 384 ; Will., iii, 240

Bolebec, Hugh de, i, 366 ; iii, 310 , 3I4, 459 ; Isabel, iii, 3 I4 ; Walt., iii, 3I 4, 459 n

Boleyn, Anne (queen), iii, I I I, I 72

Boleyn, Anne, ii, 355 ; Geoff., ii, 355 ; Thos., ii, 363
Bolingbroke, earls of, ii, $44,49,63$; ii, I9, 4I, $60 n$; Paulet, iii, 4 I I37, I77; Oliver, ii, 56 ; iii, 35 , I23, I 25, I 37, I 63, I 79, I 80, 257

Bolle, Sir Chas., iii, I96

Bolnhurst (Bulehestre, Bolehestre) ii, 56 , I I 5 ; iii, I23-8, I 43, I 49 , I 53,$223 ; \mathrm{adv}$., iii, 125, 127, 128 char., iii, I28; ch., i, 312, 314 $315 n$; iii, I24-8; earthwork, i 275; farms, iii, I24; man., iii, 124, I25, 396; moats, iii, I24: place-names, iii, 124 ; rector, ii, I 81 ; iii, I28: sch., ii, 18 I

Bolstrode, Edm, iii, 286, 288

Boltere, Joan, iii, 220 ; Rich., iii, 220

Bolton, Rowland, ii, 299

Bond, Jno., ii, I8o ; iii, 408

Bondi, the Staller, ii, 383

Boner, Julian, iii, I 54 ; Thos., iii I 54

Boniface, archbp., i, 322, 374 : pope, i, 354, 359 ; iii, r7o

Bonsey, H. D., iii, 19

Bonville, Eliz., iii, 89 ; Will., iii, 89

Boot, Will., ii, 304

Booth, Will., iii, 342

Borard, Rich. de, see Bossard

Borchard, Sim., iii, I I 2

Bord, Hen., ii, 295

Borgred, iii, I 20

Borham, Hen. de, ii, $3^{80}$

Borne, Eliz., iii, 22 ; Rich., iii, 7 22 ; Will., iii, 22, 335

Borms, Nich., iii, 285, 437

Borough, Randall, iii, 246

Boroughbridge, battle, ii, 23I, 300 , 339 ; iii, 12

Borret (Borred), iii, 9o, I33, I62, I 76

Borrett, Jno., ii, 295

Borstall, Will., iii, 244

Bosanquet, Dav., iii, 185

Bosard, see Bossar

Boscombe, Alestan of, ii, 223, 26 I

Bosgrove, Frances, iii, 216 ; Jno., iii, 2 I 6

Bosom (Bosoun, Bosum, Bosun Buzum), Alex., iii, 97, 2 r9 Anne, iii, 97; Barth., iii, 97 ; Edm., iii, 97, 98; Eliz., iii, 97 Goditha, iii, 2I9; Ida, iii, 97 Joan, iii, 97 ; Jno., iii, 97 ; Marg. iii, 219,329 ; Walt., iii, 97 ; Will. iii, 97, 219, 329; fam., iii, 97

Bosoms, man. (Stagsden), iii, 97

Bosoun, fam., see Bosom

Bossard, Giles, iii, I39; Hugh, iii I39, I42 ; Joan, iii, I39, I 50 Jno., iii, I 39 ; Rich., ii, 3 I $n$; iii I07, I49, I 50 ; Sim. de, iii, I 49

Bossington, i, I 45

Bostocke, Edm., iii, 26I

Bosun, fam., see Bosom

Boswell, Jas., iii, 257 ; Thos., iii, I 35

Bosworth (Leics.), iii, 23 I $\boldsymbol{n}$

Bosworth, Jno. of, i, 387

Botany, i, 37-67

Botecourt, see Botetourt

Boteler, Alex. le, iii, 372 ; Alice, iii, 39, 89; Anne, iii, 37, 438 Awdry, ii, 233; Eliz., iii, 40; Geo., iii, 89, 90, 92, 95; Grace, iii, 37; Helen, iii, 37,66 ; Sir Jno., iii, 89 ; Jno., iii, 89 ; Mary iii, 37,66 ; Sir Oliver, iii, 89,90 : Oliver, iii, 68 ; Sir Phil., iii, 90, 95: Sir Thos., iii, 37 ; Thos., iii, $37,46,66,372$; Ursula, iii, $3 \mathbf{r}$, 39,82 ; Sir Will., ii, 4 I, 45, $54 n$. iii, $39,40,64,66,90,94,438$ Will., ii, 58 ; iii, $37,39,40,46$
Boteler (cont.)

$68,82,89$; fam. iii, 39,266 ; see also Butler

Botelers (Bucks), man., iii, 372

Boteley, Sir Jno., iii, 3 I $_{4} n$

Botelstan, see Battlesden

Botetourt (Boteturt), Andr., iii, 390 ; Eliz., ii, 203 ; iii, I 3 ; Joan de, ii, 364 ; Jno., ii, 275 ; iii, I 3 96 ; iii, 46, 235, 330; Maud (Matilda), ii, 203, 256, 275; iii 330 ; fam., iii, I8I, $212,283,342$ Botevilein, see Butvillein

Bothe, Maud, ii, 2 Io, 2 I I, 220 : Rob., ii, 204, 2 I I, $224 n, 226$

Botlesford, Geoff. de, iii, 426 ; Jno. de, iii, 426; Rob. de, iii 426 ; Walt. de, iii, 426, 428 ; Will. de, iii, 426

Botte, Ralph, iii, 376

Bott End (Wilshamstead), iii, 328

Bott End (Wootton), iii, 329

Bottler, see Boteler

Botvileyns (Hinwick or Brayes Farm), man., iii, 58, 84

Bouchier, see Bourchier

Boughton, Edw., iii, 3or : Eliz., iii 301 : Rich., ii, 328 ; ii, 30r: Thos., ii, 328 ; Will., ii, 328 ; iii 301

Boughton End (Lidlington), i, 45, 47 ; iii, 305

Boulde, Phil., iii, 285, 288

Boulogne, hon., iii, 74, 90, ror, II I

Boulogne, Eustace, count of, iii, 46,78 , Ior ; Reg., count of, ii, 255

Boulogne, Faramus (Pharus) de, iii, $372,46 \mathrm{I}$

Boulton, Harry, ii, I 88

Bourchier, Eliz., ii, 3I6; Humph. ii, 316, 317 ; Thos., iii, 272

Bourne, i, $387 n, 388 n$; ii, 302

Bourne, Edw., ii, I67 ; iii, 27 ; Jno., iii, 311 ; Susanna, iii, 27 ; Will. ii, 39 ; iii, 23, 31 I; - ii, I66, I 7 I

Bourne End (Cranfield), iii, 275 278,329

Boveden, Nich. de., iii, 449 ; Sarah de, iii, 449

Bover, Edw., iii, $456 n$

Bovey, Sir Ralph, iii, 254

Bovil (Boweles, Bowell, Bowles Boyvill, Bueles, Builli, Buly) Cecilia de, iii, 252 ; Diana, iii, 382 ; Eliz., i, 356,358 ; Eustace de, ii, 336; Hen. de, iii, 219 ; Ingeramus de, iii, I73; Sir Jno. de, iii, I 83 ; Jno., ii, 336 ; iii, 57 . 2 I9, 22 r, 248, 252, 254 ; Matilda, iii, 183 ; Nich. de, iii, 379 ; Pet. de, ii, 294, 336; iii, 252; Sim. de, iii, 2 II ; Thos., ii, 254-5; Will., ii, I 72 ; - , ii, 312,332 ; iii, I3I ; fam., iii, 2 I0

Bow Church (Lond.), iii, 368

Bowdler, Eliz., iii, 286; Thos., iii, 286

Boweles, fam., see Bovil

Bowell, fam., see Bovil

Bowels (Bowells), man., ii, 336 , 337 ; iii, 45, 46, 252, 253

Bowels, fam., see Bovil

Bowels fields (Northill), iii, 245

Bowells Wood (Bromham), iii, 44

Bowen, Alb. E., iii, 88, 92, 95 ; Mrs. iii, 395

Bower, Dr., iii, 297

Bowes Close (Wootton), iii, 332

Bowles, fam., see Bovil

Bowskell, Thos., iii, I7

Bowyer, Sir Will., iii, 393

Box (Boxe), Anne, iii, 220 ; Hen. ii, $28 \mathrm{I} n$; Sir Ralph, iii, 250 Will., ii, 220

Box End (Kempston), mills, iii, 302 Boxes, man., see Throckmortons 


\section{A HISTORY OF BEDFORDSHIRE}

Boxhedge Farm (Cranfield), iii, 275

Box Hill (Surrey), i, 40

Boyden, Mrs., iii, I 46

Boyvill, see Bovil

Bozeat (Northants), iii, 63

Brace, Fran., iii, 154, 240

Bracebridge, John de, iii, 332

Brache, man., ii, $350,356,357,361$, 363 ; mill, ii, 356,366

Brache, Andr. de la, ii, 356, 359

Bracbe Farm (Luton), ii, 375

Brachull, Lescelina de, ii, $381 n$; Will. de, ii, $3^{81} n$

Braci, Aldulf de, iii, 370,372 ; Mascelin de, iii, $37^{\circ}$

Brackley, Thos., Ld., iii, 393

Bradbeer, J., ii, I98

Bradbourne-in-the-Peak, i, 373, 376 ; chaps., i, 372 ; ch., i, 372 ; man, i, $372 n, 377$

Bradenham (Bucks), iii, 4I 5

Bradshaw (Bradshawe), Hen., ii, 361 ; Joan, ii, 36I ; Nich., iii, 221 ; - ii, 216 ; fam., iii, 43

Bradwell (Bucks), i, 6

Brady, Dr., i, 97

Braham, Rich. de, iii, 213

Brahi, see Bray

Braibroc, see Braybrook

Braithwayte, Rich., ii, 245

Brakebergh, see Brogborough

Brakin, Eliz., iii, 200

Bramblehanger, ii, 374 ; man., ii, $350,362,363,366$

Bramblehanger, Alan de, ii, $360 n$, 362

Brambles (Rubi), i, 54-5

Bramingham, Gt. (Luton), ii, 363

Bramingham, Little, ii, 363, 366

Brampton (near Huntingdon), ii,

Brampton, Ld., ii, 235

Bramston, Arth., iii, I 45 ; Withers, iii, I 45 ; Mrs., iii, I 45

Brandreth, Alice, iii, 407; Anne iii, 407; Hen., iii, 391-3, 407 H. C. G., iii, $367,391,393$; Nehemiah, iii, 392 ; Mrs., iii, 391, 393 ; fam., ii, 243 ; iii, $3^{89}$

Branson, G. H., ii, 200

Brantfield, Jno., Ld., ii, 347

Branting. ii, 224

Braose (Brewose), Aliva de, ii, 300, 339; iii, I2, 262; Payn de, ii, 143; Will. de, ii, 300, 339, $34 \mathrm{I}$; iii, $44,219 n, 262,329$

Brasenose Coll. (Oxford), iii, II 7

Brasier, see Brazier

Brasington, Edw., iii, I зо $n$

Brasses, $i, 355 n$; ii, 209, 213, 218 $229,233,236,240,250,258,265$ $269,279,287,292,299,312,319$ $328 n, 331,33^{8}, 343,347,373$ 379 ; iii, $25,28,39,47,53,63,87$ 95, 100, 103, 108, I16, 120, 121, I27, I31 I I35, I 43, I67, 174, I77, I 79, I 85, 193, 200, 208, 2 1 8,222 , $233,235 n, 237,239,240 n, 24 \mathrm{I}$ $250,266,273,283,295,304,307$ $312,315,328,334,340,366,373$, $378,385,387-8,393,4 \mathbf{I}^{\mathrm{I}}, 4^{\mathrm{I}} 3$, $423,425,434,438,446,450$

Brass foundries, ii, 350

Brassington, Edw., ii, 365

Bray, Ada de, ii, 253; Anselm de, ii, 330; Sir Edm., ii, 346; iii, 373 ; Edm., iii, 44, 85, 37r, 397 456; Sir Edw., ii, 340 ; iii, I3. 298, 391 ; Edw., ii, 340 ; iii, $37 \mathrm{I}$, 397 ; Eliz., ii, 340 ; iii, 371 ; Hugh de, ii, 253, 330; iii, 314; Isabella de, iii, I91 ; Jane, iii, 373 ; Joan iii, 97 ; Sir Jno., ii, 340 ; Jno., ii, 301 ; iii, 37r ; Mary, ii, 340 Matilda de, iii, 3 I 4 ; May, Lady,
Bray (cont.)

iii, 298; Sir Reg., ii, 30r, 340 344,346 ; iii, $13,22,44,78,85$, 298, 344, 371, 396; Reg., i, 399 ii, 340 ; iii, 85 ; Rich., ii, 312 iii, 163; Rob. de, ii, 328, 339 : iii, 40, 43, 9r, 98; Rog. de, ii, $34,221,230$; iii, 98 , 191; Temperance, ii, $34 \mathrm{I} n$; Thos. de, $325 n$; ii, $34 n, 330,332$; Will. de, ii, 379 ; iii, 6,427 ; 一, ii, I94 ; fam., iii, 98,2 II, 370, 375

Braybrook (Braibroc, Braybroke), Alianora, iii, r 88; Christina (de), i, 361,364 ; ii, 237, 247 ; iii, I94 Eleanor, iii, $3^{1} 7,380$; Eliz. (de), ii, 296, 324 ; iii, $187,230,317$ 380; Sir Gerard (de), i, 385, 389, $403 n$; ii, 276-7, 280; iii, 52, I30, I $40,162,186,230,243-4$ 380: Gerard de, ii, 35, 276-7, $287 n, 296,323,334$; iii, 65,140 , I 84 , I 86-9, I99, 229-30, 317 333,380 ; Hen. (de), i, 361, 364 $385 n$; ii, $27,28,237,239,247-8$; iii, IO, II, 194, 353; Isabella, ii, $276,280,323$; Joan (de), ii, $276 n$; iii, 186, 199, $230 n$; Sir Jno., iii, 186 ; Jno. de, ii, 72 ; iii, 229 ; Mabel de, iii, 186 ; Marg. de, iii, 187; Maud, iii, 380 ; Sir Reg., ii, 227 ; iii, $230 n$; Reg., ii, 296 ; iii, 140 n, 194 ; Rich. de, iii, 196 ; Rob., i, 329 ; iii, 189, 193-4, 349 ; 一, iii, 140 ; fam., iii, 236 , 326

Braye (Bray), Anne, Lady, ii, 340 ; Jane, Lady, ii, 340 ; iii, 373 : Joan, Lady, iii, 208; Ld., iii, 240, 372-3: Edm., Ld., ii, зог, 340,34 I $n, 343$; iii, 13, 23, 208, 344, 371, 392 ; Jno., Ld., ii, 340 ; iii, $20_{4}, 344$

Brayes, man., see Bromham

Brayfield (Northants), ch., i, $3^{8} 7$, 388,389 ; rectory, i, 389

Brayne Corner (Ridgmont), iii, 32 I Braynforth, Rob., ii, 214

Brays, man. (Husborne Crawley), iii, 396-7

Brazier (Brasier), John, iii, 458; Thos., ii, 310; Will., ii, 312 n

Breach (Maulden), iii, 3I

Breadalbane, Amabel (Amabella), ctss. of, $\mathrm{ii}, 327$

Breauté, Alice de, iii, $70 n$; Falkes (Fawkes, Fulk) de, i, 320, 354, $362,364,378,379$; ii, $27,28,29$, 350 ; iii, 10, II $23,24,282$, 339, 342, 353 ; Marg. de, iii, 11 ; Will. de. iii, ro, II, 70 n ; Will. M. de, ii, 28

Brecknock, Alice, iii, 42y; Dav., iii, 429; Jno., iii, 429; Lettice, iii, 429 ; Marg., iii, 429

Bredham, Jno., iii, I57

Bredyman, Edith, i, $335 n$; iii, 4I9, 436; Edm., iii, 82, 4I9, 436; Geo., ii, 377 ; iii, 82,376 ,

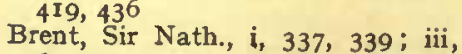
69

Brereton, Rev. C., ii, I74; Jane, Lady, ii, 340 ; Joan, iii, 23,205 , 208, 392 ; Jno., ii, I 73 , 174 ; Thos., ii, I 73 , I74 ; Sir Urian, ii, $34^{\circ}$; iii, 23, 205, 208, 344, 392

Breton, Amabilla le, iii, 405; Gozelin le, iii, 405, 421, 422: Hugb le, iii, 405, 422 ; Juliana le, iii, 422 ; Rich. le, ii, 305 ; Walt. le, iii, 405 ; Will. le, i, 373, 374, 377, 396; see also Briton

Brett (Bretti, Britt), Alice, iii, 428 43I : Hugh, iii, 428, 43I ; Sir
Brett (cont.)

Jno., iii, 259, 260 ; Owen, ii, 44 ; iii, 259, 260; Rob., iii, 443; Will., iii, 443

Bretville, Geoff., ii, 253 ; Will. do, ii, 253

Brewer, Will., ii, 219 ; fam., iii, I94

Brewers' Brash (Wootton), iii, 3II $n$

Brewer's Hill Farm (Houghton Regis), iii, 368,394

Brewose, see Braose

Breybrok, seo Braybrook

Brian, sec Bryan

Briant, see Bryant

Briars Stockings Copse (Eversholt), iii, 375

Bricheno, Will., iii, I 54

Brick Farm (Ravensden), iii, 2 Io

Brickground Plantation, iii, 394

Brickhill, Bow (Bucks), ii, 7I

Brickhill, Gt. (Bucks), i, 376 ; ii, $40,46,53,162$; postal service, ii, 99

Brickhill, Little (Bucks), ii, 2, 3, 46 ; iii, 271

Brick industry, ii, II 7, 261, 304, $3 I_{4}$; iii, I 28, 157, I 75, 186, 2 Io, $215,280,297,307,329,45$

Brickwood, Jno., iii, 194

Brickwork, ii, 364, 365

Brictric, iii, I29, 146

Bridge, Rich., iii, 55

Bridges, Bridg., iii, 255; Brook iii, 21 ; Eliz., iii, 255 ; Giles, iii, 339 ; Haselfoote, iii, 255 ; Thos., iii, 255

Bridgman, Sir Orlando, ii, 52

Bridgewater (Bridgwater), ctsses. of, iii, 41 7, 432; Penelope, ii, 373 ; earls of, iii, $45 \mathrm{I}$; Jno., iii, I $46 \mathrm{n}$, 391, 393 ; Jno. W., iii, 427, 428 ; Arabella, Lady, iii, I37

Bridle, Geo., ii, I72 ; - ii, 173

Bridlington, Geoff., prior of, $i, 353 n$

Brienne, Rob. de, iii,

Briers, Anne, Lady, if, 377-9; Anne, ii, 377: Arabella, Lady, ii, 377 . 379 ; Sir Will., ii, 377-80 ; Will., ii, 379

Brigg (Brigge), Thos. atte, i, $325 n_{i}$ iii, 284 ; fam., iii, 351

Briggs, Geo., iii, 367

Brightman, Thos., ii, 344

Brightwell (Herts), iii, 425

Brihtnoth, ii, 20

Brikulle, Thos. de, iii, 343

Brill (Bucks), i, 400

Brin, Mary, ii, I02

Brinklo, Jno., i, 398

Briscoe, Anne, ii, $295 n$; Jno., ii, 295

Brisey, Mabel, iii, 458 ; Rog., iii, 458

Bristol, Jno., earl of, iii, 45, 49

Bristow, Herb., ii, 256

Britain, Baron, ii, 244; Will., ii, 244

Britens, man., i, 402 ; ii, 82 ; iii, 291, 292

British remains, ii, I, 2, 8, 3 I $_{4}$; iii, I $76,350,402$

Briton (Britton), Hugh, i, 314 n: Jno., ii, 305 ; Marg., ii, 305 ; iii, 292 : Rich., ii, 305: Rog., ii, 305; Will., ii, 305; iii, 292; see also Breton

Britt, see Brett

Brittany, Jno. de, earl of Richmond, see Richmond

Brittons, man., see Britens, man.

Briwere, Joan, iii, II9; Rob. (de la), iii, Irg; Will. (de), iiî, II9, 122, I62 


\section{INDEX}

Broad Green (Cranfield), iii, 275

Broad Green Farm (Cranfield), iii, 278

Broadmead (Wootton), iii, 335

Broc, le, fam., iii, 2 ro

Brocas, Anne, iii, 407; Barn., ii 379 ; iii, 406, 407 ; Edith, iii, 4 I9, 436: Eliz., iii, 407; Jno., iii, 407 ; Rob., ii, 379 ; iii, 406, 407 ; Will., iii, 407

Brocborough, see Brogborough

Brockeborowe, see Brogborough

Brocket, Edw., ii, 357 ; Sir Jno., ii, 355,357 ; J Jo., ii, 357

Brockton, - , ii, 195

Brodo, ii, 232

Brogborough (Lidlington), i, 306

Brogborough (Ridgmont), Cromwell at, iii, $32 \pi$; man., iii, 7 I $n$, 321,322 ; pk., ii, 55, I 47 ; iii, 322 , 323

Brogborough Hill (Kidgmont), iii, 320

Brogborough Pk. Farm (Ridgmont), iii, 320, 321

Brogborough Round House, see Brogborough Pk. Farm

Broi, see Broy

Broilg, see Broy

Broken Wharf (London), iii, 33

Bromeclose (Old Warden), iii, 255

Bromflete, Edw., ii, 277 ; Hen., see Vessy ; Marg., iii, 9I, 118, I2I ; Sir Thos., iii, 91, II8, I19: Thos, iii, 91, I2 I

Bromhall, Oliver, ii, 244

Bromham, ii, 60, 92, 96, I98, 252, 253 ; iii, I $2, I 4,34,37,40,44-9$, 55, 96, 183,302 ; adv., i, $316 n$ $3^{8} 4$; iii, $47,4^{8}$; chant., uii, 49 ; bridge, iii, 36 ; char., iii, 49 ; Chas. I. at, iii, 45 ; ch., i, $315 n$ 382,384 ; iii, $47,48,49,153$. hall, i, 14I; ii, $44,46 n$, 60 ; iii, 44 ; man., iii, $13,37,44,45,46$, $47,78,3$ II ; mills, iii, 44,47 ; pk., $i_{1}$ II2, I20, I26; iii, 44 ; pop., ii, II5: rectory, iii, 47,49 Rom. rem., ii, 5, 6, 15 ; sch., ii, I $8 \mathrm{I}$

Bromham, Jno. de, iii, 47 ; Sim. de, iii, 47

Bromholme, see Bromham

Bromley, Ld. Chancellor, ii, 180

Bromsall, Eliz., iii, 23I ; Frances, iii, 243 ; Marg., iii, 233; Owen T., iii, 231, 244 ; Ralph, ii, 59 ; Thos., ii, 59, 246; iii, 23x, 233 , 243,250

Bronze Age, i, I68-70, 270; iii I I $7,439,44^{8}$

Brooke (Brook), Anne, iii, 4I9: Arth., iii, 419; Duke, iii, 208 209; Eliz., ii, 227: Sir Geo., iii, I 40, $187,208,230,236$; Sir Hen., iii, 208; Joan, ii, 227 ; Marg., iii, 208; Sir Thos., ii, 227 ; Thos., ii, 296; iii, 140, 187, 198; Sir Will., iii, I87, 208 ; fam., ii, 262 ; iii, 205

Brookbanks, A. J., ii, 214

Brook End (Eversholt), iii, 375

Brook End (Keysoe), iii, 136 ; Nonconf., iii, 136

Brook End'(Northill), iii, 242

Brook End (Stotfold), ii, 300

Brook Farm (Holcot), iii, 386

Brook Farm (Ravensden), iii, 209

Brook Farm (Wilden), iii, 223

Brooks, Ann, iii, 286; Cath., iii, 286,288 ; F. G., ii, 199, 200 ; Geo., iii, 6I, 238, 286; Jane, ii, 214,304 ; Jno. H., iii, 286 ; Jno. T., iii, 286 ; Maj. J. H., i, I1 $7,127,137,140$; ii, 146; iii,
Brooks (cont.)

284 i Kath. M. F. B., iii, 284 -, ii, 146

Broom, iii, 227, 256, 260, 26r, 267 hall, iii, 256; Man. Farm., iii, 256

Brotherhood Hall (Leighton Buzzard), iii, 4x 5

Broughberrowe (Lidlington), iii, 305

Broughton (Bucks), iii, I3I, 330, 402 ; adv., i, 383 ; barony of, ii, 294 ; ch., i, 382, 383,384

Broughton, Anne, i, 402 ; iii, 436, 440 ; Cath., iii, 440 ; Eliz., ii, 243; Sir Jno., i, 402; ii, 243 ; Jno. (de), ii, 212, 289; iii, 158 , $346,380,384,436,440,444$; Kath., i, 402; ii, 243, 289; Mary, iii, 346, 440 ; Sir Rob., iii, 436 ; Rob., ii, 243 ; iii, 158,440 ; fam., iii, 312, 381, 446

Broughton End (Lidlington), iii, 305

Broun, see Brown

Brounder, Alice, iii, $x_{40}$; Rich., iii, I 40

Bronnsford, Isabel de, iii, 247 ; Will. de., iii, 247

Brown (Broun), Andr., iii, 433; Eleanor, ii, 257 ; Hen., iii, 35I ; Humph., iii, 23I, 443; Jno., ii, $257,310,342 n$; Rev. J. E., ii, $92 n$; Lancelot (Capability), ii, 325, 355; Rog., i, 326 ; 一, ii, 44, 62, 90 ; see also Brun

Browne, Art., iii, 193; Cath., ii, 347; iii, 443; Christiana, iii, 443; Geo., iii, 443; Lewis, iii, 443; Mabel, Lady, iii, 244 ; Mabel, ii, 323 ; Mary, Lady, ii, 207 ; Mary, ii, 262, $342 n$; iii, 443; Matth., ii, 323 ; Maj.-Gen. Rich., ii, 47,48 ; iii, 402 ; Rich., iii, 244, 250 ; Sir Sam., ii, 206, 207 ; Sam., ii, 44, 55, 204, 262, 265 ; iii, 366; Sir Thos., iii, 244 : Thos., ii, 204, 234, 262, 323; iii, r 33 ; Will., i, 394 ; ii, r58; iii, $32 \mathrm{I}$; see also Brun

Browning, Jno., iii, I 82 ; Capt., ii, r89

Brownlow, earls of, ii, $66 n, 184 n$, $185 n, 220,222$; iii, $368,427,428$, $429,432,448$; Adelbert W., iii, 45I; Jno. W., iii, 45I ; Ld., ii, 216 ; iii, 145

Broy, Margery de, iui, 4o ; Osbert de, iii, 40, 42, 9r : Phil. de, i, $37^{8}$; ii, 26, 27 ; Walt. de, iii, 40 : fam. iii, $43,4^{8}$

Bruce, Ld., ii, 58, 147, 310, 323 ; iii, 286; Ld. Chas., iii, $3 \times 5$; Edw. Ld., ii, 267, 322 ; iii, 267, 294, 337; Ld. Edw., iii, 33I. Rob. Ld., ii, 55, 56, 325 ; iii 290 ; see also Ailesbury, earl of ; Thos., Ld., iii, 290, 31 7

Bruce (Bruse), Alice, iii, 445 ; Chas., iii, 294; Christiana, iii, 299; Gylis, iii, 445 ; Honora, iii, 325 ; Isabel, $\mathrm{ii}, 237,278$; iii, 299 ; Sir Jno., iii, 445; Rich., iii, 299 ; Rob., i, 382, 384 n, 388; ii, $30,237,267,278$ ! iii, 65,195 , 260, 299 : Thos., iii, $318 n$; see also Ailesbury, earl of

Brucebury, man.(Kempston), iii, 299

Brudenell, Alice, iii, I82 ; Cath., ii, 239 ; iii, 150 ; Edm., iii, 150,182 ; Rob., ii, 239 ; iii, 150 ; Sir Thos., iii, $150 n$

Bruert, Will., iii, 189

Brugge, see Brigge

Bruham, see Bromham
Brun (Brune), Jno, le, iii, 129, I30 : Sarah le, iii, I29; Will. le, iii, I29: see also Brown, Browne

Bruneham, see Bromham

Brunstanethorp, ii, 2 I

Bruse, see Bruce

Bruton, Jno., i, 393

Bryan (Brian), Sir Fran., i, 330, $33 x, 367,368 n, 370$; ii, 316 : iii, $197,325,459$ n, $46 \mathrm{r}$; Geoff., ii, 336 ; Iolenta, ii, 336 ; Joan, ii, 2 25, 336; Jno., ii, 325, $345 n$; iii, 274, 296, 316, 418; Mabel, ii, 336; Pet., ii, 336; iii, 418: Rob,, ii, 336 ; iii, 4I8 ; Rog., ii, 215,216 ; Sir Will., iii, 382

Bryant (Briant), Jno., iii, 26r, 393, 414 ; J., ii, 292 ; iii, 374

Bryteville, Hugh de, iii, 182; Jno. de, iii, r82; Will. de, iii, 182 Brytvilles, man., see Birchfield

Buchanan, Arch., ii, $3^{85}$; Jane, ii, 385

Buck, Sir Chas., iii, 436, $43^{8}$; Frances, iii, 436 ; Sir John, iii, 436; Mary, Lady, iii, 436; Sir Will., iii, 436

Buckby (Northants), i, 360

Buckby, Anne, ii, 281 $n$; Mary, ii, 281 ; Rich., ii, 281, 285; Will., ii, 265,281

Buckden (Hunts), ii, 38, 39

Buckhurst, Ld., ii, 253

Buckingham, ii, 47, 67; iii, I ch., i, 3II

Buckingham, Anne, duchess of, iii, 173 ; dks. of, ii, 53 ; iii, Iro, 154 , 298; Edw., iii, 106, 126, 156, 172 : Hen., iii, x 71, 283; Humph. iii, 78,85 , I 7 I-2, 422 ; Hen., earl of, iii, 36; Humph., earl of, iii, 36 ; Walt., earl of, ii, 385 ; iii, 315

Buckingham, Jno., ses Lincoln, bps. of ; Ricli. of, $i, 397$; Will. of, i, 397

Buckinghamshire, archd. of, i $354 n$; iii, 28 ; bishopric, i, 332 . ind., ii, I2I, 123, 124 ; parl. repre., ii, 34 ; sheriffs of, ii, $29 n, 30 n$, $32,4^{\circ}$

Buckland, Joan de, iii, 452 ; Matilda de, iii, 452 ; Will. de., iii, 452

Bucklow, iii, $34-5,36 n$

Buckmaster, C., iii, 4I 7 ; Jno., iii, 415, 449 ; Rich., iii, 415

Buckstead (Studham), iii, 368

Buckwood Stubbs, iii, 337, 390

Buckworth, Jos. F., iii, 330 ; Mary, iii, 330

Buddenho (Berells and Frenches), man., iii, $247^{-8}$

Buddenho (Bodenho), Jno. de, iii, 30 ; Nicolaa de, iii, 247 ; Rich. de, iii, 247

Budewell (Turvey), iii, $x_{4}$

Budna, iii, 242, 248, 250

Bueles (Buels), fam., see Bovil

Bugge, Reg., iii, 43

Builli, fam., see Bovil

Bulkeley (Bulkley), Ann, lii, 212 : Edw., iii, 69; Joan, iii, 24I ; Jno., iii, 212 ; Pet., i, 337 ; iii, 69 ; Rob., iii, 212, 24I ; Rog., iii, 24I ; Will., iii, 212 ; see also Burdelys

Bulkeleys, man., see Burdelys

Bulkley, fam., see Bulkeley

Bull, Jas., ii, ror

Bulle, Thos., ii, $15 x$

Bullen, Rev. Hen. St. J., ii, 259 Sarah, ii, 259

Buller, Mary, iii, 332

Bullisdon, Thos., iii, 250

Bullok (Bullock), Eliz., ii, 224 ; Jno., ii, 224; iii, 9r ; Kath., ii, 224 ; Rob., ii, 224 


\section{A HISTORY OF BEDFORDSHIRE}

Bulstrode, Edm. (de), i, $326 n$; iii, $436 n$; Rich., iii, $278 n$

Bultban, Judith, ii, 289

Buly, fam., see Bovil

Bunbury, Sir Chas., ii, 192, 193

Buncer's Farm, see Caddington

Bunion (Buniun), fam., see Bunyan

Bunker, Rich., iii, 375

Bunting, Rev. Edw. S., iii, I 79

Buntingford (Herts), ii, 359

Bunyan, Eliz., iii, 207 ; Hen., ii, $376,377 n, 380$; Jno., i, I03, $340-5$; ii, $47,49,59-60,374$, $377 n$; iii, $5,7,8,22,32,179$ $207,279,282,290,316,379,45 \mathrm{I}$; Thos., iii, 279, 290; Will., iii, 279 ; fam., ii, 376,377

Bunyan Institute, Bedford, iii, 32

Burcester (Oxon), man., i, 360

Burdelys, man. (Gt. Barford); iii, 182-3

Burdelys, man. (Stagsden), iii, 97

Burdelys, Eliz. de, iii, 98 ; Geoff. de, iii $98,98 n, 182$; Hugh de, iii, 182; Joan de, iii, 98; Jno. de, iii, $97,98,182$; Will. de, iii, 182 ; sce also Bulkeley

Burdett (Burdet), Mich., ii, 227 : Nich., ii, 238; Will., ii, 238

Burdon (Cambs), i, 364 ; iii, 247

Burdun, Walt., iii, 129

Bures (Essex), Rom. rem., ii, I3

Bures, Andr. de, iii, 453 ; Kath. de, iii, 453

Burford, barons of, iii, 317

Burgam, Sir Rog, ii, Igr

Burgend Close (Bedford), iii, 32

Burgess, Jno., iii, 442; Thos., iii, $44^{2}$

Burgh, Beatrix de, iii, 339 ; Hawise de, iii, 339 ; Hubert de, i, 319 ; ii, $27-9$; iii, $70,339,340,342$ : see also Kient, earl of; Isabel de, iii, 70 ; Jno. de, iii, 339; Marg. de, ii, 339: Matilda de, see Ulster, ctss. of ; Ralph, ii, 374; Rob. de, iii, 276

Burghersh, Sir Barth. (de), iii, I29, 426 ; Barth. (de), iii, I29, 426 Hen. de, iii, 426-7; Maud, iii, 429; -, bp., see Lincoln, bps.

Burghley, Dorothy, Lady, see Exeter, ctss. of; Ld., iii, I 3, 459: Thos. Ld., see Exeter, earl of

Burghope, Rev. Geo., iii, 432

Burgoyne (Burgoine), Constance, ii, 241, 250; Fliz., ii, 250 ; Sir Jno., ii, 42, 44, 45, $66 n, 68 n, 70$, I97, 248, 250; iii, 20I, 20I $n$. Jno., ii, 39, 40, 220, 222, 228 , $238,239,244,245,248,250$; iii, 329 ; Sir Jno. M., ii, $66 n$, 246; Marg., iii, 329: Montague, i, 250; Sir Montague R., ii, 256 ; Rich., ii, 238, 239 ; iii, 329 ; Rob., ii, 244, 347; Sir Rog., ii 44, 62, 239, 248, 250 ; Thos., ii, 220, 238, 239, 248, 250 ; Thos. J., ii, 24I ; Will., iii, 21, 329 ; fam. ii, $44,66,249$

Burials and burial urns, $i, x_{59}, 160$, $163, \mathrm{x} 68,183,184,185,187-89$ $270,296,297,350$; ii, I-I4; iii, $402,44^{8}$

Burke, Edm., iii, 54

Burlee, Will., iii, 259

Burley Farm (Stevington), iii, Io2 Burnaby, Jno. de, iii, I29; Rev. J. B., iii, 323

Burnard, Felicia, ii, 227 ; Hen., ii, 263 ; Nich., ii, 227 ; Odo, ii, 227 , 262 ; Rog., ii, 226, 262 ; Steph. ii, 262 ; - ii, 261, 263; see also Barnard and Bernard
Burne, Jno., i, 380 ; iii, 22

Burneham Mead, ii, 266

Burnel (Burnell), Phil., iii, II8, Ix9 n, I22, I59; Sir Rob., iii, 122 ; Rob., iii, I1 8 ; fam., iii, 283 Burnet, Phil., iii, 55

Burr (Burre), Agnes, iii, 444 ; Rob., i, 387 ; Will., iii, 444

Burret, iii, I39, I 42

Burreth, Thos., i, 402

Burrland, Rich., iii, 374

Burroughs, Rev. Lynch, ii, 310; Sarah, ii, 310

Burr's Estate (Dunstable), iii, 368

Burt, Thos., iii, 64

Burton, Alex. J., iii, I 45 ; Marg., i, 393; Mrs., iii, I 45

Bury, Frances, iii, 340 ; Fran., iii, 340 ; Geo., iii, 278,286 ; Hadwisa, iii, I90, I92; Jno. de, iii, 190, I92 ; Rich., iii, 407, 443

Bury End (Shillington), ii, 294; chap., ii, 299

Bury End (Stagsden), iii, 96

Bury Farm (Goldington), iii, 202

Bury Farm (Houghton Conquest), iii, 289

Buryfield (Houghton Regis), Rom. pottery, ii, 8

Bury Hatley, man., see Cockayne Hatley

Bury Hill (Thurleigh), earthworks, i, 287

Bury St. Edmunds (Suffolk), i, 328 ; abbot of, iii, 228, 229

Buryssteede (Salphobury), man., iii, 2 I $5 n$

Busard (Buzzard), fam., iii, 40

Busby, Will., iii, I62

Bush, Eleanor, ii, 362 ; Ralph, ii, 362

Bushey (Herts), i, 36o

Bushmead Priory, i, 349, 350, 385-7; ii, I49, 150; iii, 24, 137 , 166, 180, 184, 187, 188, 189, 190, 193, 194, 197-9, 225, 292 ; ch., i, 386; earthworks, i, 307 ; man., iii, 198; priors, i, 328, 385-7; ii, 23 ; iii, $137,166,194$; sch., ii, 149 Bushmead Wood (Eaton Socon), iii, I 89

Bushycommon Wood (Potsgrove), iii, 421

Bussey (Bussy), Andr., iii, 237 ; Barth. de, i, $362 n$; ii, $230 n$; Cecily (Cecilia) de, i, 36I $n$; ii, 230 ; iii, 255 ; Cecill, iii, 237 ; Eliz., iii, 237 ; Hadwisa de, ii, 230 ; iii, 252 ; Joan, ii, 232 ; Jno., iii, 237; Maud de, i, 36I $n$; ii, 23o, 231, 233; iii, 255; Will. de, i, $36 \mathrm{I} n, 362 n$; ii, 230,233 ; iii, 252, 255 ; fam., iii, 237

Busshey Close (Renbold), iii, 2 I 5

Bussy, fam., see Bussey

Butcher, Frances E., iii, 2 Io Rich., ii, $x 62$; Rev. Rob. H. iii, 2 IO

Bute, earls of, ii, $6 \mathrm{r}, 307,353,355$; mqs. of, i, 330 ; ii, 6I $n, 349 n$, $353,356,358,366,374$ : iii, 257

Bute Hosp. (Luton), ii, 375

Butler, Ld. Will., ii, 347 ; Anne, Lady, lii, I05; Anne, ii, 347 ; Audrey, ii, 347 ; Beckingham, ii, 206, 301 ; Sir Edw., ii, 345, 38I ; Edw., ii, 206, 215, 301, 302, 345 ; iii, I 26, 385 ; Eliz., ii, 345 ; Florence, ii, 289: Geo., ii, 40, 30r : Hawise (Hadwisa), ii, 345, 38r ; Helen, ii, 347; Hen., ii, 347 ; Jane, ii, 345,347 ; Joan, ii, 2 I 5,289 ; Sir Jno., ii, 345,347 ; Ino., ii, 165, I66, 289, 290, $292,345,347,359,382$; iii,
Butler, (cont.)

46; Kath., ii, 292; Mary, ii, 347; Nich., ii, 345 ; Olive, ii, 347 ; Sir Oliver, iii, 105, 33I Oliver, iii, 21 ; Sir Phil., ii, 345, 381,382 ; iii, 105; Phil., ii, 345 Ralph, ii, $345,346,38 \mathrm{I}$; iii, 46 Sir Rob., ii, 345; Rob., ii, $3^{82}$ Sam., iii, 238 ; Thos., ii, 165,345 , 359 ; iii, 244, 278, 432 ; Sir Will., iii, 21 ; Will., ii, $59,345,359$, $3^{82}$; iii, 38 ; Maj., ii, 54, 55 fam., ii, 330 ; see also Boteler

Butler's Farm (Northill), see Cook's Farm

Butt, Sir Will., iii, I 34

Butt Close Park, iii, 394

Butter, Will., iii, $374 n$

Butterfield, W. R., i, I Ix, II

Butterflies, see Rhopalocera

Butterworth, Mrs., iii, I22

Button, Ricb., iii, 331, 332

Butts, Little (Dunstable), ii, 7

Butvillein, Alice, iii, $84 n$; Eliz., iii, $84 n$; Joan, iii, 84 ; Juliana, iii, 58,84 ; Nichola, iii, 84 ; Rob. iii, 84 ; Will., iii, 58,84

Buzum, fam., see Bosom

Bycham, see Beauchamp

Byddik, Ant., ii, 321; Christina, ii, $32 x$; Jno. de, ii, 322

Bygod, see Bigod

Bykeleswade, see Biggleswade

Bylindon, see Billington

Byng, Sir Geo., ii, 6o, 6I, $64 n$; iii, $246,253,258,26 \mathrm{I}$; see also Torrington, vsct. ; Adm. Jno., ii, $6 \mathrm{x}$; iii, 260 ; Jno., iii, 246 ; Pattee, ii, 62, 29I ; Sarah, ii, 6o

Byng of Southill, baron, iii, 258

Geo., baron, ii, 6I

Bynnion, Sir Geo., ii, 43

Byrche, Hen., ii, $3^{87}$; Will., ii, $3^{8}$

Byrling, Alex., iii, 293; Alice, iii, 293 ; Jno., iii, 293

Byscote, see Biscott

Byssopesdon, Will. de, iii, 23 I $n$

Bytham Castle (Lincs), iii, $\mathrm{I}_{44}$

Cabbin Ground (Bromham), iii, 44

Cadbury, man., iii, 193, I94, 197 $198 n$

Caddington, i, 258, 338; ii, 71,89 $306,314,316,320$; iii, 367 adv., ii, 319, 320 ; Buncer's Farm, ii, 314; Bury Farm, ii, 315 char., ii, 320 ; ch., i, I5I, 3I 2 ii, $6,314,318,319,320$ : - Five Horse Shoes Inn, ii, 318 ; man. i, 312 ; ii. $3 x_{4}$; mill, ii, 315 Nonconf., ii, 320 ; iii, 367 ; placenames, ii, 314; pop., ii, Ix4 prebends of, $i, 3$ I2, 324; prehist. rem., $i, x_{45}-6, x_{5 x}, x_{54-8}$ I63, I66, I72, I74; ii, $3 I_{4}$ Rom. rem., ii, 6 ; sch., ii, $18 \mathrm{x}$. vicarage, $i, 317$

Caddington, Gt., man., ii, $3^{\text {I }} 5$ prebend, ii, 3I5: Prebendal Farm, ii, 3i 5

Caddington, Little, man., ii, 315 ; vicarage, ii, 3I 5

Caddington, Rich. de, ii, 309, 310 Sibyl de, ii, 309, 310; Walt. of, i, 384

Caddington Bury, ii, 315

Caddington Green, sch., ii, 18

Caddington Hall, ii, 316, 318

Caddington Hills, i, 15

Caddington Minor, prebend of, ii 3 I 5

Cade, Jack, rebellion of, iii, 359

Cadendone, see Caddington 


\section{INDEX}

Cadenton, see Caddington

Cadwell (Holwell), ii, 286

Cadyndone, see Caddington

Cacn, Paul of, see St. Albans, abbot of

Caesar, Sir Julius, ii, I 59

Caesar's Camp (Sandy), A.S. rem., i, 184, 242; Rom. rem;, i, 271, $272,273,274$; ii, 9, 10

Cainhoe, ii, 30,76 ; chap., i, 318 , $35 \mathrm{I} n, 352$; ii, $324-5$; land value of, ii, 91 ; man., ii, $80,322,323$, 325 ; iii, 273 ; mills, ii, 323,324 ; place-names, ii, 321

Cainhoe, bar, ii, $277,329,376,377$, $379,382 n, 383$; iii, 50, 5I, 54, 60, г г $3,1_{4} 6,236,237,286,309$, $346,379,396,435-6$

Cainhoe, hon., ii, $32 \mathrm{I}$

Cainhoe, Lady Isabel of, ii, 32

Cainhoe, Pet., iii, $27^{8}$

Cainhoe Castle (Clophill), i, 29r-2, 297 ; ii, 32 I, 324

Cainboe Farm (Clophill), ii, 32 I

Caisneto, see Cheney

Caisot, Caissot, see Keysoe

Caius Coll. (Camb.), i, 342 ; ii, 162, I6 5 ; iii, $\mathbf{r} 79$

Calcutt Farm (Houghton Regis), see Carcutt

Caldecote, man. (Herts), i, 386

Caldecote, man. (Houghton Regis), iii, 392

Caldecote, Lower, iii, 242, 246, 25 I

Caldecote, Upper, iii, 242, 246 ; chap., iii, 250; ch., iii, 242 : man., iii, 246, 247 ; Rom. rem., ii, 9

Caldecote (Caldecot), Amice de, iii, I38; John, iii, I25

Caldecote Lodge (Northill), iii, 242

Caldwell, i, 3I $5 n, 3$ I6 $n$; man., iii, 2 I

Caldwell, John of, i, 353

Caldwell Priory, $i, 317 n, 322,325$, $328,349,374,379,382-7,398 n$; ii, $244^{-5}$; iii, II, 2 I, $38,47,48$, $92,107,147,221,225,286,302$, 333,377 ; seal, $i, 3^{8} 5$

Calfe, Susannah, iii, 408

Calhoun, Will., ii, 64, 65 : iii, 20

California (Dunstable), i, r6o

Callopp, Reg., ii, 379; Thos., ii, 379

Calverly (Calvely), Agnes, Lady, ii, 234; iii, 72, 384; Sir Geo., ii, 234 ; iii, 72, 384 ; Mary de, iii, 220 ; Oliver (de), iii, 219, 220

Calvinist Baptists, see Baptists

Cam, riv, ii, 7 I

Cambleton, see Campton

Cambridge, ii, $20,25 n, 48,50,5$ I, 79, I38, I99; iii, I, 10

Cambridge, Maud, ctss. of, iii, I3 ; earl of, iii, I3

Cambridgeshire, ii, I9, 25, 7I, 9I, II 7

Camden, Lady, ii, I8o, 235

Camelton, see Campton

Camera, Rob. de, iii, I 47

Camestone, see Kempston

Camoys, man., see Honydon

Camoys, Hugh, iii, r94; Joan, iii, 84: John, iii, 194; Marg., iii, I94; Sir Ralph, iii, 84 ; Rich., iii, 194 ; Sir Thos., iii, I94

Campbell, Amabel, ii, 327 ; Jemima, ii, 327 ; iii, 65 ; Sir John, iii, 19 ; Rob., iii, 293

Campden (Gloucs.), ii, 224

Campion, Andr., iii, 392 ; Maj. Coventry, iii, 45I, 453; Edw. iii, 51 ; Sir Hen., ii, 42 ; Hen., iii, 42 ; Rev. J. W. C., iii, 453 ; Mrs., iii, $453 ;-$, iii, 125
Campion's Hill (Wootton), iii, 33I Campton, ii, 260, 266-76: iii, 77 , 259, 278; adv, ii, 270; Bury Farm, ii, 270 ; char., ii, 270 ; ch., i, $315 n$; ii, 266, 269-70 ; common, ii, 97 ; Grange, ii, 266 ; Highland Farm, ii, 266 ; mans., ii, 267, 268, $27 \mathrm{I}$; man.-bouse, ii, 268 ; mkts. and fairs, ii, 268 ; mills, ii, 266 , 268-9 ; Nonconf., ii, 270 ; pop., ii, I13 ; rectory, ii, 265; R.C., ii, 266 ; sch., ii, I8I, 266

Campton-cum-Shefford, see Campton

Campton House, ii, 266

Canal Close (Ampthill), iii, 274

Canals, ii, 108; iii, 363,400

Cane, see Kane

Cannon, Tom, ii, I94

Canons (Cannons), man., iii, 329, $33 \mathrm{I}-2$

Canons Ashby Priory, ii, 326 ; iii, $85,87, \mathrm{IO}_{4}, \mathrm{I08}, \mathrm{I09}, 126$

Canons (Cannons) Hill (Wootton), iii, 33I $n$

Cantelow(e), see Cantilupe

Canterbury, A.S. rem., i, I84 ; sch., ii, 152

Canterbury, archbps. of, i, I9o, 322, $333,346,353 n, 357 n, 372,374$, $375,389 n, 399,399 n, 400$; ii, $35,1_{54}, 1_{59}, 203,2$ 10, 219, 293, $312,335,359$; iii, I4, 46, 70, 72, $89,133,140,182,260,270 n$, $272 n, 293,317,372,403 n$

Cantia, Cecily de, $i, 390$; Laura de, i, 360 ; see also Kancia

Cantilupe (Cantlow, Cantelow), Agnes de, ii, 251 ; Geo. (de), ii, 32 ; iii, I I $8,370,44^{8}$; Hen., iii, 456 ; Joan de, iii, 229, 370 ; John, iii, 456 ; Mascelin de, iii, 370 ; Millicent (de), ii, 225, 282; iii, I18, $37^{\circ}$; Rich., iii, 456; Rog. de, ii, 251 , 253: Thos, ii, 32; Walt, see Worcester, bp. of; Will. (de), i, $320,325 n$; ii, $27,32,225,282$; iii, II $8,359,370,372,374,39$ r, $430,44^{8}, 455-6$; fam., ii, 28, 224

Cantlow, see Cantilupe

Capel, Ld., ii, 43, 45, 53, 54

Capel (Capell), Sir Arth., iii, 254 ; Eliz., iii, 254, 427

Capella, Hen., iii, 339

Caperon, Agnes, iii, 74 ; Ralph, iii, 74 ; Sybil, iii, 74

Capon, John, iii, 407

Carcutt Calcutt Farm (Houghton Regis), iii, 390, 392

Cardington, ii, 21, 44, 66, 92, 255 ; iii, $3 \mathbf{r}, 33,45,166,197 n, 216$, 233, 28I, 292; adv., iii, 237 agriculture, ii, r34; bridge, iii, $235 n$; char., iii, 238 ; ch., i, $315 n, 380$; iii, 233, $235 n$, 236-7: cross, iii, 234 ; man., i 381 ; ii, 299 ; iii, I3, I5, 234-5 man. farm, iii, 233; man.-house, i, 306 ; mills, iii, 234-5; Nonconf. iii, 234; pop., ii, II6; prebist. rem., i, I 45, I 74 ; iii, 234 ; sch. ii, 181 ; vicarage, $i, 316 n$

Cardington Brook, iii, 233, 235 n

Cardington Cotton End, man., iii, 236

Cardington Priors, man., see Fenlake Barns

Cardington with Eastcotts, iii, 233-8

Cardun, Isabella (de), iii, I19, 122

Cardyngton, see Cardington

Carew (Carewe), Eleanor, Lady, ii, 355 ; Eliz., iii, 424; Jas., iii, 427 ; Marg., iii, 427 ; Nich., iii, 427 : Sir Rich., ii, 355
Carewrike, Will., iii, 306

Carey, Adolphus, iii, 405 : Anne, iii, 405; Eliz., ii, 220: Hen., see Hunsdon, Ld. ; Walt., ii, 228, 229

Carldon, see Carlton

Carlentone, see Carlton

Carleton, see Carlton

Carlisle, Honoria, ctss. of, iii, 330 Jas., earl of, iii, 330

Carlisle (Carlyll), Alice, iii, 4I I Joan, ii, 269; Nich., ii, I6I Rich., ii, 269 ; iii, 22 I

Carlton, ii, Ir $5, \mathrm{I}_{45}$; iii, $34,49,50$ 5I, 76, 77, IOo, I I 4 ; adv., iii, 5I, $54-5$; ch., ii, 92 ; iii, $49,52-4$ 56 ; man., iii, 49-5I, 54-5, $224 n$; place-names, iii, 49 ; rectory, iii, 56 ; sch., ii, I $8 \mathrm{I}$

Carlton (Carldon, Carleton, Karle ton), Geoff., iii, 52 ; Geo., iii, 304 ; Godf. de, iii, 55; Rich., ii, 230 : iii, 443 ; see also Charleton

Carlton Hall, man., iii, 5I-2

Carlyles, man. (Roxton), iii, 22

Carlyll, see Carlisle

Carmino, Joan de, iii, 246 ; John de iii, 246; Ralph de, iii, 246 . Walt. de, iii, 246

Carminos, see Caldecote, Lower

Carnarvon, Anna S., ctss. of, iii, 427 ; Eliz., ctss. of, iii, 427 earls of, ii, 5o; Chas., iii, 427 ; Rob., iii, 427

Carnivora, i, 139

Caron, see Carun

Carpenter, Edwin O., ii, I9o ; John i, 402 ; Mrs., iii, 297

Carr, Will., ii, 180

Cart, Jane, ii, 179 ; iii, $351,367-8$

Carter, Anne, iii, 305; Edw., iii 191; Geo., ii, 188; Howard, ii, 263 ; H., ii, 26I; Ralph le, iii, 333 ; Thos., iii, II 7, 302 ; Will. (le), iii, 274, 302, 304, 333

Carteret, Lds., ii, I33, I46, 210 iii, 328; Geo., iii, 29 ; Hen., iii 326 ; Hen. F., ii, 6o, 34I, 343 ; John, ii, 6o, 341 ; iii, 132,326

Carteret, Sir Geo., ii, 6o, 339, 34 I iii, I4 ; Jemima, Lady, ii, 34 I $n$ Sir Phil., ii, 34I

Cartwick, Will., ii, 322

Cartwright, Edm., ii, $281 n$; Hugh, iii, 258-9 ; Rich., ii, 255 ; iii, 393 Will., iii, 258-9; Dr., ii, 135

Carun (Caron, Karun), Agnes de, ii, 25I ; Amice (Amicia) de, ii, $253,276 n$; Eliz. de, ii, 276 Flandrina, iii, 66; Hugh de, ii, 276: Joanna de, ii, 25I; John de, ii, 25I, 276 : Lucy de, ii, 276 ; Margery de, ii, 276; Miles de, ii, $276 n$; Osbert de, iii, $196 n$; Ralph de, iii, 66 ; Rob. de, ii, 25I, 253; iii, 76 ; Walt. de, ii, $25 \mathbf{I}$, 252,253 ; iii, r96; Will. de (le), ii $25 \mathrm{I}, 252,253,275 n, 276-8$. iii, 259-60

Caryer, Jobn, ii, 24

Cason, Edw., iii, 3 I $_{4} n$; Jane, ii 347; John, iii, 236; Julian, ii, 347

Castelacre, Will. de, iii, 194

Castell, Edw., ii, 167; Mary, iii, 205 ; Rob., iii, 205 ; Dr., ii, 347

Castle Ashby, ii, 4 r ; iii, 293

Castle Close (Goldington), iii, 206

Castle Hill (Bedford), iii, 33

Castle Hill (Eaton Socon), iii, I 89

Castle House (Berkhampstead), $\cdot$ ii, 317

Castle Mill (Goldington), iii, 206

Castor (Northants), iii, 300

Castre, Will. de, iii, 168 


\section{A HISTORY OF BEDFORDSHIRE}

Catelayne, Lady, i, $335 n$; see also Catlin

Cater, Anne, iii, 3or ; Beckford K., iii, 301 : Edw., iii, 3or ; Frances K., iii, 301; John, iii, 301 ; John K., iii, 30I ; Marg. K., iii, 301 ; Mary, iii, zor ; May K., iii, 30I ; Sir Rob. K., iii, zor : Sam., iii, 3or ; Sophia K., iii, 3or ; Will., iii, 300,305 ; fam., iii, 303: see also Cator

Catesby, man., i, 376

Catesby (Catesbie), Ant., ii, 359 iii, $177 n$, 310; Bridg., iii, 310; Clifton, iii, 3ro: Dorothy, iii, I $77 n$; Geo., ii, 359 ; iii, 310; Humph., ii, 359: iii, 310; Sir John, iii, 310: John, ii, 40, 359 ; Thos., ii, 359, 363, 365 ; iii, 3 Io Will., iii, I 73

Cates Close (Wootton), iii, $331 n$

Cateswood (Eaton Socon), iii, r89, I94

Catherine (of Aragon), (queen), i, 374,376 ; ii, 38, 122, 123; i iii,

Catherine (of Braganza), (queen), iii, 227

Catherine, St., ii, $38 n$, 122, I 23

Catlin, Eliz., ii, 257; John, ii, 257 Mary, ii, 232, 243: Sir Rob. ii, 232, 243 ; see also Catelayne

Caton, Dan., ii, 28 I

Cator, Edw., ii, $54 n, 55$; - , ii, 44 ; see also Cater

Catuvellanni, ii,

Catworth, Gt. (Hunts), iii, 136

Catyeuchlani, see Catuvellauni

Cauceys, Geoff. de, i, $372 n$

Cauf, Will. le, iii, 444

Caulcott (Marston Moretaine), iii, 307 ; brick kilns, iii, 308 ; char., iii, $3^{1} 3$

Cauldwell, crown property in, iii, 271 ; price of land, ii, 91

Cauntelow, see Cantilupe

Caurel, Pierre, ii, 358

Causeway End (Wilshamstead), iii, 328

Causum, see Caversham

Cauvin, - (of Bedford), iii, ro2

Cauz (Caus), Jas. de, i, $326 n$; ii, $3^{82}, 3^{8} 4$; Nichola, iii, I0 4 ; Rich. de, ii, $3^{82}$; Rog. de, iii, $\mathrm{IO}_{4}$

Cavendish, Alice, ii, 334; iii, 429 ; Chas., ii, $45 n$; Frances, iii, 43 ; Geo., iii, 429; John, ii, 334 ; Thos., iii, 429 ; Will., iii, 43

Caversham (Causum), ii, 51

Cawne, -, iii, r9-20

Cawse, R. J., i, I34

Cealhgnefaw, see Chalgrave

Cecil, Dav., see Exeter, earl of : Lady Diana, iii, 315; Dorothy, Lady, see Exeter, ctss, of ; Hen., see Exeter, marquess of ; Mary, iii, 330: Sir Thos,, see Exeter, earl of ; Thos., iii, 285, 288; Will., iii, I 4

Celestine III, pope, iii, Ir6

Celgrave, see Chalgrave

Cella, John de, ii, 36 I

Cell Dene (Caddington), ii, 318

Cement and lime works, ii, II 7 , $26 \mathrm{I}, 384$; iii, 447

Cemeteries, A.S., i, I 75-90 ; iii, 402 ; Bronze Age, iii, 448; Rom., i 18 7; ii, 5, 9, II : Rom. Brit., ii,

Cerlentone, see Charlton

Cernes, Nich. de, ii, 252, 253 ; Thos. de, ii, 253

Chad, -, iii, 415

Chadwell End (Pertenhall), iii, 153
Chainhalle, see Channels End

Chaisot, see Keysoe

Chalenor, Rog., iii, 300; Thos., iii, 300

Chalfont St. Peter (Bucks), ii, I 20

Chalgrave, i, 376,402 ; ii, 43,318 ,

353,375 ; iii, $89,336,344,383$;

adv., iii, $346,348,349$; chant., i, $325 n$, 329; iii, 349 ; char., ii, I8r $n$; iii, 349 ; ch., i, 3 II $n$, 3I $5 n, 3$ I $8,325 n, 37$ I $n$; iii, 345 , $347,348,349$ : ind., iii, 345 : man., ii, 22 ; iii, $345,346,347$, $380 n$; Manor Farm, iii, 345 Nonconf., iii, 345 : 'Old Orchard,' iii, 349; place-names, iii, 345 ; pop., ij, II 4 : rectory, iii, 346 , 348,349 ; schs., ii, I 81 ; iii, 345 , 349 ; Shoulder of Mutton Inn, iii, 345 ; vicarage, $\mathrm{i}, 3$ I $6 n$; iii, $345,34^{8}$

Chalgrave Hockliffe, see Hockliffe

Chalk, - iii, 226; fam., iii, 224

Chalk Hill, see Puddle Hill

Challeye, Sim., ii, 89

Chalmers, - iii, I 25

Chalton, iii, 227, 228, 230, 23r, 362, $394,439,440,442$; chap., iii, $446 n$; man., iii, 23I, 442, 443, 444 Chalton, Ann, iii, 175

Chalton Cross Farm (Toddington), iii, 439

Chalverston, i, 384

Cham, John, iii, 187 ; Odo le, iii, 187; Reg. le, iii, 187 ; Rich. iii, 187 ; Rob. le, iii, 187 Sabina le, iii, 187 ; Sim. iii, 187

Chamber, Edw. atte, iii, 182; Gilb. atte, iii, 98 ; Joan atte, iii, 98

Chamberlain (Chamberlin), Amabel, iii, 152 ; Amphyllis, iii, 315 ; Aubrey, iii, 406; Dray, iii, 277 ; Edw., iii, 433; Eliz., iii, 433; Geoff., ii, 2r9, 22r ; Hugh, ii, 219; Joan, iii, I 50, 406 ; John, iii, II2, 406; Sir Leonard, iii, 361 ; Marg., iii, 406, 433; Sir Rich., iii, I52, 406, 433; Rich. iii, 225, 406, 433 ; Rob., ii, 219 ; Sim., iii, $446 n$; Sybil, iii, 433 . Thos., iii, 406; Turstin, iii, 77 ; Will. (the), ii, $22 x, 356,373$. iii, 393, 423, 433, 449; fam., iii, 409

Chamberlains Bury, man., ii, 220

Chambers, -, ii, 162

Champion, Rich., iii, 286, 307, 333

Chancellor, Thos. the, $i, 366 n$

Chancellor, the Ld., ii, I 70, I 73, 175 Chandler, Anne, iii, 183 ; Ant., iii, 393, 454; Dav., iii, I83; Greo. iii, 374,414 ; John, iii, 152, I 56 ; Rich., ii, 209, 254, 269, 332, 335 : iii, 29, 208, 250, 268, 319, 388 , 414, 420; -, iii, 226, 43I, 446, 450

Chandos, Cassandra, dchss. of, iii, 255 ; Jas., dk. of, iii, 255

Chanewe, Will., i, $3^{87}$

Channels End (Colmworth), iii, 18o, 186

Chantrey, Sir Fran., iii, $4^{18}$

Chantrics, i, 325, 326, 329, 383 , 397 ; ii, $155,156,242,299,318$ $325,326,330,332,333,335,368$, $369,374,381,382,384$; iii, 30 , $40,43,49,92 n, 94$, I 18 , I 26, I36, I 53, 201, 209, 21 6, 233, 244 $249,250,251,255,266,274$ 28I $n, 284,304,305,328,335$, $342,349,3^{6} 7,374,375,382,386$ $39 \mathrm{I}, 394,4 \mathrm{I} 5,421,423,428,432$ $438,446,453,454,455$; foundation, i, 325 ; suppression, i, 3.3 ; ii, 151,155
Chantry Farnı (Sharpenhoe), ii, $313,3^{8} 4$

Chapel End (Cardington), iii, 234

Chapel End (Colmworth), iii, 186

Chapel End (Houghton Conquest), iji, 289

Chapel End (Wilshamstead), iii, 326

Chapel Herne, iii, 21, 22

Chapel Hill (Hinwick), iii, 8I

Chapel of Saint Macutes (St. Machutus, St. Makes, St. Marks), man., ii, 342

Chapels, anct., ii, 269, 320, 343, 365 , $368,369,370,372,373$; iii, 49 , $255,283,304,313,349,365,374$, $430,443,446,459,46 \mathrm{I}$

Chapman, Rebecca, iii, $33^{\circ}$; Shuckburgh, iii, $72 n$; Thos., iii, $33^{\circ}$;

Walt. le, iii, $358 n$

Chapman \& Mears, ii, 245, 319

Chappell's Groundes (Leighton Buzzard), iii, 404

Charles I, ii, 4I, 47, 50-3, 56, I6I, 201, 210, 26r, 327, 353 ; iii, $4 \mathrm{r}$, $45,123,172,180,197,227,253$, $270,273,292,322,355,45^{8}$

Charles II, i, 343 ; ii, $53,56,57$, 6I, III, I47, 239, 34I; iii, I4, $197,206,227,270,272,317,322$, $379,404,417,434,44^{\circ}$

Charles, -, iii, 125

Charlet, Rob., i, 370

Charleton, Eliz., Lady, ii, 23I ; Rich., ii, 23I ; Sir Thos., ii, 231 ;

Thos., ii, 231 : see also Carlton

Charlewood (Studham), ii, I 45 iii, 429

Charlewood (Woburn), iii, 46I

Charnock, see Chernock

Charterhouse, London, ii, 159

Chase, C. E., ii, 200; Steph., iii, I33: Thos., i, 402

Chastellon, John, iii, 422 ; Marg., iii, 422

Chaucer, Alice, iii, 403, 429 ; Mand, iii, 429 ; Thos., iii, 403, 429

Chaucombe, prior, iii, 184

Chaugrave, see Chalgrave

Chaul End (Caddington), ii, 3I 4, 374

Chaumbre, see Chamber

Chauncey, Eliz., iii, 387

Chaurugge, Will. de, iii, 408

Chaworth, bar., iii, 443

Chaworth, hon., iii, I62

Chaworth, Matilda de, iii, 439 ; Pat. de, iii, 162, 439; Payn de, iii, I62, 439, 446

Chawston, i, 384 ; iii, 180, 201, 218 , 219 ; beagles, ii, 190; man., iii, 219, 220 ; man.-house, i, 305

Chawston, Walt. of, i, 381

Cheddington, John of, $i, 373,377$ Cheeke (Cheke), Frances, iii, 28r Hen., iii, 8, 28I ; Thos., iii, 28I Cheetwood, see Chetwood

Cheiroptera, i, 138

Cheisley, - , ii, 53

Cheke, see Cheeke

Chelbert, iii, 52

Cheletone, Rob. de, iii, 427

Chellington, iii, $34,54^{-6}, 59,63$, 85 , I75; adv., iii, $5 \mathrm{I}, 55,56$; char., iii, 56 ; ch., i, $324 n$; iii, $53,54,55,56$; man., iii, 5 r. 54 , 55,56 ; pop., ii, II 5 ; rectory, iii, 54, 56 ; sch., ii, 181

Chellington Hill, iii, 54

Chellington-with-Hinwick, iii, 175

Chelmarsh (Salop), ii, 35I

Chelvinton, see Chellington

Chelvynton, see Chellington

Chelyngton, see Chellington

Chenemondewiche (Biggleswade) ii, 201 


\section{INDEX}

Cheney, Lady Jane, iii, 382, 440, 447 ; Ld., iii, 439 ; Ld. Hen., iii, $44^{\circ} \mathrm{n}$

Cheney, Alex. de, iii, I33: Anne, Lady, iii, 346, 436; Anne, iii, 445: Barth, de, iii, 133; Eliz., ii, $243,345,363$; iii, 5 I, 77 ; Geo., ii, 359: Sir Hen., iii, 440, 445; Hen., iii, 311 , 346; Jane, Lady, ii, 377 ; iii, 445 ; Jane, iii, 346 : Sir John, ii, 32I ; iii, I 9 , 272 : John (de), iii, 66, 133, 458 : Kath., see Pabenham; Lawr., ii, $243,345,382$; iii, 77 , I9I ; Margery, ii, $385 n$; Mary, ii, 363 ; Rob. (de), ii, $359,363,366$; Sir Thos., ii, 377,385 ; iii, 5 r, 77,3 I I, $346,380,381,436,440$; Thos., ii, $40,359,363,385,387$; iii, 191, 231, 286; Walt. ii, I44; Sir Will., iii, I9I ; Will., iii, 77

Cheneys, man., iii, 77,78

Cherleton, see Charleton

Cherlewude, West, see Charlewood (Studbam)

Cherneton, see Cardington

Chernock (Charnock(e), Charnok), Sir Boteler, ii, 6o $n, 62,323$; iii, 387 ; Eliz., iii, 387 ; Helen, iii, 387 ; Sir John, ii, 325 ; John, i, $335 n$; ii, 323 ; Sir Pynsent, ii, $58 n, 323$; iii, $37,3^{8} 7,436$; Rich., i, $335 n$, 377; ii, 4o, 323 ; iii, $377,386,387,388$; Sir Rob., iii, $3^{8} 7$; Rob., ii, 323 ; iii, $3^{87}$, 388 ; Sir St. John, ii, 323 ; iii, 387 ; Sir Villiers, ii, $58,59,323$; iii, $387,388,425$; Mrs., iii, 388 ; fam., ii, 60 ; iii, 338

Chesham (Bucks), adv., i, 366 ; ch., i, 319, 366, 370, 37I $n$

Chesney, Robt., see Lincoln, bps. of ; see also Cheney

Chester, earls of, ii, 28, 29; iii, 9, ro

Chester, Sir Ant., ii, 58, 59; iii, 216, 306, 434; Ant., ii, 295; Sir Chas., iii, 434 ; Chas., iii, 58 ; Sir Cbas. B., iii, 434 ; Dorothea, iii, 434; Frances, Lady, iii, 434 ; Sir Hen., iii, 434; Hen., ii, 4I, $54 n, 295$; H. M., iii, 58 ; Sir John, iii, 307,434 ; Judith, Lady, iii, 434 ; Rob., iii, 434 ; Sir Will., iii, 434; 一, iii, 433; fam., ii, 58 ; iii, 435

Chesterfield (Sandy), Rom. rem., i, 271, 273, 274; ii, 9, 10

Chesterfield, Eliz., ctss. of, iii, 427 ;

Phil., earl of, iii, 427

Chetel, iii, 5 I

Chetewood, see Chetwood

Chetham, Sons \& Biffen, ii, 200

Chetwode, see Chetwood

Chetwood, Agnes, ii, 234; iii, 384

Anne, ii, 234; Crewe, iii, $72 n$;

Dorothy, iii, 73 ; Eliz., iii, 7r, 384 ;

Guy, iii, 384 ; John, iii, $72 n$,

384 : Jonathan, iij, 72 ; Knightley, iii, 72 ; Lucy, Lady, iii, 384 Nich., iii, 384 ; Sir Rich., iii, 70 72, 73; Rich., ii, 234, 328; iii, 72 : Sir Rob., iii, 384 ; Sir Thos., iii, 71 ; Thos., iii, 384 ; Valentine, iii, 72

Chetwood-Aiken, Constantia E., iii, 72 ; John C., iii, 72, 73

Chew, Thos., iii, 35I ; Will., ii, 179 ; iii, 351 ; fam., iii, 355

\section{Cheyne, see Cheney}

Cheyney, see Cheney

Chibnall, Thos., iii, 246; see also Chubnoll

Chicheley, Hen., ii, 157; iii, 92,

I $82,3 \pm 7,424^{\circ}$ Thos,

Chichesane, see Chicksands
Chichester, Audrey, ctss. of, ii, 347, 382 ; Fran., earl of, ii, $347,3^{82}$

Chichester, Ralph de, iii, $45^{8}$

Chickessaund, see Chicksands

Chicksands (Chichesane, Cudessane, Chikesond, Chickessaund), ii, 43, 260, 266, 271 ; iii, $227 n$; char., ii, 276 ; ch., i, $315 n, 324,392$, $393 n$; ii, 273-4, 276; mans., i, 393 ; ii, 271, 273, 275, 342, 344 ; mills, ii, 27 I ; pop., ii, II 3 ; vicarage, i, $316 n$

Chicksands Priory, i, 31 3, $318 n, 349$, $356,365 n, 378 n, 389,390-93$, $395 n$; ii, $83,152,197,203,206$, $244,266,267-9,27 \mathrm{I}-8,288,289$ $293,302,303,305,336,341,343$ iii, $24,137,139,166,168,169$, 240, 242, 260, 293 ; chap, ii, 273 274, 275: Dayre House farm, ii, 275 ; mills, ii, 275 ; ii, 274 ; priors of, $i, 314,393$; ii, 203, 244, 343 ; iii, 258 ; seal of $i, 393$; 'Warming Pan Bed ' in, ii, 275

Chigwell Hall (Essex), iii, I $46 n$

Chikesond, see Chicksands

Child, Hen., i, $319 n$; John, iii, 9 90, 91, 258; Marg., iii, 82, 86 Rich., iii, 82, 83, 86, I19; Rob. le, iii, 91, 221 ; Thos., iii, 184 , 221 : Will. le, iii, 91

Chillington, see Chellington

Chiltern, Eliz., i, 390

Chiltern Green, ij, 348, 374

Chiltern Hills, i, I75, I 88 , I 89 : iii, $3^{8} 3$; earthworks, i, 269

Chinale, Will., iii, 1 I 8

Chinnor (Oxon.), ii, 45

Chippenham, man. (Suff.), i, 393

Chishull, Barth., ii, 220; John, ,ii, 220

Cholmeley, Christiana, Lady, iii, 430 ; Sir Rog., lii, $43^{\circ}$

Christadelphians, iii, $3 \mathrm{I}$

Christ Church Coll. (Oxf.), ii, 332 ; iii, 242

Christendome, Andrew, ii, 323

Christie (Christy, Chrysty), Alice iii, 25; Anne, iii, 25; R. N. ii, 198; Thos., ii, 58 , 59 ; iii, 25 , 29, 32, 33; 一, ii, 10 ; iii, 20

Christine, St., i, 358

Christmas, Rob., iii, 403, 429

Christ's Hosp. (London), ii, 290 ; iii, 301

Christy, see Christie

Chrysty, see Christie

Chubnoll, Alice, iii, II6; John. iii, I 6 ; see also Chibnall

Church, John, iii, I22; Kath., iii, 332 : Will., iii, 332

Church Close (Podington), iii, 82

Cburch End (Barton-in-the-Clay), ii, 308

Church End (Biddenham), iii, 36

Church End (Colmworth), iii, 186

Church End (Eversholt), iii, 375

Church End (Flitwick), iii, 284

Church End (Haynes), ii, 338

Church End (Knotting), iii, 139

Church End (Milton Bryant), 418

Church End (Old Warden), iil, 25 I

Church End (Ravensden), iij, 209

Churcb End (Renhold), iii, 214

Church End (Studham), iii, 426

Church End (Wilden), iii, 226

Church End (Wootton), iii, 33 I

Church End Farm (Eversholt), iii, 375

Church End Farm (Wilden), iii. 223

Church endowments, i, 324, 332

Church Farm (Podington), iii, 80

Churchill, Eleanor, iii, I28; Flectwood, iii, 125, 127 ; Jos., iii, 125 : Mrs., iii, 125
Church Panel (Shillington), earthworks, i, 276 ; ii, 294 n

Cimmay, Gilb. de, i, $315 n$; ii, 356

Cioches, Gunfrei, iii, 85

Cirencester, Bert. of, ii, 6; Rich. of, ii, 6

Cistercians, $i, 322,349,351,361-72$, $382,383,392,395 n, 399 n$; iii, 318,459

Civil War, i, 314, 342; ii, 4I-54, 340 ; iii, 4

Claidich, John de, ii, 263; Warin de, ii, 263

Clanfield (Oxon), i, 357

Clanricarde, Ld., ii, I94

Clapham, ii, $21,43,52$; iii, 123 I 26, I $47, I_{50}$, I51, 183, 279, 297 adv., i, $3^{84}$; iii, 131,132 ; chap., i, $315 n, 318,347 n, 382,383$. char., ii, $182 n$; iii, 132 ; ch., iii, 131, 132, 152, 153; deanery, $i$, 347; Fox and Hounds Inn, iii, 128 ; ind. iii, 128 ; man , iii, 128 129 , 183; man.-house, iii, 128 ; Nonconf., iii, 128 ; place-names, iii, 128 ; pop., ii, II 5 ; iii, 132 sch., ii, I82; sundial, iii, 128 ; vicarage, iii, 132

Clapham, Mabel de, iii, 302

Clapham Greenacres (Fitzjeffry's), man., iii, I29, I30, I31, I52 ; mills, iii, 130

Clapham Pk. (Bedford), ii, $66 n$, 194, 195; iii, 128

Clare, Gilb. de, ii, 384; iii, 36 ; see also Glouc., earl of; Isabel de, ii, 306, 351, 352, 353, 384; John de, iii, 173; Marg. de, ii, 284, 384 ; iii, 36 ; Rich. de, i, 394 ii, 384 ; iii, 36,135 , 198; Rog. de, see Hertford, earl of; Thos. de, ii, 384 ; Will. de, i, 315n de, fam., i, $315 ;$ iii, 78

Clare Coll. or Hall (Camb.), i, 184 ; ii, 228,229

Claremont, Alice de, i, 394

Clarence, Geo., duke of, iii, I6

Clarke (Clark), Anne, ii, 28I, 286 ; iii, I 59 ; Chris., iii, 293 ; Dorothy, iii, 293, 376; Edm., iii, 126 . Edw., iii, 293 ; Ernest, ii, 140 ; Sir Fran., iii, 293 ; F. A., iii, 29 ; Rev. Hamlet, i, 71 ; Jane, iii, 215; John, ii, 281, 285; iii, 57 , 288,318 ; Judith, ii, 281 ; Nich. iii, 376 ; Paine, ii, 55 : Sibella, ii, 281 ; Thos., ii, 281; iii, 250, 293. 456 ; Will., iii, $217,311,331 n$, 335 ; - Miss, iii, 57; see also Clerke

Clavel, Walt., i, $390 n$

Claver, Thos., i, 338 n

Clavering, Ada, ii, 238 ; Rob., see Peterborough, bp. of

Claybrookes, man. (Harrold), iii, 67

Clayton, Anne, ii, 385 ; Eliz., ii, 385 ; Frances, ii, 385 ; John., ii, 385; Marg., ii, 385; Will., see Sundon Ld. : - Miss, iii, $255 n$

Cleat Hili, ii, 194 ; iii, 209, 210

Cleat $r$ (Cleyture) (Cumb.), ii, 160

Clech, Rob. le, iii, 76

Clement, St., ii, 38 n

Clements, - , iii, 18

Clerke (Clerk), Anne, iii, 295 : Columba, iii, 29 ; Eliz., iii, 58 ; Sir Fran., ii, 4I, I80, I82 $n$; iii, 292, 295-6, 332 ; Fran., iii, 326, 332 ; Gilb., iii, 43 ; John., ii, 40, 316; John D., iii, 58 ; Julia, iii, 29 ; Ralph, iii, 29 ; Thos., iii, I28; Will., ii, 20 ; see also Clarke

Clermont, earl of, ii, I92, 193

Clermont, Alice (Alicia) de, iii, 9o, $95,135,142,1_{43}, 1_{57}, 1_{60}$ 


\section{A HISTORY OF BEDFORDSHIRE}

Cleveland, earls of, iii, $38 \mathrm{r}, 43^{8}$; Thos., ii, 42, 43, 56, 261 ; iii, $337,346,44^{\circ}, 44^{\mathrm{r}}, 445$; Thos., Ld., iii, $380 n$

Cley, Eliz., iii, 443

Cleyture (Cumb.), see Cleator

Clifford, Isabel de, ii, 23I, 232 ;

Rob. de, ii, $23 I$; Rog. de, ii, $23 I$, 232

Clifton (Clistone), ii, 260, 266, 270, 276 ; adv., ii, 279, 280 ; almshouses, ii, 280 ; assessment, ii, 26r ; char., ii, 280 ; Chicksands Priory lands, ii, 266; ch., ii, 278 , 279; coins, i, I73; mans., ii, $276,277,280,305,323,362$; iii, 260, 318; mills, ii, 276,278 , 280 ; iii, 258 ; Nonconf., ii, 280 pop., ii, I13 ; Rom. rem., ii, 6 : schs., ii, 182, 280; ship-money, ii, 261

Clifton, hund., ii, 18, 72, 113, 260305,308

Clipsham, Will., iii, 427

Clipstone (Clipston), iii, 400 ; chap., iii, 4I 4 ; man., iii, 403

Clipstone Brook, iii, 383, 399, 421

Clistone, see Clifton

Clopeham, see Clapham

Clopelle, see Clophill

Clopham, see Clapham

Clophill (Clopelle), i, 352, 397 ; ii, $66,76,306,320-5,329,342$; iii, 50, $113,313,318,351,352$; adv., ii, 324, 325; Beadlow Farm, ii, $32 \mathrm{I}$; Brickwall Farm, ii, 320 chant., ii, 325 ; char., ii, 325 : chs., i, 3I5 $n, 318,334,351 n$, 352 ; ii, 320, 324, 325; earthworks, i, 291-2, 297; ii, 321 fishery, ii, 323; Flying Horse Inn, ii, 320 ; man., ii, $32 \mathrm{I}-4$; iii, 273 ; mills, ii, 320 ; Nonconf., ii, 325 ; pop., ii, II $_{4}$; rectory, $i$, 352 ; sch., ii, I82; vicarage, $i$ $316 n, 352 ; \mathrm{ii}, 325$

Clophill Agricultural Societies, ii, 142

Clopton, Avice de, iii, $440 n$ Steph., ii, $335 n$; Will. de, iii, 440 n

Clothworkers Co. (Lond.), ii, 299

Clutterbuck, Rev. Hen., iii, 304 一, ii, 4

Clynton, Ld., iii, 422

Clynton, Joan de, ii, 360 ; Sir John de, iii, 285 ; John de, ii, 360

Clyve, Steph. atte, iii, 458

Clyve, forest, iii, $63 n$

Cnut, king, ii, 20, 2 I

Coal, i, 2, 27; iii, 5

Cobbe (Cobb), Agnes, iii, Irg; Anne, iii, 91 ; Eliz., iii, 91 ; Geo., iii, 9I, 94 ; Rev. H., if, I $8 n$; John., iii, 91 ; Paul, ii, 58, 59; iii, 91, 94 ; Rebecca, iii, 91; Thos., iii, 9I, 94-5, Irg ; Will., iii, 9r, 94 ; Dr. ii, I69, I70 ; - ii, 356, 382

Cobbitt, Nich., iii, 316

Cobden, Rich., ii, 65

Cobham, Joan, baroness, ii, 227 Lds., ii, $224 n$; iii, 188,363 ; Geo., iii, $140,187,208,230,236$ Hen., iii, 208; John Brook, iii, $I_{40}, 187,230 n$; Thos., iii, $1_{40}$ : Will., iii, 187,208

Cobham, Joan de, ii, 227 ; Reg. de, iii, $22 \mathrm{I}$; fam., ii, 262

Cochepol, see Cople

Cockayne (Cokayne, Cokkyne), Anne iii, 452; Beatrice, ii, 354 ; Chad, ii, 216 ; Edm., ii, 216,354 ; Edw. ii, 218; Eliz., ii, 206, 216, 218 . iii, I29; Frances, ii, 355,356 ; Hen., iii, 129 ; Humph., ii, 216 .
Cockayne-( cont.)

Joan, ii, 354; Sir John, ii, 218 ; John, ii, 2I5, 216, 218, 354; iii, I37; Judith, ii, 2I6; Kath., iii, 247 ; Lewis, ii, 216; Marg., ii, $216 n$; Reg., ii, 216, 354 ; Rich., ii, 216 ; Sam., ii, 216 ; iii, 247 ; Susan, ii, 216: Thos., ii, 206 ; Sir Will., iii, 452 ; Will., tii, 216 , $218,355,356$; fam., ii, 44,218

Cockayne-Cust, Fran., ii, 218 ; Hen. F., ii, $66 n$

Cockayne Hatley (Bury Hatley, Hatley Port), ii, 96, I97, 201 , 215-18, 257; adv., ii, 216n, 2I8; char., ii, 2 I8; ch., i, 3 I $5 n$. ii, 216; Hill House, ii, $216 n$; man., ii, 2I5, 216, 256; mills, ii, 2I5, 216 ; pop., ii, II3

Cocke (Koc), Eliz., iii, IOo; John, iii, roo; Rich. rii, 98

Cockeswall, man., iii, 370

Cockett, see Cokett

Cocks, Jas., iii, 368 ; Thos. S. V., iii, 368

Coddenham, Eliz., iii, I77; Hen., iii, I77

Codicote (Herts), ii, 232

Codington, John de, ii, $224 x$

Codon, Eliz., iii, 248; Rob., iii, 248

Coembestunae, see Kempston

Coffins, iii, II6, 266, 327, 334, 4II, $420,425,434,446$

Cogan, Ann, iii, 198 ; Ant., iii, 198 . Kath., iii, 198; Sara, iii, 198 Coggepole, see Cople

Coins, i, 190, 270 ; ii, 73, 26r ; iii, 2, I32, 275 ; A.S., i, 190 ; Brit., i, 170, 173, 174; ii, 210, 234, 237, 242, 26r, 304, 326, 348; Rom., i, I $73,269,273,274 ;$ ii, 2, 5-11, I3-15; iii, $175,238,390,44^{8}$

Cokerel (Cokrel), Pet., ii, 278 ; Rob., iii, 196

Coker Way (Leighton Buzzard), iii, 405

Cokett (Cockett, Coket), Ant., iii, 165, I84, 197; Hen., iii, 201

Cokkyne, see Cockayne

Cokrel, see Cokerel

Colbeck, Bridg., ii, 253; John, ii, 253

Colburn, Hen., ii, 86

Colby, Dorothy, iii, 236; Thos., iii, 236

Colchester, Brit. coins, i, I73

Coldbatch, Eliz., Lady, iii, I32 ;

Sir John, iii, 132

Cold Brayfield (Bucks), iii, rog, 28I, 304

Harbour (Dagnall), Rom. rem., ii, 6

Coldnorton, man., i, 374

Cole, Eliz., ii, 385 ; Isabella, iii, $46 \mathrm{I}$; Mich., iii, $335 n$; Rob., iii, $46 \mathrm{I}$; Sim., iii, $233,46 \mathrm{r}$; 一, iii, 375

Coleman, fam., iii, 277

Coleoptera, i, 7 r-78

Coles (Cranfield), iii, 278

Colesden, iii, 2 I9; man., iii, 220

Colesden, Jordan de, iii, 221 ; Sim. de, i, 386 ; Will. de, iii, 22 I

Coleshill (Warws.), i, 360

Colestone, Will., i, 402

Colfox, Rich., iii, 59

Colhoun, see Calhoun

College manor (Northill), iii, 250

Colleton, Chas. G., iii, 66 ; Mrs., iii, 66

Collie, Miss, ii, $x_{7}$ ?

Colling, see Collins

Colling End Green (Harlington), iii, $38 \mathbf{1} n$
Collins (Colling), Ann, iii, 31, 209 ; Barwell, ii, 252; iii, 209; Eliz., iii, 209: Sir John, ii, 59; Rob. iii, 209 ; Sam., iii, 57 ; Thos., iii, II 3

Collison, John, ii, 28ז $n$

Collop, Ann, iii, 287 ; Reg., iii, 287

Colmeworde, see Colmworth

Colmorde, see Colmworth

Colmorthe, see Colmworth

Colmworth, ii, 44, 133; iii, 31, 34, I65, I80, I86-9, 205, 271 ; adv., iii, I87, I89: char., iii, 189 ; ch., i, 385 ; iii, I86, I 88 , I 89 ; earthworks, i, 305 ; man., iii, I8I, I86-7, I89, 199; man. farm, iii, 186 ; Nonconf., iii, 186 ; placenames, iii, I86; pop., ii, II3 ; sch., ii, 182

Colmworth, Will. of, i, 385 ; see also Compworth

Colne, riv., i, 15 I

Colne, Will., ii, 224

Colnworth, see Colmworth

Colt, fam., iii, 247

Colville, Sir Chas., ii, 68 ; Joan, iii, r9r, 194; John, iii, 247 ; Maud, iii, 247 ; Sir Will., iii, I9I ; Will., iii, $\mathrm{r} 94$

Colworth (Sharnbrook), iii, 88, 92, 94 ; man., iii, 92 , 95 ; pk., iii, 89

Colworth House, iii, 88, 92, 94

Colworth Thicket, iii, 94

Coly, Eleanor, iii, $97-8$

Colyn, John, ii, 227

Combe Park (Lidlington), iii, 305

Combes Park (Ampthill), ii, 145

Common Farm (Lidlington), iii, 305

Comond, see Colmworth

Compass Inn (Ampthill), iii, 268

Compton, Eliz., Lady, iii, 208; Ld. Alwyne F., ii, 70 ; Will., Ld., see Northampton, earl of

Compton, Sir Hen., ii, 336 ; Jas., iii, 208; John, ii, 29r ; Spencer, iii, 208; Sir Will,, ii, 267, 322 ; fam., iii, 205

Compton Grove (Willington), iii, 264

Compworth, Thos., iii, I33; see also Colmworth

Comyn, Eliz., ii, 223 ; Joan, iii, r93 Conger Hill (Toddington), earthwork, i, 286, 287

Congregationalists, ii, $24 \mathrm{I}, 258,27 \mathrm{O}$ $27 \mathrm{I}, 299,374,375$; iii, 30,64 $68,96,132,202,218,279,367$ $383,415,45^{8}$

Coningsby, Eliz., ii, 360 ; Hen., ii, $347 n$; John, ii, 360

Conington (Hunts), ii, 2 I $_{4}$

Connolly, Lady Anne, ii, 277, 278 ; iii, 44I; Lady Louisa, iii, $44^{6}$; Thos., iii, 346, 44I, 446

Conquest, Alice, iii, 291 ; Benedict, iii, 29I ; Benj., ii, 59; Sir Edm., ii, 339 ; iii, 291 ; Edm., ii, 329 , 332 ; iii, 29I ; Eleanor, ii, 33I ; Eliz., iii, 29I, 295; Geoff., iii, 290 ; Hen., iii, 291; Isabel, iii, 295: Joan, ii, 329: John, iii, I29 $n, 290,29 I, 294 n, 295$; John T., iii, 291 ; Lewis, iii, 292 . Mary, iii, $292 n$; Miles, iii, 29I $n$ Sir Rich., ii, I8o ; iii, 291 ; Rich. ii, 43,54 ; iii, $270,291,295,296$; Rog., iii, 291 ; fam., iii, 289

Conquest Bury, man., see Houghton Conquest

Constable, Chas., iii, 255 ; Dorothy, ii, 233

Constable Lands (Gilden), iii, I 79

Conygrave, Joan, ii, 282 ; Reg., ii 282

Conyngestone, Nich., ii, $296 x$ 


\section{INDEX}

Cooke (Cook), Alice, iii, 375; Ant., iii, 168; Hugh, ii, 385 ; iii, 375 Jas., iii, 3II; John, iii, 248 ; Jos., i, I69; Nich., iii, 246 ; Rich., ii, 27 ; Thos. J., iii, 394 ; Rev. -, ii, I33; fam., iii, 361

Cook's Farm (Northill), iii, 246

Cook's Lane (Southill), iii, $26 \mathbf{I}$

Cookson, Thos., iii, 26I ; Dr., i, $342 n$

Cooper, Anne, ii, 385; Maj. Cooper, i, 185; ii, 8; Eliz., iii, $44 \mathrm{I}$; Eliz. C., iii, 446 ; Eliz. G. iii, 44I ; John, iii, $60,44 \mathrm{I}$ Judith, iii, 60 ; Rev. Oliver St. J. ii, 97, 98; iii, 60, 69; Sam., iii, 33, 109; Tomkinson, ii, 385 Will., iii, 44I, 446; Will D., iii, 444

Cooper's Hill (Ampthill), iii, 269

Coops (Battlesden), iii, 343

Cope, Sir WaIt., iii, $72 n$

Cople, ii, 44, 66, 96, 196, 197, 340 : iii, $32,182,193,227,23^{8}$; adv., i, 393; iii, 242 ; char., iii, 242 ch., i, $315 n, 392$; ii, 92 ; iii, 238 239, $240 n, 24 I, 242$; man., iii, 240 ; pop., ii, II6; Rom. rem. ii, 5,6 ; iii, 238 ; sch., ii, 182 . vicarage, i, $316 n, 341$; iii, 238

Cople (Copley), Anne, ii, 355 ; Edw. ii, 267 ; Humph., ii, I79; Rog. ii, 355 ; Thos., i, 384 ; fam., iii, 197

Cople House, ii, $66 n$; iii, 238

Cople Woodend, ii, 42

Copley, see Cople

Copmanford, Jos. of, i, 386

Coppin, Anne, ii, 317; Sir Geo. ii, 317 ; Jobn, ii, $317,319,320$ 377-80; John R., ii, 3 I 7 ; Martha, ii, 320 ; Sam., ii, 317 ; Thos., ii, 31 7, 320; fam., ii, 377

Coppinger, Walt., iii, 244

Copple-cum-Keone, man., ii, 145

Coprolites, i, 39; ii, II

Copt Hill land (Heath and Reach), iii, 4I 7

Corbet (Corbett), Sir Andr., iii, 4I6, $422 n$; Andr., iii, 405; Anne, iii, 405; Eliz., iii, 405, 422; Sir Rich., iii, 405; Rich., iii, 405; Rog., iii, 405; Sir Vincent, iii, $405 n$

Corker, John, iii, 245

Corn, price of, iii, 3

Corner, Anora, iii, 22o ; John, iii, 220

Cornewayle, see Cornwall

Cornish, Sir Sam., iii, 90, 95, 33

Cornwall, earls of, iii, 362 ; Edm., iii, I54, 362 ; Rich., i, 362 ; iii, 291, 362,383

Cornwall, Constance de, i, $388 n$; Sir Geoff. de, iii, 3I 7 ; Sir John (de), ii, $35,36,324$; iii, 270,272 , $273,292,296,310,317,318,319$, 326, 437 ; Phil. de, i, $388 n$

Cornwallis, Eliz., ii, 289; iii, 97 ; John, iii, 22, 348; Lucy, iii, 46, 96: Sir Will., iii, I4, 97 ; Will., ii, 289 ; iii, 46,96

Coronation almoner, iii, I2, I3, $I_{4}, I_{5}$

Corpus Christi Coll. (Oxf.), iii, 154,167

Corpus Christi gild (Eaton Socon), iii, 201

Corpus Christi gild (Leighton Buzzard), iii, 4I5

Corston, - ii, I2I

Cosen (Cosyn), Edm., iii, 211 ; Edw., ii, 244 ; Rich., ii, 2 I I

Costentin, Geoff. de, ii, 248 ; Hen. de, ii, 248
Costin, John, iii, 32

Coston, - ii, I 70

Cosyn, see Cosen

Cotes, man., see Cardington Cotton Find

Cotes, Gilb. de, iii, 3 I

Cotgrave, Thos. de, ii, 275

Cothemannesholme (Oakley), iii, I 50

Cotherstoke, Will. de, iii, 30

Cotton, man., see Cardington Cotton End

Cotton (Coton), Eliz., Lady, iii, 286 ; Eliz., iii, 286 ; Frances, iii, 286 Hen., iii, IOI ; Jane, iii, 286 ; Sir John, ii, I80, 2II, 2I 4, 2I 5 iii, 286; John, i, 396; ii, $45 n$; iii, I49: Mary, ii, 332; ini, 286 ; Philadelphia, Lady, ii, 357; Sir Rob., ii, $45 n$, 2 I I $n$; Sam., iii, 83 ; Sir Thos., ii, $45 n, 357$

Cotton End, iii, 32, 227, 234, 238 ; man. house, i, 306; iii, 234 ; Nonconf., iii, 234 ; sch., iii, 234

Conci, Ingelram de, see Bedford, earl of ; Isabella de, ii, 39

Coulthirst, Nich., ii, 316

Councell, Hugh, ii, 270

County Council, ii, I 41 I, I 42 ; iii, 320 Coupol, see Cople

Courtney, Alice, iii, 436, 437 ; Edw., iii, 436

Court Place (Wymington), iii, II 9

Coutances, bps. of, i, 313 ; iii, 54, $85,90,95$, IIO, II 4 , II 9 , I 25 . I $33, I 39, I_{42}, I_{57}, 162,175$

Couture, La, abbey of (Le Mans), i, $315 n$; iii, 439, 444, 446

Coventry, Hen., ii, I95, 254

Coventry and Lichfield, bp. of, Walt. de Langton, ii, 224, 225, 227, 228, $23^{8}, 262,263$

Covington, 11,72 ; iii, 31

Covington (Covyngton), A., i, I07I43; Hugh, iii, 64; John, iii, $2{ }_{3}$; Nich. de, iii, I54 $n$

Covington Fee, man., see Howe and Pertenhall

Cowe, John de, iii, 55

Cowepyll, see Cople

Cowper, ctss, ii, I $82 n$; Anne, ii, 327 ; Katrina C., iii, 233 ; earls, ii, I 42, I 46, I 76, 327 ; iii, 229, 233

Cowper, John, ii, I 51, I 56, 263 Mich., ii, 263; Will., ii, 314; see also Cooper

Cowslade, John, iii, 407, 430 Thos., iii, $407,43^{\circ}$

Cox, Jas., ii, IOI; -, ii, 333 ; iii, 444
Coxed

College ii warde

Coynte, Alice (le), ii, 297, 334 iii, 252; Joan de, iii, 252; Margery de, iii, 252; Will. (le), ii, 297, 334 ; iii, 252 ; fam., iii, 253

Cozens, John, iii, $25^{\circ}$

Crabbe, Helen, i, 390

Cracheley, John de, i, 320, 32 I, 325

Cracroft, Rich., iii, 196

Cradock's Field (Leighton Buzzard), iii, 402

Cramavilla, Hen. de, ii, 223, 224

Cranesle, John, ii, $316 n$

Cranfield (Crangfeld, Cranfelde), ii $2 \mathrm{I}, 25,66,77,78,8 \mathrm{I}, 90,187$, 268 ; iii, 40, 267, 275-79, 297 ; adv., iii, 278 ; agric., $\mathrm{ii}, 82,84$ 99: Black Death, ii, 88 : char. iii, 278,279 ; ch., i, 31 3 ; iii, 277 ; man., i, 312, 313; ii, $79,80,87$ 9 I, 143 ; iii, 276 ; Nonconf., iii 275, 279 ; pk., iii, 276 ; pop., ii I 5 ; priests' stipend, i, 336; Rom rem., ii, 6 ; iii, 275 ; sch., ii, I82 ; iii, $27^{8}$
Cranfield (Craunfeld, Craunfield), Jas., iii, 276 ; Sir Lionel, iii, 276 ; Rich. de, iii, 246; Will. de, iii, 276

Cranfield Court, iii, 276

Cranfield Mead (Tingrith), iii, 382 $43^{8}$

Cranford Mead, ii, $376 n$

Crangfeld, see Cranfield

Cranmer, Thos., ii, 38

Cransley (Northants), $\dot{\mathrm{I}}$, I $8 \mathrm{I} n$

Cratford, Chris., iii, 2I 2

Craunfeld (Craunfield), see Cranfield

Cravenherst, see Gravenhurst

Crawelai, see Husborne Crawley

Crawley, brook, ii, 7I

Crawley, man., see Husborne Crawley

Crawley, Arth., ii, $349 n$; Dionisia de, iii, $396 n$; Edw., ii, $346 n$, 375 ; Sir Fran., ii, 43, 364, 373 ; Fran., ii, 147, 197, 346, 356-9, $361-2,373-4$; iii, 105, I09, 137 , I 77 ; Joan, ii, 364 ; John, ii, 40, $349 n, 35^{8-9}, 361-3,366$; iii, 105, I77: John S., ii, $66 n, 319$; Judith, iii, I05 ; Rich., ii, 40, 356-7, 361, 364; Sam., ii, $66 n$; Susannah, iii, 177 ; Thos., ii, 3 I2, $34^{6} n, 356, \quad 35^{8-9}, 373,375$; T. S., ii, $356,357,374 n$; Will., ii, $35^{8}, 364,375$; - , ii, $35^{8}$; iii, 21 ; fam., ii, 66 ; iii, 36 I

Crawley House (Husborne Crawley), iii, 394

Crawley Park Farm (Husbornc Crawley), iii, 394

Crayford, Sir Will., ii, 40 ; iii, 322

Creaker (Creuker), Geoff., iii, I8z ; John, iii, 257

Creakers (Crewkers, Westende), man., iii, I81, I83, 184

Creakers, The (Gt. Barford), earthwork, i, 303

Crendon (Bucks), man., ii, 253

Crespinges, Eliz. de, ii, 276 ; Will. de, ii, 276

Cressy (Cressie), Isabel de, iii, 426 ; John, ii, $307,351 n, 352,355$, 359 ; Rog. de, iii, 426 ; Thos., ii, 352 ; Val., iii, $4{ }^{I} 4$

Creuker, see Creaker

Crevecoeur (Crevequer), Agnes de, iii, 452; Anne, iii, 183 ; Eleanor de, iii, 452 ; Geoff. de, iii, 183 . Hamon de, iii, 452 ; Isolda, iii, 452 ; Jas. de. iii, 183 ; John de, iii, I83, 211 ; Mabel de, iii, 452 Matilda de, iii, 452 ; Rob. de, iii 183; Steph., iii, 183, 211, 212 ; Will. de, iii, 452

Crewe, Lds., iii, $73 n$; John, ii, 34 I

Crewe, Temperance, ii, 34 I $n$; iii, $73 n$; Sir Thos., ii, $34 \mathrm{I}$

Crewkers, man., see Creakers

Crichton, Rob., iii, 3 II

Crispe (Crisp), Anne, iii, I 45 ; John, iii, 145

Crispin, Miles, iii, 105, I29, I 47, I 50 Croft (Crofte), John, iii, I 28 ; Hugh de, iii, I73; Will., ii, I6I

Crofts, Arabella, ii, 377, 379 Briers, Lady, ii, 377, 379 ; Edw. iii, 347; Sir Hen., ri, 377 ; Sir John, ii, 377 ; iii, 346 ; Marg., Lady, iii, 346 ; Sam., iii, 346

Croiser, see Croyser

Croisores, see Croyser

Crokebarowe, Agnes, iii, 28I

Crokedayke, Alex. de, ii, 256, 257 ; Isabel de, ii, $257 n$; John de, ii, 257 ; Juliana, ii, 257

Crompton, Eliz., iii, 283 ; John, ii, 291 ; Lovetus, iii, 283 ; Rob., iii, 283 ; Thos., iii, 283

Cromwell, Ralph, Ld., iii, 272 


\section{A HISTORY OF BEDFORDSHIRE}

Cromwell, Anne, iii, I93: Ida de, ii, 23I; John de, ii, 23I ; Mary, iii, 155; Sir Oliver, iii, 155: Oliver, ii, 45-7, 49-52, 54-5, I 79, 271, 275; iii, 4, 64, 349; Rich., ii, 55,228

Crook, John, i, 341, 345

Crools Farm (Willington), iii, 262

Crophill, Ralph, iii, 285

Croson, Eliz., iii, 274

Crosse (Cross), Hammond, ii, 363 ; Hen., ii, 200 ; John, ii, 363; iii, 269, 275 ; Thos., ii, 363

Crosse Ash (Ridgmont), iii, 321

Crosseland Fosse (Kempston), iii, 297

Crosses, i, $373 n$; iii, $47,54,76$, $100,108,127,142,148,152,234$, $279,314,315,351,353,399,458$

Crosshall Farm (Eaton Socon), iii, 190

Cross Meadow (Wilshamstead), iii, 328

Croswayte, Agnes, ii, 363

Crouche (Crouch, Crowch, Crowche), Edm., ii, $37^{8}$; Mary, ii, 346 ; Nich., ii, 380 ; Rob., iii, 54, 242 ; Sam., ii, 346

Crowe, Gerard, iii, 231 ; Nich., iii, 231

Crowhill Farm (Wilden), iii, 223

Crowmarsh, ii, 25

Croxton, Dan., i, $367 n, 368 n$. John de, iii, 162, 164; Rich. de, iii, 162

Croyser (Croiser, Croisores), Anne, iii, 78 ; Eliz., iii, 78 ; Ellen, iii, 67 ; John, iii, 85 ; Nich., iii, 85 ; Sim., iii, 67,85 ; Sir Will., ii, 256 ; iii, 158 ; Will., ii, 9o, 354 ; iii, 77,78

Crustaceans, i, 91-7

Cruys, Gamaliel, iii, 456

Cryptogams, i, 55-67

Cryscyan, John, ii, 319

Cublington (Bucks), ch., i, $37 \mathrm{I} n$, 376

Cudessane, see Chicksands

Cufaude, Anne, iii, 220 ; John, iii, 220

Cukkowe, John, ii, 205

Cullebere, John, iii, 20

Culverden, Will., ii, 269

Culy, man. (Wootton), iii, 33 !

Culy Green (Wootton), iii, 329

Cumming, Will. C., ii, 252

Cunningry Close (Clapham), iii, I 32

Cuppe, Rob., i, 399

Cursson, see Curzon

Curtis (Curteys, Curtius), Adolph, iii, 206; Aubrey, iii, I18; Hugh, iii, I18, I22; Jas., ii, 265 ; John, iii, II 8 , I20; Matilda, iii, 436 ; Thos., iii, 436; Sir Will., iii, 206 Will., iii, 94

Curzon, Agnes, iii, 452 ; Greg., iii, 140; Mary, iii, 452 ; Thos., iii, 452

Cust, Eliz., ii, 216 ; Fran., ii, 216 ; Hen., ii, 216 ; Hen. F., ii, 216 , 257 ; John $\mathrm{H}$., see Alford, vsct.; Lucy, ii, 216; Sir Rich., ii, 216 ; Saville, ii, 216 ; fam., ii, 66

Cutha, see Cuthwulf

Cuthbert, --, ii, 385 ; fam., iii, 286

Cuthwulf (Cutha), i, $269 n$; ii, 18, $349 n$; iii, I, 4 O

Cutts, Chas., iii, 5 I

Cycle Works, ii, I27

D'Abernon, Eliz., iii, 78 ; Gilb., iii 78 ; John, iii, 78,79 : Will., iii, 78 Dacre, Thos., Ld., iii, 192
Dacre, Anne, iii, I92; Eliz., iii, 192; Geo., iii, I92; Mary, iii, 192

Dacre and Greystock, Thos., Ld., iii, I92 ; Will., Ld., iii, 192

Dacres, Annabella, iii, I33: Edw. iii, 133; Geo., iii, 182 ; Rob., iii, 92, 133, 182, 302 ; Sir Thos., iii, 133

Dagnel (Dagnoll), Margery, ii, 275 ; Pet, ii, 275 ; Sam., iii, 449

\section{Daie, see Day}

Daintone, see Dunton

Daintry Wood (Tingrith), iii, 435

Dairy farming, ii, 136

Dakeney, Alice, ii, 322 ; Joan, ii 321, 322, 323; Sir John, ii, 322; John, ii, 321, 322 ; Mary, ii, 322 ; Rob., ii, 321 ; Sir Rog., ii, 321 , 322 ; Rog., ii, 32I-3; Walt., ii, 322

Dalbier, Col., ii, 53

Dalderby, John, bp., see Lincoln, bps. of

Dale, Anne, ii, 207, 253; Isabel de la, ii, 207; Joan, ii, 227 ; John, ii, 207, 228, 252 ; Maud, iii, 329 Thos, le, ii, 207, 208, 227, 252 ; Will., ii, $207,227,252,253$ : - iii, 329

Dallow (Dollow), man., ii, 350, 356 $357,358,362,366$; man.-house, ii, 356 ; mill, ii, 366 ; prehist. rem., i, 145, 174; ii, $34^{8}$

Dallow Farm (Luton), ii, 357

Dalton, Capt. Rob. F., iii, I7I - , iii, I32

Dalyell, Col. J. T., ii, 70

Dam Acre (Northill), iii, 248

Dame Ellensbury, man., iii, $270 n$, 291,292

Dame Ellensbury Park, ii, 147

Dame Margaret's Holme, see Dam Acre

Dammartin, Reg. de, ii, 255

Danes, i, 28I, 3ro; ii, I8, 19, 20 73 ; iii, I

Daneswood (Woburn Sands), iii, $33^{8}$

Daniell (Daniel, Danyell), John, iii, $56,3^{8} 5$; Edw., ii, 329, 333, 335 Eliz., ii, 329 ; John, iii, $43^{8}$ Rich., ii, 329; Stepl.., ii, 329 Thos., ii, 329 ; Will., ii, 329,333

Danish place-names, i, 297

Dansey, _-, ii, I 88

Dansie, Jas., ii, 385

Danver, Eliz., see Neville; Sir John, iii, I 4

Danvers, Burnaby, iii, 364 ; John, iii, I93 ; Sam., iii, 193

Danyell, see Daniel

Dapifer, Eudo, ii, 207, 208, 243 $244,251,277,278$; iij, I90, I 91 , $199,219,229 n, 260,386 n$

D'Arcy, Sir Conyers, ii, 295

Darell, Anastasia, iii, I 54 ; Beatrice, iii, I 54 ; Isabel, iii, I54 ; John, iii, I 54 ; Julian, iii, I 54 ; Marg., iii, I 54 ; Thos., iij, I 54 ; fam., iii, I 66

Darkland (Ridgmont), iii, 32

Darley (Derb.), abbot of, i, 372 n

Darling (Derling), Sir Rob., iii, 54 ; Thos., iii, Ior

Dartford (Kent), prioress of, i, 403

Dartiguenave, Anne, iii, 197

Darwin, Bern., ii, I98

Datchet (Bucks), man., iii, 436

Daubeney, Giles, Ld., ii, 267, 322

Daubeney (Albini, Álbingi), Alice, iii, 298; Amicia, iii, 237 ; Ascelina (Asseline, Azeline) de, iiv, 236, 271, 317: Cecilia, iii, 148 ; Cecily, i, $352,377 n$; iii, 237 ; Christina, ii,
Daubeney (cont.)

321 ; iii, 271 ; Eleanor, iii, 298 ;

Eliz, iii, 298 ; Sir Giles, iii, 298 ;

Giles, iii, 299 ; Hen. (de), i, 35 I ; ii, $263,267,281,321,322$; iii, 237, 273 ; Isabel, ii, 211,321 ; iii, $236,271,317,318$; Joan, ii, 321,322 ; iii, $236,268,271,272$; John, iii, 298; Marg., iii, 298, 299 ; Nigel (de), I 43, ii, 2 I I, 263, $277,281,283,309,321,324,329$, 376,383 ; iii, $50,51,60$, I т 3 , г 46 , $196,199,236,260,271,286,309$, $315,317,326,379,396,435$; Philippa, iii, 298 ; Ralph, iii, 298 ; Rob., i, $318 n, 350,351,352,366$, ii, $26,263,270,281,321,322$, $324,325,342,344$; iii, I 48,236 , 237, 27I, 3I7, 318 ; Sim., ii, 321 : Will., ii, $32,321,322,383$; iii, II $, 27 I, 298$; fam., i, $372 n$; ii, 30, 323,324 ; iii, 273

Daubeney, bar. of, i, 292 ; ii, $30 n$, 324

Daubeny, hon., iii, I 12

Daventry, Isabella de, iii, 436 : Joan de, iii, 436, 437; Matilda de, iii, 436; Rob. de, iii, 436 ; Thos. de, iii, 436, 437 ; Walt. de, iii, 436 ; fam., iii, 286

Davey, see Davy

David I, king of Scotland, i, 388 ; iii, 9, 65

David Ashby, man., ii, 350

Davie, see Davy

Davies, Olıver, iii, 212 ; Thos., iii, $278 n$

Davis, H. W. C., ii, 26 n ; Kendrick, iii, 385 ; Stanley P., ii, I90

Davis's Spinney ('Turvey), iii, I Io

Davy, Eliz., iii, II5; Sir Humph. ii, I 35 ; John, ii, 40

Dawe, Rob., iii, $3^{85}$

Dawkins, Rev. Edw. H., iii, 233 ; E. H. F., ii, I83n; F., iii, 231 -, Mrs., iii, 233 ; fam., iii, 231

Daws, Sir Thos., iii, $3 I_{4}$

Dawson, John, iii, I 45

Day (Daye, Dey, Deye), Bonj., ii, 360 ; Edm., iii, 108, 221 ; Frank. iii, I9I ; Gilb., iii, 96 ; John H. iii, I91; Marg. le, iii, I 59 ; Sim. le, iii, I 59 ; Thos., i, 383, 385 ; Walt. iii, I59; Will., ii, 360 ; iii, 175

Deacons (Deacon), Edw, iii, 282 Mary, iij, 282 ; Thos., iii, 282 , $288,43^{8}$

Deacon's charity, iii, 4 I6

Dead-man's Slode (Slade) (Leighton Buzzard), A.S. rem., i, I 87

Dean (Dena, Dene), ii, 96; iii, 123 , I $32-36, I_{42}, I_{65}, I_{70}, I_{74}$; adv. iii, I35; chant., iii, I36; char. iii, I 36 ; ch., i, $315 n, 325 n$ 394 ; iii, I34; mans., see Overdean and Netherdean; Man House Farm, iii, 132: mills, iii, I32; Nonconf., iii, I32, I36: place-names, iii, I 32 ; pop., ii, I 5: Rectory Farm, iii, I32 : sch., ii, 182; treasure trove at iii, 132 ; value of land, $\mathrm{iii}, \mathbf{1} 34$

Dean, Nether (Lower), iii, 132

Dean, Upper, iii, I32, 160

Dean (Deane, Dene), Anstis (Anastasia), i, 355, 357: Chris., ii, 289 ; Jas., iii, IO2; -, iii, I 55 ; see also Den

Deane Hills Park, iii, 394

Deaneries, rural, i, 31 3, 32 3, 347

Dean Hall, iii, 132

Dean House, iii, 132

Dearman, Edw., ii, 325; Thos., ii, 324 


\section{INDEX}

Debenham, Gilb., iil, 37 I

Decker, Sir Matth., iii, 255

Decons (Decon, Dekene, Dycons),

Eliz., iii, 306, 309 ; Rich., ii, 328; iii, 306, 309 ; Thos. (le), iii, 306, $364 n, 407$; fam., iii, 3 I $3_{3}$

Deep Hall (Óxf.), iii, 275

Deer Pk. (Husborne Crawley), iii,

394 Grey of Wrest, see Grey (of Wrest) de

Dekene, see Decons

Delamere, Thos., i, 363

De Lanie, see Delaune

Delap, Jas. B., iii, 2 I9 ; Rev. Rob., iii, 2 I9; fam., iii, 183

Delaune (de Lanie), Gideon, iii, I83, 219 ; Will., iii, I84, 2 I9

Delavere, Hester, iii, I34; Kinnaird, iii, I 34

De la Warr, Joan, iii, 339 ; John, iii, 339

Delawne, see Delaune

Dell, Dr. Humph., iii, 286 ; Will, i, 342 ; iii, 179 ; 一, iii, 64

Delmé, Ánne, ii, 286

Den, Gilb. de, iii, 324-5 ; Will. de, iii, 324-5; see also Dean

Dena, see Dean

Dendon, Agnes, i, 398 ; Rob., i, 398

Dene, see Dean

Denel End (Dunhill), iii, 284

Denel (Dennel) End (Flitwick), iii, 288,438

Denham, Hen., ii, 357

Denis, see Dennis

Denison, Sam., ii, 204 ; Rev. Will. H., iij, 54,56

Denman, Sir Thos., iii, 72

Dennis (Denis, Dennys) Andr., iii, 25: Eliz., iii, 298, 304 ; John, iii, 298; Mary, iii, 298, 304 ; Matth., iii, 298, 304 ; Rob., iii, 277, 298;

Will., iii, 298, 304

Denny (Cambs), iii, $4^{\circ}$; nuns of, i, 326

Denny, Ant., iii, 302 ; Edw., see Norwich, earl of; Honoria, iii, 330; John, iii, 302 ; Thos., iii, 302

Dennys, see Dennis

Denshire, Geo., iii, I $55 n$

Denston Hall (Suff.), iii, 243

Denton (Lincs.), ch., ii, 217

Denton, Nich., ii, 40; Rich., iii, 361

Derby, sch., ii, I 55

Derby, Alice, ctss. of, iii, 102 ; Isabel, ctss. of, ii, 35I ; earls of, iii, I39; Edw., iii, I r9 ; Ferd., iii, I02 ; Hen., ii, 247 ; iii, I19; Thos., iii, I02, I19, 283 ; Will., ii, 35 I ; iii, 78,230

Derby, John de, iii, 30 ; Ralph of, i, 384 ; Rich., i, 384 ; Will. de, iii, $357 n$

Dere, Godwin, iii, I34

Derling, see Darling

Dert House (Eaton Socon), iii, I90

Despenser, Anne le, ii, 23 i Edw. le, ii, 230, 231, 232; Eleanor le, ii, 300, 339, 362, 384; Hugh (le), ii, $31,231,300,339,362,384$; iii, 262; Rob. le, ii, 291 ; Will. iii, 95

Deule, Alwin, i, $297 n$; ii, 276 ; iii, 155

Devenisb, Eliz., ii, 355 ; Rich., ii, 355

Devereux, Will., iii, 18

Devilhoe, see Düloe

Devil's Jumps, see Yarlswood

Devil's Spinney, see Doctor's Corner

Devon, Christian, ctss. of, iii, 290
Devonshire, lace ind., ii, I23

Devotion, Thos., see Dyer

Dey, Deye, see Day

Deyville, Joan, ii, $23^{8}$; John, ii, 238 ; iii, $190 n$

Dickens, Eleanor, iii, 380 ; Thos., iii, 380

Dickenson, - - iii, II 7

Dickinson, Capt. John Marche, ii, 69

Dickson, John, iii, r73

Dier, see Dyer

Digby, Beatrice, iii, 45 ; Sir John, iii, 45,49 ; John, iii, 46 ; Sir Kenelm, ii, 275

Dilewick, man., see Dylywyk

Dillingham (Dyllingham), Brampton G., iii, I62 ; Fran., iii, I95, 226 : Theophilus, iii, I62, 164

Dillon, Col. Rob. V., iii, 328

Dilly, Chas., iii, 257; Edw., iii, 257 ; John, iii, 257

Dimmock, see Dymoke

Dingwall, bar., ii, 327

Diocese, i, 332, 346-47

Dionisia, iii, 419

Distaff Lane (Lond.), ii, 2 I 4

Ditchfield, Edw., ii, 3Io, 312 ; ii, $253,285,292,306,321,326,332$ Dive, Dives, see Dyve

Dix, Will., iii, 80

Dixse, Hughe, iii, 200

Dixwells, man., iii, 435,438

Dobbe, Frances, iii, 67 ; John, iii, 67

Dobbin Spinney (Turvey), iii, 1 Io

Dobbs, Phil., iii, 51 ; Will., iii, 194

Dobson, Frances, ii, 94 ; iii, 456 ;

John, iii, 456; Miles, iii, 456;

Will., iii, 200, 456 ; -, ii, I69

Dockra, see Docwra

Doctor's Corner (Clapham), i, I I 6

Docwra, Edw., ii, 359, 365; Thos., ii, 59,365 ; Capt., ii, 69

Dodd (Dod), Jas., ii, 296 ; Katt., ii, 378 ; Rev. Will., ii, I02; iii, 345 ; Will., ii, 378,383

Dodsworth, Eliz., iii, 322

Dodwell, -, ii, 296

Doe, Jolland le, ii, 255

Doffevill, Hugh, iii, 257 ; Isabcl, iii, 257

Doggett, - , iii, 4I 5

D'Oilli, see Doyley

Dokesworth, Hugh de, iii, 98 ; John de, iii, 98

Doket, see Duckett

Dolby, - , ii, I 77

Dolling, Rev. - , iii, I28

Dollow, man., see Dallow, man.

Dolt, Rich., iii, 385 ; 'Thos., iii, 385

Domesday Survey, i, rgr-266

Dominican monks, see Friars, Black

Dominican nuns, i, $396 n$

Domnic, iii, $229 n$

Doncaster, Jas., vsct., see Carlisle, earl of

Donne (Done), Dinah, iii, I $q^{6}$ Jas., iii, 293; John, i, 342; iii I46, 233 ; Rog. de, iii, I37

Donton (Dontone), see Dunton

Dorchester (Oxon), bpric., i, 3 I I, 31 3, 347 ; ii, I 8, I 53 ; bps., ii, 20, $2 \mathrm{I}, 294,309$; iii, $84,408,4 \mathrm{I} 4$; St. Peter's Ábibey, i, 388 n

Dormer, Dorothy, iii, I77 $n$; Edw., ii, 319: Eliz., iii, 427; John, ii, 360,362 ; iii, 277 ; Mary, see Sydney ; Sir Rob., ii, 40 ; iii, 407 , 427, 429; Rob., ii, 354 ; iii, I 77 , 406; Susanna, iii, 427 ; Sir Will., iii, 427 ; Will, iii, 177,427

Dorset, Cecily, mchnss. of, iii, 380 ; mqs. of. iii, $38 \mathrm{r}$; Hen., iii, 89 , 380 ; Thos., iii, 89, 230,248

Dorset, Rich. of, i, 402

Douglas, Rev. Jas., i, I82
Dounton, see Dunton

Dove House Farm (Kensworth), iii, 368

Dover (Kent), hosp., iii, 395

Dover, hon., iii, 50

Dover, Hen., earl of, iii, 314 ; John earl of, ii, $342 n$

Dover (Dovre), John de, ii, 283 ; Junian de, ii, $35 \mathrm{I} n$; Phil. de, ii, 283 ; Rich. de, ii, 283

Dovey, John, i, 398

Downe, Will., earl of, iii, I40

Downes, I.t.-Col. Chas. V., iii, 387 ; Mrs. C. V. S., iii, 338; fam., ii, 293

Downham, Hen., ii, $35^{8}$

Downing, Edw., ii, 22I ; iii, 29 392, 425 : Sir Geo., ii, 256, 257 ; Sir Jacob G., ii, 256, 257 ; Marg. ii, 256

Downing College (Camb.), ii, 257

Downshire, mchnss. of, ii, 330 ; mqs. of, iii, 376

Doyle, Col., ii, 67

Doyley, Cath., iii, I88; John, iii, I 88; Rob., iii, I07, 129, I 47 I $53 n$

Drake, Eliz., iii, I98; Helen, Lady, ii, 347,382 ; Sir John, ii, 347 382 ; Thos., iii, I98; -, Capt. iii, 276

Drakelowe, Ankareta, iii, 424 ; Emma, i, 389, 390 ; Thos., iii, 424

Draper, Gabriel, iii, 407 ; John, ii, 89,234 ; Will., iii, 407

Draycot, Ant., i, 334

Dray's Ditches (Limbury), earthworks, i, 280 ; ii, $34^{8}$

Drayton (Bucks), i, 370

Drayton, Ada, iii, 300 ; Eliz., iii, 299 ; Geoff. de, ii, 252 ; Isabel iii, 299 ; Joan, iii, 299 ; Joanna de, ii, 25I; John, iii, 299 ; Miles de, ii, 25I-3; Nich., iii, 299

Drayton Parslow, ii, 254, 332

Drayton's Manor (Kempston), sec Brucebury

Drayton's Manor (Tempsford), ii, $25 I-3$

Drew's Wood (Northill), iii, 245

Drinking Bush Hill (Stevington), iii, Io I

Drivval (Drival), Godf. de, iii, I2 ; Will, iii, $223 n$

Drover's Way Field (Northill), iii, 25I

Droxford (Hants), A.S. rem., $i$, I $83 n$

Dru, Will, iii, 247

Druce, Edric, ii, 14

Druel, Amice, iii, 92 ; Giles, iii, 92 John, iii, 92 ; Will., iii, 90 ; fam., iii, 9 I

Druet, Ralph, iii, 74 ; Sampson,

iii, 74 Dryden, Sir Hen., i, $187 n$; ii, 7

Drye, Sim., ii, 379

Duberly, Sir Jas., iii, 125

Dublin, archbp. of, iii, I 9

Duck End (Maulden), iii, 275, 314 , 316

Duck End (Wilshamstead), iii, 326

Duckett (Doket), Ann, ii, 377,378 , 379 ; Noah, ii, 377 ; Pet., ii, 377 378 ; Rob. ii, 34 I

Duckford, man., see Duxworth

Duck's Cross (Colmworth), iii, I86

Dudley, Alice, iii, II2; Anne, iii, I1 ; Aug., iii, II2; Edw., iii, II 2 ; Eliz., iii, 57, I 12 ; Fran. iii, I I2; Gamalicl, iii, I I2 ; Joan, iii, I12; Rich., iii, I12 ; Sir Rob., iii, 3Io; Rob., iii, II2; see also Leicester, earl of ; Thos., iii, 57 , I I2; - , iii, 4 I 6 


\section{A HISTORY OF BEDFORDSHIRE}

Dudley Castle, ii, 364

Dudleys, man. (Turvey), iii, I12, I I3, I I 4

Dudswell (Herts), ii, 375

Duffyn, John, i, 354 n

Dugdale, J. H., ii, 14 r ; Sir Will., iii, 355

Duke, Warin, iii, 95

Duloe (Devilhoe), i, 297; iii, I93, I 97-8, 202 ; farm-houses, iii, I90

Duloe, brook (Eaton Socon), iii, 189

Dun, see Dunn

Duncombe, Anne, iii, 407 ; Brandreth, iii, 407 ; Sir Edw., iii, 344 . 383, 422; Edw., iii, 385 ; Eliz., iii, 330, 345, 405; Ellen, iii, 344, 423; Fran., iii, 51, 330 ; Sir John, iii, 345 ; John, iii, 344 ; Thos., iii, 330, 408; Will., ii, 4o, 44 ; iii, $344,345,367,405,407,4$ I5, 422 n, 423 ; fam., iii, 415,422

Dundin, John, iii, 220

Dungewood (Dungee Wood), iii, 64,67

Dunhill, see Denel End

Dunn (Dun), Tom, ii, I98; iii, 350

Dunrigge, man. (Caddington), ii,

3I5
Dunstable (Durocobrivae, Dunstaple), i, $320,328 n, 376,394$, 400,401 ; ii, I, 3, 23, 28-3I, 34 , $37,43,45,53,105,178$; iii, II, $18,290,294 n, 336,337,349$, $355-7,360,361,389,400$; adv. i, 378 ; iii, 366 ; agric., ii, 83-5, 129, 1 32, 133, 136; almshouses, iii, $351,367-8$; A.S. rem., i I75, 184, 189; assize of bread and ale, iii, 358; Brotherhood House, iii, 367 ; chants., i, 326 ii, 179; iii, 367 ; char., ii, I 79, I82 $n$; iii, $367-8$; ch., i, 17 $315 n, 321,328,335 n, 350,373$, $378,396 n$; iii, $285,351,360$, 364-6, 368-9 ; Civil War, ii, 47 . coins, i, 173 ; ii, $2,6,7$; council at, i, 373 ; crosses, i, $373 n$; iii, 35 I 353 ; crown property, iii, $27 \mathbf{I}$. deanery, i, 347, 351, 404; disputes with prior, i, 319,320 ; iii, $358-60$, 366 ; earthwork, i, 166, 269, 270; ii, 9; Five Knolls, i, I69, 270 ; forestry, ii, I44: gaol, iii, 358 gilds, $i, 330$; iii, $360,366-7$; gipsies, iii, 352; highway robberies, ii, 99, 102 ; hosps., i, 349 ; ind., ii, II9-22, 349 ; iii, 350-I, 363 ; inns, iii, $351,352,355$ $364,368,394$; Jews, iii, 363 . Katharine of Aragon's trial, ii, 38 ; Ladies' Lodge, iii, $35 \mathrm{I}$ 368 ; lepers, i, $40 \mathrm{I}$; man., i, 376 ; iii, 361 ; mkts. and fairs, ii, $46,87,88$, I02 ; iii, $35 \mathrm{I}, 36 \mathrm{I}-3$ mayor, ii, 35,89 ; iii, 360 ; mills, i, 320 ; iii, 351 ; miracle play, iii, 350 ; Mount Pleasant, i, 160 ; Nonconf., iii, 367 ; pk., iii, 350 , $35 \mathrm{r}$; parl. repre., iii, 36r; peasants revolt, i, 373 ; place-names, iii, 350-I ; poor house, iii, 352 ; pop. ii, II4 ; prehist. rem., i, I50, I59-70, I74, 269, 270 ; $\mathrm{iii}, 350$; races, ii, I9I ; rectory, iii, 366 roads, i, I76, 269 ; ii, 4 ; iii, 354 Rom. rem., i, 293, 294; ii, 2, 3 , 6,7 ; royal visits, $i, 372$; ii, 24 27 ; iii, $352,353,355$; schs., i 351 ; ii, 149, I 78-9, I99; iii, 350 $35 \mathrm{I}, 356,367$; sheriff, iii, 361 squatters, iii, 352 ; tolls, iii, 362 tournaments, ii, 30,33 ; iii, 353 354 ; town hall, iii, 351 ; value of land, ii, 92 ; vicarage, $i, 316 n$; wells, ii, 2 ; iii, 349
Dunstable, Gilb. of Tingrith, dean of, $i, 321$

Dunstable, Matth. of, i, $325 n$; iji, 49 Dunstable Downs, ii, 7, 198, 199: iii, $349,363,369,447$

Dunstable larks, iii, 363

Dunstable Priory, $i, 313,317 n, 319$, $321-3,325,332,349,350-1,353$, $362,366,371,374-5,382,39 \mathrm{I}$, $394-6,400-1$; ii, I $7,23,31-2$, $84-5,87,9$ I, I $44-5$, I 49, I 78 , $332,353,357,360,376-7,380$, 384 ; iii, $29,107,285,286,288$, $320-3,325,346,348-52,356,359$, $361,362-4,372,377,382,384$, $390,392,395,397-8,408,428$, 430-2, 442, 448-5 I, 456 ; episcopal visits, $i, 374,375$; lay brothers, $i, 374,403 n$; priors, i, 314, 320, $328,353 n, 377,378,379 n$; ii, $33,83-6,3$ I $8,328,350$; iii, $89,34^{2}, 344,35^{8}, 390 \cdot \mathrm{I}, 395^{-6}$, 427 ; sub-priors, i, $382,384 x$

Dunstan, St., ii, I54, 2 I 7

Dunton, i, 323, $335 n, 344 n$; ii, 201, 218, 233; adv., ii, 220, 222 ; char., ii, 222 ; ch., i, 315n ; ii, 221 ; forestry, ii, 146; man., ii, 221: Nonconf., ii, 222 ; pop., ii, I1 3 ; sch., ii, I82; vicarage, i, 316

Dunton Chamberlain (Newtonbury), man., ii, 219, 220, 22I ; earthwork, i, 307

Dunton Fen, ii, 222

Dunton Goyes, man., ii, 220

Duport, -, iii, 415

Durant, see Durrant

Durham, bpric. of, i, 31 2 ; ii, 220, 262 Durham, Margery, iii, 344; Col., iii, 286

Durrant, (Durant, Duraunt), Eleanor, iii, 285; John, i, 375, 394 ; iii, $285,350,354^{n}$

Dusburgh, Matilda, iii, 437 ; Ralph, iii, 437

Duxworth, man., iii, 98

Dycons, see Decons

Dyer (Dier), Cath., iii, r 88 ; Edw., iii, 385 ; Hen., iii, 188 ; Sir Jas., iii, 187; John, ii, 229, 292 ; iii, 127, 131, 315, 393, 438; Sir Lewis, iii, 156, 189, 366 ; Sir Lodovick, ii, 44 ; iii, 187 , 188; Sir Rich., iii, 156, 187, 366; Thos., iii, 3r I ; Sir Will., iii, $156,187,188,366$; Will., iii, 189

Dyers Hall Farm (Harlington), iii, 379

Dyllingham, see Dillingham

Dylywyk (Stagsden), man., iii, 13, 96-7, 212

Dylywyk, Hen. de, iii, 98

Dymoke, Isabel, iii, I29 ; John, iii, 129

Dymond, Mrs., iii, 338

Dynevor, Ld., iii, 47, 96 ; Geo., Ld., iii, 45

Dynevor House (Ampthill), iii, 268 Dyson, Eliz., iii, 427 ; Jas., iii, 195 ; Jer., iii, 427

Dyve (Dive, Dives, Dyves), Beatrice, iii, 45 ; Edm., iii, 391 ; Sir Fran., iii, 45: Fran., iii, $\mathrm{IO}_{4}$; Hen., iii, 46 ; Isabella, iii, 48 ; Sir John, i, $395 n$; iii, 45, 48, 391 ; John, iii, $46,311,355$; Sir Lewis, ii, 43-6, 48: iii, 4, 45-9, I04, 27I, 391 ; Lewis, iii, 48, 311 , 39r: Mary, iii, 48; Theophila, iii, 104; Will., ii, 150 ; iii, 46 , 39 r, 394 ; fam., ii, 58 , 60 ; iii, 37

Eadwig, mint of, iii, 2

Eagles, Marianne, iii, 275
Eakring (Notts), ch., i, 394

Ealdred, iii, 345

Eames, Jas., ii, 344

Earl Marshal, the, ii, 223, 261, 262, $301 n$

Early Close, Gt. (Renhold), iii, 215

Early Close, Little, iii, 2 I 5

Early Man, i, 145-74

Earthworks, i, 267-308; ii, 73, 210 $242,25 \mathrm{I}, 26 \mathrm{I}, 288,294 n, 32 \mathrm{I}$ $324,344,348,365$; iii, 64,102 , $104,176,202,206,207,210,212$ $215,252,262,284,320,32 \mathrm{I}, 402$, 439, 447 ; Brit., ii, 8 ; Rom., ii, 5,9 , 10

East Anglia, Helen, ctss. of, iii, 297 Gurth, earl of, iii, 297; John le Scot, earl of, iii, 297

Eastcotts, ii, I16; iii, 31, 227, 234 279 ; man., iii, 237 ; sch., ii, 182

East End (Cranfield), iii, 275

East End (Flitwick), iii, 284

Easf End (Harlington), iii, 379

East End (Wilden), iii, 223, 226

East End Moor (Flitwick), iii, 288

Eastern Association, the, ii, 43, 44 $46,47,51$

Easton (Flitwick), see East End

Easton (Hunts), iii, 123, 136

Easton (Northants), iii, 247

Easton Maudit (Northants), iii, I IO

Easton Mill (Flitwick), iii, 287

Eaton, bar., i, 386, 403; ii, 208 $243 n, 25 \mathrm{I}, 253,277,28 \mathrm{I}$; $\mathrm{iii}_{\text {, }}$ $190-4,197,219,246,258,260$, $424 n$

Eaton, Gerard of, i, 399 ; Sim. of i, 372, 377; Ulmar of, ii, 207 208, 243, 25I; iii, 248; Wildeboef of, i, 385

Eaton Beauchamp, iii, 193

Eaton Bray (Eitona, Eytona), i $320,325 n, 373$; iii, 336,369 adv., iii, 374; Bellows Mill, iii, 370 ; cast., i, 305 ; ii, 28 : iii, 369,370 ; chants., iii, 375 char., iii, 375 ; ch., i, 3 I $5 n$; iii, 369 , 373 ; ind., iii, 369 ; man. iii, 370-2, 456; mkts. and fairs, iii, 369 ; mills, iii, $307,370,372$ pk., iii, 371 : Peverell's fee, iii, 372 ; place-names, iii, 370 ; pop., ii, II 4 ; iii, 374 ; Rye cottage, iii, 369 ; sch., ii, I 82 ; vicarage, i, $316 n, 317$; iii, 369,374

Eaton-cum-Soka, see Eaton Socon

Eaton Ford, iii, 189, 190; bridge, i1, 53

Eaton Green (Eaton Bray), iii, 369 Eaton Socon, i, $326 n$; ii, 43, 71 $76,86,277$; iii, $96 n$, 180, 189 ; adv., iii, 200 : agric., ii, 136 Begwary Farm, iii, I9o; cast. iii, 191 ; chant., i, $329 n$; char., iii, 201, 202 ; ch., i, $315 n, 325 n$ 394; ii, 208; iii, I99; deanery, i, 347 i earthworks, i, 297, 298, 299 ; gild, i, 330 ; inclosures, ii, 97 i mans., i, 394 ; iii, 190, I9 I, 195, 198, 199, 201, 258; mkt., iii, I99: mill, i, 298; iii, 189; Nonconf., iii, I9o; Oakley Club meet, ii, 189 ; pk., i, 386 ; iii, 197 ; placenames, iii, 190; pop., i, 113 prehist. rem., i, $\mathbf{I}_{45}$; priory lands, iii, 197; rectory, iii, 201; sch. ii, 182

Eaton Soken, see Eaton Socon

Eayre, J. and J., bellfounders, ii, $236,245,285,292,373$; iii, 29, $63,68,116,138,152,164,167$, $179,200,242$

Ebrington, Edw., iii, 40

Ebroinus, ii, 78

Eccles, Rich., iii, 392 
INDEX

Eccleshale, Ralph de, ii, 360

Ecclesiastical divisions, i, 346

Ecclesiastical history, i, 309-48

Eccleye, see Oakley

Echam, see Higham Gobion

Edeston, ii, 364

Edgar, king, ii, I9, 21, 286, 294 ; iii, 338

Edgcumbe, Sir Rob. P., ii, 242, 244

Edgebury (Aspley Guise), iii, $33^{8}$

Edington, Will. de, $i, 402$

Edith, queen, ii, 2 I

Edlesborough (Bucks), i, 384, 404

ii, $7 \mathrm{r}$; iii, 336,432 ; ch., iii, 372

Edlesborough Hill, iii, 369,370

Edlesborough Mill, iii, $37^{\circ}$

Edmund, king, ii, I9, 20

Edmunds, Islip, iii, I 43 ; - , iii, I4 ${ }^{8}$

Edred, king, i, 3 I I $n$

Edward (the Elder), i, 28 I ; ii, I8, I9, 2 I, $25 \mathrm{I}$; iii, I, 345,4 or

Edward I, iii, 353, 362

Edward II, iii, $281,362,428$

Edward III, iii, 353, 354

Edward VI, ii, 2 IO, 275

Edward (the Black Prince), iii, ror

Edward, Hen., iii, 3 I I

Edwards, Anne, ii, 28r $n$; Eleanor, ii, $244 n$; Eliz, ii, 282 ; Frances, ii, 282 ; Geo., ii, 69, 207, 262 $280,28 \mathrm{r}, 282,285$; Geo. N. ii, 285 ; Hen., ii, 4o; Jasper ii, 207, 244, 247 ; Rich., ii, $55 n$, $244,262,265,282$; Sam. B., ii 262, 263 ; Will., i, 399; ii, $185 n$; iii, 328 ; Will. B., ii, 262 ; Will. G., iii, 122; fam., ii, 28 r, 285

Edwin, ii, 314, 36I

Edwinsmede (Turvey), iii, I 3

Edworth, ii, II3, 20I, 2 I8, 220, $223-26,248,26 \mathrm{r}, 262,357 n$. adv., ii, 226; char., ii, 226 ; ch. i, 3 I5 $n$; ii, $223,225,302$; ch farm, ii, 223 ; man., ii, 223, 224 225 ; moat, ii, 223 ; place-names, ii, 223; Rom. rem., ii, 7, 223 ; sundial, ii, 225

Edworth, Steph. de, ii, 263

Efelmus, ii, 2 I 5 ; Affa, w. of, ii, 215

Egerton, Hume (Cust), ii, $66 n$

John, see Bridgewater, carl of Sir Thos., see Ellesmere, baron Thos., see Fllesmere, earl of ; Miss, iii, 286

Eggington (Ekenden), John de, iii, $419 n$

Eggington (Ekendon), iii, 368, 399 , 400,402 ; chap., i, 341 ; ii, I I 4 ; iii, 4I4, 4I7; ch., iii, 400, 4 I ; man., iii, 408; Nonconf., iii, 415 ; vicarage, $\mathrm{iii}, 4 \mathrm{I} 4$

Eggington House, iii, 400

Eglemond, John de, iii, 448

Eglemunt's quarry, iii, 448

Eglinton, I.d., ii, I92

Egremont, Sir John, iii, 298, 299

Egton (Northants), man., i, 365

Eitona, see Eaton Bray

Ejected clergy, i, 338

Ekelston, Kath., iii, I06 ; Rob., iii, I06

Ekenden, see Eggington

Ekendon, see Eggington

Elbone, Rob., ii, I6o

Eldred, John, iii, I Io, I 75 n, 247, 253,331

Eldridge, John, iii, 327

Eleanor, queen, i, $373 n$; iii, I6 $351,353,370$; sister of Hen III., ii, 306, 350, 439

Elgin, Diana, ctss, of, iii, 3 I $4,3^{2} 5$; earls of, ii, $44,49,55 n, 267,325$ iii, 3 I 5, 324 ; Rob., ii, I47, 268 Thos., iii, 290, 3I4, 3 I6 ; Ld., iii, $27^{\circ}$
Elizabeth, queen, ii, 295, 3 ro ; iii $42,252,276,339,353,376,459$ Elkin, Mary, iii, 408

Ell, Will., ii, $94 n$

Ellenborough, Ld., ii, 65

Ellesmere, Ld., iii, IO2 ; Thos. earl of, iii, $44^{8}$

Ellingham, Dan., iii, 4I3, 4 I 4 ; Eliz. iii, 413 ; Thos., iii, 407

Elliot (Elliott), Florence, iii, I26; Rob., iii, 126

Ellis, Thos., iii, 243

Elmele, Gawin, iii, 453

Elmes, Ann, iii, I59; Edm., iii 1 58; Thos., iii, I 58, I 59 ; Will. iii, I 59

Elmore (Gloucs.), iii, 339

Elmore, - , ii, 326

Elms Farm (Goldington), iii, 202

Elnestow, see Elstow

Elrichton, Will. de, i, 402

Elstow (Elnestow), i, 120, 353, 356 ; ii, 58 , II5, I90, I97; iii, 2, 4I, 205, 226, 267, 279-84, 384 Abbey House, iii, $28 \mathrm{I}$; adv., $i$, 326 ; iii, 281,283 ; chant., $i$, 326 ; iii, 284 ; chap., iii, $283-4$ char., iii, 284 ; ch., i, 3 I $5 n, 325 n$, $326,353,354,355 n$; iif, 279 , $282-3$; crown property, iii, $27 \mathrm{I}$ : earthworks, i, 305; fairs, iii, 28 I inds, iii, 280 ; inns, iii, 280 . man., i, 313 ; iii, 280,281 ; mkt. cross, iii, 279; moot hall, iii 279; place-names, iii, 279,280 ; prebend, iii, $284 n$; rectory, iii 284 ; Rom. rem., iii, 280 ; Samian ware, ii, 7 ; sch., i, 350,355 ; ii, I 5o, I 77-8, I 82 ; vicarage, i, 3 I $7 n$, 327

Elstow, Grace, iii, 25 ; John, iii, 25

Elstow Abbey, i, 3 I3, 3I 4, 3 I 5 n $316 n, 326,332,349,353,355-8$ $362,364,383,398$; ii, 332,333 , $33^{8}, 380$; iii, $23,237,282,284$, $304,314,315,326,328,353,438$, 454 ; abbesses, i, $357,380 n$; ii, $296 n$; seal, i, 36r

Eltesdon (Filtesdone, Eltesden), John de, iii, 428, 429, 430, 449; Marg. de, iil, 428 ; Walt. de, iii, 428 ; Will. de, i, $325 n, 326 n$; iii, $428,455,432,449$

Elton, John de, ii, 280

Elvendone, iii, 123

Elwes, Dudley Cary, ii, $25 n$; Geoff. iii, 393

Elwyn, Rich., i, 399

Ely, bps. of, i, 3 I 4 n, 400 ; ii, 2 I 4 , 3 $2,332,374,380$; iii, 29, 30, г 1 , I65, 209, 2I4, 219, 304, 366; John de Kyrkeby, iii, 39

Ely, diocese, i, 347

Ely, Isle of, ii, 25, 31-32

Emerton (Emmerton), Will., ii, 292, 335; i ii, $37^{8}, 44_{4} ;-$, ii, 324 ; iii, $39,315,328,334,348$, 398,435

Emery, Eliz., ii, 289, 293 ; iii, 274 Florence, ii, 262 ; Hen., i, 363-5; Rich., ii, 28r, 289; Sarah, ii, 289, 293; iii, 274 ; Thos., ii, 262

Emmanuel College (Camb.), ii, I63, I67, I68

Enderbies man. (Sutton), ii, 248

Enderby, Alice, ii, 2 I I, 224 Eleanor, ii, 2 I I, 22 I, 224, 248 iii, 230,248 ; John, ii, 36,204 $210,2 I I, 212,214,220,224$ iii, 230: Maud, ii, 204, 2 I I, 2 I 4 224 ; iii, 230 ; Rich., ii, 204, 210 , 2 I I, 224 ; iii, 230

Enfield, Barth. de, iii, I68, 172

Engayne, Clarice, iii, 2 I I ; Eliz. d", ii, 243; iii, I9r; Joan d', iii,

\section{Engayne (cont.)}

191, r94: John, i, $325 n, 36 \mathrm{r}$, 385 ; ii, 243,245 ; iii, I9r, I94, 20I; Joyce d', ii, 243 : iii, I9I, I94; Kath., ii, 243; Mary d', ii, 243 ; iii, I9I; Nich., iii, 211 ; Sim., iii, 2 I r ; Thos. d', ii, 243 ; iii, I9r : Will,, iii, 2 I 4 ; fam., iii, 2 I0, 2 I I, 33 I

Engelar, ii, 305

Engelram, iii, $37^{\circ}$

Engineering works, ii, 125

Engleys, Will, le, iii, I62

Englysshe, Hen., iii, 220 ; Marg., iii, 220 ; Thos., iii, 220

Enveyse, see L'Enveise

Episcopacy, pet. for abolition, i, 337

Eppeho Wood, iii, 442

Erchtdehene, Isabel la, iii, I68

Erfast, ii, 263, 281, 283; iii, 309

Erneys, Edw., iii, 144; Elinor, iii I 44 ; John, iii, I44, I 47 ; Phil., iii, I 44 ; Rog., iii, I 44 ; Walt., iii, I 44 ; Will., iii, I 44

Ernuin (Ernui), i, 312 ; iii, 37, 237

Eseltone, see Shelton

Espaigne, Thos. d', ii, 227

Espec, see Spech

Esperun, Thos., ii, 253

Esseby, Dav. de, iii, 3 I 4 ; Hugh de, iii, 3I4; Rob., iii, 67

Essex, assessment, ii, $48 n$; ealdormanry, ii, I9; peasants' revolt, ii, 35 ; straw-plait. ind., ii, 12 I

Essex, Roais, ct. of, see Beauchamp, Rohesia de ; earls of, ii, 45, 46, 47, 53, 354; iii, I I I ; Geoff. 390 ; iil, 9, 70, 169, I 7 I ; Hen., il, 295: Humphrey de Bohun, ii, $223 n$; Rob., ii, 339; Will., ii, I 54 ; iii, I73

Estcotes, see Eastcotts

Estfeld (Riseley), iii, I57 $n$

Esthaddon, Ralph of, i, 402

Estodham, see Studham

Eston, Christina, ii, 212 ; John, ii, 59; iii, 8 ; Ralph de, i, 401 ; Rich., ii, 212 ; Thos., ii, 212 ; fam., ii, 55 Estons, man. (Holme), ii, 212

Estwick, Anne, iii, 4I8, 4 I9 ;

Chris., ii, 40; iii, $384,418,419$;

Marion, iii, 4 I9

Ethelfled, ii, 18

Ethelred, earl, iii, 345

Ethelred II, iii, 345,383

Eton, par. see Eaton Socon

Eton (Etone), John de, iii, 201 Laur., ii, 295 ; Nich. de, iii, I 88 ; Ulmar of, i, 300 ; ii, $271 n, 277$, 304

Etonbury, earthwork (Arlesey), i, 278-80 ; ii, 26 I

Etonbury, man. (Arlesey), ii, $223 n$, $234,261,262,263,265$; mkt., ii, 26r, 263; mill, ii, $26 \mathrm{I}$

Eton Coll. (Bucks), i, 404 ; ii, 157 ; $\mathrm{iii}, 47,48,49$, I 52, 4 or, 403

Etrick, Ant., ï, 359 ; Eliz., ii, 359

Eu, Ralph de Issoudun, earl of, ii, $223 n$

$\mathrm{E}_{\mathrm{u}}$, Alice d', ii, $223 n$; Will. d', ii, $211,223,261,263,3^{8} 3 n, 3^{8} 4$

Eudo, iii, 2 I I, 246, 248

Eugenius IV, pope, i, 352

Eureton, see Everton

Eustace, count, iii, 74, 90, 96 n, r I r; sheriff of Hunts., iii, I68, I69

Evans, Dr. Arth., i, I70, I77; Sir John, i, I 45, I 51 , I 57 , I $58 n$, I59, I6I, I62, I66, I69, I70, I $73 n$, I74, I 90, 269

Evelyn, Geo., iii, 392

Everard, Edm., iii, 423 ; Hen., iii, 248 ; fam., iii, 422

Evereshawe, see Evorsholt 


\section{A HISTORY OF BEDFORDSHIRE}

Evereshont, see Eversholt

Evereshot, see Eversholt

Everitt, Sir John, iii, 453; John, iii, 453

Eversden Parva (Cambs), i, 360

Eversend, Will, iii, 383

Eversholt, ii, 84, 85, I I 4 ; iii, 336 . $375,45^{8}, 459$; adv., iii, $376,37^{8}$; char., iii, 378 ; ch., i, $315 n$; iii, $375,377,378$; crown property, iii, 27 I man., i, 370 ; iii, 23, 375 , 376,377 ; Nonconf., iii, 375; parson, i, 323; rectory, iii, 375 ; sch., ii, I 82

Eversholt, Alice de, iii, 429 ; John de, iii, 375, 377; Miles de, iii, 375 Paulinus de, iii, 376 ; Rich. de, iii, $377,419,429$

Eversolt, see Eversholt

Everton, ii, 7I, 96, II3, 201, 226-30, $24^{2}, 255,262$; iii, 8; adv., ii, 229 ; char., ii, 229, 230 ; ch., i, 345 ; ii, 228 ; man., ii, 226, 227, 228,238 ; man.-house, ii, 226 ; mkts. and fairs, ii, 227, 228 place-names, ii, 226; sch., ii, I 82

Everton Biggin, man., ii, 228

Everton Norsbury, man., ii, 227, 228

Eveshall, Rich. de, iii, 362

Evretune, see Everton

Evreux, Almaric, ct. of, iii, 390 : Millicent, ctss. of, iii, 390

Evyesholt, see Eversholt

Ewell (Surrey), Roman well, ii, 6

Ewelme (Oxon), ii, I57

Ewen, Rich., ii, 318

Exeter, bps. of, i, $315 n$; iii, I42 Bartholomew, iii, 143 ; Hen. Phil potts, ii, 175

Exeter, Dorothy, ctss. of, iii, 235 330; Anne, dchss. of, jii, I02 dks. of, iii, 272 ; Hen., iii, 102 272 ; earls of, iii, 13, I5; iii, 236 ; Brownlow, iii, I4; David, iii, I4, 235 ; John, iii, I4, IOI, IO2; Thos., iii, I 4, 235, 330 ; Will. iii, 235 ; Hen., mqs. of, iii, I4

Exeter Wood (Cardington), iii, 235

Exmore, Edw., i, 399

Exmouth, Clementia de, iii, I20 John de, iii, 120

Eye (Eya), ii, 336, 337 ; man., ii, 333

Eymewe, Thos., ii, 332

Eyr, Le, Will., iii, I I6 $n$; fam., iii, I 13

Eyre, Mrs., ii, 374

Eythorne (Kent), i, 389 n

Eytona, see Eaton Bray

Eyworth (Aieworde, Aisseworde) ii, II3, 20I, 2I9, 230-33; ii I06; adv., i, $362 n$; ii, 233 ; char., ii, 233 ; ch., i, 315, 333362 ; ii 230, 231 ; field-names, ii, 230 mans., ii, 143, 230-3 ; vicarage i, 327 ; windmill, ii, 232

Eywurth, see Eyworth

Faber, Will., iii, 95

Facey, John, iii, 458

Fafiton, Rob., ii, 328

Fage, John, iii, 220, 222

Fagg, Anne, ii, 235; Sir John, ii, 235

Fairclough, John, iii, 204, 205 . Mary, iii, 204 ; Sam., i, 342

Fairfax, Sir Thos., ii, 48, 49, 50, 54 ; iii, 35, 227

Fairfield (Biggleswade), i, I Io

Fairie, see Fayrey

Fairs, ii, $263,330,349,350,35$ I $n$, $353 n$; iii, I6, I7, 21, 35, 64, 69

Falbe, Mme. de, ii, 353

Falconer's Hall, man., see Greathampstead, man.
Falconer's Hall Farm (Luton), ii, $35^{8}$

Faldo, ii, 345 ; man., ii, 346

Faldo (Faldoe, Faldho), Agnes, iii, 39; Amphyllis, iii, 315; Anne, iii, 3I5; Cecilia, ii, 346; John ii, $164 n$; iii, 39 ; Paul, iii, 27 ; Rich., iii, I5I. 315 ; Rob., ii, I64, 357 ; iii, 3I5, 351 ; Thos., iii, I 8 ; Will. (de), ii, $346,377,379$; iii, $39, I_{4} 6,315$

Faldo Farm (Faldo), ii, 344, 346

Falkland, Lucius Chas., vsct., iii, 332 ; Sarah, vsctss., iii, 332

Fancott (Toddington), iii, 439; almshouses, iii, 439

Fane, Mrs. V., iii, 23I, 233; Will., iii, $368 ;-$, iii, 23 I

Fanhope, Geoff., baron, iii, 31 7 : John, Ld., iii, 272, 285 ; see also Cornwall, Sir John

Fanshawe, Fred., ii, I74, I75; Kath., ii, 3I7 ; Sir Thos., ii, 3I 7 ; Thos., ii, 3 I 7

Farele, WVill., iii, $294 n$

Farleigh (Hants), iii, 405

Farley, ii, 342 ; hermitage, i, 400 ; hosps., i, 349, 400 ; ii, 357 ; man., ii, $350,357,35^{8}, 360$, $361 n$

Farmer, Anne, Laさy, iii, 452 ; Eliz., Lady, iii, $45^{2}$; Sir Geo., iii, $45^{2}$; Sir Halton, iii, 452 ; Joanna, iii, I15; Mary, Lady, iii, 452, 454 ; Rich., ii, 44; Sir Will., iii, 452 ; Will., Ld. Leominster, see Leominster

Farm House (Eaton Socon), iii, 193

Farndish, ii, II 5 ; iii, 34, 57 ; adv. iii, 58 ; bronze ornaments, i, I 89 , I90; char., iii, 58 ; ch., iii, 58 ; man., iii, 57,; Manor Farm., iii, 57; Rectory Farm, iii, 57

Farndissche, see Farndish

Farnedis, see Farndish

Farnham, John, iii, 23

Farrer (Farrar), Anne, iii, 66 Rev. C. F., ii, I78; Denis, iii, I $87,281,304$; Eliz., iii, 281, 304 Eliz., iii, 304 ; Farrer G. S., iii, 304 ; Frances, iii, 304 ; Fran, ii, 40 ; iii, 66; Geo., iii, 68; Helen, iii, 66; Jenny, iii, 304 ; Mary, iii, 37, 66; Ralph, iii, 66; Thos., iii, 66 ; Will., ii, I68; iii, 20, 37, 66, 68, 304; fam., ii, 59 ; see also Ferrers

Farwell, John, ii, 262

Faryndysh, see Farndish

Fatt, Mrs., ii, 3I 4

Fauconer, Thos., ii, 295, 3 10

Fawcett, John, ii, 335

Fawkeswell, Marg., i, $360 n$

Fayrey, Agnes, iii, 367 ; Hen., ii, 40; iii, 367 ; John, iii, 367 ; Mary, iii, 367 ; Thos., iii, I 33

Faysey pall, iii, 367

Fécamp, Aubrée de, i, 357

Feery, Mary, ii, 256

Feevell, Will., jii, 244

Feilde, see Field

Feke, Jas., iii, 300

Felbrigge, Sim., ii, 382

Feldemerishull (Studham), iii, 426, 428

Fell, Dr., iii, 275

Felmersham (Flammersham), i, 324 ; ii, II 5, I 38 ; iii, $34,54,59-63$ 68, 73; adv., iii, 63; bridge, iii, 59 ; char., iii, 63 ; ch., i, 3 I $5 n$ 318,326 ; iii, $59,61,80$ deanery, $i, 347$; inclosure of land, ii, 97, 98; man., iii, 60; mill, iii, 59 ; sch., ii, 182 ; vicarage, i, $326 n, 327$
Felmersham, John (of), i, 400 ; iii, I 73

Felmersham Hardwick, iii, 59

Felmersham Mead, ii, 92

Fenlake, i, 380; iii, 227, 234： prehist. rem., i, I45, iii, 234

Fenlake Barns (Cardington Priors, Regine), man., iii, 236

Fenlake Mead, ii, 92

Fennels Grove, man., ii, $350,352 n$, $35^{8}$; mill, ii, 358,366

Fenner, Joan, iii, 392

Fenny Stratford (Bucks), ii, $3 n$ $71 ;$ iii, 383,402

Fens, the, ii, $61 n$

Fenton (Fentoun), Thos., vsct., ii, $267,310,322$; iii, 27 I, 331

Fenwick, Rev. Chas., jii, 304

Feoffee (Charity) Estate (Ampthill), iii, 275

Feoffee Farm (Fancott), iii, 439

Fermband, Alice, iii, 384 ; Amabilia, iii, 386 ; Eliz., iii, $343 n$; John, iii, 36, 386 ; Kath., iii, 386 ; Nich., iii, $36,46,343,386,387$; Thos., iii, $343,384,386$

Fermor (Fermour), Jer., ii, 355 Rich., ii, 329, 355, 357, 359, $378 n$

Fernadis, see Farndish

Fernedis, see Farndish

Ferneslie (Verneslie), -, iii, 244

Ferns, i, 55-6

Farrell, Rev. Maur., iii, 238, 313; Will., iii, 28

Ferrers (Ferrars), Agatha de, ii, 35I ; Anne, ii, 23I, 3 I 7 ; Edw., ii, 212, 237, 297; Eleanor, ii, 35I ; Eliz., ii, 3I6; Geo., ii, 3I6, 3I7, 3I 8; Hen., see Groby, Ld. de ; Isabel de, ii, 35I ; Joan de, ii, $35 \mathrm{I}$; iii, 452 ; Sir John, ii, 3I 7 ; Julius, ii, 3I 7 ; Kath., ii, 317 ; Knighton, ii, 3I 7 ; Maud de, ii, 35I': Rob. (de), iii, 427, 452; Sybil de, ii, 35I; Thos., ii, 3I6; Will. de, iii, 212 ; see also Derby, earl of, and Groby, Ld. de ; fam., iii, I 39 ; see also Farrer

Fettiplace, Alex., ii, 253; Anne, ii, 207, 253; Sir Edm., ii, 253; Edm., ii, 207; John, ii, 207; Will., ii, 207; -, ii, 44

ffitche, John L., ii, 268 ; see also Fitch

Field (Feilde), Anne, ii ${ }_{1} 34^{2}$; Chas. ii, 69 ; Sir Chas. V., ji, 268; Eliz., iii, 278 ; Geo., ii, 361 ; Henrietta, ii, 268 ; Hen., jii, 421 ; Jas., ii, 36r ; John, ii, 268; Susan, ii, 216 ; Thos., ii, 342, $36 \mathrm{I}$; iii, $37 n$; Will., ii, 304 ; iii, 277

Fielden, Hannah, ii, $35^{8}$; Rich., ii, $35^{8}$

Fielder, R., iii, 273

Fields-Clarke, G. T., ii, I 47 n

Fiennes, Edw., see Clynton, Id.

Finaunce (Fynaunce), Agnes, iii, 97, 396 ; Jas., iii, 97 ; John, iii, 97, 396

Finch, Rich, iii, 367

Finderne, see Fynderne

Finlinson, A. W., ii, 200

Fiott, John, iii, 92

Firegod, Rob., iii, 76

Fish (Fishe), Sir Edw., iii, 260 : Geo., iii, 227, 246, 259, 260: Humph., iii, 245, 26r ; Sir John, iii, 260 ; John, ii, 28I, 285, $342 n$; Marg., iii, 245

Fisher (Fyssher), Agnes, Lady, iii, 257 ; Agnes, ii, 277, 323; iii, 4I, 194, 396 ; Ann, iii, 286; Geo., ii, 322,323 ; Hen., ii, 322, 323 ; 


\section{INDEX}

Fisher (cont.)

Jasper, iii, 226; Jeffrey, iii, 6r, 286 ; Sir John, ii, 276, 277, 28o, 323; iii, 194, 257 ; John, i, $365 n$; ii, 277, 279; iii, 50, 1 30, 194, 259, 299, 392 ; Sir Mich., ii, 277, 278, 279 ; iii, 4I, 61, 257, 259, 305, 396: Mich., ii, 323; iii, I54, I94; Nath., iii, 43I ; Rich., iii, 208 ; Susan, iii, I56; Thos., i, 399 ; ii, 165 ; iii, $\mathbf{r}_{4} 6$

Fisheries, ii, 212, 323, 351, 366 ; iii, II, $38,44 n, 47,55,59,60$, $65,66,73,74,78,79,91$, 102, I I $4, I_{25}, I_{29}$, I $_{30}, I_{43}, 1_{44}, 1_{4} 6$, I 47, 149, 184, 196, 199, 244, 259, $299,301,387,453$

Fisher's Grange (Southall), iii, 257 Fishes, i, 98-ror

Fishponds, ii, 288; iii, 205, 28o, $343,354 n, 370 n, 372,406$

Fitch, Rev. S. E., i, 176, I 77 ; see also ffitch

Fitz, Alice, ii, 329 ; Eliz., ii, 329 ; Geo., ii, 378, 381 ; Joan, ii, 329, 330; John, iii, 231 ; Sim., ii, $329,377,378,384$; Susan, ii, 329, 378 ; Will., ii, 329,377 ; iii, 249,250

Fitzakerly, John, ii, 303

Fitz Brian, Thos., iii, $176 n$

Fitz Geoffrey (Fitz Geffrey, Fitz Jeffery, Fitz Jeffry), Edw., iii, 146; Elinor, iii, I 44 ; Eliz., iii, I05, I44, I 46, I 83 ; Fran., iii, I29, 132, I84, 220 ; Geo., iii, I05, I46, 183, 212, 214 ; Gerard, iii, 213; Jane, iii, 183 ; Joan, iii, I83, 422 ; John, iii, 105, $106 n$, I 29, 146, 147, I83, I85, 220 ; Judith, iij, I46, 183 ; Leonard, iii, I29; Will., iii, 105, 146, 183, 2 I 2 ; - W. of John, iii, I 29

Fitzgerald, Rev. W. G., iii, 29

Fitz Herbert, Edm., ii, $35^{2} ;$ Reg. ii, $35 \mathrm{I}$

Fitz Hugh, Anne, iii, I93; Joan, iii, 106; John, ii, 383; iii, 106 ; Mary, iii, 193; Nich., iii, I95; Rich., iii, 196, 197, 282; Rob., jii, I93; Thos., ii, 329, 330 ; iii, r93 ; Will., iii, I 96

Fitz James, Rich., i, 399

Fitz Jeffery, Fitz Jeffry, see Fitz Geoffrey

Fitzjeffry's, man., see Clapham Greenacres

Fitz Jocelin, Nich., iii, 133

Fitz Lewis, Eleanor, iii, 115

Fitz Neel, Grace, ii, 358 ; Rob., ii, 358

Fitz Otho, Beatrice, iii, 12, 13, 46, 330 ; Maud, iii, 13, 46, $33^{\circ}$; Thos., iii, 12, 13,46

Fitz Patrick, John, see Upper Ossory, earl of ; Mary, see Fox; Rich., ii, 6r ; W. E., iii, 228 ; W. L., ii, I 89

Fitz Payn (Fitz Payne), Ela, iii, 426 ; Rob., iii, 426

Fitz Peter, Sim., i, 378 ; ii, 26

Fitz Piers (Peter), Geoff., ii, r 44 ; see also Essex, earl of; Hen., ii, 306; Joan, ii, 35I ; Reg., ii, 351 ; -, w. of Geoff., $i, 391$

Fitz Ralph, Rich., iii, 2 I

Fitz Richard, Alice, ii, 345 ; Emma, ii, $329 n$; Gilb., iii, I $42 n$; Hugh, ii, 326 ; Ralph, ii, $329,330,332$,

Fitz Robert, Rob., iii, I 45

Fitzroy, Anne, ii, 379 ; Geo., ii, $37^{8} n, 379$

Fitz Salomon, Gilb., i, 296 n

Fitz Walter, Rob., ii, 361, 362
Fitz Warin (Fitz Warine), Agnes, iii, 70; Fulk, ii, 29; Hugh, ii, 362 ; Pet., ii, 362 ; Will., ii, 362 ? iii, $69,70,74$

Fitzwilliam, earl, iii, 3r ; Ld., ii, $61 ; \mathrm{iii}, 272$

Fitz William, Alice, iii, 300 ; Eliz., ii, 323 ; iii, 192, 244 ; Fran., iii, 300 ; Geoff., ii, I 44 ; Humph., ii, 40 ; iii, 300,398 ; Jane, Iady, iii, 3oo; Joan, Lady, ii, 323,325 ; iii, 244,250 ; Sir John, iii, 300 : John, iii, I62, I92 ; Kath., ii, 323 ; iii, 244 ; Mabel, ii, 32.3 ; iii, 244 ; Ralph, iii, I9r $n, 192$ Rob., iii, I92 ; Thos., iii, 300, 305; Sir Will., ii, 323, 325 ; iii, $244,250,25$ I, 300 ; Will., ii, 252 ; iii, 300 ; fam., iii, 303

Fitz Wirnart, Sim., ii, I 45

Five Knolls (Dunstable Downs) prehist. rem., i, 169, 270; iii, 448 ; Rom. rem., ii, 3

Flamstead (Herts), ii, 31 4, 375

Flamvill (Flamville), Alice, iii, 293 ; Hamo, iii, 216 ; Hen., iii, 206 , 216, 293; Jas., iii, 216; John, iii, 216, 293: Matilda, iii, 293 Rob. de, ii, 34I ; iii, 293, 2 I6; fam., iii, 216

Flamwelles (Flamwells, Flavells), man., iii, 216, 291, 293

Flannel manufactory, iii, 4

Flaun, Ad., i, 325

Flavells, man., see Flamwelles, man.

Fleet, riv., see Flitt

Fleet, Ralph, i, $3^{6} 4 n$; w. of Ralph, i, $364 n$

Fleete, deanery, i, 347, 35

Fleetwood, Gen. Chas., iii, I 25 Hen., iji, 104; Smith, iii, I25: Sir Will, iii, I25, 456; - ii, $5 \mathrm{I} ; \mathrm{iii}, \mathrm{I} 25$

Fleming, Hugh le, iii, 82, 84,92 , I04; Saer, ii, 234 ; iii, 70 : Sim. ii, 234; iii, 70 ; Sybil le, ii, 235 i iii, 70 ; Walt., ii, 143, 204, 205 $210 \mathrm{n}, 21 \mathrm{I}, 234,235,282-3,309$ 326 ; iii, 69,70,73,82, 92, 104 II 3, I I $8,119,146,260$

Flemish colony, iii, 268

Fletcher, Will., ii, 204; W., ii, I99

Flewton End (Milton Ernest), iii,

$\mathrm{I} 44$
Flichtam, see Flitton-cum-Silsoe

Flietewicke, see Flitwick

Flint implements, i, 269 ; ii, 5

Flints, Thos., iii, $429 n$

Flit, riv., see Flitt

Flitcroft, Hen., iii, 459, 460

Flitt, hund., ii, II $3 n, I_{4}, 26$ I, $293,306-87$, bailiff of, ii, 383

Flitt (Flit, Fleet), riv., i, 286 ; ii I 38,320 ; iii, 284, 286, 313,438

Flitton, see Flitton-cum-Silsoe

Flitton, Pct. de, ii, 328 ; Phil. de, ii, 328 ; Will. of, $i, 366$

Flitton-cum-Silsoe, i, $174,325 n$ 352,357 ; ii, $36,114,139,283$ $290,305-6,325-34,336,376,378$, 15I; iii, 316; adv., ii, 332 chap., i, 3I5n: char., ii, 333 ch., i, 3I4 $n, 315 n, 318,357$; ii, 36 , 331 ; crops, ii, 326 ; Lock-Up tower, ii, 326; man., ii, 328 mausoleum, ii, 327 ; minister' stipend, i, 34I ; Rom. burials ii, I, 7 ; vicarage, i, $316 n$; ii, 326 Flitton Greenfield, ii, $\mathrm{r} 82$

Flitton Moor, ii, 326

Flitwick, i, I45, 286; ii, 85, I15, I35, I 38, I 39, I 45: iii, 6I, 267 $274,284-88,435$; adv., iii, 288 chalybeate springs, ii, II 8 ; iij,
Flitwick (cont.)

284; char., ii, 214 ; iii, 288 . ch., i, $286,326,371 n$; iii, 287,347 ; coins, ii, 2 ; crown property in, iii, $27 \mathrm{r}$; inclosures, ii, 97 ; ind., ii, II 7 ; irrigation, ii, 135; man., i, 377 ; ii, 197 : iii, 284-6; man.-house, iii, 284 mill, iii, 286-7; Nonconf., iii, 284 ; place-names, iii, 284 ; Rom. rem., ii, 7 ; sch., ii, 182

Flitwick, Ad. de, iii, 285; Amabel de, iii, 285 ; Barth. de, ii, 378 . iii, 395; Dav. de, ii, $37^{8}$; iii, $285,395,398$; Dav. R. de, iii, 285 ; Eleanor de, iii, 285 ; Joan de, iii, 285

Flitwick Moor, ii, I18, 326 ; iii, 284 ; Rom. implements, ii, 7

Flitwick Park, ii, I 46

Flitwick Plantation (Steppingley), iii, 324

Flower, A. D., ii, 200

Flower-culture ii, I39; iii, 360

Floyer, Pet., iii, 104; - , ii, 357

Flythewick, see Flitwick

Foliot (Foliott), Alice, ii, 346; Margery, ii, 247 ; Mary, iii, Io6: Rog. ii, 346 ; Rich., i, $385-6$; ii, 247 ; iii, I06; Rob., ii, 247 ; iii, 70

Folly Pit (Caddington), i, I 5 I

Fontaine, see Fountain

Fontevraud Abbey, see Fontevrault Fontevrault Abbey (France), i, $319 n, 370,402-4$; iii, 370, 401-4 408,429

Fonts, ii, 209, 213 3 2 I 8, 222, 225, 229 $24 \mathrm{I}, 245,250,254,258,265,269$ 279, 285, 292, 303, 312, 319, 338, $347,372,380,383,384,387$; iii, $25,26,27,28,39,43,53,56,58$, $63,68,75,80,87,94,99,103$, I08, I I 6, I $21,127,131,135$, I 38 , I 4 I, I48, I 52, I56, 160, I64, I 67 , I $70, I 78, I 84, I 88,200,208,2$ I 4 $2 \mathrm{I} 7,2 \mathrm{I} 8,222,226,233,237,254$, $26 \mathrm{I}, 265,273,275,283,288,294$, $303,312,323,333,340,345,347$ $365,374,378,382,385,387,393$, $398,4^{\mathrm{IO}}, 4^{\mathrm{II}}, 4^{\mathrm{I} 2}, 4^{\mathrm{I}} 3,4^{20}, 4^{2} 3$, $434,43^{8}, 445,450,454$

Ford (Fort), ii, I 53, I 54 ; iii, 198

Forde, Joln atte, iii, 376

Ford End (Biddenham), see Kirtons Ford End (Eaton Socon), iii, I 9 I

Forder, B. J., \& Sons, iii, 329

Fordham, Geo., ii, I94 ; Rupert, iii, 256

Foreigners, fines for, iii, 17

Forester, see Forster and Foster

Forestry, ii, I 43-8

Forrest, friar, i, 33r $n, 369$

Forster (Forester), Diana, ii, 286 ; Eliz, ii, 330 ; Giles, i, 334 ; Thos. ii, 330 ; see also Foster

Fort, Samson le, i, $387,388,389$; iii, 65; Will. 1c, see Albemarle, carl of

Fortescue, Alice, iii, II2; Fran., ii, 23I; Hen., ii, 231 ; John, ii, 231 ; iii, II 2 ; Mrs., i, 335 n

Forty Foot Lane (Podington), iii, 80

Foscall, Thos., iii, 293

Fossey, F. J., i, r69; fam., ii, 206

Fossils, i, 6-12, 15, 16, 18-21, 23, 26-8, 32-4, I 50, 169 ; iii, 44

Foster, Chas., iii, 236 ; Edw., J. F. iii, 335 ; Eliz., iii, 236 ; Gabriel, iii, 434 ; Geo., iii, 287 ; Hen., iii, 236; John, iii, 236, 311 ; J., ii, I34, I35, I39; J. N., iii, 335 Martha, iii, 236 ; Mary, ii, $20_{4} n$; iii, 236; Rebecca, iii, 236; Rog. ii, $204 n$; Thos., iii, 195, 238 ; 


\section{A HISTORY OF BEDFORDSHIRE}

Foster (cont.)

Dr. Will., ii, 59, I67;-, ii, 97 , I 31, I33; iii, 195, 329; fam., ii, 243 ; see also Forster

Foster's Hill, iii, 5

Fotherby, Dr., iii, 22

Fotheringay, Agnes de, iii, $110 n$; Thos, de, iii, Iro $n$

Fotheringhay, college, ii, 235

Foucault, Daniel, iii, 2 I 2

Foule, John, ii, 296

Foulislea, (Ámpthill), iii, 268

Foullerton, Dav., ii, 320; see also Fullerton

Fountain (Fontaine), Sir Erasmus, ii, I80, 21 2, 235; John, ii, $235 n$; iii, 462 ; Kath., iii, 307 ; Rob., iii, 307

Fountains, Rog. of, i, $37^{\circ}$

Fountains Abbey (Yorks), i, 366 , $367 n$

Fourneys, John de, ii, 220

Fowler, Edw., iii, 433; Fran., ii 285 ; Gabriel, iii, 433,435 ; $\mathrm{Dr}$. G. H., iii, 338; Sir Rich., iii, 433; Rich., iii, 406, 434 ; Sybil, iii, 433; Villiers, ii, 344 ; Mrs. Villiers, iii, 296 ; Dr., i, $342 n$

Fox, Geo., i, 34I, 343, 345; Georgina, iii, 320; John, iii, 136 ; Mary, ii, 61 ; ii, I36; Rich., iii, I58, I67; Steph., ii, 6r ; iii

Fox Burrow (Foxborough Hill, Toddington), i, I 85; Rom. rem., ii, 14

Frambolt (Frampolt), Godwin, iii 52,83 , I 19

Franchenat, Isabel, iii, 347 ; Sim. iii, 347

Franchevaler, Sim., iii, 442

Francis, Ad., ii, 23i ; Eliz., ii 231 ; Jonathan, ii, I80; Sim., ii, 286: - ii, I94; see also Fraunceys

Franciscan Friars, see Friars, Grey

Franck, see Frank

Francklin, see Franklin

Francroft Wood (Sharnbrook), iii, 89

Frank (Franck), Anne, ii, 316: Thos., ii, 316; iii, 278 ; Walt. iii, $278 n$

Franklin (Francklin, Franklyn) Dorothy, iii, 127; Edm., iii, $208 n$, 212 ; Eliz., ii, 295 ; Geo., ii, I $84 n, 266$; iii, 109, I 82, 204, $208 n, 212$; Sir John, ii, 295, iii, 182; John, ii, 69, 220, 342 , 361 ; iii, 127, I82; Rev. John, 361 ; iii, $80,126,182,208 n, 212$, 407 ; John L., iii, I26, I82 ; Jos. ii, 69; iii, 80; Lucretia, iii, 80; Nich., ii, 220; Rich., ii, 295 $36 \mathrm{I}$; iii, I82, I85; Sir Will., ii, 56; ii, 20; Will., i, 398; (parson of Haynes), ii, 343 ; fam., iii, 149

Franklins,man. (Haynes), ii, 339, 342

Fraunceys, John, i, 365 ; Will. le i, 38I; see also Francis

Fray, John, iii, I95; Kath., iji, I95

Freeman, Thos., i, 335; - ii, 69, 165

Freemonger, Humph., ii, 44

Frenches, man., see Buddenho

Frenches, Will., iii, 247

Frere, Rev. E. B., ii, 214

Frereman, Rich., iii, 22

Frere's Wood, ii, I8?

Fresshyng, Annora, ii, $243 n$; John ii, $243 n$

Freston, Alan de, i, 4 or

Friaries, i, 395-6

Friars, Black, i, 322, 323, 349, 359 $363,374 n, 395-6$
Friars, Grey, i, 322, 395; ii, 9I, 239 ; iij, 22

Friar's Grove (Goldington), iii, 208 n Friars Observants, i, 395

Friends, Society of, i, 341, 344, 345 ; iii, 9, 275, 279, 338, 4I $4,45^{8}$

Frilford (Berks), i, 178, I 88

Froghole Farm (Flitwick), iii, 288

Froissart, Will, iii, 4 I 8

Frowick, Eliz., ii, 360 ; Hen., ii, 360

Fruel, Will., iii, 92

Fruit, Will., iii, 278

Fruit-growing, ij, I39, 296

Fulbert, ij, $267 n$

Fuller, Hen., iii, 449 ; John, ii, 253 ; Will., iii, I I I ; - , iii, I I 6

Fuller's earth, i, I2, 3I ; ii, II 7 ; iii, $33^{8}$

Fullerton, John, ii, 317 ; see also Foullerton

Fulthorpe (le Dale), Thos., ii, 227, 252

Fulwood, Charlotte, ii, 289; Thos., ii, 289

Fungi, i, 6I-6

Furness, Martha, iii, 32 ; Rich., iii, 32

Furnival, Christina de, ii, 247; Gerard de, ii, 247

Furtho, Alice, ii, 224 ; Eliz., ii, 224 ; Will., ii, 224, 226

Fyler, Col., ii, 263

Fynaunce, see Finaunce

Fynderne, Alice, ii, 221 ; Mich.. ii, 221; Will, , ii, 2 I 2

Fyret, Joan de, i, $360 n$

Fysshepenny, iii, Io5

Fyssher, see Fisher

Fyson, G., ii, 198

Gadbury, Marg., ii, 233; Sir Rich,, ii, 233

Gaddesden, Gt. (Herts), iii, 429

Gaddesden, Little, iii, 426

Gaddesden, fam., see Gatesden

Gadsey Brook, iii, 2 I 4, 2 I 5

Gale, Hen., iii, I 67

Galing, fam., ii, I24

Galley Hill Camp, i, 273, 28o; ii, 9 , Io, 242

Galloway, Marg., Lady, iii, 297 ; Alan, Ld., iii, 297

Galloway, Devorgilla, iii, 297

Galoys, Joan, iii, 403; Walt., iii, 403

Galsey Wood (Bletsoe), iii, 40

Gambier, Rob., iii, 90, 33I ; Will.,

iii, 66 ; Mrs., iii, 66 ; fam., iii, 95

Gamble, John, ji, I 7 I ; Rev. J. ii, 209

Gannoch's Castle (The Gannichs), Tempsford, i, 282 ; ii, 25 I

Gaols, ii, ro3

Garden Wood (Eaton Socon), iii, 189

Gardiner, Sir Edm, ii, 59; John, iii, 258

Gardner, Dan., ii, I63, 164 ; John, iii, 408; Kirkman, iii, 428

Garnault, Anne, iii, 2 I

Garners Close (Wootton), iii, 332

Garnons, Ant., iii, I96; Christina, iii, 196

Garrard, John, ii, 377; see also Gerard

Garstin, Lt.-Col. R., ii, $7^{\circ}$

Garth, Jas., iii, 66

Garway, Hen., iii, 293: Thos., iii, 293

Gascelin, see Gastlyn

Gascoigne (Gascoyne, Gascoyn), Agnes, i, 358, 380 $n, 390$. Dorothy, iii, I 5, 235 ; Eliz., iij, 231, 235 ; Geo., ii, I67, 342 ; $\mathrm{iii}$,
Gascoigne (cont.)

231 : Humph., i, 398 ; Jas., iii, I, 235 ; Sir John, ii, $34^{2}$; iii, I $66,184,197,235-6,284$; John, ii, 166 ; ii, 235 ; Marg., ii, 342 : iii, 235 ; Sir Will., ii, 39 ; iii, 24 , 137, 166, 181, 184, 187, 197, $235,237,270 \mathrm{n}, 292$; Will., iii, 97,230

Gascon, Will. le, iii, I 55

Gastlings (Sonthill), iii, 256

Gastlyn (Gascelin), Alice, iii, 257-8 ; Edm., iii, 257; Sir Geoff., iii, 257 ; Geoff., iii, 257-8; Joan, iii, 258: John, iii, 257-8; fam., iii, 2 I

Gastlyns (Gastlynbury), man. (Southill), iii, 257, 258; mkts., iii, 258

Gate-houses, iii, 1, 4, 7, 100, 432

Gatesden (Gaddesden), Aubrey,

Lady, iii, 406; Andufus de, iii, 406 ; Joan de, iii, 406; Sir John, iii, 406; John de, iii, 406, 409; Marg. de, iii, 406 : Pet. de, iii, 406 ; Will. de, iij, 406

Gateways, ii, 364,365 ; iii, 25,84 , $279.297,351,400,439$

Gaunt, John of, ii, 248; iii, IO2

Gayer's Hill (Toddington), iii, 439

Gayhurst, ii, I 87

Gearman, John, iii, 4 I6

Gedney, John, ii, 365

Gell, Thos., iii, 9r

Gemys, man. (Stagsden), iii, 97

Gemys, Christine, iii, 97; Eleanor, iii, 97; John, iii, 97; Rob. de, iii, 97 ; Will. de, iii, 97

Geoffrey, ii, 190; abbot of St

Albans, i, 359; ii, I 78

Geology, i, $1-32,45,59,62,63$

George, Agnes, iii, 375 ; Cuth., iii, 372

Gerard (Gerhard), Ann, iii, 392 ; Judith, ii, 28r ; Justus, iii, 392 ; Will., ii, 28r

Gerey, see Gery

Gerhard, see Gerard

Germund, ii, 275

Gery, Alan, iii, I97 $n$; Anne, iii, I97 ; Chas., iii, I98; Geo., ii, 43; iii, I66; Hester, iii, I98: Rev. H. W., iii, I28; Rebecca, iij, I65, 301 ; Sir Rich., ii, 4 I ; Rich., iii, I $37,165,195$, I97-8; Thos., iii, I66 $n$, I97 $n$; Walt., ii, 22 ; Will., ii, 22, $23,43,66 n$; iii, 1 37 , I 55, I65, I66, 193, I95, 197, I98, 301 ; fam., ii, 66; iii, I94; see also Wade-Gery

Gibbard, John, iii, 94 ; L. S., iii, 91 ; Will., iii, 94 ; - , iii, 91

Gibbins, Rob., iii, I 45

Gibbs, Sam., iij, I $8 n$; Thos., ii, 55

Giffard (Gifard), Agatha, i, 354; Geo., iii, 459; Kath., ii, 283 ;

Walt., i, 354 ; ii, 2 IO $n, 220,221$, $267,269,275$; iii, 3I0, 3I4, 343, 459; see also Buckingham, earl of ; Will., ii, 283 ; fam., ii, 7 I

Gilbert, earl, ii, 350 ; iii, I96

Gilbert, Sir Hen., ii, I 40 ; Hen., ii, 262 ; Rich., iii, 80

Gilbertine order, i, 390-3

Gildesburgh, Rog. de, ii, $35^{8}$

Gilds, i, 329-3I ; ii, I 79, 2 I 4, 374 ; iii, I5-I 7, 20I, $250,268,278,347$, $360,366,367,4$ I 5

Giles, A. J., ii, 198

Gill, Eliz., ii, 252 ; Fran., ii, 252 ; Marg., ii, 2 I I

Gillingham, Rog., ii, 295, 374 ; iii, $23^{8}$

Gilman, Rebecca, ii, 257 : Rich., ii, 257 


\section{INDEX}

Gilpin, Agnes, iii, 385 ; Esther, iii, 385 ; Kath., iii, 385 ; Margery, iii, 385 ; Pet., iii, 385 ; Pet. V., iii, 346; P. P., iii, 392, 4I7; Sir Rich., ii, 70 ; Rich., iii, 346 , $348,385,392$; Rich. T., ii, $66 n$; iii, 407, 443; Rob., iii, 385 ; Col. R. T., ii, 69; Thos., iii, 348, 385 ; fam., ii, 66; iii, 386

Girtford, i, I73; ii, 20I, 242 ; coins, ii, 242 ; man., ii, 244 ; Nonconf., ii, 242,246 ; Rom. rd., ii, 4

Girton, Alice, ii, $182 n$; iii, 278 ; John, ii, 99-100

Gisby, Rich., iii, 156

Gladley, iii, 336, 408; Grange mill, iii, 409; man., i, 376; iii, 405, 409, 422

Gladley Wood (Leighton Buzzard), iii, 408

Glasgow, ii, 139

Glass, i, 184, 299 ; ii, $217,222,226$, $233,258,273,303,308,312,324$, $326,338,372$; iii, $6,25-6,47,56$, $59,68,76,80$, 108, II0, 121, I26, I 35, I $47,151,152,156,167,169$, $174,188,198,202,208,217,218$, $226,287,295,312,344,365,423$, 450 ; Rom., ii, 9, I2, I4

Glassbrook, Ann, iii, I95

Glatton (Hunts), ii, 2 I4

Glaykyn, Will., iii, 297

Gledly, see Gladley

Glen, iii, II9

Glenister, Thos., iii, 320

Glintells (Bolnhurst), iii, 126

Gloucester, bp. of, i, 342 n

Gloucester, hon., iii, $36,39,54,76$, 78,85 , I10, $157,175,1_{76}, 219$, $343,387,405,421,422$

Gloucester, Isabel,ctss. of, $\mathrm{iii}, 390 n$ : dk. of, iii, In 8 : Thos., dk. of, iii, I56, I 7I ; earls of, ii, 29, 30, 31,32 ; iii, $36 n, 176 n, 390 n$; Gilb., iii, I 76, 362, 422 ; Rich., ii, 384 ; Rob., i, 3I4, 3I5n; ii, $24,350,356,393$; Will., ii, 350 , 356 ; iii, 393

Gloncester, Hugh of, iii, 446 ; Will. of, ii, 3 io

Gobion, Agnes, i, 359, 360; Eliz. de, ii, 381 ; Hawise, ii, 345,381 . Hugh (de), ii, 30, 345, 38I ; iii, 65 ; Isabel, i, 360 ; Kath., ii, 345 , $38 \mathrm{I}$; Marg., ii, 345 ; Maud, ii, 345 ; iii, 65; Rich. (de), ï, 345 , 346,381

Godarvill, Joan de, iii, 257 ; Walt. de, iii, 257

Goddard, A. R., ii, I9 n; Joan, iii, 53,60 ; Vincent, iii, 50, 60; Will., iii, 50,60

Goddington (Oxon), ch. i, 357

Goderich, Hen. Grey, vsct., see Kent, dk, of

Goderiche, Eleanor, iii, 285 ; John, iii, 285 ; Rob., iii, 285 ; Will., iii, 285

Godfrey, ii, 341 i iii, 133, 159, 278

Godfrey, Eliz., iii, 243, 246 : Frances, ii, 378 ; Hen., ii, 24I, 289 ; iii, $243,244,246$; Jer. iii, 328 ; Joan, ii, 289; iii, 243 ; John, ii, 378 ; iii, 29, 198 ; Jos., iii, 9I ; Marg., i, 358; Mary, iii, 198; Nich., iii, 211 ; Rebecca, iii, 9r ; Rich., ii, 204, 289; iii, 243-4

Godmar, Mary, iii, 346 ; Walt., iii, 346

Godric, iii, 133

Godwer, Rich., iii, $45 \mathrm{I}$

Godwin (Godwine), iii, 78, 1 50, 248

Godwin, Will., ii, 248; Mrs., ii, 333 ; see also Goodwin
Goff, John, iii, 68

Gold Close (Pulloxhill), ii, 376

Goldentone, see Goldington

Golderon, iii, 5 I

Goldington, i, 296, $297 n, 324,365$, 380 ; ii, $94 x, I_{1} 3, I_{54}, 199,200$ iii, 3I, 34, I09, I80, 202-09 adv., iii, 208: Bury Farm, iii, 205; char., iii, 209; ch., i, $315 n, 380$; iii, 202, 207 ; inns, iii, 202; man., iii, 203-6 mills, i, 296, $297 n$; iii, 206-7 Nonconf., iii, 202 ; place-names, iii, 202 ; sch., ii, 182 ; sheep, ii, I 30 ; vicarage, $i, 316 n, 34$ I iii, 202, 205, 208

Goldington, man. (Eaton Socon), iii, I9I, 194

Goldington, Christiana de, iii, 205 Edwin de, iii, 206; Joan de, iii, 205; John (de), ii, 243; iii, Igr, $194,272,306,422$; Joyce de, ii, 243 ; iii, 194; Mich. of, i, $38 \mathrm{r}$. Ralph de, iii, 205 ; Rog. (de), iii 205, 206; Thos. de, iii, 206, 306 Wigain de, iii, 206; Will. (de) iii, 205-6, 306, 307

Goldington Bury, iii, 202 ; man., iii, 204, 205

Goldington Grange, iii, 202

Goldington Hall, iii, 202

Goldinton, see Goldington

Gold mine (Pulloxhill), i, 31 ; ii, 376

Goldsmith, Dan., ii, 270; Eliz., iii, 444; John, i, 398 ; Mary, iii, 444; Oliver, iii, 19; Rob., ii, 224 ; Thos., iii, 444; Will., ii, 382 ; iii, 259,444

Goldstone (Goldston), Geoff., iii, 98 ; John, iii, 98 ; Pet., iii, 98 ; Rich., iii, 98 ; Thos., ii, 376 ; iii, 93

Goldyngton, see Groldington

Gonville and Caius Coll. (Camb), iii, 453

Good, Rich., ii, I57

Goodfellow, John, iii, I33

Goodman, Jas., iii, 275, 279 ; John, iii, 200

Goodwich Farm (Eaton Socon), iii, I90, 196

Goodwich, man., see Begwary

Goodwin, Edw., iii, I83; Florence, ii, 262 ; Geo., iii, 408; Hen., ii, 262; Thos., iii, 408; see also Godwin

Gooseberry-bush Hill (Luton), i, 173

Goosey, John, iii, 84, 119; J. G., iii, II9; Will., iii, 87, I22; W. G., jii, II

Goosey Lodge (Wymington), iii, I I 7

Gore, Thos., ii, 63 ; - iii, 20

Gorerong Woods (Podington), iii, 80

Gorges, Edw., Ld., iii, 83

Gorges, Sir Rob., iii, 83

Gorham, Geoff. of, iii, $350,356 n$

Gormanston, Chris., vsct., ii, 323 ;

iii, 244 ; Kath., vsctss., ii, 323 . iii, 244

Gosfield (Essex), iii, 266

Gosling End Green (Harlington), iii, 381

Gossard's Green (Cranfield), iii, 275 Gostwick, Alice, i, $335 n$; Anne, iii, 21, 263, 266, 302; Dorothy, iii, $25^{8}$; Chas., iii, 266; Sir Edw., iii, 203, 240, 264, 266; Edw., iii, $215,258,259$; Eliz., iii, 203 : Geo, iji, 240; Jane, iii, 203 : Joan, Lady, iii, 247, 266: Joan, iii, I5, 22, 203, 204, 210,212 , 214,296 ; Sir John, i, 33 I $n, 364$; iii, $215,216,240,247,262,263$, 264,266 ; John, i, 365 : ii, 219 , $220,221,266,267$; iii, 15, 22,
Gostwick (cont.)

$37,38,184,203,204,206,207$, $210,212,214,225,237,263,264$ 296: Marg., iii, 203: Rob., iii, $253,263,266$; Sir Will., ii, 59 $60 n$; iii, 203, $210,211,239,240$ $263,265,266,3$ II ; Will., ii, 2 I9, 220 ; iii, I3, I5, 2I, 203, 204, 212 $216,253,258,263,264,266,302$ $3 I I$; fam., ii, 60 ; iii, 212, 2 I 3

Goswell End (Harlington), iii, 379

Goswood, John, ii, 333

Goude, Hen. C., iii, 368

Gould, T. C., i, 281 ; - i, 306

Goushill, Eliz., ii, 340; Hadwisa de, ii, 355 ; Rich. de, ii, $231 n$; Sir Rob., ii, $34^{\circ}$

Gower, Ld., ii, rg2

Gowran, Lady, ii, 61 ; iii, 272

Goyes (Goyz), Andr. le, ii, 220 John le, ii, 220 ; Thos. le, ii, 220 ; Will. de, iii, 299

Grace, John, i, $367 n$

Grafton, Alex., ii, 127

Graham, Rich., iii, 32; W. B. 1,9 I

Graie, see Gray and Grey

Graies Bury (Henlow), ii, 28I n, 282 Grandim, Hugh, ii, 294

Grandison, Geo. V., vsct., ii, 347 : John, vsct., ii, 347: Frances, vsctss., ii, 347 ; Mary, vsctss., ii, 347

Grange, Rich., iii, 278

Grange Farm (Colesden), iii, 2 I9

Grange Farm-house (Haynes), ii, 342

Grantesden, Sim. of, i, 387

Grantham, Mary, baroness, ii, 332 Ld., ii, 327, 329

Grantmesnil (Grentmeisnil, Grantmaisnil), Adeliza, iii, 230, 231 Hugh de, ii, 2 I $n$; iii, 144, 230 293; Parnel, iii, 293

Granville, ctss., ii, 6on: John, earl, ii, 34I ; Rob., earl, ii, 210, 34I iii, 293,326

Grassineau, Jas., iii, 195

Graunson, fam., iii, 43

Graunt, John le, iii, 159

Grave, La, see Grovebury Priory

Gravel Pit Hill (Houghton Regis), ii, 8

Gravenel, Joan, ii, 230, 232 ; John, ii, 230, 232 ; Thos. de, ii, 230

Gravenhurst, Lower, ii, 4I, II 4 306, 333-4, 336-8; adv., ii, 338 : char., ii, $33^{8}$; ch., i, 3 I $5 n$, $3^{80}$ ii, 337 ; man., ii, 334,336 ; iii, $286,418 n$; ship-money, ii, 337

Gravenhurst, Upper, ii, $76, \mathbf{I I}_{4}$ $266,306,333-6$; adv., ii, 335 ; chant., ii, 335: chap., i, 324 ; char., ii, 335 ; ch., i, $324 n$, 331 ii, 334 ; man., ii, 333 ; iii, 286 Nonconf., ii, 335 ; sch., ii, 182

Gravenhurst, Elias of, ii, 336 ; Rog. of, i, 377

Graves (Greve, Greves, Greyves) Agnes, iii, I46; Edw., iii, 340 Rich., iii, I 46 ; Thos., iii, 450 : Walt., ii, 242, 244

Gravesend, bp., see Lincoln, bps. of

Gray (Graie, Graye), Ant., ii, 257; Chris., ii, 209, 229, 250, 343 iii, 58, I3I, 2I $8,283,325,425$, 438 ; F. H., iii, 386 ; Harry, ii, 332, Sir Hen., iii, 233 H. J. S., iii, 386 ; John, iii, I22 ; Mary, Lady, iii, 233; Miles, ii, $233,254,285,303,335$; iii, 167 , $200,288,378$; Pet., iii, 32 I-2: Sibella, ii, $28 \mathrm{I}$; Walt., ii, $28 \mathrm{I}$; -, Mrs., iii, 386 ; see also Grey 


\section{A HISTORY OF BEDFORDSHIRE}

Graynger, Marg., i, 393

Grazehill End (Ravensden), iii, 209 Great Englands field (Dunstable), iii, 350

Greathampstead, man. (Luton), ii, $350,352 n, 358$

Greathampstead Farm (Luton), ii, $35^{8}$

Greathampstead Someries, man. (Luton), ii, 358, 364

Great Hayes Close (Wootton), iii, 332

Green (Greene, Grene), Andr. P., iii, 211 ; Edm., ii, 156, 160, 162 . Edw., ii, 317 ; Edw. W. S., iii, 2 I ; Eliz., iii, 462 ; Fran., iii, I9, 28 ; Sir Hen., iii, 443; Hen., ii, $316 n ;$ iii, 92 ; H. H., ii, 138 ; John, ii, 266; iii, 92, 443 ; Jos., iii, I94; J. R., ii, I8 $n$; Kath. iii, 92 ; Sir Ralph, iii, 92 ; Ralph, iii, 92, 443 ; Rev. Rob., ii, 229 Rob., iii, 387 ; Rudd, iii, I81 Sir Thos., iii, 92 ; Thos., i, 403; iii, 92, 443

Greenacre, Isabel, iii, t29; John, iii, I 29 ; Rich., iii, 129

Greene, see Green

Grcen End (Gt. Barford), iii, 181

Green End (Little Staugliton), iii 165

Green End Farm-house (Gt. Barford), iii, $18 \mathrm{I}$

Greene's Closes (MauIden), iii, 275

Greenfield (Grenficld), ii, 139,326 , 376 ; man., i, 370 ; ii, 376 . mill, ii, 376 ; Nonconf., ii, 333

Grcenfield (Grencfield), Sir Kich., iii, 340 ; Thos., i, 402 ; W. B., ii, $338 ;-$, ii, 197

Greenlane, John, iii, 248

Greensbury (Grymsbury) Farm (Bolnhurst), iii, 124

Green Way (Dunstable), iii, 350

Greenway, Rich., iii, 36 I

Gregory, pope, ii, 29 ; iii, 278

Gregory, John, ii, 4I ; Rich., iii, 257

Grendon, Rob., iii, 126; Kog., iii, I26: Thos., iii, I26; Walt., iii, 126

Grene, see Green

Grenfield (Grenefield), see Greenfield

Grentmeisnil, see Grantmesnil

Grenville, Sir Bern., ii, 339; Sir Bevil, ii, 339: Sir John, ii, 339 Sir Rich., ii, 338

Gresham, Will. R., ii, 271

Gresley, Frances, Lady, iii, 282 Hawise de, iii, 339 ; Joan de, iii, 339 ; Rob. de, iil, 339 ; Sir Thos. iii, 282

Gretton (Northants), iii, 57

Greve, Greves, see Graves

Grey, Chas., earl, ii, $64 n$; Ladies, ii, 380 ; Amabel, ii, 327 ; see also Grey of Wrest, ctss.; Eliz., ii, 267: Susanna, iii, $228 n$ : Lds. de, ii, I30, 231 i ; Hen., iii, 144 Reg., i1, 283; Rich., ii, 267 ; Jemima, mchnss. de, ii, 327 332 ; Hen., mqs. de, see Kent, duke of

Grey (of [de] Groby), Lds., ii, 44 ; iii, 248 ; Hen., ii, 231 ; Will., iii, 230

Grey (de Rougemont), Thos., Ld., ii, 37

Grey (of [de] Ruthyn), Lds., i, 352 ; ii, $35,36,147,334$; iii, 65 ; Edm., ii, 37, 327 ; Edw,, ii, 323 ; Reg., ii, 327, 331; iii, 13 ; Rog. ii, 327

Grey (of [de] Wilton), Lds. i, 330 iii, 377 ; Arth., iii, 253 ; Hen., ii, 327 ; John, ii, 328 ; Reg., ii, 327
Grey (of Wrest), Amabel, ctss. de, ii, $61,323,327,332,377,380$; earls de, iii, 52; Thos. P., ii, 325,332

Grey, Agnes de, ii, 326; iii, 105; Amabel de, ii, 332; Anne (de), ii, 290, 332, 334; iii, 300 ; Anne S. de, ii, 332; Ant., ii, 219 ; Cecilia, iii, 89; Chas., ij, 290, 332; Sir Edm., ii, 215; iii, 272 ; see also Kent, earl of Eleanor, ii, 208, 283, 328 ; Eliz., iii, 78,300 ; Florence de, ii, 328; Fran. T. de, ii, 327 ; Henrietta de, ii, 332 ; Sir Hen., ii, $290,305,327,329,333,334$; iii, $82,272,286,310$; Hen. (de), ii, $85,263,290,327,332,335$; iii, $64,147,443$; see also Dorset, mqs. of ; Humph., iii, 443; Jas., iii, 444, 446; Jane, iii, 443; Jemima de, ii, 332; Sir John de, i, $325 n, 326,388,390 n$; John (de), ii, 3o, $256 n, 263$, $281 n, 283,326,327,330,332$; iii, $51,65,66,67,78,82,85,105$, $147,321,326,377,444,446$; Pet., ii, 342 ; iii, 20I, 259, 349, $367,377,443$; Sir Reg. de, ii, 33, 28I $n$; iii, 2, 360 ; Reg. de, ii, 32, 208, 263, 267, 283, $322,326,328,329,336$; iii, 5I, 7 I $n, 82,106,229,292,300,322$, 396,397 ; see also Grey (of (de) Ruthyn), Reg., Ld. ; Sir Reynold de, i, 328; Rich. (de), ii, 263 , 330 ; iii, 272 ; see also Kent, earl of; Sir Rog. de, iii, 396 ; Rog. de, ii, $146,329,331$; iii, $65,322 n$; Simeon, iii, 156; Sim., iii, 154 ; Sophia de, ii, 332; Susan, ii, 290 ; iii, I56; Thos., iii, 300 ; see also Dorset, mqs. of ; Will., iii, 444, 446; Zachery, iii, 296; -, bp., see Lincoln, bps. of ; - Lady, i, 330 ; ii, 37 ; fam., ii, $56,146,330,331,332,377$; iii, 144,321

Grey Friars, see Friars, Grey

Greystock, Eliz., baroness, iii, 192 ; John, Ld., iii, I92 ; Ralph, Ld., iii, 192

Greystock, Alice de, iii, 192 ; Ralph de, iii, 192 ; Sir Rob., iii, 192 ; Will. de, iii, 192

Greyves, see Graves

Gribble, Geo., ii, $282,283 n$; G. F., ii, 285 ; G. J., ii, 281

Griffin, Thos., iii, 307; Walt., i, $336 n$

Grigg, Alice, ii, 449 ; Geo., ii, 383, 449 ; Mich., ii, 43 ; iii, 315 ; Rich., ii, 383 ; Rob., iii, 425; Thos., iii, 3 II, 425

Grimbald, iii, 395

Grimbaud, see Grymbaud

Grimscote, man., i, 376

Grindall, archbp., ii, 159

Grivell, John, ii, 224

Grocers' Company (Londón), i, 329 ; iii, 40,49

Groome, John, iii, 385 ; Will., iii, 385

Groos, Oliver, iii, 424

Gros, Will, iii, 2 I9

Grossetête, bp., see Lincoln, bps. of

Grosvenor, earl, ii, 192

Grove, man., see Britens

Grovebury (La Grave, La Grove), man., iii, 404; farm, $21,7 \bar{i}, 80$, 82,83

Grovebury Priory (La Grave, La Grove), i, $319 n, 374 n, 403$; iii, Io, $402,429,430$

Groves, man., see Britens
Grovesfield, New (Bolnhurst), iii, 128

Grubb's Wood, ii, 187

Grym, Alice, iii, 125 ; Jas., iii, 125 ; Margery, iii, 125; Rob., iii, 125

Grymbaud, Joan, iii, 220 ; Mabel, iii, 39, 219, 220 ; Nich., iii, 220 ; Rob., iii, 220 ; Will., iii, 220

Grymsbury, man. (Bolnhurst), iii, I25, 126

Grymsbury Farm, see Greensbury Grymstede, John, ii, 219 ; Kath., ii, 219 ; Rich. de, ii, 219 ; Thos. de, ii, 219

Gudmunt, iii, 125

Guines, Bald., count oI, i, 388 ; iii $47,74,101,103-4$

Guines, Rob., de, iii, IOI

Guise, Rog., iii, 385 ; see also Gyse

Gullet Wood (Turvey), iii, I Io

Gully Hill (Luton), rem., i, 172

Gullyng, Nich., iii, 7

Gunter, abbot, iii, I 79

Gurdon, Ad., ii, 3I

Gurdun, Geoff. de, iii, 133

Gurney, bar., iii, 390

Gurney (Gurnay), Hugh de, iii $370,37^{2}, 390,391$; Millicent de, iii, $370,390,391$; Thos., iii, $304,406,45^{8}$; 一, ii, 234 ; iii, 349

Gustard, Falk, iii, 205; Rob., iii. 206: Will., iii, 206

Gutch, Will., ii, 93

Guthrum, iii, 345

Gutteridge, Rich., iii, 443

Gweldene, see Yelden

Gwyn, John, i, $33^{8}$

Gwynneth, John, i, 333

Gylberd, see Gilbert

Gylys, Thos., i, 377

Gynwell, bp., see Lincoln, bps. of

Gyrth, earl, ii, 2 I $n$

Gyrwa, South, i, I90

Gyse, Anselm (de), iii, 339, 340, 342 : Beatrix dc, iii, 339; Cath., iii, 339: John (de), iii, 339, 342 Marg. de, iii, 339 : Reg. de, iii, 339 ; fam., iii, 340; see a'so Guise

Hacche, Le (Barton), ii, 309

Hacet, Ralph, iii, 343

Hache (Northill), see Hatch

Hackett (Hacket), Eliz., iii, 425 John, i, $338 n, 343$; Jobn A., i, 341 ; Nich., iii, 424 ; Rog., iij, 307 ; Thos., iii, 424, 425

Hacklete, Rich., ii, 255

Hadden, Harrict, iii, 281 ; Capt. Jas., iii, 28 I

Haddon, Will., iii, 427

Hagar, Eliz., ii, 252

Hagenes, see Haynes

Haighton, Rev. Rich., iii, 128

Hail Weston (Hunts), ii, $7 I$; iii, 222

Haine, see Haynes

Hainton (Lincs), iii, 206

Hairum, John, ii, 78

Hakeneye, Kath. de, ii, 378 ; Rob. de, ii, $37^{8}$

Haldenby, John, iii, 456 ; Margery, iii, 456

Hale, Bernard, ii, 357; Frances, ii 385; Helen, ii, 95; John, ii, 224; iii, 159; Pain, ii, 224 . Rich., ii, 224, 296; Rowland, ii, 224; Sir Will. de, ii, 309; Will, ii, $224,296,383$; Will. E. B., ii, 224

Hales, Sir Jas., iii, 456; Marg. Lady, iii, 456

Halfhide, Geo., iii, 282 ; Mary, iij, 282 


\section{INDEX}

Halford, Anne, iii, $57 ;$ Edw., iii, 57 Falfpenny, Bernard, ii, 346 ; Mary, ii, 346 ; Thos., ii, 346 , 380

Halibred, Agnes, iii, I I4; Rich., iii, II $4 n$; Will. iii, II 4

Halifax, Geo., earl of, ii, 275 ; Geo., mqss. of, iii, 427

Haliwell, fam., see Halliwell

Haliwell Priory, i, 3I $5 n, 323$

Hall, C. P., ii, I $46 n$; Edw., iii, 374 ; Rev. Edw., iii, 342 ; E., iii, 457: Ralph, ii, 266; Rob., i, 370 ; Will., iii, 284

Hallaton (Leics.), Rom. rem., ii, 12

Hall End (Maulden), iii, 3I3, 3I 4

Halliwell (Haliwell), Anne, iii, 373 Jane, ii 340 ; iii, 373 ; Rich., ii, 340,373 ; fam., iii, 373

Halsal, see Halsoll

Halsey, Hen., ii, 360 ; Will., iii, 423

Halscy's Farm (Studham), iii, 429

Halsey Wood (Sharnbrook), iii, 89

Halsoll (Halsal), Hen., iii, 183 ; Jas., ii, 254

Halstead, Rob., iii, 115

Halsted, Alice, ii, 213; Isabel, ii, 213 ; Will., ii, 213

Halton, Eliz., ii1, 97 ; John, iii, 97

Halton-super-Humber (Lincs), i, 353,357

Halyday, Clarice, iii, $22 n$; Maurice, iii, $22 n$

Halydayes (Bedford), iii, 22

Hamelyn, Eliz., ii. 346 ; Ralph, ii, 346

Hamilton, duke of, ii, 54

Hammond, John, ii, 314

Hamo, iii, 67

Hampden, vsct., ii, 60 ; iii, 96 John, vsct., iii, 45: Thos. vsct., iii, 55

Hampden, Barbara, iii, 177 ; Edm., iii, $x 77$; John, ii, $57 n$; iii, 112 177; Rich., ii, 57; Thos., iii, I 77

Hampson, Leonard, iii, 432, 456 ; Nich., iii, 277 ; Rich., ii, 252

Hampton, Thos., iii, 300

Hamsey (Sussex), ii, 163

Hancock, Matth., iii, 133; Rev. Rob., iii, 25I

Handborough (Sussex), ii, I62

Handforth (Ches.), ii, 340

Hanefeld, iii, 123

Hanger Wood (Stagsden), iii, 96

Hankin, Will., ii, 24

Hanley, Eliz., iii, 140

Hanmer, Col. H., iii, 40I, 404 ; Sir W. E., iii, 404 ; fam., iii, 4 o6, 407

Hannot, Sam., ii, 357

Hanscombe, Jas., ii, 299; Matth., ii, 40; Reg., ii, 297; Rob., ii, 297 ; Will., ii, 295; - ii, 295

Hanscombe End, ii, 297

Hanscombe Lee, ii, $295 n$

Hanslape, Rob., i, 385

Hanslope (Bucks), ii, 27, 28

Harcourt, Arabella de, iii, 23r $n$ Celia de, iii, 231 ; Hen. de, iii, 231; John de, iii, I 22 ; Rich. (de), iii, 1 20, 231 : Will. (de), iii, r2o, 231

Harding (Hardinge, Herdyng), Anne, iii, 250: Cecilia, iii, 253: Eliz., iii, 243, 301 ; Hen., iii, 30r; John, ii, 157, 315; iii, 253 ; Lewis, iii, 243: Rich., ii, 40; iii, 243,250 ; Thos., ii, 266 ; Wvill., iii, 380

Hardings Place (Harlington), iii, 380

Hardingstone (Northants), i, I $83 n$

Hardwick (Kempston), iii, 302
Hardwick (Keysoe), iii, r 36

Hardwick (Tilbrook), iii, $17 \mathrm{I}, \mathrm{I} 72$

Hardwick (Herdewyk Herdwick, Herdwyk), Pet. de, iii, I68, I69 $n$, 172; Rich. of, i, 384 ; Will, iii, 92

Hardwicke, Phil,, earl of, ii, 6r, 327,332

Hardwicke Bridge, iii, 329

Hare, i, 370 ; iii, 347

Hare, John, iii, 285 ; Marg., ii, $216 n$

Harewelle, see Harrold

Harewode, see Harrold

Harewold, see Harrold

Hargrave (Northants), i, 393, 394

Hargrave, Edw., iii, 4I5

Harington, Isabel, Lady, iii, 89 John, Ld., iii, 89 ; Rob., Ld., iii, 89; Will., Ld., iii, 89

Harington, Cecilia, iii, 89; Eliz., iii, 89

Harringworth (Northants), i, 357

Harison, see Harrison

Harker, Prof., ii, 141

Harkett, Mary, iii, 294 ; Nich., iii, 294

Harleston, Ivo de, ii, 219; Jolnn de, ii, 219

Harlew, Ad., iii, 98

Harlewin, iii, $182 n$

Harley, Edw., Ld., iii, 137

Harlington, $i, 327 n, 366,370$; ii, $30 n, 43,114,133,410$; iii, $33^{6}$ 379; adv., iii, 382 ; chants., iii, 382 ; char., iii, 382 ; ch., i, 185 $315 n, 333,371 n, 372$; iii, 381 ; crown property, iii, 27I; deer pk., iii, 381 ; man., i, 377; iii, $379-81$; mills, ii, 278 ; iii, $37-80$ 'Rising Sun' inn, ili, 379 ; sch., ii, I 82 ; vicarage, i, 327

Harlington, Dionisia de, iii, 419 John de, iii, 4r9; Rob. de, iii, 4 I9; Walt. de, iii, 4 I9

Harlington East End, iii, 38I

Harlington Grange Farm (Streatley), iii, 379

Harlington Wood, iii, 38

Harlington Wood End, iii, 379, 380 , $3^{81}$

Harman (Herman), Fran. A., iii I46; Joan, iii, 106; John, iii, 106

Harmer (Hermer), John, iii, 310 313

Harne Chapel, see Chapel Herne

Harneys, Rog., iii, 427

Harpar, see Harper

Harpeden, Sir John, ii, $227 n$

Harpenden, ii, 310

Harper, Alice, ii, I 52, I 58, 1 59, 172 Joan, ii, r6r; John, iii, 171 Marg., ii, I56, I6I ; iii, 25 Margery, ii, 161 ; Rich., ii, 161 Sir Will., ii, $152,156-62$, 166 I 72, I $8 \mathrm{r} n ;$ iii, 3 , 19 $n, 25,3 \mathrm{r}$ Will., ii, $157,360,362$; iii, 8 , 22

Harpur, see Harper

Harris, Eliz., ii, 257 ; Fran., ii, 383 Hen., iii, I62; John, ii, 257 Mary, ii, 257: Rebecca, ii, 257 Sarah, ii, 257; Thos., ij, 383 ; iii, 162

Harrison (Harison), Alice, ii, I60 John, ii, 347 ; Rich., ii, 347 Will., ii, 279

Harrold, i, 305, $315 n, 316 n, 382$ 394 ; ii, 92, I I 5, I33, I 44, 147, 154 ; iii, $34,49,54,63,73,114$ adv., i, 388 ; iii, 66,68 ; bridge, iii, $55,63,65,74$; char., iii, 68 ; coins, Rom., ii, 2 ; ch., i, 3I $5 n$, $3 \mathrm{I} 7,324 n, 344,388$; iii, 64,67 hounds, ii, 188 ; ind., ii, 125 iii, 64 ; man., iii, $64-8$; mkt. and
Harrold (cont.)

fairs, ii, 88 ; iii, 35,64 ; mills, iii, 65: pk., ii, I 47; iii, 65: place-names, iii, 64 ; Rom. rem. ii, 8 ; sch., ii, 182 ; iii, 64 ; vicar of, i, 3 I 7 ; woods, ii, 145 ; yeomanry,

Ii, 70
Harrold, Ralf of, i, 365

Harrold Hall, ii, 188 ; iii, 64, 66-7

Harrold Priory, i, $315 n, 349,387-$

90,395 ; ii, $9 \mathrm{I}$; $\mathrm{iii}, 24,38,52$,

$64-8$, Ior, 103, 333 ; prioresses, i, 317 ; iii, $22,74, \mathrm{I}_{3}$

Harrowden, i, 308, 312 ; ii, 153 , 324 ; iii, $33,51,227,234,236$, 237, 279; prehist. rem., i, 33 , 145; iii, 234

Harrowden, Ernest, Ld., iii, 78 ; Thos., Ld., ii, 354 ; Will., Ld., iii, 201

Harston Club, ii, 191

Hart, Lady Jane, ii, 332 ; Rob., iii, 27 I $n$

Harte (Hert), Will., iii, 30, 2r 2

Harter, J. F. H., iii, 276 ; J. H.

iii, 278 ; Steph. J. H., iii, 279 ; Walt. G., iii, 297 ; Walt. G. H., iii, 301

Harteshornc, fam., see Hertshorne

Harthill, ii, 375

Hartley, Will., ii, 29

Hartop, Chas., iii, 212

Hartshorne, man., see Caldecote, Upper

Hartshorne, fam., see Hertshorne

Hartshorne Farm (Ickwell), iii, 247

Hartwell (Hertewell), Abra., iii, 435; John de, iii, I 44, 147 ; Rich., iii, 278

Hartwell Ground (Leighton Buzzard), iii, $4^{\text {I }} 5$

Harvies Manor (Riseley), iii, I58, 159

Harvey, see Hervey

Haryson, see Harrison

Haselden, Alice, iii, 204; Anne, ii, 252 ; iii, 205 : Benj., iii, 205, 208, 209 ; Cath., iii, I45, 205; Constance, iii, $208 n$ i Dorothy, iii, 205; Eliz., i, 357; iii, I46, 205 209; Hugh, iii, 182, 204-6, 216 ; Jas., iii, 209: John, iii, 205: Marg., iii, 205; Mary, iii, 204, 205 ; Rob., ii, 252 ; iii, 204, 205 $208 n, 209$; Thos., iii, 204 ; Will. iii, 204: fam., iii, 204

Haselfoote, Thos., iii, 255 ; Will. iii, 255

Haselhod (Sharnbrook), iii, 9r

Hasclls (Sandy), ii, 197

Hasilden, see Haselden

Hasleden, see Haselden

Haslewood, Edm., iii, 406; Joan, iii, 406-7; John, iii, 406

Haslingfield (Cambs), i, i $79 n, 180$ Hasselden, see Haselden

Hastings, Ada, ii, 211, 238; iii, 300: Ánne de iii, 229: Sir Hen. de, iii, 229 ; Hen. de, ii, 30, 3r, 2 I ; iii, 229, 300, 370 ; Isabella iii, 48; Joan de, iii, 370 ; John de, ii, 238; iii, 229, 233; John Baron, iii, 3oo: Laur. de, see Pembroke, earl of; Sir Ralph, iii 48, 5I, 130, 229: Will. (de), iii, 229 ; fam., ii, 30 ; iii, $4^{8}$

Hasuldene, see Haselden

Haswell, Sewel dc, ii, 212

Hasylden, see Haselden

Hat and bonnet ind., ii, I01, II 8-22

Hatch (Northill), iii, 242, 248, 250

Hatecrist, Will., iii, $5^{8}$

Hather (Lincs), iii, 237

Hatley, Agnes of, ii, 2 r 5 ; Anne, iii, I82; Ellen of, ii, 215 ; Godf., iii, 


\section{A HISTORY OF BEDFORDSHIRE}

Hatley (cont.)

240: John, iii, 182; Rob., iii, I 82, 204, 208; Will., iii, 240

Hatley Port, see Cockayne Hatley

Hatton, Anne, iii, $3^{81}$; Sir Chris., iii, 8, $\operatorname{I0} n, 160,220 n, 28 \mathrm{I} n$, 303; Chris., i, 346; iii, 294; Jane, iii, 86; Jonas, iii, 38I ; Rich., iii, 349 ; Sir Thos., iii, 86

Haughton, see Houghton

Haukes, Cath., iii, 402

Haulsey, Will., ii, 222, 226

Haunce, see Haynes

Haunes, see Haynes

Haveningham, Will., iii, $40_{4}$

Havering, John de, ii, 359; Lucy de, ii, 359 ; Rich. de, ii, 359

Haverings, man., ii, $35^{\circ}, 355 n$, $359-60,364 n, 366$

Haveryngs, see Haverings

Hawburth, Will., i, 370

Haw End (Houghton Conquest), iii, 289

Hawes, John, ii, 40 ; Thos., iii, 25, 3I-3

Hawkins, Anne, iii, 205, 209 ; Geoff., ii, 347 ; Geo., ii, I 70 ; Isaac, iii, 306; Rev. John, ii, 347; John S., iii, 209; Mary, ii, 347 ; Rebecca, iii, I 72 ; Rob., iii, 205. 209; Will., iii, 172, 173,366

Hawksley, Rev. J. W., iii, 95, 142

Hawle, John, iii, 427

Hawnes, see Haynes

Hawnes Grange, see Haynes Grange

Hawte, Marg., Lady, iii, 456 ; Sir Will., iii, 456

Hawtt, Eliz., ii, 319; John, ii, 319

Haye (Hay), Jas., iii, 330; John (de la), ii, 359, 373; iii, 231 Matilda de la, ii, 309, 310, 359, 379 ; Nich. de la, ii, 359; Reg. de la, ii, 359 ; Rog. de la, ii, 359 ; Walt. de la, ii, 309, 31 0, 379

Hayes, man. (Luton), ii, 350, 359

Hayes, Gt. (Podington), iii, 80

Hayes, Little (Podington), iii, 80

Hayes, Will, iii, I 40, 302

Hayes-Millar, R. C., ii, 199

Haynes, i, $318 n, 351 n, 352$; ii $42,44,66, I_{1} 4,306,314,33^{8-44}$; iii, 289; adv., ii, 343; anct. pottery, ii, 8 ; chap., ii, 342 ; char., ii, 344 ; ch., i, $315 n, 378 n$, $391-2$; ii, $289 n, 343$; iii, 289 ; deanery, i, 347; man., ii, 339-4I iii, I2; mill, ii, 34I; Nonconf., ii, 344 ; poorhouse, ii, 104 ; sch., ii, 182 ; vicarage, $i, 316 n$; woods, i, 391

Haynes, Maj., iii, 383

Haynes Grange, man., i, 39I-2 ; ii, $290,34 \mathrm{I}=3$

Haynes Park, ii, 6o, 147, 197, 338, 341,344

Hays, see Hayes

Hayter, Anne, iii, 57 ; Will., iii, 57

Hayward, Tyler \& Co., ii, 127

Haywood (Eversholt), iii, 375

Hazeldean, wood, i, 352

Hazelrigg, Sir Art., ii, 190, I91

Healing, J., ii, r 99

Hearne (Chalgrave), iii, 347

Heath and Reach, ii, II 7 ; iii, 336 399, 400, 402, 4I 7 ; chap., i, 34I : ii, 414 ; char., ii, $182 n$; ch., iii 412 ; man., iii, 405; Nonconf., iii, 4I5; pop., ii, I14; sch., ii, I 82 ; vicarage, iii, 4 I 4

Hecham, see Higham Gobion

Heghham, see Higham Gobion

Heldemar of Tournay, $i, 388$ n

Helder, Rob., iii, 282 n

Hele, John, iii, 189; Rev. Rob.

H. S., iii, I25, 189 ; Thos., iii, 343
Helwell, Marg., iii, 386

Hemiestowe, iii, I20

Hemingford (Hemyngford), John de, iii, 137, 166, 194

Hemp, Alice, iii, 84 ; John, iii, 84

Hemp Land (Maulden), iii, 316

Hemyngford, see Hemingford

Hende, Joan, iii, 231

Hendon (Middlesex), ii, 375

Heneage, Anne, ii, 262; John, ii, 262 ; Geo., iii, 206 ; Geo. F., iii, 206, 209; Thos., ii, 262, 263 , 265,282

Henlow, ii, 97, II 3, 225, 256 $260,261,268,276,280,283$ $285,294,304,324,326$; iii, 69 ; adv., ii, Io6, 285 ; char., ii, 285 ; ch., ii, 283 ; fishery, ii, 283 ; man., ii, $204 n, 283$; mills, ii 283 ; prehist. rems.; i, 174 ; ii 281 ; sch. ii, 182 ; vicarage, i, 316

Henlow End, i, 28I

Henlow Grange, ii, 28r

Henlow Grey, man., ii, 282, 283

Henlow Lanthony, man., ii, $28 \mathrm{r}$, 285

Henlow Warden, ii, 271, 281, 282, 283,285

Henlow Zouches, ii, 225, 282

Henneage, see Heneage

Hennes, Will., iii, I 34

Henry I, i, 349, 37 I ; ii, 23, 88, I 43

iii, $350,352,355,356$

Henry VIII, ii, $38-9$

Henshaw, Hen., iii, 348

Hensman, Hen., iii, 57; Martha, iii, 57

Herbert, iii, I I $4,233,377,423,4^{6}$ Herbert, Ld., iii, 173

Herbert, Auberon, see Lucas and Dingwall, Ld. ; Florence, ii, 328 Geo., ii, $2 \mathrm{I}_{4}$; Rich., iii, I $73 n$. Sir Will., iii, 173, 364 ; Will., ii, 99

Herdewick, see Hardwick

Herdwick, see Hardwick

Herdyng, see Harding

Hereford, earls of, iii, I 55, I66, I 73 : Humph., iii, I 53, I 56, I 68, I 7 I, 172 ; Ralph, iii, 276 ; Will., iii, I 71

Hereford, Rob., bp. of, i, 37I $n$; Thos. Cantilupe, bp. of, ii, 32

Hereford, Cicely, ii, 257, $25^{8}$; Eleanor de, ii, 257; Imbert de, ii, 257, 258; John de, ii, 257 ; Juliana, ii, 257; Margery of, i, 390; Reg. de, ii, 257 ; Rob. de, ii, 257

Hereford's Manor (Wrestlingworth), ii, 256, 257

Hereward the Wake, ii, 22

Herghetone, see Harrowden

Herle, Sir Will. de, ii, 334; iii, 276

Herlingdone, see Harlington

Herlyng, John, iii, 384

Herlyngdon, see Harlington

Herman, see Harman

Hermer, see Harmer

Hermits and anchorets, i, 349

Herne (Herne Grange, Herne Manor), iii, 438, 439, 442, 443

Herne (Heron), Frances, iii, 443 ; Fran., ii, 63, 307, 353 ; Hen., iii, 206, 209; Humph. de, iii, 442

Herne Farm, iii, 438, 439, 447

Heron, see Herne

Herrick, Thos., iii, 361

Herring's Green (Eastcotts), iii, 326

Hert, see Harte

Hertewell, see Hartwell

Hertford, Rich., earl of, iii, I35 : Rog., earl of, i, 394

Hertford, Rich. of, i, 353

Hertshorne (Harteshorne), Eliz., iii, 84,85 ; John, iii, 2 II ; Kath.,
Hertshorne (cont.)

iii, 85 ; Thos., iii, 84 ; fam., iii, 24

Hervey (Harvey, Hervy), Ad., iii 106: Agnes, iii, 158; Barbara, iii, 387 : Charlotte, iii, $3^{87}$; Dorothy Lady, iii, 235, 237 ; Edm., iii, $281,283,302,304,328$; Edw., iii, 387 ; Eliz., i, $35^{8}$; iii, 107 , $283,388,425$; Rev. F. M., ii, I 8 I $n$; iii, I28; Sir Geo., iii, I 5 Geo., ii, 289 ; iii, 97, I07, I 58 , 187; Sir Gerard, iii, 235, 237 ; Gerard, iii, I $5^{8} n, \quad$ I 87,335 Helen, iii, 387 ; Hen., iii, Io6 Isabel, iii, I 54, 281, 283,304 Sir Jarrate, iii, 237; Joan, iii, Io6; John, ii, $54 n, 59,66 n, 69$ iii, 60, I07, I09, 147 , I 55, I 58 I $59, \quad 187,244,245,247,250$ 283, 33I ; Maj. John, ii, 70 John E. A., iii, 244-7 ; Marg., iii 97,283 ; Margery, iii, 283 ; Mary, ii, 289; iii, I06; Sir Nich., iii 273; Nich., iii, I06; Oliver, iii 107, 331 ; Osbert, iii, 106; Sim. iii, 154 ; Steph., iii, 107; Susannah, iii, 250 ; Will., iii, 106 : Will. M., iii, 128; -, iii, 202 fam., ii, 66 ; iii, 104, 237,283 , $33^{8}$

Hervy, see Hervey

Hesding (Hesdin), Ernulf de, i, $287 n$; iii, 347,439 ; Matilda de iii, 439 ; Sibel de, iii, 439

Hesriche, Reg. de, iii, 428

Hesse, Anne, iii, 6r ; Jas., iii, 6

Heton, John, iii, 106, I 73 ; Rich. iii, I73 : Will., iii, 106, 173

Hettenlaia, see Cockayne Hatley

Hevingham, Lady Mary, ii, $342 n$

Hewet (Hewett, Huet, Huett), Sir John, ii, 44; Marg., iii, $28 \mathrm{I}$ Margery, iii, 285; Mary, iii 319: Rob., iii, $285,286,288$ Thos., ii, 257; iii, 281 ; Will. iii, 286,3 I9; - i, $335 n$

Hewyns, Will., ii, 323

Hexton (Herts), ii, 72, 308, 313

Heydon, Ralf, iii, 221

Heydon Hall (Marston Moretaine), iii, 308

Heydon Hill (Marston Moretaine), iii, 308

Heyham, see Higham Gobion

Heyhoe, Thos, iii, 206, 209

Heyne, John, iii, I 79

Heyward, Nich. le, i, 400

Heyworth, see Eyworth

Hibbert, Rob., ii, 357,375

Hibernia, Walt. de, see Ireland, Walt. de

Hibbins, Rev. - iii, 444

Hicchecok, Agnes, iii, 273 ; Will., iii, 273

Hicks, Mrs., iii, 446; - (vicar of Wrestlingworth), $i, 345,346$

Hide, see Hyde

Higgins, Anna M., iii, II 7 ; Chas., iii, I I I, I I 4, I I 7 ; Chas. L. iii, I I 5 I I 7 ; C., ii, 193; F. C. iii, I02; F. G., iii, I04; Hen. L. iii, III, II $n$; Gustavus $F$., iii, 5I, III, I 6 ; John, ii, $60 n$ : iii, IOO, III ; T. C., iii, I II, II6: Lt.-Col. Will. B., iii, I17; W. F. i, I35: Col., ii, I $85 n$; Miss, ii, I85n;-, ii, I94; iii, 12, I I 7

Higham, John de, iii, I $39 n$; Nich de, iii, 408

Higham Bury, ii, 37

Higham Ferrers (Northants), if, 189 ; iii, $40,128,139,143,160$, I $71, \mathbf{I} 82$ 
Higham Ferrers College, iii, 92, 133, 181,182

Higham Gobion, ii, I14, 306, 326; iii, 46 ; adv., i, 360,376 ; ii, 347 ; char., ii, 347 ; ch., i, 3 I $5 n$. ii, $346,371 n$ : earthworks, i, $276,277,278$; ii, 344 ; man., 11 , 330,344 ; mills, ii, 345,346 ; Rom. rems., ii, 8,344 ; vineyard, ii, 345

Higher Berry End (Eversholt), iii,
Hig 375

Higher Rads End (Eversholt), iii,

375
Highfield $208 n$

Highlord, John, iii, 285, 292

Highwood (Luton), ii, 361

Hilier, John le, iii, II 4 ; Margery le, iii, ${ }^{1} I_{4}$; Rob. le, iii, II $_{4}$

Hill (Warden), ii, 43; iii, 254; chap., iii, 255 ; man., iii, 253

Hill (Hille, Hilles), Ad., iii, I 59 ; Alice, ii, 331; iii, 315; Edw., iii, 3r 5 ; Eliz., ii, I02, 330 ; Joan, iii, 167; John, ii, 257; iii, 315 : Martin, iii, 342 ; Mary, iii, 315 ; Maud atte, iii, 428; Rich. (atte), ii, I59; iii, 220, 3I 5, 428 ; Rog., ii, 330; Sarah, ii, 257 ; Thos. (atte), ii, 331; iii, 428 ; Will., ii, 331

Hillersdon, Anne, iii, 28I ; Dennis F., iii, I 89,281 ; Eliz., iii, 187 , 205, 28I, 304, 376; Guy, ii, 364 ; iii, 302 : Harriet, iii, 28I. John iii, 283 ; Marg., iii, 281 ; Mary iii, 283 ; Mich., iii, 302 ; Rich. iii, I87, r89, 205; Sarah, iii, 281; Sir Thos., iii, $376,377,386$; Thos., iii, 187, 279, 281, 283, 376, 384 ; Will., ii, I70; iii, 187,281 , $304,37^{6} ;-$, iii, 20

Hilles, see Hill

Hill Farm (Houghton Conquest), iii, 289

Hill Farm (Old Warden), iii, 253

Hill Foot End (Shillington), ii, 293

Hillgreen Farm (Cranfield), iii 275

Hillingdon, Rog. de, iii, 220

Hillings, the (Eaton Socon), i, 297

Hills, the, earthworks (Meppers hall), ii, 288

Hillsdown, Will., see Hillersdon, Will.

Hills End (Eversholt), iii, 375

Hills Plantation (Tingrith), iii, 435

Hilsden, - ii, 44

Hilton, Ad., iii, 366

Hinchenbrook, Ld., ii, $64 n$

Hind, John, ii, 180

Hinde, Morgan, ii, 337

Hindley, Mary, ii, 304

Hinton, Alice, ii, $363 n$; Geo., ii $363 n$; iii, 325 ; Thos., iii, 325 , 377; Walt., iii, 377

Hinwick, iii, 57, 80-85, I76 ; Rom. rem., iii, 82

Hinwick Hall (Hinwick Manor), iii, $83-5,146$

Hinwick House, ii, 122; iii, 81,82

Hinwick Lodge, iii, 82

Hinxworth (Herts), ii, 214, 223, 225

Hipwell, Dan., iii, 8o; fam., ii, I2

History, political, ii, $x_{7-72}$; social and economic, ii, 73-1 16

Hitchin (Herts), ii, 53, 67, 72, 266, $276,280,286,288,304,334,348$ ch., i, 356; iii, 454; Rom. rem., ii, 5,9

Hithe, John, iii, 25 I

Hiz, riv., i, 28, I 45 ; ii, 26I, 280, 286

Hoare, Sir Hen., ii, 190
Hobard, Sim., ii, $296 n$; Thos., ii, 296

Hobart, Sir Hen., ii, 295, 322 ; Hen., iii, 293

Hobbes (Hobbs), Alice, iii, 355: Eliz., ii, 281 ; Jane, ii, 281 ; John, ii, 28r ; iii, 359 ; Mary, ii. $28 \mathrm{I}$; Rich., iii, 386 ; Rob., i, 367 , $369 n, 370$ : Thos., ii, 35, 89 ; iii, 355,360 ; Will., iii, I IO

Hobbes Edes (Shillington), ii, $295 n$ Hobcote, Will., iii, 245

Hocclive, see Hockliffe

Hoccon, Walt. de, i, 40I

Hocgganclife, man., see Hockliffe

Hocheleia, see Hockliffe

Hockeclyff, see Hockliffe

Hockley (Hockley in the Hole), se Hockliffe

Hockley, John, iii, 392

Hockliffe, i, 305,400 ; ii, 40, 47, 66, II ; iii, $336,344,345,348,349$, $368,383,384,385,421$; adv. i, 4 OI ; iii, 385 ; chants., iii, 386 : char., iii, 386 ; ch., i, 4 or ; iri, 385 Hosp. of St. John, i, 349, 401 402 ; inns, iii, 383,435 ; man., iii 383: Nonconf., iii, 383 ; placenames, iii, 383 ; Rom. Id., ii, 3 sch., ii, 182

Hockliffe Grange, ii, $66 n$; iii, $3^{8} 3$, 385

Hockliffe House, iii, $3^{8} 3$

Hocton, see Houghton

Hoddesdon (Hoddesden), Sir Chris., iii, $403,404,405,408,4^{1} \mathrm{I}, 4^{1} 6$. Ursula, iii, 404, 4II; fam., iii, 414

Hodgkins, John, i, 332

Hodgson (Hogeson), John, ii, 204 Rob., iii, $43^{6}$, $43^{8}$; Thos., i, 370 ; 一, ii, 170

Hodson, John, iii, 56, 68, 8o, ro3, 278

Hogens, John, ii, 325

Hogeson, see Hodgson

Hogg, Maj., ii, r 88

Holand, John de, see Huntingdon, earl of, Sim. de, iii, 2o; Thos. de see Kent, earl of; see also Holland

Holbeche, Hen., bp., see l,incoln, bps. of

Holborn, ii, 159, 163, 165, 172

Holcot, i, 370 ; ii, 96, II 4 ; iii, I75. $176,321,336,386,436$; adv. iii, 388 ; char., iii, 388 ; ch. i, $315 n, 332 n$; iii, 388 ; crown property, iii, 271 ; man., iii, 386; mills, iii, 386,387 ; sch., ii, 184

Holcote, Joan de, iii, I 73 : Rich. de, iii, I73; Steph. de, iii, 153 . Walt. de, ii, 334

Holcot Marston (Bucks), ii, 146

Holcot Mill Pond (Aspley Guise) iii, 338

Holcot Moor, house, iii, 386

Holcot Moors, iii, $3^{86}$

Holcot Wood, iii, $3^{86}$

Holcotwood Farm (Holcot), iii, $3^{86}$

Holcrofte, - iii, 326

Holcutt, see Holcot, Holcote

Holden, Thos., i, $34^{\circ}$

Holderness, earl of, ii, 295

Holecote, see Holcot, Holcote

Holesgrove, Ellen M., ii, 285

Holewell, see Holwell

Holgyll, Will., iij, $15^{8}$

Holland, earl of, ii, 53: Lady, iii 269; Lady Mary, iii, 317 ; Lds., ii, 6r ; iii, $272,29 \mathrm{I}, 3 \mathrm{I} 7$, 318,320 ; Steph., iii, 317

Holland, Sir John, iii, 427 ; Maud, ii, 328 ; Rob. de, ii, 328 ; see also Holand

Holloway, Benj., ii, I70
Holme, i, 308; ii, 201, 209, 2 II, $214,215,235,248,324$; iii, 69 , 260 ; chap., ii, I79; earthworks, ii, 2 Io: mans., ii, 210,234

Holme, Nichola de, iii, 260; Thos. de, iii, 260

Holme Mill Grange, iii, 259

Holme Mills, ii, 209 ; iii, 260

Holme-with-Langford, man., ii, 2 I I, 234

Holt, Anne, Lady, iii, 4II; Sir John, iii, Io7: Rowland, iii, Io7: Sir Thos., iii, 4 I I : Thos., iii, ro7

Holwell (Great Holwell), i, 384 ii, 25, I I 3, 260, 26I, 286, 297 . adv., ii, 287 ; char., ii, 287 ; ch. i, 312 ; ii, 287 ; man., i, 3 I2 ; ii 286

Holwell, Little (Nether), ii, 260 , 293, 294 ; man., ii, 296 ; mill., ii, 297

Holwell (Holewell), John de, ii, 296; Nich. de, ii, 296, 297 ; Pet. de, ii, $276 n$; iii, $231 n$; Rob. de iii, 231 ; Sim. de, ii, 296 ; iii, II2 113 ; Steph. de, ii, 296, 305; Walt. de, ii, 296; 'Will, of, ii, 296

Holwell Bury, i, 307 ; ii, 286, 296

Holwells, man., see South Mills

Holy Sepulchre, order of the, i, 349, $3^{82}$

Holy Trinity, brotherhood of the, iii, 30

Ioly Trinity, hosp. of (Northants), iii, 40,43

Holy Trinity in the Wood, priory of the, see Markyate Priory

Holywell (Clapham), iii, I30

Holywell Priory (Midd.), ii, 221, 222

Home, Hen., i, $26 n$

Home Wood (Eaton Socon), iii, I 89

Honeydon, see Honydon

Honeywood, Matth., iii, 286 ; Rob., iii, 286

Honiton lace ind., ii, 123

Honnor (Honner, Honour), Hen., iii, $407,413,430$; Jas., ii, $281 n$; Jane, iii, 4I3; John, iii, 430 Mary, ii, $281 n$; Thos., iii, 430

Honydon (Honeydon, Camoys), ii, 86; iii, 193, 197; farms, iii, 190,195

Hoo, man., (Pertenhall), iii, 154 , I 55

Hoo, The (Kenıston), iii, 297

Hoo, The (Wootton), iii, 328

Hoo, Thos., Ld., ii, $34 n$

Hoo, Anne de, ii, 355 ; Eleanor de, ii, 355 ; Eliz. de, ii, 355 ; Isabella, ii, 355: Nich. de, ii, $289 n$; Ralph de la, iii, 74: Rob. de, ii $355,360,365$; iii, 3I 4, 3 I 5 ; Sir Thos., i, 400; ii, 36 , 355 ; Thos, de la (atte), ii, 34, 35, 355. 365 ; iir, 3 I 5 ; Walt. de, ii, $289 n$ : Will. de, ii, 355

Hooburne, man., see Hayes

Hoobury, man .(Renhold), iii, 215 , 216

Hoobury (Horbury), Eliz., iii, I4, 45, 215, 330; John de, iii, I4, 215

Hood, Anne, iii, r 35 ; Rich., iii, I 35

Hoo Farm (Wootton), iii, 329

Hoo Hill (Clifton), ii, 276

Hook, John, ii, I73

Hooks, the (Westoning), iii, 455

Hook Wood (Eaton Socon), iii, 189

Hope, Sir John, ii, 67

Hopton, Eleanor, iii, 422 ; Eliz., iii, 405, 422 ; Walt., iii, 405, 422

Hopworth, Dan. R., i, $368 n$

Horbury, see Hoobury 


\section{A HISTORY OF BEDFORDSHIRE}

Hordelhide, ii, 152

Hore, Thos., i, 398

Horepol, Rob. de, ii, 22

Horim, Alwin, ii, 328

Horle, Eva de, ii, 81 ; Rob., ì, 8 r

Horley, Rev. Edw., iii, zor

Horne, Anne, iii, 61; Thos., i, 398; Will., iii, 61 ; 一, ii, $63 n$. 64

Horne Lane (Bed.), Angl.-Sax. rem., i, 186; Rom.-Brit. rem., ii, 5

Horsehill Farm (Harlington), iii, 379 Horsetails (Equisetaceae), i, 56 Horsley, iii, 63

Horthornes (Woburn), iii, 461

Horwodys (Cople), iii, 240

Hospitallers (Knights of St. John) i, $314 n, 315 n, 349,373,394$ $306-8400,401,402,403$; i1, $144,204,235,277,278,288,290$ $305,337,362,377,379$; iii, 23 $24,31,64,75,85,90,95,107$ $11_{4}, 135,142,143,147,154$ $157,158,160,165,167,176$ $184,19011,198,200,201,212$ $240,245,246,258,260,264,286$, $302,315,318,324,332,350,376$, $37^{8}, 383,3^{8} 4,385,396,420,437$, $444,44^{6}$

Hospitals, i, 396-403 ; iii, 6, 149

Hotofts, man. (Cople), iii, 240

Hotot, lsabel (de), ii, 321 ; iii, 27 r, 290 ; Joan, iii, 112 ; Juliana, iii, I12 ; Rob., iii, 98, I1 ; Thos., iii, 112 ; Will (de), ii, 321 ; iii, I12, $113 n, 271$

Houghton, Ad., iii, 385 ; Hugh of, iii, 362: Isabel de, ii, 32 I, 324 ; iii, 27I, 317 ; John, i, 319, 320, 348 ; ii, 29: Rebecca, iii, 385 ; Kob., i, 384 ; Will. de, ii, 321 ; iii, $271,3^{17}$

Houghton, brook, iii, 329

Houghton Conquest, ii, 66, 115 ; iii, $14,267,270 \mathrm{n}, 288-96,373$, 389 , 393 ; adv., iii, 295 ; char., ii, 180 ; iii, 296 ; ch., iii, 273,294 381 ; crown property, iii, $27^{1}$; hayward of, ii, 86 ; man., ii, 147 ; iii, 290 ; man.-louse, iii, 289 ; mills, iii, 289 ; Nonconf., iii, 296; pk., ii, 147 ; iii, 290 ; place-names, iii, 290 ; poorhouse, ii, $\mathrm{rO}_{4}$; sch., ii, 180,182

Houghton Franchise, iii, 295, 296

Houghton Gildable, iii, 295, 296

Houghton Grange, man., iii, 29x, 293

Houghton Hall, iii, 367, 368, 389

Houghton House ii, 6r, 64; iii, 289,290

Houghton Lodge, ii, 4 I

Houghton Regis, i, I74; ii, I02, $\mathrm{rr}_{4}, \mathrm{r}_{5} \mathrm{r}, 314$; iii, $336,337,350$, $351,352,355,368,372,389-93$; adv., i, 314; iii, 393; chant., i, 329 ; ii, 150, 151 ; iii, $391,394:$ char., iii, 394; ch., i, 311, 314 $315 n, 329 n, 332$; ii, 150, I5I iii, 392 ; gild, i, 330 ; grange, ifi 394 ; hayward of, ii, 86 ; hound money, ii, I $43 n$; ind., iii, 390 man., ii, $23,150,178$; iii, 370,371 390 ; mills, iii, 389 ; Nonconf., iii, 389 ; place-names, iii, 390 ; prehist. rem., i, I46; Rom. rems., ii, 8 ; schs., ii, 150, 151, 156, 182; iii, 394 ; springs, $i$, I9 : vicarage, $i$, $316 n, 341$

Houghton Regis, ldship of, i, $37 x$ $37^{2}, 37^{6}$

Houghton Regis, Upper, iii, 352, 389

Houlden, Rev. 一, iii, $29 n$
Hour-glass, iii, 47, 266

Houston, R., ii, 265

Houstone, see Houghton Conquest and Houghton Regis

Houton, see Houghton Regis

Hoveden, Rob., iii, 424

Hovell, Le, close (Wootton), iii, 33 I $n$

Howard, Lady Lucy, iii, 441 ; Lady Mary, ii, 347, 38z ; Edw., Ld., ii, $347,3^{82}$

Howard, Chas., ii, 138 ; Sir Fred. ii, $66 n$ i iii, 6 ; Geoff., ii, $66 n$ : Harrold, ii, $66 n$; Jas., ii, $66 n$, 126 ; iii, 9, 128, 130; Jas. H. iii, 297: John, ii, $65,66 n, 103$ $108,125,126$; iii, $5,7,8,20$ $30,87,233,234,238,285,383$; see also Norfolk, duke of; John C., iii, $23^{8}$; Lucy, ii, 277 ; Marg. ii, 340 ; Phil., iji, I92: Sir Rob. ii, $34^{\circ}$; Thos., see Surrey, earl of, and Norfolk, dk. of ; Will., see Stafford, visct.

Howard Villa (Cardington), iii, 233

Howbury Hall (Renhold), ii, 65, $66 n$; iii, $214,215,216$

Howe, vsct., iii, 444

Howe, Will., iii, 313 ; 一, ii, 15

Howe and Pertenhall, man. (Pertenhall), iii, I 54,155

Howell, Jos., ii, 317, 319

How End, man. (Houghton Conquest), iii, 293

Howgill, Rob., iii, 266

Howland, John, ii, 359

Howlett, - ii, 25 n

How Wood (Stagsden), iii, 96

Hubald, Hugh, iii, 138 , 159

Huckell, Agnes, ii, $285 n$

Huddleston, Sir John, ii, 382

Will, ii, 382

Hudibras' Hole (Cople), iii, 238

Hudnall, man. (Studham), iii, 427

Hudson, John, ii, 204 ; 'Will., ii, 204

Huet, Huett, see Hewet

Hugate, Will de, i, 39r, 393

Hugeford, see Huggeford

Hugesson, Sir Will., iii, 219

Huggeford (Hugeford), Alice, iii, 224 ; Margery, iii, 5O, 2II, 224, 330 ; Sir Will., iii, 50, $33^{\circ}$; Will., iii, 50, 211, 224

Huggyns, Jane, ii, $359 n$; Will., ii, $359 n$

$\mathrm{H}$ ngh, St., see Lincoln, Hugh of Aralon, bp. of

Hugh, ii, $282,283,326,383 n$; iii, $46,113,223$; archd. of Beds, $i$, 313; monk, i, $364 n$

Hughes, Isaac, iii, 372

Hulcote, see Holcot

Hulier, Pet., iii, 146

Hull, Rev. Rich., ii, 305 ; Will., iii, 156

Hulls (Pegsdon), ii, $295 n$

Huls, Will., ii, 305

Hulston, John, ii, 245

Humberdale, iii, $60,144,146$

Humbershoe, ii, Ir $4,314,3$ I 8 ; iii, 362 ; chap., i, $318 n, 326 n$ : man., ii, 318

Humbrichesho, see Humbershoe

Hume, Sir Patrick, ii, 217,218

Humphrey, iii, go

Humphrey, Anne, ii, 385; John, iii, 427 ; Matilda, iii, 427

Humphreys, Rev. -, ii, I68; ii, 257

Hunderigg (Bucks), chap., i, 318

Hunderigg, Thurstan of, $i, 371 ;$

Hundon, Thos., ii, 236

Hunesdone, see Hunsdon
Hungate, Thos., iii, 454

Hunger, John, iii, 95

Hungerhill (Marston Moretaine), iii, 308

Hungerhill Spinney (Podington), iii, 80

Hunsdon (Hunesdone), ii, I5

Hunsdon, Lds., Geo., iii, $3^{1} 4$; Hen. iii, $3 I_{4}$; John, iii, $3 I_{4}$

Hunstanton Convalescent Home, ii, 246

Hunt (Hunte), Anne, iii, 220; Eliz., iii, 220 n: Jane, iii, $4 \mathrm{r} 6$; Mary, iii, 220 ; Rog. (or Rob.), iii, 220 ; Thos., ii, $327 n$; iii, 220 ; Will., iii, $220,44^{8}$

Hunter, Geo., i, 334

Huntercombe, Ellen, ii, 255 ; Walt., ii, 255 ; Will. de, ii, 255

Huntingdon, $i, 294 n$

Huntingdon, hon., i, 387 ; ii, 30 , $210,226,238,246,247 n, 255$; iii, $23,42,46,59,61,64,126$, $134,150,166,195,234 n, 240$, $260,280,290,297,314,326$

Huntingdon, archds., i, $324,334 n$, 397 ; priors, ii, 219 ; iii, 134,168 , 169

Huntingdon, ctsses, of, ii, 36 ; Constance, i, $388 n$; Judith, wife of Earl Waltheof, see Judith, ctss. ; Maud, ii, 237; earls of, iii, . $6 n, 74$; David, iii, 195 . Hen., i, 388; iii, ro2; John, ii, $210,237,238$; iii, 101, 102 Sim., ii, 255: Tosti, it, 237 Waltheof, ii, 21 $n, 22,155,237$; iii, $280,338,408$

Huntingdon, Christine of, i, 358 , 359 ; Hen. of, i, 313; ii, $24 n$; Rob. de, iii, 194

Huntingdonshire, lace ind., ii, 123 land measurement, ii, 77; wool ind., ii, 91

Huntingfold, Godf. de, iii, 247 ; Hen. de, iii, 247; Joan de, jii 247; Sibil de, iii, I $76 n$; Walt. de, iii, 247

Hurdlow (Derb.), i, r8 8 n

Hurley, John, Ld., iii, 44I

Hurley, Thos., iii, 293

Hurst, Will. de, i, $386 n$

Husborne Crawley, ii, II 4, 324 iii, $308,320,394-99,45^{8}$; adv. i, 376 ; iii, 398 ; char., iii, 398 . ch., i, $315 n, 332 n, 371 n$; iii, 397; crown property, iii, $27 \mathrm{I}$ man., i, 377 ; iii, 395 ; nills, iii, 394 ; sch., ii, $r 83$; treasure trove, iii, 395 ; vicarage, i, $316 n$

Husc, Geoff. de la, iii, 57 ; Godf. de la, iii, $5^{8}$; Thos. de la, iii 57,85

Huskey, J. R. T., iii, $x_{4} 6$; Rich. iii, 146

Husseburne Crawel, see Husborne Crawley

Husy, Sir John. iii, $272 n$

Hutchinson, Eliz., iii, 25I ; John iii, 12 ; - ii, 207

Hut-circles, i, 270

Hutton, Will, , iii, 276

Huxley, Eliz., iii, 376 ; Jas., iii, 28r : Sir John, iii, 37r: John, iii, $371,372,376,407$; Sarah, iii, 408 ; Thos., iii, 372 ; Will., ii, 158

Hyde, East and West, ii, 306, 308, $348,349,350,359,360,374$; chs., $\mathrm{i}, 346$; ii, 374 i mans., ii, $350,357,363$; mill, ii, 366

Hyde, La, man. (Upper Gravenhurst), ii, 33 t

Hyde, The, man. (Studham), iii, $427-9$ 


\section{INDEX}

Hyde (Hide), Agnes de la, i1, 357 Alan de, ii, 357; Aylward de la, ii, $357 n$; Fulk de la, ii, 357 ; Hawisia de, iii, 429; Hen. de la, ii, 357 ; John, ii, 3 ro ; iii, 374 n; Maude, iii, 287 ; Petronilla, ii, 357 n; Rog. de la, ii, 357: Thos. de la, ii, 357, 359; Walt. de, ii, 357 ; Will., iii, 118,429

Hyde Aynel, West, man., ii, 350, 360

Hyde mill (Luton), ii, $357 n, 366$

Hydewood (Studham), iii, 429

Hylston (Ilvestone), Rich., i, 398

Hyndeman (Hyneman), Rich., i, 398, 399; ii, 332

Hynton, see Hinton

Ibbot, 一, iii, $\mathrm{IO}_{4}$

Iberians, i, 1 58-9

Iceni, the, iii, $176 n$

Ickleford (Herts), ii, 287, 304

Icknield Way, i, I 59, I61, I 73, I76, 269,280 ; ii, $3,7,17,18,23,314$; iii, $350,351,356,390,44^{8}$

Ickwell, i, 394; iii, 245, 246; fair, iii, 246 ; man., i, 394 ; iii, 245

Ickwell Bury, ii, $66 n$; iii, 242, 244, 246

Ickwell Green, iii, 245

Ickwell House (Northill), iii, 242,

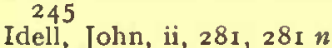

Ilefeulde, Will. de, iii, I 73

Illyngworth, Geo., iii, 6 ; Rich., iii, 6

llvestone, see Hylston

Impey (Impie, Ympie), Sam., iii, 385 ; Thos., ii, 40 ; iii, $346,34^{8}, 3^{8} 5$ Implements, bronze age, i, I69; Celtic, i, 170 ; ii, 4 ; neolithic, i, 30, 3I, I6I-167; ii, 308 ; iii, 350 ; palzolithic, i, 29, 30, I 45-6, I5I-I 59, I7I; ii, 25I, 3I 4, 348 ; iii, $36,234,297,350$; post-glacial, i, 28 ; Rom., i, 172 ; ii, 7, 12-15 Incent, John, ii, 157

Inclosures, ii, 96

Industries, ii, 11 7-27

Inge, Edw., ii, 315; Fremund, iii, 452 ; Isolda, iii, 452, 453 ; Joan, ii, 336 ; iii, 449 , 452 ; Marg., iii, 452 ; Rich., ii, 316 ; Thos., iii, $252,430,449$; Sir Will., ii, 285 ; Will., i, $325 n$; ii, 336 ; iii, 437 , $449,452,453,454$

Ingewar, ii, 226

Ingleton, Geo., iii, 91 ; Joan, iii, 9I ; Rob., iii, 9 I

Inglis, Cath., iii, 4I 8 ; Mary, iii, 421 ; Sir Hugh, iii, 418; Hugh, iii, 41 7; Mary L., iii, 42I ; Sir Rob., ii, 66: Sir Rob. H., iii, $42 \mathrm{I}, 423$; Rob. H., iii, 4I9; -, Lady, iii, 419

Ingram, Rob., iii, I54

Inlonde (Southill), see Ireland

Inns, John, ii, 224

Inscriptions, ii, $218,233,254 n$, $266,273 n, 275,287,299,324$ $33 \mathrm{r}, 332,338,343,359,372,373$; iii, $25,27,28,39,47,53,75,76$, $86,103,120,127,135,138,143$, $149,179,185,188,193,235 n$ $24 \mathrm{I}, 250,266,273,289,295,307$ $319,328,340,351,366,373,388$, $411,425,434,438,450$; Roman ii, 6,7

Insectivora, i, I 39

Insects, i, $7 \mathrm{I}-88$

Inskip, Thos., ii, I 1, I3, I4

Inwards, R. W., iii, 306

Inwen, Sarah, iii, 332 ; Thos., iii 332
Inwode, close (Ravensden man.), iii, 210

Inwood (Haynes), ii, 34I, 342

Ion, see Gravenhurst, Lower

Ion House (Lower Gravenhurst), ii, 337

Irchester (Northants), i, I 8

Ireland (Southill), iii, 256

Ireland, Walt., i, $325 n$; iii, 132,136

Iremonger, Frances, iii, 406 . Humph., iii, 406 ; John, iii, 406 . Judith, iii, 406 ; Marg., iii, 406 : Martha, iii, 406; Thos., iii, 368 , 406 ; Will., iii, 406, 409

Ireton, Col., ii, 5I, 52 ; iii, 4

Iron Age, $i, 170,182$; interments, i, 182 ; vases, i, I 70,174

Isaac, Edw., iii, 207

Isabella, dau. of Edw. III, ii, 39; queen, ii, 33 ; iii, I6, 353

Isabella ' inclusa', $\mathrm{i}, 350$

Isham, John, iii, 4 I 6

Islip (Islep), Sim. de, iii, I25 ; Will., iii, 91,92

Ispannia, Rob. de, ii, 267

Issoudun, Alice, ii, $233 n$; Maud ii, $223 n$; Ralph de, ii, $223 n$

Ivel, canal, ii, 276

Ivel, riv., i, 28 ; ii, 7

Ivell, Thos., iii, 248

Ivinghoe (Bucks), iii, 368

Ivo, iii, I 44

Ivory, John, ii, 286 ; Rob., ii, 286 Will., ii, 286

Ivota, $\mathrm{i}, 354 n ; \mathrm{iii}, 28$

Ivri, Acard de, iii, 338 ; Ralph de, iii, 339 ; Reg. de, iii, 339

Jackman, Clem., iii, 406: Eliz., iii, 407; Gabriel, iii, 407; John, iii, 406: Reg., iii, 407; Rob., iii, I55; Will., iii, $400,407,4$ II

Jackman's Farm (Goldington), iii, 202

Jack's Lane (Turvey), iii, I I $4 n$

Jackson, John, ii, 262 ; Rob., ii, 204 ; Lt.-Col. S., ii, 70; Rev. Will. H., iii, Ioo

Jacob, iii, 363

Jacobs, R., ii, 198

Jakes, Thos., $\mathrm{iii}, 44^{8}$

James I, king, ii, 40; iii, 42, $\mathrm{IO}_{7}$ 289,290

James, Giles, ii, I63, 164: 一, ii, 120

Janaway, Thos., ii, 24I

Janyns, see Jennings

Jarman, Eliz., iii, 428; Will, iii, 429; see also Jermayn

Jarpenville, Will., iii, 137

Jeeves, Messrs., ii, II 8

Jefferies (Jeffries), John, ii, 255; iii, 109 ; Rob., iii, 109: Thos., iii, I09

Jempsis, man., see Gemys

Jenkin (Jenkyn), John, iii, 335; Rob., ii, 3 Io

Jenkins, 一, ii, I94; iii, 202

Jenkinson, Ant., iii, 332

Jenkyn, see Jenkin

Jenney, Edm., ii, 296

Jennings (Janyns), John, iii, 442 ; John W., iii, $3^{81}$; Sir Steph., ii, I59; 一, ii, I 33

Jermayn, Thos., iii, I54; see also Jarman

Jermye (Jermy), Eliz., ii, 323; iii, 244 ; Fran., ii, 323 ; iii, 244

Jervis, Eliz., iii, 384 ; Will., iii, 384

Jessop (Jessopp), Thos., i, 342

Jeune (Jeuene, Juvene, Juveni, Juvenis), Agnes le, ii, 318; Barth. le, ii, 32, 3I8; Giles le, ii, 318 ; Isabella, ii, 318 ; John
Jeune (cont.)

de, ii, 232; iii, 358, 457 ; Rich. ii, 318 ; iii, 362 ; Thos. de, ii, 232

Jewelsbury Manor, see Graven. hurst, Upper

Jews, i, 321 ; iii, 338,363

Jobson, Sara, ii, 358 ; Stafford, ii, $35^{8}$

Joce, John, iii, 248 ; Rob., iii, 248 ; Walt., iii, 286 ; Will., iii, 248 see also Joyce

John, king, iii, 352

John, parson of Haynes, ii, $289 n$; the smith, iii, 383

John of Gaunt's Hill (Sutton Park), i, 300

Johns, Phil., ii, $4^{\circ}$

Johnson, Anne, ii, 277 ; iii, 441 Cath., iii, 418; Fran., ii, 99 Geo., iii, 325; Sir Hen., ii, 277 iii, 44I ; Hen., iii, 418; Dr. Jas. iii, 87, 328; Jerome, ii, 335 ; John, ii, I56; Jos., iii, 418 ; Martin, ii, I I 8, $349 n$; Mary, ii, 378 ; Rich., iii, 442, 454 ; Rob., iii, 424; Sam,, iii, 257 ; Thos., ii 378 ; iii, 307, 418, 448; Will., ii, 39 ; iii, 408, 418 ; 一, iii, I25, 323

Jolliffe (Joliffe), Ann, iii, 66, 68

Jones, A. O., ii, I 99, 200 ; Chas, iii, 307 ; Griffith, iii, 202 ; Rich., iii, 307, 4I5; Rowland, ii, I 99

Jordan, iii, I 55

Joyce, Rob., ii, 316; R., ii, 199 Thos., ii, 43, 344: see also Joce

Joye (Joy), Will., ii, I55; iii, 3o, 2 I $5 n$

Judd, Sir Andr., ii, I 57

Judith, ctss., i, 31 3, 353 ; ii, I 53, 2 I 0 2 I I , 2 I G, 226, 237, 239, 246, 247 277 ; iii, 42, 46, 47, 59, 61, 64, 92, $96 n, 126,150,195,229,234 n$ $236,240,260,280,297,302$

Julyan, Thos., ii, 377 ; iii, I26 ; Rich., iii, I 26

Jurden (Jurdon), Hen., iii, 226 ; Wili., iii, I2 I, 325

Juvene (Juveni, Juvenis), see Jeune

Kadington, see Caddington

Kancia, Jas. de, iii, 246 ; Thos. (de) (of), iii, 246, 248 ; see also Cantia Kane (Cane), Barbara, ii, 289 ; Rich. de, ii, 32 I

Kantia, see Cantia

Karleton, see Carlton

Karun, fam., see Carun

Kateden, see Caddington

Katherine, see Catherine

Kaye, Cecil W., ii, I 77

Keale, Sybil, iii, I 44

Keaynsham, see Keynsham

Keblewhite, John, iii, $376 n$

Kebyle, Will., ii, 226

Keeble, Geo., iii, 264, 266; Jas., iii, 264 ; Messrs., iii, 240

Keeley (Wootton), iii, 328, 329, 33

Keeling (Kelyng), Antelminelly, ii 270; Sir John, iii, 258, 26 I John, ii, 270 ; iii, 261 ; Serjeant, ii, 55,56

Keene, Jas., iii, 76, 24I, 242, 4I 4 420 ; John, ii, 229 ; iii, 283,288 ; -, iii, 334

Kelway, Rob., ii, I5 I

Kelyng(e), see Keeling

Kemble, J. M., i, 184

Kemhestun, see Kempston

Iemmeston, see Kempston

Kempston, i, 354; ii, $21,30,44,70$, 96, I 15 ; iii, 2 I $n, 23 n, 25$ I, 267 , $284,290,296,302,308,326,333$, 


\section{A HISTORY OF BEDFORDSHIRE}

Kempston (cont.)

335; adv., iii, 304 ; A.S. rems., i, $176,177,178,179,180,181,183$ $184,185,188,189$; iii, 297 ; char., iii, 305 ; ch., i, $315 n, 357$; iii, 297, 303, 304; man., ii, 2 I $n$ iii, 297; manuf., ii, 127; mill, iii, 297; Nonconf., iij, 297 ; palæolithic implements, $i, 145,162$, 165, 174; iii, 297 ; Rom. rems., ii, 8 ; iii, 297 ; sch., ii, I83; iii, 297,305 ; vicarage, i, $316 n$

Kempston, Geo., iii, 216 ; Will. (of), iii, 23, 216

Kempston Borne, iii, 304

Kempston-Daubeny, man., iii, 297, $298,299,304$

Kempston Greys, man., see Kempston Hastingsbury

Kempston Hardwick, iii, 297, 299, 300,301

Kempston Hastingsbury, iii, 300, 301 ; mill, iii, 303

Kempston Wood, iii, 329

Kempton, ii, I 39

Kendale, Beatrix de, ii, $256 n, 354$ Edw. de, ii, 256, 354, 357; Eliz. de, ii, 256 ; Isabella de, ii, $256 n$; Jordan de, ii, 356 ; Marg., ii, 255 , 256 ; Rob. de, ii, 255, 328, 354, 356 ; Thos., ii, 354

Kendall, Anne, iii, 3or ; Rob., iii, 301

Kendals man. (Wrestlingworth), ii, $255,256,257$; mill, ii, $256 n$

Kenninghall (Norf.), $i, 183,189$

Kensington, iii, 368

Kensworth, ii, 3, 71, 314; iii, 367, $368,390 n, 426$; man., ii, 23 : prehist. rem., i, I66; Rom.-Brit. rem., ii, 1,8 ; sch., ii, 183

Kensworth Lynch, ii, 3

Kent, ctsses. of, ii, 327 ; Amabel, ii, $322,324,345$; Arabella, ii, 332 ; Eliz., ii, 26I; Joan, iii, I0I; Marg. iii, 339; Mary, ii, 332 Susan, iii, 232 ; Jemima, dchss. of, iii, 66 ; dks. of, ii, 62,337 , $376 n, 377,378,379,380$; iii, 66,68 ; Hen. Grey, ii, 327,332 ; earls of, i, $335 n$; ii, 44, 324 ; iii I $6 n, 65,290,321,323,326$; Ant., ii, 56, 290, 325, 327 ; Chas. ii, $327,330,337$; iii, 228,232 . Edm., ii, 37, 283, 327, 331 ; iii, 3. IOI, 272; Geo. ii, 37, 267, $296 n, 327,332$; Hen., ii, 61, 261 $283,305,327,329,330,332,334$, 337 ; iii, $65,105,229,396$; Hub. de Burgh, ii, 283 ; Reg., ii, 327 ; Rich., ii, 37, 266, 322, 323, 327 ; iii, 396, 436; Thos. de Holand, iii, IOI

Kent, Edw., ii, 285 ; iii, 243 ; Eliz., iii, 243 ; Thos., ii, $285,378,379$, 380 ; tam., iii, I9 4

Kentish, Thos., ii, 270

Kentwode, Reg., iii, 230

Kerdinton, see Cardington

Kerdyngton, see Cardington

Kersey, Joan de, iii, 292 ; Rich. de, iii, 292

Kershaw, J. C., ii, 198

Keston, Thos. de, ii, 355

Ketenho, ii, $357 n$

Kettell, John, iii, 340

Keynes, Alice, 1i, 346,379 ; Christina, ii, 346, 379: Eleanor de, iii, 272: Emma, ii, 346, 379 Joan de, ii, 346 ; Margery de, ii 346,379 ; Will. de, ii, $346,378 n$ 379

Keynsham, Anne, ii, 252; Eliz. ii, 252 ; Geo., ii, 40, 252, 2 . 3

Keyrun, see Carun
Keysoe, i, 297, 308; ii, 115,246 ; iii, 123, 128, 1 36-1 39, I 53 : adv. iii, I39; char., iii, 139; ch., i, $315 n, 392$; iii, 138 ; earthworks, i, 305,306 ; iii, 136; man., iii, 14 , 136; mill, iii, 136; rectory, i, 393 ; sch., ii, I83 ; vicarage, i, $316 n$

Keysoe Bury (Berrysted), man., iii, $136,137,13^{8}$

Keysoe Grange, man., iii, I37

Keysoe Park Farm, iii, 136

Keysoe Row, iii, I36

Killbere, Sim., iii, 3

Kim, riv., ii, 71 ; iii, 153

Kimbolton (Hunts), ii, 39, 71, 200 : iii, 40,124, 136, $157,160,171$ 202, 209: Catherine of Aragon at, ii, 38 ; man., iii, 168, I 7

Kimbolton, hon., iii, I 53, I55, 166, I 7 I , 172, I 73, 296

Kimpton, Edm., ii, 204; Eliz., ii, 204; Geo., ii, 203, 204; Lucy, ii, 204: Will., ii, $342 n$; fam., iii, 406

Kimptons, man. (Stanbridge), iii, 406, 407

King (Kinge, Kynges), Eliz., iii, I 57 ; Eulalia, iii, $157 n$; John, i, 402 ii, 385 ; iii, 157 ; John E., ii, 176 ; Smith, iii, 255; Thos., i, 398 . ii, 379 ; iii, 278 ; Will., ii, 381

King John's Well (Odell), iii, 74

Kingsbury (Dunstable), i, 360; ii, 23 ; iii, $35 \mathrm{I}, 352,356$

Kingsbury, Thos., i, $353 n$

Kingsbury House (Dunstable), iii $351,368,416$

King's Coll. (Camb.), i, 400 ; ii, 357 $35^{8}$

King's Ditch (Bedford), i, 281; iii I, 6, 8, 9

King's Hall (Camb.), i, $326 n$; iii, 61,63

Kings Langley (Herts), priory, i, $376 n$

Kingsley, Eliz., iii, 166; Hen., iii. I66; Heylock, ii, 244; iii, I66

Kingsmead (Bedford), iii, 8

King's Way (Ridgmont), iii, 32r

Kingswood (Chamois Park) (Eaton Socon), man., iii, 195

King's Woods (Leighton Buzzard) iii, 400,405

Kinnardseye, John de, ij, 247

Kippest, Rob., iii, 201

Kiriel, Bertram de, iii, 452 ; Eleanor de, iii, 452

Kirke (Kyrke), Alex., iii, 437 Rich., ii, 301; Will., iii, 437

Kirkeby (Kirkby, Kyrkeby), Christine de, iii, 40, 194, 219; John, i, 402 ; see Ely, bps. of; Mabel, iii, 219, 220 ; Marg. de, iii, 194 ; Rob. de, ii, 337 ; Will. de, i, $326 n$, 353; iii, 40, 194, 2I9, 220

Kirton, Alan de, iii, 37 ; Grace de iii, 37 ; fam., iii, 39

Kirtons (Biddenham), see Ford End

Kitchen, man. (Pulloxhill), ii, 378

Kitchen End Farm (Pulloxhill), ii, $37^{6}, 37^{8}$

Kitson (Kytson), Eliz., ii, 277 Hen., ii, 277; Marg., ii, 277 ; iii, 318 ; Sir Thos., ii, 277 ; 一, iii, 355

Knapp, Constance, iij, $208 n$; John, iii, 208

Knight, Dan., ii, 373 ; Hen., ii, 364 ; John, ii, 343 ; iii, 376,377 ; Jos., iii, 431 ; Rich., iii, 376, 377 ; Sim., iji, 363

Knightley, Anne, ii, 234 ; Sir John, iii, 367 ; Sir Valentine, ii, 234
Knighton, Anne, ii, 317 ; Sir Geo., ii, 317 ; Thos., iii, 4 II

Knights Templars, see Tempars

Knoll, the (Aspley Guise), iii, 338 ;

Knotting, ii, 96, I1 5 ; iii, 123, I39I 42 ; adv., iii, I 42 ; chap., i, 318 ; iii, I42 ; char., iii, I42 ; ch., i, $315 n$; iii, 139; inclosure, ii, 97 ; man., iii, 95, 139-142, 401 ; sch., ii, 183

Knotting Green, iii, I 39

Knottyngges, see Knotting

Knowles, Thos., ii, 99

Koc, see Cocke

Kym, riv., see Kim

Kyme, Maud (Matilda), de, ii, 35I, $352,352 n, 355,365$; Will, de, ii, $35 \mathrm{I}$

Kympton, see Kimpton

Kynaston, Edw., ii, 262

Kynbell, Nich., ii, 296 r

Kynges, see King

Kyrke, see Kirke

Kyrkeby, see Kirkeby

Kyrkham, Amicia de, ii, 287 : Amy (Anne), iii, 429: John (de), ii, 287 ; iii, 429

Kytson, see Kitson

Lace-making, ii, $38 n, 108,122-124$, 213 ; iii, $5,35,64,69,82,89,94$, $96,100,117,124,128,149,157$, $234,280,297,305,309,435,45^{8}$

Lachebury, Will., i, 400

Lacies, man. (Clifton), ii, 277

Lacu, John de, i, 384

Lacy, Agatha (Alice) de, iii, I82 : Hen. de, ii, 277: John de, ii, $277,37^{8}$; Sir Rich. de, iii, I 82 ; Roland, iii, II9

Lady Mary's Well, see Ladywell

Ladysgrove (Soulsbury), iii, 4I 5

Ladywell (Turvey), iii, I I $4 \boldsymbol{n}$

La Hyde, man. (Upper Gravenhurst), see Hyde

Lake, Sir Thos., iii, 2oI : Thos., ii, 256: Will., iii, I67

Lalega, Lalegh, La Leye, see Thurleigh

Lalleford, man. (Luton), ii, 35o, 363

Lamar, John, ii, 373

Lamb (Lambe), Barbara, iii, 44ㅇ, 445 ; Hen., iii, 45I ; Sir John, iii, 440 ; John, iii, 45 I

Lambert, Jane, ii, 318 ; John, ii, $318 ; \ldots$, ii, 318

Lambert's Spinney (Stagsden), iii, 96

Lamb's Farm (Renhold), iii, 218

Lameleye, Rog. de, iii, 244

Lampslandes, le (Renhold), iii, $217 n$

Lancaster, duchy of, ii, 238,250 256, 257 ; iii, 371

Lancaster, earldom of, ii, 2 I $n$

Lancaster, John of Gaunt, dk. of ii, 248; iii, 102; Hen., earl of, ii, 33 ; iii, 3 , Ior ; Thos., earl, ii, $247,300,339,384$; iii, 2 , I 2

Landas, Juliana de, iii, 456 ; Nich. de, iii, 449; Valentina de, iii, 449 ; Will. de, iii, 449

Landimareswell, see Ladywell

Landon, Rog., iii, 54, 315, 425

Landpark Wood (Totternhoe), iii $44^{8}$

Lane, Mich. in le, iii, 95: Sir Ralph, iii, 98 ; Ralph, ii, 295 ; Ricb., ii, 281 ; Rob., ii, 295 iii, 98; Will., iii, 17 ; Mrs., iij, 228

Langbain, - , ii, 170

Langdate, John, iii, 209

Langeho (Gt. Barford), iii, $182 n$ 


\section{INDEX}

Langeley, see Langley

Langenhoe, Sim. de, iii, I 47

Langetot, Ralph (de), ii, 220, 22 I, 267,275

Langford, ii, 75, II3, I38, 20I, 209,2 II, 234, $282 n, 326 n$; iii 69 : adv., ii, 237 ; Brit. coins, i, 173: ii, 234: char, ii, 237 ch., i, 3I5 $n, 324 n, 349,394 n$ ii, 234,236 ; iii, 70 ; man., i, 394 ; ii, $143,204,210 n, 211,216,234$; iii, 258; mills, ii, 235; poorhouse, ii, I04; prehist. impls. i, 145; ii, 234 ; sch., ii, 183 vicarage, $i, 3 I 6 n$

Langford, Christian, iii, 425 ; John, iii, 425; Ralf de, i, 358; Rob. iii, 425 ; fam., iii, 424

Langford End (Tempsford), ii, 25

Langford Rectory, man. (Langford), ii, 234, 235, 237 ; mill, ii., 235

Langhill (Gt. Barford), iii, I $82 n$

Langhorne, Dan., ii, I65; Eliz., iii, 22 :Thos., iii, IO2; Will., iii, 22

Langley, Edw., iii, 329 ; John, i 330; Rich., iii, 277; Sir Rog. ii, 345: Sir Will., ii., 345

Langleys, man. (Luton), ii, 350, 363

Langston, Thos., iii, 377

Langton, John de, iii, 91 ; Thos. de iii, 91; Walt. (de), bp., see Coventry and Lichfield, bps. of

Langtons, man. (Sharnbrook), iii, 90, 91

Lanie, de, see Delaune

Lant, iii, I I 8, 120

Lanthony, man. (Arlesey), ii, 203, 262,263

Lanthony Henlow, see Henlow Lanthony

Lanthony Priory (Glouc.), ii, 155 , 263,28 I, 285 ; mills, ii, 283

Lanti vase, iii, 460

Lanvaley, Gunnora de, ii, 243 Hadwisa de, iii, I9o; Will. de, ii, 243 ; iii, I90

Lark-catching ind., iii, 36

Latimer, bar., ii, $203 n$; iii, I 4

Latimer, Eliz., Lady, ii, 203, 238 iii, 13; Lds., iii, I 2, 77, 96 John, iii, 46, 235, $33^{\circ}$; Rich., ii, 203 ; Will., iii, I3

Latimer (Latymer), Alice, ii, 238, $247,248,250$; iii, I93, I94, I98 $n$; Edith, iii, II6; Eliz., ii, 203, 2 II $, 247,256,275$; iii, I3, I14, 216: Nich. le, ii, 247; iii, I94; Will. le, i, 382 ; ii, 203, 2 II, $238,239,247,250,256,257$, 275 ; iii, 3, 21, 96, 194; fam., ii, 235 ; iii, 181, 21 2, 215,383

Latton, John, iii, I03

Lauderdale, earl of, ii, 53

Launcelayns, man., see Wood End, Cople

Launcelyn, Anne, iii, 239, 24I : John, iii, 24I ; Marg., iii, 239, 24I ; Rich., iii, 239: Walt., iii, 239, 247

Laundres, Emery de, iii, 59; Rob. de, iii, 59 ; Steph. de, iii, 59

Laurence, archd. of Beds, i, 31 3

Laurence (Lawrence), Anne, iii, 38I : Edw., ii, 28I $n$; iii, 67 : Geoff., iii, 67 ; Hen., iii, 67 ; Jane, ii, 28I $n$; Joan, ii, 305 : Joanna, ii, 305 ; John, ii, $224 n$; iii, 381, 392; Marg., ii, 305: Nich. iii, 67 ; Rich., ii, 358 ; iii, 67 ; Will., iii, 158

Lau rence Place, see Sokes, man.

Lavendon (Bucks), iii, 3I, 63, II 7

Lawe, Ant., iii, 254

Lawford, Dr. Edw., i, I87; Edw. iii, 416
Lawley, Agnes, ii, $352 n$; Thos., ii, $295,35^{2}, 35^{8}$; Will., ii, $352 n$ Lawrence, man. (Risley), iii, 158 ,

Lawrence, fam., see Lan rence

Lawson, Geo., iii, 319 ; John, iii 106; Nath., iii, 294 ; Nich., iii, 294: Theodosia, iii, I06

Layton, see Leighton Buzzard

Layton, Dr., i, 356, 389, 392

Lea, riv., i, 53, 98, 269; ii, I8n

Leach, Joan, ii, 305 ; John, iii, 60 Judith, iii, 6o ; May, iii, 60 Sam., ii, 305 ; Thos., ii, 305 :
Will, iii, 60

Leachland, Thos., iii, $4 \mathrm{I} 6$

Leader, Frances, Lady, iii, 167 Sir Oliver, iii, I67: Oliver, iii, 204

Leadwell, see Ladywell

Leagrave, ii, 306, 348-50, 374 Brit. coins, i, I 73, I 74 ; ii, 348 chaps., ii, 374; kilns, ii, 2 neolithic rem., $i, 164,166$; ii, $34^{8}$; Rom.-Brit. rem., ii, 8

Leagrave Marsh, i, 269

Leaper, Kath., ii, 342 : Peregrine, ii, 342 ; Sim., ii, 342 ; Thos., ii, 342

Leather, Marg., see Harper, Marg.

Leather-dressing, iii, 64,69

Lecton, see Leighton Buzzard

Ledet (Ledit), Christina, i, 36r ii, 237, 247, 250; Margery, i 36I $n$; ii, 237, 247; iii, 253 Wischard (Wiscard, Wyschard), i, 36I, 364; ii, 237, 247; iii, I95 253

Lee (Podington), ii, 326

Lee, Anne, iii, 340 ; Hen., ii, 385 ; John, ii, 377 ; iii, 122, 377 ; Joyce, ii, 362; Kath., iii, 377; Marg. Lady, iii, 340 ; Philip de, ii, 312 Rob., ii, 362 ; Rob. C., ii, 347 Rog., ii, 376 ; iii, 443 ; Sir Rich., iii, 237, 339, 340 ; Thos., ii, 69 : Will., ii, 385 ; 一, iii, 22 ; see also Leigh

Lee-Antonie, Will., iii, 92

Leedare, Marg., see Harper, Marg.

Leeds, dk: of, iii, 453

Lefstan, ii, 34I $n$

Lega, par., see Thurleigh

Lega, fam., see Leigh

Leget, John, i, 399

Legh, Legha, see Leigh

Leghton, see Leighton Buzzard

Lehton, see Leighton Buzzard

Leicester, diocese, ii, 18

Leicester, hon., iii, I 44, 23I, 294

Leicester, Parnel, ct ss. of, iii, 293 earls of, iii, I 44 ; Rob., ii, 232 iii, 246, 294; Sim. de Montfort. ii, $35 \mathrm{I}$; iii, 439

Leicester, Will, iii. 377

Leigh, Lady Mary, iii, $404,429 n$; Thos., lord, i, $34 \mathrm{I}$; ii, 44,54 iii, $277,401,402,404,408,41 \mathrm{I}$, $4 I_{4}, 429 n$

Leigh (Lega, Legh, Legha, Leya, Leye), Agnes de, $i, 385$; Alicia, iii, 4II : Andr. de la, ii, I05, 326 Anne (Anna), iii, 302, 4II, 415 Barth., iii, IO4; Basile de la, i, 389 ; Chas., ii, 59,62 ; iii, 277 4II, 4I5, 4I6; Dionisia, iii, I05 Eliz., iii, 4II ; Gerard, ii, 353 Gerinus, iii, 51, 54 ; Goddard, iii, 292 ; Hen., iii, 5I ; Hugh de (la) ii, 326 ; iii, 85, 104, Io 8 ; Sir John, iii, 404; John (de la), iii, 2 I, IO 4 105, 147, 277, 334, $41 \mathrm{I}$; John G., ii, 353: John S., ii, 353,356 , 366: Lewis, iii, 2I, 277, 335 Lucy, iii, 277 ; Mary, iii, 292-5 n,
Leigh (cont.)

4 II ; Nichola, iii, IO4 ; Rog. de la, ii, 253 ; Sim. (de la), iii, 24 , 104 ; Steph. (la), iii, 85,87 , I04 108 ; Sir Thos., see Leigh, Thos. Ld. ; Thos., ii, I58; iii, 21, 277 , 300,302 ; Ursula, iii, 411 ; Will., iii, $24,104,146$; Dr. 一, i, 363 368 ; fam. iii, 82 ; see also Lee

Leighton, Baldwyn, ii, 286; Rog. de, iii, $356,357,35^{8}$

Leighton Buzzard, i, 319, 324, 327 349 ; ii, $44,45 n, 47,54,55,62$, $67,71,102,114,116 n, 133,189$, I 9o, 348,349 ; iii, $320,336,367$. $368,383,399$; adv. i, 3I 7, 34r; iii, 414: A.S. rem., i, 176,181 , 187,189 ; bridge, iii, 402 ; chant. iii, 415 ; chaps., i, 346 ; char., iii, 368,415 ; chs., i, 3II, 312, $324 n$ 329; iii, 400, 402, 409-4I4 Danes in, iii, 4 or ; fire, iii, 402 ; gild, iii, 415: Hermitage, the iii, 409 ; hound money, ii, $143 n$. inns, iii, 399, 400, 415; liberty, iii, 337: mans., i, 319 $n, 403$ iii, IOO $n, 337,402-9$; market cross, ii, I00 ; iii, 399 ; mkts. and fairs, ii, 76,88 ; iii, 401 ; mills, iii, $400,408,409$; Nonconf., ii, 375 ; iii, 4 I 4 ; place-names, iii, 402,408 prebendal estate, iii, 408; prehist. rem., $i, 145,162,173,174$; racing, ii, Igo, IgI ; rectory, $i$ 341 ; Rom. rem., ii, I, 8, 9; iii, 402 ; sand ind., ii, $\mathrm{II}_{7}$; schs., ii, I83; iii, 416; wheat-growing experiments, ii, I34 ; witchcraft, ii, 100 : iii, 402

Leighton Heath, i, 18

Leighton prebendal (rectorial), man., iil, 408

Leith, Rev. Alex., iii, 33 ; Martha, iii, 32, 33

Leiton, see Leighton Buzzard

Lekeburn, Pet, de, iii, I72

Le Mesurier, Rev. H. C., ii, I 74

Lenborough (Bucks), ii, i $8 n, 349 n$ iii, 4 OI

Lenham, Isolda de, iii, 452; John de, iii, 452

Lennox, Marg., ctss. of, ii, 339

Lenton Priory, i, 315 $n$; ; ii, 288, 292,293 ; iii, 63,80

L'Enveise (Enveyse), Agatha (Alice), iii, 181, 182; Jordan, iii, I25, I8I; Walt., iii, 181; Will. iii, 125

Leofnoth, ii, 326

Leofwin (Leofwine, Lewin), i, $296 n$, 347 ; ii, $21 n, 204,234,278,288$; iii, $70,24^{8}$

Leominster, Sophia, Lady, iii, 453 Thos., Ld., iii, 453; Will., Ld. iii, 452

Lepidoptera, i, 78-88

Lepine, Maria, iii, 444

Lestrange (Lestraunge), Geo., Ld., iii, 140

Lestrange (Lestraunge, L'Strange, De Extraneis), Joan, iii, II9 Matilda, ii, $268 n$; iii, I6 $n$ Maud, ii, $300 n$; iii, 44, 215, 329 Rob., i, $379 n$; iii, 137,150 Sir Rog., iii, 44; Rog., ii, $300 n$ 339,341 ; iii, 12, 52, 97, 21 5, 262, 329 ; Thos., iii, I 50

Lessy, Phil., iii, 294 n

Lester and Pack, bell-makcrs, ii 338 ; iii, 25

Lestone, see Leighton Buzzard

Lethers, Marg., see Harper, Marg. :

Rich., ii, I60

Lethom, Will. de, i, 401

Letitia, ii, 376 


\section{A HISTORY OF BEDFORDSHIRE}

Leuiet, see Leviet

Leutton, see Luton

Levenot, iii, 70, 113,118

Leventhorpe, Ann, ii, 289 ; Eliz.. ii, 289, 29I; Geo., ii, 289; Joan, ii, 289 ; John, ii, 289 ; Thos., ii, 289,29

Leverington, Ad. of, i, $3^{87}$

Leveson-Gower, Gertrude, ii, $62 n$

Leveva, iii, 338

Leviet (Leuiet), i, 312n ; pricst, iii, 37,107

Levinz, Sir Creswell, iii, 150, I59; Creswell, iii, 83, 105; Will., iii, 105

Levret, iii, I 20

Levric, iii, 105

Lewelyck, Denise, i, 360

Lewen, Rich., iii, I 46

Lewes (Pegsclon), ii, 295 x

Lewin, sec Leofwin

Lewis, Jas., iii, 446 ; Will. V., iii,

Lewknor, Cath., iii, I95; Eleanor, iii, 194, I95; Rog., iii, I94, 195 I.cwsey, man. (Luton), ii, 350, 363

Leyburne, Eleanor de, ii, 351, 353, 354 ; Ida, if, 230, 232; Idonea de, ii, 230 n; Rog., ii, 231, 232, 351

Leyghton, see Leighton Buzzard

Leyham, Isabel de, ii, 207; John de, ii, 208; Matth., de, ii, 207 ; Oliver de, ii, $207 n$; Pet. de, ii, 207,208

Lichens (Lichenes), i, 60-1

Lichfield and Coventry, bps. of, i, 343 ; ii, 224

Lidlington, ii, 66 : iii, 4, 267, 305, 422 ; adv., i, 32 I $n, 322$; iii, 307 char., iii, 307 ; ch., $i, 313,322$; iii, $305,306,307$; crown property, iii, 271; farms, ii, 131, 134; iii, 305 ; inclosures, ii, 97 ; inds., iii, 305; man., i, 312; iii, 272, 305, 306: Nonconf., iii, 305, 307; rents, ii, 132 ; schs., ii, 183 ; iii, 307 ; spring, iii, 308 ; vicarage, i, $329 n$; iii, 307 ; workhouse, ii, 104

Lidlington, Agnes dc, iii, 325 ; Thos., iii, 307 ; Will. of, i, $3^{87}$

Lightgrave, see Leagrave

Lightgrave, man., see Woodcroft

Lilincletone, see Lidlington

Lillingestan, see Lidlington

Lilly, Hen., iii, I 50

Limbury, i, 173, 175, 269, $269 n$, 280 ; ii, $18 n, 306,308,348,349$, $3624,374,384$; iii, 401 ; mans., ii, $350,360,366$; mill, ii, 360 ; Nonconf., ii, 374 ; Rom. rem., ii, 8

Limbury (Lymbury), Joan de, ii, 360 ; Phil. de, ii, 360 ; Ralph de, i, 329 ; Rich. de, ii, 360

Limersey Grange, see Lymbotsey

Limestone quarries, iii, $96,44^{8}$

Lincelade (Bucks), iii, 12, 14, 46

Lincoln, ii, 24, 33; ii, 153

Lincoln, cathedral ch. of, i, $376 n$, 383 ; ii, 154 ; iii, $21,346,408$; endowment, i, 3II; ii, 210 ; library, ii, 152; prebends, ii, $2 \mathrm{I}_{4} ; \mathrm{iii}, 284 n$

Lincoln, diocese of, $i, 346,347,379$

Lincoln, archds, of, ii, I54; iii, 359

Lincoln, bps. of, i, 3II, $315 n, 321 n$, $327,328,330,339,340 n, 354$, $358,360,361,375,378,379$, $387 n, 396,400 ;$ ii, $38,88,97$, $149,154,178,206,208,210,212$, $2 \mathrm{I}_{4}, 230 \mathrm{n}, 252,253,258,296$, $312,319,343,356,374,380$; iii, $22,29,37,69,116,133,155$,
Lincoln, bps. of (cont.)

$159,185 n, 250,264,272,284$, $334,342,348,349,359,364,374 n$, $398,408,414,43 I, 432,437$ i Alexander, ii, 2 Io; Barlow, i, $339 n$; Beaufort, i, 328, 397 ; iii, 414; Buckingham, i, 330,355 375,379 ; ii, 178,296 ; Burg. hersh, i, 36o, 375 ; ii, I49, 297 iii, 49 ; Chesney, $i, 31_{3}$; Dalderby, i, $359,362,375,383,389,395,397$, $398,40 I$; ii, 209 ; Gravesend, i, $317,322,35 \mathrm{I}, 354,374$; iii, 148 , 334: Grey, i, 329, 360, 376, 380 383 ; ii, 179 ; iii, 414 ; Grossetête, i, $317,322,366,374,379,382$, $383,385,400 n, 401$; iii, 208 ; Gynwell, i, 355, 362, 375, 389 : Holbeche, ii, 210; Hugh of Avalon, i, 313, 388 ; ii, 150, 2 I2 $299 n, 312,335$; iii, 142; Hugh de Wells, i, 316, $317 n, 322,372$, 396 ; ii, 332 , iii, 208, 214, 304, $334,348,364,398,414$; Longland, $i, 356,376,383,384,396$; Montaigne, i, $339 n$; Remigius, i, $311,312,347 n$; ii, I53, I54, $275 n, 276$; iii, 22, 4I4 : Repingdon, i, $355,356 n, 383,3^{88 n}$; ii, 384 ; iii, 170 ; Rotherham, ii, 352; iii, 299; Sutton, i, $317 n$, $322,323,325 n, 354 n, 359,374$ $375,389,395,398,401 ;$ ii, 212

Lincoln, deans of, i, 320 ; iii, 22 , $384 n$

Lincoln, Edw., earl of, iii, 94, 382

Lincoln, Alured of, iii, 119,122

Lincoln College (Oxf.), ii, 176 178

Lindon, hon., iii, 247

Linford (Bucks), iii, 271

Linley, John de, iii, 456

Linslade (Bucks), i, 392 ; iii, 399 $400,401,415$; man., iii, $405 n$; rectory, $i, 393$

Lisle (de Insula, L'Isle, Ly?e), Alice de, ii, 267; John (de), i, 393 ; ii, $224 n, 267$; iii, 285, 395; Maud (de), ii, 267: Ralph de, ii 2Io, 2 II, 2I2; Rob. (de), ii, 267 , 268; iii, 7o, 285, 395; Rose, sec Wahull; Warine de, ii, 267, 268 ; Sir Will. de, ii, 267 ; Will. de, iii, 122

Lismore, Rob., bp. of, ii, 379, 380 ; iii, 43 I

Lister, Lady, iii, 203

Litchfield, Hen., iii, 380 n

Litgrave, see Leagrave

Little, H., ii, $25^{\circ}$

Little England's, field (Dunstable), iii, $35^{\circ}$

Littlewood Farm (Clapham), iii, 28

Littleworth (Wilshamstead), iii, 326

Litton (Litten, Lytton), Agnes, iii, 220: Anne, ii, 30I: John R., ii, 301: Judith, ii, 301 ; Rowland, ii, 239, 241, 301 ; iii, 28r ; Thos., iii, I46; Will., ii, 301 ; iii, 220

Livesay, Eliz., tii, 83,87 ; Jane, iii, 84; John, iii, 83; Maj.-Gen. John, iii, 83, I19; Paradine, iii 83 ; St. Andr., iii, 83 ; Will., iii, 83

Livesey (Norfolk), man., i, 360

Livius, Geo., iii, 21, 304

Liz, Muriel le, iii, 6o; Robt. le, iii, 60

Llanthony, see Lanthony

Lloyd, Will, iii, 184

Loccles (Locels), Will. dc, ii, 344 , 381,383

Lock, Nary, ii, 363

Lockington, Mary, iii, 367,368

Lockwood, fam., iii, 57
Lodge, Alice, iii, 430; Ellen, iii, 430 ; Hen., ii, 40 ; iii, $43^{\circ}$; Mich., iii, $43^{\circ}$

Lodington (Lodynton), John, iii, 273; Thos., iii, 423

Loitone, see Luton

Lollards, the, i, 330

Lomax, Caleb, iii, 332 ; F. G., ii, 218 ; Joshua, iii, 332 ; Ruth, iii, 332 ; $\frac{}{1 i}, 216,257$

Londe, Walt. de la, iii, 137

London, ii, $365,370,392$; ii, 135 , I63: Beds property, ii, 3ro; iii, $253,285,292,321,326,332$; riot, ii, 30

London, bps. of, i, 329, 332, 400 ; ii, 38,320 ; iii, 194,349

London, ld. mayor of, ii, I58 ; iii, 25, 372

London, Augustine, i, 365 ; Dan. T., i, 363, 364; John, of, i, 377 ; Sim. de, iii, 16

London End (Kèysoe), iii, 136

London End (Milton Ernest), iii, 144

Lone Close (Goldington), iii, $208 n$ Loney, Sir Rich., iii, 286

Long (Longe), Cecil, iii, 301 : Dorothy, iii, 302, 376; Eliz., ii, 330 ; iii, 302,376 ; Hen., ii, 330 ; iii, 230, 376 ; Marg., Lady, iii, 318 ; Sir Rich., ii, 277, 330, 379 ; iii, 23, 213, 240,260, 264, 302, $315,318,324,332,376,437$; Steph., ii, I55; Thos., ii, 374; Sir Will., iii, 303, 305 ; Will., iii, 301

Longbridge (Warws), i, $18 \mathrm{I}$

Longdon, Hen., ii, $24^{8}$

Longe, see Long

Longespee, Will, see Salisbury, earl of

Longeville, see Longueville

Longford, ctss. of, ii, I 8 r $n$

Longland, bp., see Lincoln, bps. of

Longley plantation (Podington), iii, 80

Longney (Gloucs), iii, 25 I

Long S towe (Cambs.), iii, 4 I 5

Long Tag Wood (Stevington), iii, 100

I.ongueville, Anne, ii, 290, $295 n$; Chas. G., ii, 290; Frances, ii, 295; Grey, ii, 290, 295 ; Hen., ii, $290,295 n$; Isabella de, iii, 189 : John de, iii, 187, r99; Lucy, ii, 290 ; Marg. (de), ii, 295 ; iii, 184 , 187 : Sir Michael G., ii, 290 ; iii, $228 n, 232$; Susan, ii, 290; iii, 232 ; Susanna, Lady, iii, $228 n$

Longworth, John, ii, I66, 167, I68

Lord, John, ii, 277 : Obadiah, ii, 385

Lord lieut., ii, 56, 59, 6I, $62 n$, I 75

Loring (Lorenge, Loryng), Dav., iii, 33I; Isabel, iii, 89; John (de), ii, 252, $356 n, 357$; Marg. iii, $346,44^{\circ}$; Sir Nigel (Neel), i, 325,329 ; ii, 353 ; iii, $89,346,348$, 349,440 ; Pet. (de), ii, 318 ; iii, $89,346,347,349,367$ : Rob., iii, 345 ; Rog., i, 37 I $n$; iii, $89,34^{8}$; Will, ii, $318,353,356 n, 357$

Lorings, man. (Sharnbrook), iii, 89

Lorraine, Alb. of, ii, 22 ; iii, 89, 329, $332,345,34^{6}$

Loryng, see Loring

Lotrell, Hugh, ii, 227

Lovat, riv., see Ouze

Loveday, Anne, i, $38_{4}$,

Lovelace, Anne, Lady, iii, 44 I

Lovell (Lovel), Fran., ii, 2 Io ; H., iii, 278; Sir John, ii, 3r6; John, iii, 422 ; Pet. H., ii, 2 lo; Phil., iii, 422 : Will., iii, 231 


\section{INDEX}

Lovells (Lovells Bury), man. (Potsgrove), iii, 422

Lovett (Lovet), Rob., ii, 29I ; iii, 283,339 ; Will., iii, $284,286,395$

Lowe, Fran., iii, 322 ; John, iii, 396 ; Will. D., iii, I33

Lower Berry End (Eversholt), iii, 375

Lower End Farm (Lidlington), iii, 305

Lower Rads End (Eversholt), iii, 375

Lowick (Luffewyche) (Northants), iii, $92 n$

Lowndes (Lownde), Eliz., iii, 330 : Kath., iii, 285; Marg., iii, 285 ; Rich., iii, 330 : Thos., iii, 285 ; Rev. Will,, iii, 330: Will,, iii, $33^{\circ}$; Will. L., iii, $33^{\circ}$

Lubenham, Rob. of, i, 386, 402

Lucas, Ladies, iii, 66, 228; Amabel, see Grey of Wrest, ctss. of ; Anne, ii, 327 : Ld., ii, I42, I97, 200, $325,327,334$; iii, 64 ; see also Grey, Lds., and Kent, dks., etc.

Lucas, Rob., ii, 270

Lucas and Dingwall, Ld., ii, 283 , $290,305,322,323,325,332$, $333,337,345,377,379,380$; iii, $5 \bar{z}, 65,68$; Auberon Herbert, Ld., ii, 328

Lucy (Luci), Alice, iii, 224; Anne, iii, 427, 429; Sir Edm., iii, 230 ; Edm., iii, 5o ; Eleanor, Lady, iii, 230,248 ; Eleanor de, ii, 354, 422 ; Eliz., iii, 5o; Emery de, ii, 354 ; Fran., iii, 330 ; Geoff. de, ii, $30 n$, 354 ; iii, 405, 409, 422 ; Geo., ii, $204 n$; Joan, ii, $204 n$; John, iii, 286; Juliana de, iii, 422 ; Marg., ii, 354, 429; Maud, iii, 422; Rebecca, iii, 330 ; Reg. de, ii, 354 ; Sir Rich., iii, $181 n$; Rich. (de), ii, $26 n$; iii, 330 ; Sir Thos., iii, 224, 330 ; Thos., iii, 5o, 2II, 224: Walt. de, ii, 354 : Sir Will., iii, 405, 422 ; Will. de, ii, 354; iii, 50, 427, 429

Ludgershall (Bucks), i, 400; iii, I $75,176 n$

Ludlow, Ld., ii, 187

Ludlow, fam., ii, 66

Ludsop, John, ii, 36

Luffewyche (Northants), see Lowick

Lufwyk, Rob. de, i, $382 n$

Luke, St., relics of, $i, 400$

Luke, Anne, ii, 262 ; Cecily (Cecyle) Lady, iii, I93, 241 ; Eliz., ii, 216 , 340 ; Frances, iii, 195 ; Sir John, iii, 193, 239; John, ii, 262, 263 . 282 ; iii, 193, 198; Sir Nich., ii, 216 ; iii, $158 n, 193,239,241$. Nich., ii, 262, 282, 342, 382 ; iii, 29, 146, 193, 197, 209, 212 ; Sir Oliver, ii, $42,44,282,339$, 340,342 ; iii, 29, 193, 239 : Oliver, ii, $262,282,34^{\circ}, 34^{I} n$, 382 ; iii, 29, 195; Paul, ii, 235 . iii, I98; Sir Sam., ii, 4I, 42, 44 $45,46,47,48,49,54,55,340$ $343 n$; iii, 227, 238, 239, 404 456 ; Sir Walt., iii, $239,24 \mathrm{I}$ Walt., ii, 40 ; iii, I97; Will., iii, I97; fam., ii, 44,60 ; iii, 238 , 342,343

Lunde, la, wood (Little Staughton), iii, I65

Lupton, Rog., ii, 157

Lusignan, Guy de, ii, 29

Luthmere, Thos., iii, I 25

Lutlingeton, Lutlyngton, see Lidlington

Luton, ii, I $8,67,100, I_{4}, 120$, I47, 295, 305, 306, 3I 4, 32I, 326,
$333,338,342,344,348-75$; iii, I0, 105, $317 n, 325$; adv., i, $314,317,336,34 I$; ii, 373 ; iii, 393 ; almshouses, ii, 375 ; chant. ii, 374,381 ; char., ii, 374 ; iii, 238 ; ch., i, 3IO, 3II, 3I 4, 3I 5 n, $324,346,372 n ; 400$; ii, 349 , $356,368,381$; courts, ii, 308 366 ; deanery, i, 347 ; fairs and mkts., ii, $76,88,349,351,352$ $353 n, 360$; fire, ii, 85,366 gilds, i, 330 ; ii, 92, I49, 374 $38 \mathrm{I}$; hosps., i, $349,399,400$ ii, 375 ; hound money, ii, $143 n$. inds., ii, 109, I19, I21, 122, 126 I27, 349,350 ; inns, ii, 348 mans., i, 315 $n$; ii, 143, 306-8, $349-60,362 n, 364,366,374,383$ mills, i, 311 ; ii, $351,357,366$ neolithic impls., i, 174; Nonconf., i, 344 ; ii, 374 , 375 ; placenames, ii, 348 ; plait halls, ii, II9, I2I ; Rom. Cath., ii, 374; Rom. coins, ii, 9 ; schs., ii, I49, 180, I83, 375; vicar, $i, 316,324 n$, $327 n, 333,395 n, 400$; Witan at, ii, 350 n

Luton (Leutton, Lutton), Ad. of, i, 370; Alice de, ii, 357; John, iii, 233; Mary, iii, 312; Maud of, i, 360 ; Rob. de, ii, $276 n$; Rog. de, ii, 357

Luton Castle, ii, 350

Luton Downs, ii, I 98

Luton Hoo, man., ii, 6r, 119, 197 , $257,348,350,353 n, 355,357$, $36 \mathrm{r}, 365,373,375$; mill, ii, 366 : Rom. coins, ii, 9

Luton Hoo Park, ii, $147,348,355$, 360

Lutune, see Luton

Luvholt, Godf. de, ii, 376 ; Mabel de, ii, 376

Lycett, Inskip \& Co., ii, 262, 263

Lydall, Rich., iii, 26

Lyde, Hen., iii, 198

Lye, Rev. Thos., ii, 375

Lyencourt, Will. de, i, 403,404

Lygetune, see Leighton Buzzard and Luton

Lygon, Mary, iii, 430 ; Rich., iii, $43^{\circ}$ see Lisle

Lylebone, Sir John de, ii, 378 ; Sibyl de, ii, 378

Lymbotsey (Limersey) Grange (Ampthill), iii, 273

Lymbotseye, John de, iii, 292

Lymbury, see Limbury

Lyner, Godf. de, iii, $3^{14}$

Lyne-Stephens, Mrs., ii, 270

Lynford mill, ii, 283

Lynn (Norfolk), ii, 9

Lynom, Thos., iii, 92

Lysons, Mary, iii, 28

Lytlington, see Lidlington

Lytton, see Litton

Mabel, Abbess of Elstow, i, 355

Macan, -, ii, 189, 190

McDowall, - ii, 177

Machell, John, ii, I58; Capt., ii, I94

Machinery manufacture, ii, 125-7

Mackenzie, Sir Geo. S., ii, 197, 25 I

Maclear, Basil, ii, 200

Macnamara, Anne, ii, 318 ; iii, 372 Arth., ii, 318 ; iii, 372 ; John, ii, 318 ; iii, 372,455 ; Sophia, iii, 400

MacQueen, Dr. Malcolm, iii, 293 322 ; T. Potter, iii, 322 ; Dr., ii, 133

Madan Castle, see Maiden Bower
Maddison, Col., iii, $3^{61}$

Madin-boure, see Maiden Bower

Madning-bowre, see Maiden Bower

Maenfinin, ii, 23

Maggotts Moor (Ampthill), iii, 274

Magiovinum (Bucks), see Brickhill, Little

Magniac, Chas., iii, 90, 92, 94, 95 , I40, I 42; Hollingworth, ii, I 88 iii, 92 ; H. R., iii, I42; Vernon, iii, 94,95

Magpie Hall Farm (Marston More taine), iii, 313

Maidbury, man. (Elstow), iii, 28I, 282

Maidbury (Maydebury), Thos., iii, 282

Maiden Bower (Dunstable), Rom Brit. rem., i, I60, $163,164, \mathbf{1 6 6}$ I69, I70, I74, 269, 294 ; ii, 2, 7,9 ; iii, 390

Maidwell, Cutts, iii, 57 : Godf., ii 345 ; Thos., iii, 57 ; Will. L., iii,

57
Maister, see Master

Makehams Close (Steppingley), iii 325

Malcolm IV, king of Scotland, i, 361,$388 ;$ iii, 23,65

Maldon, battle of, ii, 20

Malens, man., see Maryons

Malens, fam., see Malyns

Malet, Hugh, i, 370

Malherbe, Geoff., iii, 293 ; Joan, iii 292 ; John, i, 401; iii, 52, 292 383 ; Lucy, iii, 384 ; Pain, iii, 52 293 ; Rob., iii, 29I, 383, 385 ; Will., iii, $44 n, 52$

Malory, Alice, ii, 286 ; Anketin, iii 162: Bertram, ii, 286: Eleanor, ii, 286 ; Fran., iii, 162, I64; John ii, 286 ; Pet., iii, I62; Ralph, iii, I62; Rob., ii, 286 ; Sim., ii, 286 Thos., iii, 162 ; Sir Will., iii, I62: Will., iii, I6

Malyns (Malens), John, iii, 183,211 $212,229,231$; Thos., iii, 183, 231 Mammals, i, 138-43

Man, see Mann

Mancels Grove (Turvey), iii, II 4

Manchester, Chas., dk. of, iii, I69 ; Hen., earl of, iii, 168

Mandeville, Geoff. de, i, 378 n, 390 ii, 25; see also Essex, earl of Hen., iii, I 71 ; Isabel (de), iii, 70: Rohese de, see Beauchamp Walt. de, ii, 354; Will. de, iii, I7I; see also Essex, earl of

Manepeny, Will., i, 370

Maney, Hugh, ii, 332, 333

Mangehoo, man. (Marston Moretaine), iii, 3II

Maningham, see Manningham

Manley, John, iii, 326 ; Pet. de, iii 223, 224

Mann (Man, Manne), John, ii, $327 n$ 378 ; iii, 408; Mary, iii, 408 Will., iii, 408 ; Mrs., iii, 383,384 Manning, Rev. - , iii, I62

Manningham (Maningham), Sir John, ii, 220, 257; John, ii, 219; iii, 231; Kath., ii, 257 ; Sir Will. ii, 257

Manor Farm (Goldington), iii, 202

Manshead, hund. of, ii, 95, I14, II 6 ; iii, 70 , 336, 337 ; assessment, iii, 39I

Mantell (Mauntel), Hen., iii, 366 ;

Marg., iii, 456; Thos., iii, 456: Walt., iii, $45^{6}$

Manyngham, see Manningham

Marborowe, Hen., iii, I55: Thos., iii, 155; sec also Marlborough

Marbury (Marbery), Edw., ii, 307 : Eliz., iii, 255: John, iii, 255; 


\section{A HISTORY OF BEDFORDSHIRE}

Marbury (cont.)

Lewis, ii, 307 ; Thos., iii, 255, 261, 393

March, earls of, ii, 191, 192, 328 : Edm., ii, 328; Rog., ii, 33, 327 ; iii, 229, $28 \mathrm{I}$

Marche, Will. de la, iii, 286; see also Marsh

Mareis, Adeliza (de), i, 392 ; ii, 267 Walt. de, ii, 267; see also Marsh

Mareschal, see Marsha

Margets, Thos., ii, 55, 212

Market gardening, i, 39 ; ii, 118 , т30, 138-9, 213; iii, 181, 189, 2 I $8,242,256,284,369$

Markets and Fairs, ii, $46,87,88$, IO2, I O5, I 2 I, I $40,237,239,242$ $26 \mathrm{I}, 263,266,268,330,34^{8}, 349$, $35 \mathrm{I}, 352,353 n, 360,385$; iii, I6, $17,21,35,64,69,73,142,199$, $229,246,254,258,268,269,27 \mathrm{I}$ $276,28 \mathrm{I}, 3 \mathrm{II}, 3 \mathrm{I} 7,34^{\mathrm{O}}, 355,36 \mathrm{I}$, $362,363,401,44 \mathrm{I}, 453,459$

Market Street (Herts), see Markyate Street (Herts)

Markham, Ad., ii, $356 n$; Alice iii, 85; Frances, ii, 355, 356 ; Gervase, i, 374, 377 ; iii, 36I ; John, ii, $355,356,357$; iii, 85 , 285 ; Kath., iii, 85 ; Thos., iii, 85 ; Will., ii, 355,356 ; iii, 395 , 398

Markyate, Christine of, i, 350; Rog. of, i, 350

Markyate Cell (Caddington), ii, 3 I 7

Markyate Priory, i, $315 n, 316 n$, $349,35 \mathrm{I}, 35^{8-61}, 395$; ii, 316, $347,383,387$

Markyate (Market) Street (Herts), ii, $71,92,314,316-20,377$, 380 ; iii, 337,455 ; A.S. rem., i, 146 ; char., ii, 320 ; ch., ii, 319 ; Five Horse Shoes Inn, ii, 318; man., ii, 316, 317; Nonconf., ii, 320 ; Rom. rem., ii, 3, 7 ; sch., ii, 3 I 7,320

Marlborough, Jane, ctss. of, ii, 347 , 382 ; dchss. of, iii, 211,240 ; Sarah, dchss. of, iii, I04, 203 , 213, 214, 224, 264, 306, 309; earl of, iii, 4 ; Jas., earl of, ii, 347,382

Marlborough, Marg., iii, 98; Thos. ii, 295n; iii, 98; see also Marborowe

Marlie, Will., iii, 363

Marmyon, Sir Will., iii, I62

Marsh (Marshe), Blandina, iii, 367, 368, 416 ; Dan., iii, 368 ; Hen., ii, 316 ; John, iii, 60 ; Rich., ii, 316; iii, 332 ; Rotherham, ii, 316 ; Thos., ii, 316 ; Ursula, iii, 60

Marshal (Mareschal, Marshall), Alina, iii, 426; Anselm, see Pembroke, earl of ; Edm., iii, 442 Ela, iii, 426 ; Eliz., iii, 98, 445 Hawisia, iii, 426; John (le), iii, I47, 329, 39I, 426; Maud, ii, 220 ; iii, 329,439 ; Rog., i, 382 ; iii, 329: Sarah, iii, 329; Thos. i, $325,373,375,376,377$; ii, I78; iii, 98, 329 ; Walt., ii, $8 \mathrm{I}$ 220,267 ; Will., ii, 27, 34I, 350 , 35 I $n, 362 n, 366$; iii, 426 ; see also Pembroke, earl of : - ii, 382

Marsh Wood (Renhold), iii, 2I 5

Marsiliaceae, $i, 56$

Marsland, Clem., iii, $25 \mathrm{I}$

Marston (Northants), i, I82

Marston, North (Bucks), ch., i, 37 I $n, 373,376 n$

Marston Moretaine (Morteyne), i, 324 ; ii, 66, II5, 250, 324 ; iii, 11 , $13,132,267,277,298,307^{-1} 3$ 32 I, 333 ; adv., iii, 313 ; char., iii, 3I3; ch., i, 3I 8 ; iii, 308, 3II-I3
Marston Moretaine (cont.) farms in, iij, 307; inclosures, ii 97: mans., iii, 309; mkts. and fairs, ii, 88 ; iii, 3 I1 : Nonconf. iii, 308, 313; schs., ii, 183

Marston Park Farm (Marston Moretaine), iii, 308

Marston Pillinge (Marston Moretaine), iii, 308, 313

Marston Thrift (Marston Moretaine), ii, I 87 ; iii, 308

Marsworth (Bucks), ch., i, 382-4

Martel, Osbert, iii, 370

Martin, papal nuncio, ii, 29

Martyn (Marten, Martin), Dr. Edw. i, 339-40, 343: Eliz., iii, 330 Eulalia, iii, I57 $n$; Gilb., iii 203 ; John, ii, 227; iii, 145 ; I 57,220

Marvin, Rev. -, ii, 347

Marwen, see Morwen

Mary, princess (dau. of Edw. I) i, 404

Mary 1, queen (of England), ii, 377

Mary, queen (of Scots), ii, I I9, 349

Maryon, Isabel, iii, 240; Thos., iii, 240

Maryons (Malens), man., (Cople), iii, 240

Mason, Bartlett, ii, 280

Massey, Eliz., Lady, iii, 443 ; J ane, iii, 402 ; Sir Walt., iii, 443

Massingberd, -, iii, $422 n$

Master (Maister), Marg., iii, I45 ; Streynsham, iii, 145i Thos., ii 310 ; iii, 216 ; Will., ii, 334

Mather, Will., iii, 9

Mathers, John, iii, 3I

Matilda, see Maud

Mat industry, see Rush-matting ind.

Matthew (Mathew), John, iii, 333 Mary, iii, 19I; Sarah, iii, 333 Will., iii, 333

Matthews, Millicent, iii, 368

Mattock, Eliz., ii, 363

Maud (Matilda), ctss., ii, 237; iii, 302,304

Mauder, Harry, iii, 183

Mauduit, Arth. iii, 443; Eliz., iii, 443: Maria, iii, 443 ; Sarah, iii 443 ; Will., see Warwick, ear] of

Maulac, Lavaric de, ii, 27

Maulaye, Alice, iii, 233; Rich., iii, 233

Maulden (Meldone), i, 313, 353-4, 356 ; ii, $66,115,135,32 \mathrm{I}, 324$; iii, $267,273,275,286,313-316$; adv., iii, 315 ; char., iii, 316 ; ch., i, 3I 5 n, 357; iii, 3I 4, 3I5: inclosure in, ii, 97 ; inns, iii, 313 man., iii, 314; mills, iii, 314; Nonconf., iii, 3I4, 3I6; placenames in, iii, 314 ; poorhouse at, ii, 104 ; sch., ii, 183

Maulden Moor, Rom. rem. at, ii, 9

Maulden Wood, iii, 3 I 3

Maultby, - iii, 4 I 6

Maunsell, Sampson, iii, II 3 ; Will., iii, II3

Mauntel, see Mantell

Mavorn (Maverns, Mavourn, Glintells), man. (Bolnhurst); iii, I26: earth works, $i, 306$; iii, 124

Mawcase Field (Wilden), iii, 226

Mawditt, see Mauduit

Maxey, -, ii, 97

Maxie, Edw., ii, I6r

May, John, iii, I 59

Maydebury, see Maidbury

Maydenford, Pet. of, i, 352

Mayfield Farm-house (Markyate) ii, 3 I 4

Mayhew, Rev. Geo., ii, 206, 265
Mayn, John, iii, 133

Maynard, Will., Ld., ii, 24

Maynard, John, ii, 342, 344, 353 ; iii, I32, I47, 26I, 408

Mays, Rich., ii, 232

Mead (Meade), Ann, Lady, iii, 68 ; Jas., ii, 27 I, 293; John, iii, 449 : Rich., ii, 383 ; iii, 404 ; Rob., iii, 432 ; Rev. T. W., iii, 428 ; Will. iii, 45I; Dr. - , iii, 66

Mears, bell-founders, ii, 218,222 , 236, 24I, 250, 254, 287, 319, 347 380,383 ; iii, II $6,214,255,278$, $295,307,3 \mathrm{I} 5,4 \mathrm{II}, 423,446,454$ Mears \& Stainbank, bell-founders, ii, 229, 245; iii, 116, 26I, 266, $41 \mathrm{I}, 461,462$

Measures, Benj., iii, I 7 I

Medbury Farm (Elstow), iii, 282

Medgate, Jos., iii, 392 ; Thos., iii, 392

Medland, Will., iii, 60

Medlicott, Thos., ii, 28 I

Medmenham, man. (Bucks), i, 366 370

Meech, John, ii, 3 I 7

Meen, Eliz., ii, 215

Meisi], Rob, de, iii, I92

Melchbourne (Melceburne), i, 349 ; ii, 107, II $5, I_{4}^{6}, 197$; iii, $59 n$, 95, I 23, I 32, I 42-143, 154 ; adv. iii, 143; chap., iii, I43; ch., i, 3 I $4 n, 315 n, 3$ I 8,394 ; iii, I 43 ; man., i, 394; iii, I 42 ; mkts. and fairs, ii, 88; iii, I 42 ; mills, iii, 142, I 43 ; priest's stipend, i, 336 ; sch., ii, 183

Melchbourne, Rich. de, iii, I37

Nelchbourne Park, ii, 147; iii, 4I, 142,168

Melchbourne Preceptory, i, 315n, 394; ii, I 47 ; iii, 95, I 42, I 43 , $154,165,198$

Meldone, see Maulden

Melebroc, see Millbrook

Mellents, fam., ii, 2 I $n$

Melton Mowbray, man., iii, 346

Mendham, Rev. John, ii, 325

Mentmore (Bucks), i, 402

Mentmore, Hugh de, iii, 376 ; Miles de, ii, 333

Meppershall (Mepartyshall, Meperbeshale, Mepertesale, Meppersal), ii, $72,113,260,266,270,275$, $276, \quad 288-293,304,305,34 \mathrm{I}$; adv., ii, 292 ; chap., ii, 293 ; Chapel Farm, ii, 288 ; char., ii, 293 ; ch., i, 3 I $5 n, 332 n$; ii, 29I, 296: earthworks, i, 296: ii, 24,288 ; mans., i, $296 n$, 39 I, 393 ; ii, 288 ; moat, ii, 288 ; place-names, ii, 288 ; rectory, ii, 288 ; sch., ii, I83

Meppershall (Meptyshall), Gilb. de, ii, $290,291,293 n$; iii, 63 ; Joan de, ii, 289, 290; iii, 243; John of, ii, $288,289,290,292,293 n$, $296 n, 334$; iii, 243; Kath. de, ii, 289, 292 ; Nich., ii, 275, 289 ; Ranulf of, ii, 289 ; Rob. de, ii, 288, 289, 292 ; iii, 59, 63; Sarah de, ii, 289: Will. de, ii, 288 ; iii, 63

Meptyshall, see Meppershall

Mercer, Col. Algernon, ii, 147 ; iii, 228, 231, 233, 439; Isabella, see Cardun ; Rich., iii, 23I, 233 : Will. Je, iii, IIg

Mercers Co. (London), iii, 347

Merchant Taylors Co. (London), ii, 158, I 59, I60, I6I

Mercia, ii, I8-2I ; bps. of, i, $34^{6}$; earldom of, ii, 20

Mercstun, see Marston Moretaine

Mere, John de, ii, 322 


\section{INDEX}

Merefield, Ralph, ii, 357

Meriet, John de, ii, 306 ; Mary (de), ii, 306, 35I

Merlyn, John, iii, I 54

Merry, Rich., iii, 352 ; Will., iii, 417

Mershtone, see Marston Moretaine

Merston, Jobn de, iii, 3ro; Nigel de, iii, 3ro: Ralf de, iii, 3 Io; Rich. de, iii, 3 Io

Merstone, see Marston Moretaine

Merton Priory (Surrey), i, $37 \mathrm{I} n$; ii, I 54, 291, 305; iii, 29, 374 , 4 I $8 n, 4$ I 9,42 I

Merwe, Maud, iii, 329 ; Walt., iii, 329

Meryng, Idonia de, ii, 29o; John de, ii, 290 ; Thos. de, ii, 290

Meschin, Will., iii, I33

Mesurier, Le, see Le Mesurier

Metcalfe (Metcalf), Chas. J., iii, 219 ; Chris., iii, 206, 209 ; Ellen, iii, 206: Jas, iii, 2 I9; Thos., iii, I 8 ; Will., iii, 2 19; fam., iii, I83, 220

Methodists, i, 345; ii, I 86, 2 I 4, 234 , $242,246,265,299,303,312,320$, 325,374 ; iii, 31 , I00, I32, I 53, I 86, I $90,289,297,305,308,314$, $324,338,345,367,375,383$, $389,394,4$ I 4,4 I 5,42 I, 432, 45I, 455 ; see also Wesleyans

Meyhec, Joan, iii, 76

Michael House (Cambs.), iii, 63

Michel, Michell, see Mitchell

Middeltone (Middletone Ernys), see Milton Ernest

Middle English, i, I 89

Middleho, man. (Hunts), i, 364, 365

Middlesex, Jas., earl of, iii, 276 ; Lionel, earl of, iii, 276

Middleton, see Milton Bryant and Milton Ernest

Middleton, Erasmus, iii, I 16 ; Mary, iii, 30I

Midland Association, ii, 44

Milbrook (Milbroke), see Millbrook

Mildentone, see Milton Ernest

Mildmay, Rob., iii, 236 ; Sir Walt., ii, 15 I

Miles, Rev. Hen. H., ii, 280 ; H., ii, 280

Militia, ii, 67

Mill (Mille), Cath., iii, I95 ; Jobn, iii, I95 ; Lewknor, iii, I95; Will. atte, i, 402 ; W., ii, 197

Mill-Bank, barrow (Dunstable), i, I 59

Millbrook, ii, 66, 84, 97, I I 5, I36, 324 ; iii, $252,267,275,308,3$ I $6-$ 320 ; adv., iii, 3 I9; char., iii, 3I9, ch., i, 3 I $5 n, 352$; iii, 3 I 8 ; crown property, iii, 27 ; man., iii, 3 I 7 , 326 ; mkts, and fairs, iii, 317 ; mills, iii, 316 ; place-names, iii, 317 ; sch., ii, 183 ; vicarage, i, $316 n$

Millbrook, Geoff., Ld., iii, 317 ; John, Ld., iii, 317

Millbrook (Milbrook), Walt., iii, I 47 Millbrook Warren (Millbrook), ii, I 46 ; $\mathrm{iii}, 317$

Mill Close (Goldington), iii, 206

Miller, Amicia, iii, I98; Maj. John, ii, 7o; John, ii, 358,363 ; J. B.,

ii, I 89 ; Will. the, iii, I98

Miller, Conquest \& Haynes, iii, 202

Miller's Bog (Pavenham), prehist. rem., iii, 76

Mill Farm (Eversholt), iii, 375

Mill Hill (Eversholt), iii, 375

Milligan, Dr., ii, $99 n$

Millo (Dunton), ii, 20I, 2 I 8, 219 ;

man., i, 312 ; ii, 220, 267

Millowbury (Dunton). ii, 2 I9
Millowbury Farm (Dunton), ii, 218

Millow Hall Farm (Dunton), ii, 2 I9 Mill Race, riv., iii, 399

Mills, Thos., iii, 293; J. T., iii, 404, 405,407 ; - iii, 401

Mills, ii, 74, I I 8, 2 I 2, 261, 262, 263, $266,268,269,275,276,278,283$, 297, 300, 301 $n, 302,310,320$, $323,324,336,34 I, 345,346,35 I$, $354 n, 355,356,357,358,360$, $366,376,381 n, 385$; iii, I I, 23 , $24,30 n, 37,38,42,44,47,51$, $59,60,64,65,66,69,73,74,78$, $82,85,89$, 9I, 96, I00, IO5, I I I I 4, I I 9, I 24, I 30, I32, I 33, I 36, I $38, I_{42}, I_{43}, I_{44}, 1_{47}, I_{49}$, I 50 , I 57 , I 7 I, I76, I 81,184 , I 86, I 89, 199, 202, 204, 206, $213,216,221$, $228,23 I, 235,244,245,247,254$, $256,259,260,271 \mathrm{n}, 275,284$, $286,287,289,297,302,303,307$, 3 I 4,3 I $6,325,326,340,35 \mathrm{I}, 370$, $372,375,379,380,386,387,389$, $394,395,400,408,409,418,453$ Mills Farm (Dunton), ii, 222

Milneho (hamlet), see Millo

Milnho, Rich. de, ii, 2 II ; iii, 260: Rog. de, ii, 211 ; iii, 260

Milreth, Beatrice, ii, 354 ; Will., ii,

Milton, Sir John, ii, 254; Thos.. iii, 60

Milton Bryant (Milton, Milton Bryan), i, 370 ; ii, 66, 106, I I 4 ; iii, $336,343,377,4^{1}$ 7-21 ; adv., iii, 42 I ; char., iii, 421 ; ch., $i$, 3 I 5 ; iii, 420 ; crown property, iii, 27I; Leys Farm, iii, 4I8; man., iii, 4I8, 419; Nonconf., iii, 4I8; parson, i, 330; placenames, iii, 4I8; schs., ii, I83: iii, 418,421

Milton Ernest, i, 324, $336 n, 384$; ii, I1 5,189 ; iii, $34,76,123$, I $25 n$, I 43-49, I $54 n, 183,224$; adv., iii, I 48 ; char., jii, I 44, I 49 ; ch., i, 3 I $5 n, 35$ I, 352 ; iii, I 47 . land rents, ii, I32; man,, iii, I 44 : Manor Farm, iii, I 44 ; mills, iii, I 30, I 44, I 47, 149; Nonconf., iii, I 44 ; Oakley Hunt kennels, iii, I 44 ; printing press, iii, I 44

Milton Ernest Hall, iii, I 44, I 49

Milton Grange (Milton Bryant), iii, 419

Milton Harnes, Milton Herneys, see Milton Ernest

Milton Hill, iii, I 43

Milton House (Castor, Northants), iii, 300

Milward (Mylward), Sir John, i, 33 I $n, 367 n, 368,402$; Rich., ii, 326 ; Will., ii, 334

Mineccan, Phil, iii, 220 n

Missenden Abbey (Bucks), i, 388 ; iii, 107

Mitchell (Micbell, Michel), Avery, iii, 195 ; John, iii, I95, 451 ; Rich. ii, 357 ; Rob., ii, 346 ; Thos., ii, 357 ; Will., iii, 50 ; W. C., ii, 190 Mitchener, Mrs., iii, 435

Moat Farm (Cranfield), iii, 275

Moat Farm (Marston Moretaine), iii, 308

Moat Hall (Eaton Bray), iii, 369

Moats, i, 303-8; ii, 226, 256, 288, $308,325,329,362 n, 376,378$, 384 ; iii, $2,8 n, 42,83,96$, I04, $109,124,136,153-4,157,160$, I 68 , I $7 \mathrm{I}, \mathrm{I} 8 \mathrm{I}, 186, \mathrm{I} 90,205,2 \mathrm{I} 2$, $233,242,275,276,284,289,297$, $305,308,31 \mathrm{I}, 314,320,324,329$, $369,370,375,379,390,400,42 \mathrm{I}$, $427,432,439,448,45 I$

Mobbs, E. R., ii, 200
Moddry (Clophill), iii, 316, 318; ch., i, 352 ; hermitage, i, $35 \mathrm{r}, 352$ Moding, iii, I07

Moggerhanger, i, 307, 380 ; ii, 21 , II 6, 209; iii, 228 ; adv., iii, 233 cb., iii, 228, 233 ; man., iii, 230 , 247; scb., ii, I83; woods, ii, 145

Moggerhanger House, iii, 228, 233

Moggerbanger Park, ii, I 47

Mohun, Christina, ii, 353 ; Isabel, ii, $306,35 \mathrm{I}, 352 n$; Joan, ii, 35I 353, 356, 36o; John de, ii, 306 , 351, 353; Mary de, ii, 306, 351 : Reg. de, ii, 306, 35I: Will. de, ii, $306,35 \mathrm{I}$

Mokehangre, see Moggerhanger

Mole, Anne, iii, $43^{8}$; Will., iii, $43^{8}$

Moleyns, Ld., iii, $436,43^{8}$

Molivers Wood (Bromham), iii, 44

Molluses, i, 69-7o

Molyneux, Ld., ii, 275

Monasteries, advs., i, 314; alien houses, i, 400,403 : dissolution of the, i, 330; feudal service, $i$ 319 ; grants to, $i, 316$; legacies to i, 325 ; parish chs. granted to i, 3I3, 3I5; property survey, ii 91 ; rights, i, 323

Monchesney (Monchensey), Beatrice ii, 203; iii, 13, 114; Will. de iii, I $3,46,52$, I I 4, I 59,383 : Sir Will. de, iii, 97

Money-pit (Totternhoe), earthwork, i, 294

Monkhouse, Rev. W., i, 297 ; iii, I 8,20

Monk's Close (Pulloxhill), ii, $376 n$

Monmouth, duke of, ii, 58 ; iii, $44 \mathrm{r}$; Chas., earl of, iii, I I

Monoux, Alice, iii, 334 ; Anne, iii 329 ; Eliz, Lady, iji, 423 ; Eliz. iii, 177, 329; Frances, ii, 243 Geo., iii, 329 ; Sir Humph., ii, 56 , I91, 243 ; iii, I $77,330,334,335$ Humph., ii, 41, 44; iii, 329, 331 : Lewis, iii, 329 ; Mary, iii, 330 , 335; Sir Phil., ii, $66 n, 69,242$, 243,245 ; iii, 20, 330, 334, 335 423 ; Thos., iii, 329 ; fam., ii, 66 ; iii, $276,331,334$

Montagu, John, marquess of, ii, 39 Montagu (Montague), Chas., see Manchester, duke of : Sir Edw. iii, 92 ; Edw., ii, 339 ; iii, I 55 I69; Geo., ii, 275 ; Geo. H., iii 387; Sir Hen., iii, 168; Mary, ii, 295; Rog., iii, I55; Sim., iii 155; Thos., ii, 295; iii, 126 ; Will., ii, 34 I ; iii, $3^{87}$; Ld. Chief Justice, ii, 96

Montaigne, Geo., bp., see Lincoln, bps. of

Montalt, John de, iii, $370 n$; Millicent de, iii, $370 n ; 448,449$

Montfort, Edm. de, iii, I 44: Hen de, ii, 30 ; iii, I 44 ; Sim. de, i $37^{2}, 373 n$; ii, $30,31,35$ I iii I44, 229, 353; see also Leicester, earl of : Thos, de, iii, I 44: Will. le, iii, I I $n$, I 22 ; fam., ii, 2 I $n$ Montgomery, Phil., earl of, jii, 290

Montgomery, Jane, iii, 39 I Lewis, iii, 39I

Monumental effigies, ii, 172, 2 I7 2 I $8,232,233,249,250,265,269$, $273,278,279,292,3$ I 2, 3I9, 324 $332,343,379$; iii, $28,39,48,68$ $76,120, I_{35}, I_{4} 8, I_{52}, I_{4}, 208$ $214,218,222,226,232,237,241$ $255,260,26 \mathrm{r}, 264-6,278,28 \mathrm{r}, 283$ $295,306,307,312,314-16,317 n$ $319,328,334,340,345,348,366$, $373,378,382,385,388,393,397$. 398,4 IO 4 I I , 4I3, 4I, 4 I 8,420 , $423,425,434,43^{8}, 44 I n, 445-6,46$ I 


\section{A HISTORY OF BEDFORDSHIRE}

Moody, Edm., iii, 340 ; F., iii, 340 ; Rob. S., iii, $34^{\circ}$; Ursula, iii, $34^{\circ}$ Moore (More), Edm., iii, 196 ; Eliz. i, $336 n$; Fran., ii, 146; iii, $3^{8} 4$; Sir Hen., i, $336 n$; iii, 198; Sir John, iii, 250 ; Dr. John, i, $336 n$; John, iii, 246, 415; Margery de la, ii, 276 ; Mary, iii, 246 ; Rich. iii, 201, 449; Rob. le (de la), ii, 25In, 276; Will., iii, 210; Dr. ii, 312

Moor End (Eaton Bray), cemetery, iii, 369; mill, iii, 370

Moor End (Felmersham), iii, 59

Moorland (Kempston), iii, 297

Moote, John, ii, 360 ; iii, 394

Moravians, i, 344 ; iii, 4, 9, 3I, $153,157,171$

Morcar, priest, i, 31 I ; ii, 356, 373 iii, 344,423

Mordaunt, bar, iii, III

Mordaunt, Lds., i, 331 n, 335 ; iii, $22,35,97,100, I_{I I} I_{14}, I 16$ 220 : Hen., ii, 40 ; iii, III, 326 327: John, iii, I 3, I $13,115,129 n$ 219, 329, 443; Lewis, iii, 5I, 96 I II, II 3, I I5, 253 ; John, vsct. ii, 56

Mordaunt, Agnes, iii, I10 $n$ : Alice, iii, IIo; Anne, iii, 13, I17, 221, 326: Cecilia, iii, 246, 247, 253 Chas., ij, 60 ; iii, III, I50; Edm. ii, 382 ; iii, I3, IIO, II 3, II 4 Eliz., iii, 326, 329 ; Ellen, iii, 110 Eustace, iii, 6o, II0, II2; Geo., iii, $246,247,253,254,259$; Hen., iii, Ir6, 253; Joan, iii, 97 Joane, Lady, iii, III: Sir John, iii, IIO, III, II6, 183 ; John, ii, 53 ; iii, 97, 98, I10, 23I, $247,253,332$ : see also Peterborough, earl of ; Lewis, ii, 39 iii, 332 ; Marg., iii, 326 ; Maud de iii, 324: Nichola de, iii, 324 Osmund, iii, 6o ; Rob., iii, 97, 98, 110, 112, 113: Will., ii, 147 iii, IIO, II $4, \mathrm{I}_{50}, 22 \mathrm{I}, 324$; W. wife of, i, $335 n$; fam., $i, 335$; ii, 56, 293 ; iii, 55, 109, 116

Mordaunts Manor (Turvey), iii, IIO ; mill., iii, II 4

More, fam., see Moore

More Close (Kempston), iii, 302

Morecote, John, iii, 380

Morewood, Frances, iii, 282 ; Gilb. iii, 282 ; Grace, iii, 282

Morgan, Anne, ii, 330 ; Ant., ii, 330 : Fran., ii, 330 ; Rob., ii, 278,324 , 343 ; Thos., ii, 330,378 ; Will., iii, I 95,398 ; iii, 326

Morice, see Morris

Morin (Moryn), John, i, 388 ; iii, 65 ; Philippa, iii, 67: Ralf, i, 388 , $389 n, 390 n ;$ iii, $65,66,67,212$; fam., iii, 212

Morins, Rich. de, i, 371, 372, $373 n$, $374,377,379,4$ OI $n$; iii, 363

Morinsbury, man. (Ravensden), iii, 212

Morker, John, iii, I 83, 216

Morkere, earl, $\mathrm{ii}, 22$

Morley, Agnes, iii, 106: Hawisia de, iii, 426 ; Nich., iii, 106; Rob. de, iii, 426 ; S. K., iii, I57

Morrell, Anne, iii, 407; John, iii, 407

Morrells, man. (Leighton Buzzard), iii, 407

Morris (Morice, Morres), Agnes, ii, 252 ; ii, 225; Alice, iii, 29r; Benj., ii, 359; Fran., iii, 255; Sir John, ii, 227; John, ii, 227, 252, 253, 329; iii, 225; Marg., ii, 329 ; Thos., iii, $129 n, 291$; Walt., iii, 143
Morrison, Sir Chas., iii, 253, 302, 376 ; Dorothy, Lady, iii, $376^{\circ}$ Dorothy, iii, 302 ; Eliz., iii, 254 : fam., iii, 324

Morsbury Hill, see Mossbury, earthwork

Morteyn, Constance de, iii, 309 : Edm. de, iii, 309, 433; Eliz. Lady, iii, 309 ; Eliz., i, 354, 357 ; iii, 23, 433: Eustace, iii, 433 Joan, iii, 406, 433; Sir John, iii, 309 ; John (de), iii, 309, 406, 433; Kath., iii, $386 n, 433$ : Thos., iii, 433 ; Will., iii, $386 n$, 433 ; fam., iii, 3II, 313; see also Morton

Morteynes man. (Stanbridge), iii, 407

Mortimer, Agatha, ii, 351, 352 ; Hen., ii, 352 ; Hugh, ii, 235, 306, $307,350,351,352,357,358,360$, 366 ; Isabel, $i, 391 n$; Joan, $i$, 352 ; John, iii, 405; Marg., ii, 235, 352 ; iii, 28I; Maud, ii, $352 n$; Petronilla de, ii, 307 , $352 n$; Rog., sec March, earl of ; Will. de, ii, 307, 352

Morton, Ann, iii, 368 ; John de, iii, 422 ; see also Morteyn

Mortuing, ii, 2 I 1

Morwen (Marwen, Morwenna), ii, 153; iii, 37

Moryn, see Morin

Mossbury, earthwork (Ravensden), i, 306,307 ; iii, 2 10, 212

Mossbury, man. (Tempsford), ii, $251,252,253$

Mosses (Musci), i, 56-8

Moths (Heterocera), i, 80-88

Motor-car industry, ii, I27

Moubray, see Mowbray

Moulsoe (Bucks), i, 357 ; iii, 399

Mounds, burial, see Burials and Tumuli

Mount Cabourn Camp (Sussex), Rom. rem., ii, 2, 6

Mounteagle, Lady, iji, I 92

Mount Hill (Flitwick), earthwork i, 286 ; iii, 284

Mountjoy, Ld., ii, 38

Mount Pleasant (Dunstable), prehist. rem., i, I60, I6I

Mount Pleasant (Kensworth), prebist. rem., i, I 66

Mowbray, Lds., iii, 30, 324 ; John, ii, 33; Thos., ii, 320 ; iii, 334

Mowbray (Moubray), Aliva de, ii, 300, 339; iii, 12, 262 ; Anne de, ii, 301, 340; iii, 13, 263; Eleanor de, ii, 30I ; Eliz. de, ii, 301, 339 iii, 263, 264; Isabel, ii, $34^{\circ}$; Sir John, i, 379 ; ii, 2 I I $n$; John de, i, $300,301,339$; iji, I2, 13, 44 I $37,262,263,264,329$; see also Norfolk, dk. of ; Maud de, ii, 300 , 341 ; iii, I2, 262, 329 ; Rog. de i, $300,339,344$; iii, $12,44,77$ 262, 329; Thos., i, 379; see also Norfolk, dk. of ; fam., ii, 223 , 235; iii, II, I $36 n, 205,215$ 433

Mowsbury Hill, see Mossbury, earthwork

Moxhill (Willington), iii, 238, 262

Moyne, Fulk le, iii, 248 ; Juliana le, ii, I95; Rob. le, ii, 248 ; Will. le, iii, 195

Mudge, Frances, ii, 379 ; Rob., ii, 379

Muggerhanger, see Moggerhanger

Muir, J. \& A., ii, I 20

Mulcaster, Rich., ii, 159

Mule, Nich. de, iii, 301

Multone, Thos. de, if, 28

Munchensy, see Monchesnev
Munt, - iii, 36 I

Munt \& Brown, iii, 35I

Mural paintings, see Wall paintings Murdak, Christine, i, 390 ; Hen., see York, archbps. of

Mursley, man. (Bucks), i, 370

Musgrave, Sir Chris., ii, 295 ; Edgar, ii, 296 ; Eliz. (de), ii, 295 ; iii, I62; Geo., ii, 296; Horace E., ii, 296; Joan de, iii, I62 ; Jos., ii, 295, 296 ; Rich. de, iii, I62; Rob. de, iii, I62

Mustard, Christine, i, 386 n ; Sim., iii, $38_{3}$

Muswell Hill, see Gooseberry-Bush Hill

Mycetozoa (Myxomycetes), i, 66-7

Myddleton, see Milton Bryant and Milton Ernest

Mydelton, see Milton Bryant and Milton Ernest

Mylton Harneys, see Milton Ernest

Mylward, see Milward

Myrnut, Walt. de, iii, 85

Nailour, Mary, iii, 235

Napier, Eliz., Lady, ii, 363 Frances, ii, 353; Sir John, ii, 295, 364 ; John, ij, 353 ; Penelope, ii, 373 ; Rich., ii, 307 ; Sir Rob., ii, $44,54,92,295,332,349 n$, $350,353,354,355,356,359,360$, $36 \mathrm{I}, 363,373,374,375$; Rob., ii, 307,356 ; Sir Theo., ii, $295 n$; Theo., ii, 353,375 ; fam., ii, I I9

Nares (Nares Gladley), man., see Gladley

Nares Gladley Farm, iii, 409

Neale (Neal, Neele), Bridg., iii, 415; Edm., ii, 360; Eliz., ii, 380 ; Esther, iii, 385 ; Hester, iii, I34; John, ii, 54 n; iii, I 34 , 135 ; Jos., ii, I $82 n$; iii, 1 36 , I65, I 70 ; Noah, iii, 157; Rich., iii, I34, I73; Thos., ij, 377,378 , 380 ; iii, I34, I 73 ; - , iii, I $32 n$

Needham, Bridg., ii, 338 ; Eustace, iii, 328 ; Fran., iii, I98, 294 ; Geo., iii, 328 ; Jer., iii, 328 ; Mary, iii, ı 98 ; Maurice, ïi, 328

Neele, see Neale

Negoose, Thos., ii, 156

Negus, Henry, iii, 103

Nene, riv., i, I 89 ; ii, 7 I

Neolithic Age, i, I 58-67, I74, 269 , $27^{\circ} ;$ ii, $34^{8}$

Netherbury, man. (Gt. Barford), see Burdelys

Nether Dean man. (Dean), iii, I 33 . 134

Nethersted (Colmworth), iii, I86 ; man. houses, i, 305

Nethertemple (Millbrook), iii, 318

Nevill (Neville), Alan de, ii, I 44; Anne, iii, I4 ; Dorothy, iii, I4, 235,330 ; Eli2., ii, 247, 275 ; ii, 13 ; Geo., iii, I 3 ; see also Bedford, duke of : Hen., iii, I 4 ; Sir John, iii, 21 5, 235, 330; John (de), ii, $203,247,275$; iii, I3, I4 ; Kath., iii, 14 ; Lucy, iii, 14,46 ; Nich. de, ii, 289 ; iii, $63 n$; Sir Rich., iii, 46 ; Rich., iii, 14 ; fam., iii, $46,77,96,181,212,331,383$

New (Luton), ii, 361

Newark, Ad. of, i, 353

Newbottle, man., i, 376,377

Newbury (Newberry) man. (Silsoe), ii, $329,330,332$

Newbury Farm Moat (Silsoe), ii, 325

Newby, Tbos. de, iii, 276

Newcastle, dk. of, ii, $62 n$; Will., dk. of, iii, 43 ; earl of, ii, 345 


\section{INDEX}

New College (Oxford), ii, I57, I60-75; iii, 324, 425

Newcombe, Steph., iii, r 22

Tewcome, -, bellfounders, ii, 226, $245,254,299,312$; iii, 29, 63, $94,148,208,233,250,278,283$, $323,393,398$

Newdigate, Anne, ii, 322 ; Ant., ii, 343 : Sir John, ii, r9o; John, iii, 326 ; Sir Rich., iii, 289 ; Sir Rob., ii, 339, 340 ; Rob., ii, 340, 34I ; iii, 326; Thos., ii, 322,323

Newebond, Gunnora, de, ii, 255 ; Nich. de, ii, 255

Newell (Newill), Gabriel, iii, 280; John, iii, 280; Lucretia, iii, 80

Newenham, Ann, iii, 72 ; Will., iii, 72

Newes, Pet., iii, 259

Newhouse, abbot of, i, 314, 353

Newill, see Newell

Newman, Anne, ii, 235; Barth., iii, 225: Cecilia, iii, 225; Dan., ii, 235; Eliz., ii, $235 n$; John, iii, 184; Thos., ii, 24I ; iii, 200

Newmarket, ii, I 91

Newmarket Heath, earthworks, i, I 76

New Mill End (Luton), ii, 374

Newnham (Goldington), i, $315 n$, $316 n, 362 n, 379$; iii, 202 ; A.S. rem., i, I86; man., iii, 205

Newnham, man. (Biddenham), iii, 37

Newnham Priory, i, $303,305,313$, $314,315 n, 317,322,326,33$ I $n$, $332,349,350,354,362,37 \mathrm{I} n$, $372 n, 377-85$; ii, $145,153,154$, $215,257,258,301,338$; iii, 2 , II, I2, I6 n, 24, 29, 37, 88, $90,91,95,97,98,100, \mathrm{II}_{4}, \mathrm{r} 80$, 202-6, 208, 210, 212, 214, 216, $22 \mathrm{I}, 235,236,237,258,26 \mathrm{I}, 264$ $266,331,334,335,342,353,378$, $406,424,425$; endowment, i, 350 , 380 ; ii, 218 ; foundation, i, 377, 39 r $n$; mills, i, 305 ; ii, I55; iii, 206; priors, i, 328, $372 n, 38 r$, 391 ; ii, I54, I55, $218 n$; iii, I 2, $23,30,90,184,185,203,204,216$, 236, 342

Newport, Anne, ctss. of, ii, 347,382 ; Mountjoy, earl of, ii, 347,382

Newport, Mercia, ii, $378 n$; Will., ii, $37^{8 n}$

Newport Pagnell (Bucks), ii, 46, $47,48,49,5 \mathrm{I}$; iii, $27 \mathrm{I}$

New Rowney Farm (Southill), iii, 259

Newton (Dunton), ii, 218, 2 19

Newton, Frances, ii, 378 ; Humph., iii, 2 I $n$; John, iii, 2 I $n$; Rich. de, i, 40r, 402 ; Will., ii, 378 ; iii, $2 \mathrm{I} n ;-, \mathrm{iii}, 3 \mathrm{I}$

Newton Blossomville (Bucks), iii, II 4

Newton Bromswold (Northants), ii, 71 ; iii, I23, I75

Newtonbury, man. (Dunton), see

Dunton Chamberlain

Nichol, see Nicoll

Nicholas, archd. of Beds, i, 313 , $379 n, 390 n$

Nicholas's Mill (Flitwick), iii, 287

Nicoll (Nichol), Edw., iii, 429; Thos., iii, 429; Will,, iii, 3 r

Nicolls, Mary, iii, 388 ; Rob., iii, 27 ; Rog., iii, 73

Noble, Geo., ii, 270

Nodes, Chas., ii, 234 ; Geo., ii, 234 286,287 ; iii, 39 ; Helen, iii, 39 . John, ii, 382 ; iii, 261

Noel, Jas., ii, 235 ; John, ii, 235

No-man's-land Common (Herts), palæolithic impl., i, I 50
Nones Stocking Close (Chicksands), ii, 266

Norbury (Norbery), Anne, iii, 78 , 373 ; Sir Hen., iii, 78 ; Joan, iii, 78 ; Sir John, iii, 78 ; Will., iii, r26

Norfolk, assessment of, ii, $4^{8} n$

Norfolk, ctises. of, Constance, iii,

12: Marg., i, 35I $n$ : Maud, iii, 439; dchsses. of, Eliz., ii, 223 , 267 , 301, 340; Eleanor, ii, 268, 275 ; dks. of, i, 363,364 ; iii, I $66 \mathrm{n}, 2 \mathrm{I} \mathrm{I}, 237,238,239$; iii, 4 I8; John, ii, $220 n, 223,268$, $275,283 n$, 3or ; Phil., iii, r92; Thos., ii, $267 n$, 30I, $34^{\circ}$; iii, I2, 4 I, III, 263 ; earls, ii, 220, 223, 268; iii, 7 I $n$; John, iii, I2 ; Rog., iii, 439 ; Thos., ii, 339 ; iii, 12

Norgewele, see Northill

Norman, iii, 247

Norman, Rob., ii, $293 n$

Norrell, see Northill

Norris, Hen., iii, I72 ; Sir John, ii, 46 ; Sarah E., iii, I87 ; Toby, ii, 299 ; iii, 135

Nortgible, see Northill

Nortgivele, see Northill

Nortgylle, see Northill

North, Chas., iii, I 5 I ; Sir Edw., iii, 30 ; Gilb., iii, 123,180

Northampton, iii, 1o; Hosp. of Holy Trinity, iii, 40

Northampton, earldom of, iii, 408

Northampton, earls of, iii, 209, 428 ; Sim., i, $3^{87} n, 3^{88}$; ii, 255 ; Will., iii, 168, 169, 172, 208

Northampton, Rich. of, i, 353

Northamptonshire, lace ind., ii, $122, \mathrm{r} 23, \mathrm{r} 24$

Northaw Wood, ii, $361 n$

North End (Stagsden), iii, 96

North End Farm (Odell), iii, 69

Northewell, John de, ii, 362

Northfleet, Thos. de, ii, 214

Northiell, see Northill

Northill, i, 33r, 365, 403; ii, 6, 43, $95 n$, I16; iii, 175, 176, 227, 238, 239, 242-5r ; adv., iii, 250 ; anct. burials, ii, I ; chant., i, 329; ii, 92 ; char., iii, 25r ; chs., i, 33I, 349,403 ; iii, 249,273 ; copyhold at, ii, 9o ; mans,, ii, 77 ; iii, 243 ; mills, iii, 247 ; rectors, i, 403 ; Rom. rem., ii, 9 ; iii, 243 ; schs., ii, 183 ; iii, 242

Northill College, i, 403; ii, 92, 25I ; iii, 244, 250

Northill College Farm, iii, 244

Northill College Manor, iii, 244

Northumberland, earldom of, ii, 20,22

Northumberland, Katb., ctss. of, iii, I 4 ; earls of, ii, 42 ; Hen., iii, 14 ; Siward, iii, 168; Tostig, ii, 22 : Uhtred, ii, 20

Northwood, man. (Luton), see Norwood'

Northwood (Northwode), Alice de, ii, 328 ; Gilb. de, iii, $396 n$; Hen. de, ii, 328 ; iii, $320,323,396$; John de, iii, 323,396 ; Rich. de, ii, 328 ; Sim. de, i, 327 ; Thos. de, ii, 328

Northwood End (Haynes), ii, $33^{8}$

Norton, Alice de, iii, 224 ; Brett, iii, 259; Eliz. de, ii, 322; Sir -Geo., ii, 363 ; Graveley, ii, 382 ; Hen., ii, $3^{82}$; Isabella (de), ii, $3^{8} 3$; Sir John, ii, 377, 380; John, ii, 312 ; Lettice, ii, $382 n$; Luke, ii, $361,382,383$; Pet. de, ii, 322 ; Sir Ralph de, ii, 322 ; Rich., ii, I $84 n, 3$ ro, 312, 360, 377, $3^{83}$,
Norton (cont.)

384 ; Rob., ii, 382 ; Sarah, iii, 259; Thos. (de), ii, 322, 376, $377,3^{82}, 3^{8} 3$; Walt., ii, 382,383 Will. (de), ii, 382, 383; iii, 224 ; Col., ii, 47

Nortyevele, see Northil

Norwell, see Northill

Norwich, Dr. Tanners, archd. of ii, 17o ; Fran. White, bp. of, ii, 162

Norwich, Mary, ctss, of, iii, 330 ; Edw., earl of, iii, 330

Norwich, Anne, iii, 340; Greg., i, 387 ; Ralph, iii, $34^{\circ}$

Norwood (Ridgmont), iii, 323

Norwood, man. (Luton), ii, 350,363

Norwood, man. (Silsoe), ii, 328, 329,332 ; chant., ii, 333

Norwood, Edm., iii, 326 ; John, iii, 326 ; Thos., iii, 326

Notley Grange (Bucks), ii, $3^{8} 5$

Notley Priory (Bucks), i, $3^{8} 7 n$, $388 n ; \mathrm{ii}, 273,385$

Nottinge, see Knotting

Nottingham, Anne, ctss. of, ii, 340 John, earl of, ii, 339 ; iii, I2, 263 ; Thos., earl of, see Norfolk, dk. of Will., ear] of, ii, 301,340

Nowers, Grace de, ii, $35^{8}$; Joan, iii, II 8 ; John de, ii, 358 ; iii, II 8 Rich. de, iii, II8; Rog. de, iii, I 8 , II9, 122

Nunwood (Harrold), iii, 64, 66

Nurse, Will., ii, $342 n$

Nussa, Terricus de, i, 39

Nutley (Bucks), see Notley (Bucks)

Nutley, Luke of, i, 40I, $4^{\circ 2}$

Nyandser, John, ii, $3^{8} 5 n$; Marg. ii, $3^{8} 5 n$

Oakden, Sim., iii, r6 65

Oakely, see Oakley

Oakes Farm (Kempston), iii, 302

Oaklands (Aspley Guise), iii, $33^{8}$

Oakley, i, $335 n$; ii, I1 $5,166,188$; iii, $76,123,128,130,131$ I 49-53; adv., i, $3^{84}$; iii, I 52 bridge, iii, I 49, I50; chant., iii, I53 ; char, iii, I 53 ; ch., i, 3 I $5 n$, 318,382 ; iii, I3o, I 3 r, I 32 , I 5 I fishery, iii, I49; mans., iii, I 49 : mills, iii, 149, I50; Nonconf., iii, I 53 ; place-names, iii, I 49; sch. ii, 183 ; vicarage, i, 317 ; witchcraft at, ii, Ioo; iii, I 49

Oakley, Fran., iii, 9; Will., iii, I $8 n$

Oakley Club, ii, I89

Oakley Hill, iii, 149

Oakley House (Oakley), ii, 187 ; iii, I 49

Oakley Hunt, ii, I87-90 ; iii, I44

Oakley Hunt steeplechases, ii, 194

Oakley Reynes, man. (Oakley), iii, I 49

Obness (Bolnhurst), i, 276

Occliffe, see Hockliffe

Ocle, see Oakley

Ocle-cum-Clapham, man. (Clapham), iii, I 30 , I 50

Octona, see Houghton Conquest

Odecroft, hun., iii, 39 I

Odell, ii, 44, II 5 ; iii, $34,50,54,59$, $69-76,77,100,102$; adv., iii, 76 ; ch., i, 300; iii, 75 ; earthwork i, 300 ; fairs and mkts., ii, 88 iii, $35,69,73$; forest offences ii, I44; ind., iii, 69 ; land values, ii, 92 ; mans., iii, $73-74$; mills, i, 300; iii, $59,73,74$; sch., ii, 184 Odell, bar., i, 300 ; ii, $30 n, 234 n$ $282,326,328$; iii, $69,71-4,84$ 92, I05, I 06, I I3, I 8, I 46,3 I 4 $32 \mathrm{I}, 322,44^{8}, 449$ 


\section{A HISTORY OF BEDFORDSHIRE}

Odell, hon. of, ii, 283 ; iii, 82, 159

Odell, Little, man., iii, $69,73,74$

Odell, Rob. (of), i, 365; iii, 46, 3 II ;

Steph., iii, 3 I ; Thos., iii, 31 I ; see also Wahull

Odell Castle, i, 300 ; iii, 69, 73, 102

Odell woods, iii, 69

Odley, Odyll, see Odell

Offa, king of Mercia, i, 190, 309, 3 Io, 3I5 $n$; ii, 20, 3I 4,36 ; iii, I5I, 401

Offley (Herts), iii, 349

Offley, John, ii, 63; Sir Thos., ii, I6r:-

Oger, ii, 22

Oilgi, Oilli, de, see Doyley

Okele, see Oakley

Okey, Thos., iii, 323 ; Col., ii, 55 ; iii, $64,271,404$

Okley, see Oakley

Olaf, priest, ii, 34

Oldfeild, Rich., ii, 229 ; Rob., ii, 299, 373

Oldham, Hugh, i, 399 ; iii, 408; Sim., iii, 201

Old Ramerick Farm (Holwell), ii, 286

Old Rowney Farm (Southill), iii, 259

Olive Ground, close (Leighton Buzzard), iii, 404

Oliver, Jas., iii, I 54 ; Jane, iii, 203 . John, ii, $285 n$; N. D. T., ii, I99 : Sim., iii, I54: Will., iii, 203 Oliver's Ditch (Clapham), iii, 130

Olivier, Dan. S., ii, $182 n, 280$ Mary A., ii, 280 ; Susanna, ii, 280 Olney (Bucks), ii, $187 ;$ iii, 418 mkt., iii, 69

Olney, Goditha, iii, 219 ; Rob., iii, 219

Ombersley, barons of, ii, 330

O'Neill, Rev. - ii, 374

Ongley, Lds., ii, 7o, 231 ; iii, 253 254 ; Rob., iii, 253, 257

Ongley, E., ii, I94; Frances, ii, 243 F., ii, r94; R. H., ii, 63, 64 ; Sir Sam., iii, 255 ; Sam., ii, 6o, 62,243

Ordui (Ordwig), iii, 38, 258, 327

Ordway, John, ii, 385

Ordwig, see Ordui

Orewell, Alan de, iii, 21

Orford, Edw., earl of, iii, 376

Orkneys, the, straw-hat ind., ii, 120

Orlebar, Rev, A., i, 283; Diana iii, 6o, 81, 87 ; Geo., iii, 82,86 , 233: Jane, iii, 86 ; John, iii, 82 $85 n, 94,146$; Judith, iii, 60 ; Marg., iii, 86, 233 ; Rich., ii, 59, 69 ; iii, 81, 82, 83, 86, 87 ; Rich. R. B., iii, $81,82,83,87$; Thos., iii, 6o, 87; Ursula, iii, 6o; Will. A., iii, 84, 87; Mrs., ii, I22 ; -, iii, 80

Ormond, dk. of, iii, 4

Ormond, Joan, iii, Igr ; John, iii, I9I

Ormsby, Arth., iii, I 54 ; Marg., iij, 154

Ornaments, A.S., i, I79, $18 \mathrm{r}$, I82, $184,187,189$, I90; ii, 266 ; iii, 57, 402 : Rom., ii, 6, IO, I4, I5 ; Rom.-Brit., ii, II, I4

Orwelle Meade (Clifton), ii, 278

Osbaldiston, Dorothy, iii, 236 ; John, iii, 236 ; Rich., iii, 236

Osbern, iii, 42, 52, 92, 195, $207 x$

Osbert, i, 313; iii, I1 8, I38, 448

Osborn (Osborne), Sir Algernon, ii 197 ; Sir Algernon K. B., ii, 270 , 271, 273, 291, 342, 344; Alice, iii, 39; Sir Daniel, ii, 270 ; Sir Danvers, ii, 6r, $66 n, 273$; Dorothy, ii, 275 ; Sir Edw., iii, 244: Edw., iii, 39, 244, 250 :
Osborn (cont.)

Eliz., iii, 244 ; Sir Geo., ii, $63 n$, $64,69,268,273,29 \mathrm{I}$; Sir Geo. R., ii, $66 n$ : Hen., ii, 271 ; Sir John, ii, 69, 269, 270, 271, 273, 275, 342 : John, ii, $59,66 n, 276$; Sir Pet., ii, 43, 27I, 273, 344 ; Pet., ii, 271, 275, 276, 342, 344 : Sarah, ii, 6I, 280 ; Thos., ii, 270 , 280 ; Mrs., ii, 62, $63 n, 64$; iii, 428

Usborne, Ld. F. G., ii, I93

Oseney Abbey, see Osney

Osgodby, Ad. de, iii, 46r

Osiet, iii, $46,24^{8}$

Oskytel, archbp., see York, archbps. of

Osmund, canon, iii, 37

Osney Abbey, i, 383 ; iii, I $53 n$

Ossington (Notts), i, 394

Ossory, earl of, ii, 192

Ossory, Upper, earl of, see Upper Ossory

Osulf, iii, II 2, 149

Oswulf, iii, 431

Ottobon, ii, 32

Ottway, Rich., iii, 63

Oughtred, Sir Ant., iii, $15^{8}$

Oundle, ii, I $7 n$

Ouse, man. (Sharnbrook), iii, 91, 92

Ouse, riv., i, I, 27, 28, 43; ii, 7I earthworks near, i, 280 ; isls. in, iii, 195, 202 ; Rom.-Brit. settlement near, ii, 5

Ousel (Ouzel or Lovat), riv, ii, 71

Oustone, see Houghton Conquest

Over (Cambs.), ii, $24 \mathrm{I}$

Overdean, man. (Dean), iii, 133-4 ; mills, iil, 133

Overhey Wood, iii, 43

Overstone (Northants), iii, 122

Ovy, Anne, iii, 97 ; Nich., iii, 97 ; Philippa, iii, 97

Ow, Ailmar d', iii, 258; Will. de, ii, $267 n$

Owen, Jane, iii, 265 ; Hen., iii, 265 ; fam., iii, 266

Owens \& Co., ii, 26r

Ox Close (Goldington), iii, $208 n$

Oxenbridge, Frideswide, Lady, iii,

343 : Sir Thos., iii, 343

Oxenbrigge, John, ii, 299

Oxford, iii, I6, I7 ; siege, ii, 49

Oxford, Isabel, ctss. of, i, $366 n$; iii, 314 ; earls of, iii, 310 ; Hugh, iii, 314; John, iii, 263; Rob., iii, $3 I_{4}, 459 n$

Oxfordshire, lace ind., ii, I23

Oxhey Chapel, ii, 317

Oyldeboef (Wildeboef), John, i, 386 ; iii, 239, 332 ; Laura, iii, 239, 332 ; Marg. de, iii, 187; Ralf de, iii, I86 : Rich. de, iii, I86; Walt., iii, 186 , 187 ; Will., iii, I54, I 86 Oyley, d', see Doyley

Ozingell, i, I8I $n$

Pabenham, par., see Pavenham

Pabenham (Pavenham), Agnes, iii 4I: Alice, iii, 50; Athelina, iii, 5o; Eleanor, iii, 77; Elena de. iii, 223 ; Eliz., ii, 243 ; iii, 57, 77 Sir Jas., iii, $21 \mathrm{I}$; Jas. de, iii, 50 , $224,259,330$ : Joan, iii, 77,259 Sir John de, iii, 258 ; John de, ii, 145 ; iii, $4 \mathrm{I}, 43,50,57,64,67$ $77,79,83,85,223,224,226,258$. Kath., iii, 77 , r91 ; Laur. de, ii, 243 : iii, 64, 77, 191; Margery, iii, 50, $211,224,330$; Rich. de, iii, 67: Thos., iii, 77, 85, 224

Pack \& Chapman, ii, 373 ; iii, 366

Page, Chas., iii, 416; Eliz., Lady,

iii, 435; Eliz., iii, 435; Frances,
Page (cont.)

ii, 379 ; Sir Greg., iii, 344 ; Hen. iii, 76 ; Jobn, ii, 379, 380 : Matth. ii, 377 ; Pet., ii, 377 ; iii, 375; Sir Rich., iii, 385, 435 ; Rich., ii, 379. 380 ; Will. S., iii, 4 I6

Paget (Pagett), Sir Will., ii, 377 ; iii, 443 ; Rev., -, iii, I 54

Page-Turner, Sir Edw., ii, 363, 385 : F. A., ii, $363,383,385$, 387 ; iii, 434, 435; Sir Greg., ii, 385 ; iii, $343,344,422,434$; fam., iii, 435

Pakenham Park (Carlton), iii, 50

Pakinton, i. 360

Palace Yard (Splashyard), wood, (Roxton), i, 302

Palæolithic implements, see Implements

Palæolithic races, i, I5

Palæolithic rem., i, I $45-58$

Palæontology, i, 33-35

Palldocke, John, iii, 208

Palmer, Chas, iii, 236, 253: Chas. F., ii, I 89 ; iii, 245 ; Dorothy, iii, 236; Rev. Fran., ii, 246 ; Fran., iii, I $4 n$; G., ii, 48 ; Hen., ii, 277 ; Hen. F. iii, 245; John S., ii, 358 Madeline, Lady, iii, 245: Rob., iii, 253; Sam., iii, 9 ; Sir Thcs., ii, 268, $358 n, 377,380$; Thos, ii, 329 ; iii, 253 ; Sir Will., ii, 43 ; iii, 253 ; Will., iii, I62; -, iii, 52

Pannells Grove (Leighton Buzzard), iii, 409

Papiatem (Caddington), ii, 3 I 5

Papley, Walt., iii, 312

Paradine, Capell, iii, 25 ; John, iii, 25; Martha, iii, 25; Mary, iii, 31 ; Rob., iii, I56, 226; Sarah, iii, 33 : Thos., iii, 22, 32 ; see also Parentine

Parage, Rob., i, $350 n$

Parcell (Parsell), Mary, iii, 247 ;

Nath., iii, 247 ; Will., ii, 29I

Pardoe, Rob., iii, 430

Pare, John, iii, 392

Parentine (Parentin), Ellen de, iii, 9I ; Rob., iii, 74, 90, 91 ; Will., iii, I59; fam., iii, 91 ; see also Paradine

Parentines, man. (Sharnbrook), iii, 9 I

Pares, Thos., ii, 296

Paris (Parys), Amicia, ii, 330 : Fulk of, ii, 2II; Matth., i, 3 ro 354, 359; Rob. de, i, 396, 397 ; iii, 330 ; Will. de, i, 352

Parke (Park), Sir Jas., iii, 270 ; Rog., ii, 34I

Park End (Stevington), iij, Ioo

Parker, Hen., iii, 349; John, iii, $325 ;-, i, 345$

Park Farm (Eaton Bray), iii, 369

Park Grange (Old Warden), iii, 254 Park House Farm (Podington), iii, 80

Parkinson, W., iii, 428

Parks, ii, 146-7

Park Wood (Harrold), iii, 64

Parliamentary representation, ii, 33-4

Parr, Eliz., iii, 72 ; Hen., iii, 392 Kath., iii, 14 ; Sir Will., iii, 72 ; -, ii, 194

Parratt, Thos., ii, 40

Parry, - , ii, I2 I

Parsonage Close (Leighton Buzzard), iji, 415, 416

Parsonage Farm (Haynes), ii, $342 n$

Parsons, John, iii, 301 ; Kath., iii, 301; Thos., iii, 301 ; Will., iii, $332:-, \mathrm{ii}, \mathrm{I} 65$ 


\section{INDEX}

Partington, Thos., ii, 299

Partridge, Jos. A., iii, 259, 276 ; Steph., iii, I5I; Wharton, iii, I28; Mrs., ii, $3 I_{4}$

Parys, see Paris

Paschall, Rob., iii, 45I

Passelowe (Passelewe, Passaquam), Alice de, ii, 283; Amicabilia, iii, $36 n$; Gilb., iii, $36,37,44 n$, $386,387 n$; Joan, iii, 46 ; Ralph, iii, $36,39,343,345,386$; Rob., ii, 283,382 ; iii, 339 ; Thos., iii, 36; Will (de), ii, 283 ; iii, 36, 38, 39,46

Paston, Agnes, iii, 60, 107, 158, I 87

Patenham, see Pavenham

Patersen, John W., iii, 447

Patishull (Pateshull, Pattishall), Agnes de, iji, 4I ; Alice (de), iii, 45 ; iii, 136 ; Isabel, iii, 45 ; Sir John de, i, $325 n$; John de, ii, $34 n$; iii, I5, 4I, 42, 45, 46, 52 , $61,71 n, 105,107,137,215,329$, 330 ; Kath. de, iii, I5, 1 36, 330 ; Mabel, Lady de, i, 395 ; Mabel de, iii, 330; Martin, ii, 28; Sibyl de, iii, 4I, I36; Sir Sim. de, iii, 45; Sim. de, i, 366, 385; ii, 30 ; iii, I4, 40,6I, 107, 184,330; Walt. de, iii, $4^{\circ}$; Will. (de), iii, I5, 4I, 45, 1 36, 21 5, 330, 424 ; -, Lady, i, $355 n$; fam., iii, $91, x 81,206$, 235

Patnam, see Pavenham

Patten, Mary A., iii, 274

Pattishall (Northants), i, 37 I $n$,

376,377
Paulet (Powlett), Agnes, Lady, iii, 384; Anne, iii, I77; Eliz., iii, I77; Sir Will., iii, 277,384 ; Will., iii, I 77

Pauncefote, Isabel, i, $386 n$

Paunfort Lodge (Ampthill), iii, 268

Paveley, Joan, iii, I43; Rich., iii, $\mathrm{I}_{43}$

Pavement, Rom., ii, 12, 223

Pavenham, i, 318; ii, I1 5, I24, I 39 ; iii, $34,59,60,76-80,100, \mathrm{IO}_{4}$, I 43 ; adv., iii, 80 ; char., iii, 80 ; ch., i, 318; iii, 63, 79 ; inclosure, iii, 80 , inds., ii, $124-5$; iii, 76 ; mans., iii, 49, 50, $5 \mathrm{I} n, 57$, $76-79,83,330$; mill, iii, 78 ; sch., ii, I 84

Pavenham, bar., iii, $248 n$

Pavenham, fam., see Pabenliam

Pavenham Bury (Pavenham), iii, 76,145

Pavenham rush matting and wicker basket industries, ii, 124-5

Paxton, Gt. (Hunts), ii, 246

Paxton, Edm., iii, 18 ; Sir Jos., iii, 343

Payne (Payn), Cath., ii, 242 ; Sir Chas., ii, 252 ; Chas., iii, $33^{\circ}$; Chas. S., iii, 228 ; Sir C. G., ii, $66 n$; Dan., iii, 85,87 ; Fran., iii,, 82, I19; Sir Gillies (Gillias), ii, 65,252 ; iii, 330,335 ; John, iii, 330 ; Mary, iii, 330,335 ; Sir Pet., ii, $66 n$; Pet., iii, $330 n$; Phil., iii, 330 ; Rob. le, iii, $186 n$; Sybil, iii, 82, II9; Thos., iii, 60, 82 ; Will., iii, $82,85,86,87$, II9; 一, ii, 65

Paynel (Paynell), Eliz., ii, 345 ; Joan, iii, 234, 33I ; Ralph, ii $345 n$; iii, $45,46,52,98,234$ ' $33 \mathrm{r}$; Thos., ii, 330,345 ; fam.' iii, 206

Peake, Edw., ii, I 79, 21 5, 255

Pearce, Mary, ii, 317

Pearles, Anne, iii, I19

Pearse, -, iii, 277
Pearson, Geo. J. H., ii, 246 ; see also Pierson

Pear Tree Close (Turvey), iii, i I 4 n

Peasants' Rising, ii, 35, 90

Peat, Thos., ii, $185 n$; iii, 226

Peche, Hugh, iii, 229 ; Ida, iii, 229

Pechesdone, see Pegsdon

Peck, John, ii, 363 ; iii, 183 ; R., iii, 218 ; Will, ii, 36

Peckham, archbp., i, 375

Pecohe, Edw., iii, I 56

Peddar (Pedder), Agnes, iii, 366 ; Alys, iii, 425; John, iii, 366, 425 ; Marg., iii, 366 ; Matilda, iii, 366 ; fam., iii, 424

Pedley, John, ii, 3r8; Will., iii, I83; W., iii, I85; 一, ii, I33: iii, 183 ; fam., ii, 316 ; iii, 184

Peel, vsct., ii, 66, 23I, 242, 244

Peel, Arth., ii, 23I ; B. L., ii, 199 Sir Rob., ii, 244 ; Sir Will., ii,

Peers, John, iii, 3

Pegsdon (Shillington), ii, 25, 79 294, $295 n, 296,299$; farm, ii, 82 ; man., i, 312 ; ii, 296, 297

Peile, Dr., ii, 374

Pekesdene, see Pegsdon

Pelham, Chas. A., see Yarborough

Peling, man., see Pillage, man.

Pemberton, Goddard, ii, 54, 55 John, iii, 208, 311, 333; Sir Lewis, ii, 268: Mary, ii, 268 Ralph, iii, 333 ; Rob., iii, 333 ; Rog., iii, 333

Pembridge, Sir Fulk, iii, 299 Marg., Lady, iii, 299

Pembroke, ctsses. of, iii, 40 ; Eleanor, ii, 306,350 ; Isabel, ii, 384 Joan, ii, 223; Mary, ii, 290 Maud, ii, $223 n$; earls of, ii, 211 224,227 ; iii, 64, 314; Anselm Marshal, ii, 223, 351 ; Aymer de Valence, ii, 275; Gilb., ii, 226 , 228, 229; John Hastings, ii, 238 ; Laur. de Hastings, iii, 229 ; Walt. Marshal, ii, 220, 267 Will. Herbert, iii, I 73; Will Marshal, ii, $306,350,35^{6}, 3^{84}$ 439, 44I, 442; Will. de Valence ii, 223

Pembroke Hall (Camb.), iii, 248

Pendrid, Will, ii, 245

Penne, John de la, iii, I 33

Penneclose (Wootton), iii, 333

Pennefather, Edw., ii, 380

Pennyfathers Hill (Maulden), iii, 313

Penruddocke, Sir Thos., iii, 196

Penthelyn, John, ii, 373

Penyston, Fran., iii, 442, 454

Penythorn, Will., iii, I 45

Perche, count of, ii, $350 n$; Geoff., iii, 439, 442, $44^{6}$

Percival, Sir John, ii, 158,159

Perdington, Maud, iii, 2 I I

Perot, see Pirot

Perots (Beeston-Caldecote, Trumpingtons), man. (Northill), $\mathrm{iri}, 24^{8}$

Perry Hill Farm (Cranfield), iii, 278

Personal Strongholds, i, 285-303

Pertenhall (Pertenhale), i, $297 n$; ii, 72 , I1 5 ; iii, $123,137,138$, I53-7, I59 ; adv., iij, I56 ; char., ii, $184 n$; iii, 157 ; ch., $i, 337 n$; iii, 155 ; man., iii, $153,154,166$; moats, iii, 153 ; Nonconf., iii, I 53 : sch., ii, 184

Pertenhall, Eustace de, iii, I55n; Rich. de, iii, 155

Pertenhall Hoo Farm, iii, ${ }^{1} 53$

Pertesoil, Athelina, iii, I5?; Eliz., iii, I59; John, iii, I59; Rob., iii, I 59 : Rog., iii, I59; Sim., iii, I 59 ; Will., iii, I 59
Pertsoills (Pertesoyles), man. (Risley), iii, I 59

Perys, Agnes, ii, zog; Thos., ii, 209

Peshall (Peshale), Aliva, ii, 300 , 339 ; Sir Rich., ii, 339; iii, 263

Peterborough, R. Clavering, bp. of, iii, 179

Peterborough, earls of, ii, $40 n, 53$, 56, 59, 60 ; iii, II 4-1 7 ; Chas. H., iii, III ; Hen., ii, 43 ; iii, 5 I, I II ; John, ii, 293; iii, 5I, III; fam., iii, $37 n$

Peterborough Abbey, i, 312 ; iii, 70 Peters, Rich., ii, 357

Petever, Alice, iii, 355 ; John, iii, 355

etre, Eliz, iii, 203, 209: Sir John, iii, 203 ; John, iii, 209 ; Sir Will., iii, $203,218 n$; Dr., i, 367,368

Pettit, fam., iii, 388

Pever (Peyver, Peyvre), Avice, iii, $440 n$; Beatrice, iii, 442 ; John, ii, 328 ; iii, $153,440-2,446 n$. Marg., i, 402 ; iii, 346, 366, 440 : Maria, iii, 442; Mary, iii, 346 $366,440,44^{2}$; Nich., iii, 137 , 153, 440, 442; Paul (Paulinus), i, $325 n$; iii, $343,439,440,444$ 446 ; Rog., iii, I53, 346, 444 Thos., i, 402 ; iii, $346,366,440-2$, 453 ; fam., iii, I66, 446

Peverel, hon. of, iii, 57, 223, 225, 433

Peverel (Peverell, Peveril), Edm. ii, 224, 227, 263; Isabella, ii, $22+n$; John, ii, 224, 227 Jordan, iii, 372 ; Marg., ii, 224 Rob., ii, 309, 3 I0; Will., i, 314 ; iii, $57,86,433$; fam. of, ii, 262

Peyncourt, Eglina, iii, 59; Nich. iii, 59 ; Rich., iii, 59 ; Walt., iii, 60

Peyton (Payton), Marg., ii, 286 Rob., ii, 286; Thos., ii, 286 Will., iii, 122

Peyver (Peyvre), see Pever

Pheasant, - , iii, I 8

Philibert, Hugh de, ii, 362

Philip, Alice, Lady, iii, 403, 429 Sir John, iii, 403, 429; Will., iii, 403

Phillips (Philips, Phillipps), Sir Edw., iii, 260; Edw., ii, 332 Fran., ii, 212 ; iii, 30, 201, 255 lirank, iii, 287; Jos., iii, 325 : Thos., iii, 325

Phillpotts, Jas. S., ii, I 49, 175, I 76 r 77 ; Hen., see Exeter, bps. of

Pick, see Pyke

Pickering, Dorothy E., iii, 259 : Sir Edw., iii, 46I ; Eliz., Lady, iii, 461 ; Eliz., iii, 428 ; Sir Gilb., iii, 46I ; Helen, iii, 428; Montagu, iii, 461 ; Thos., iii, 428 ; see also Puckering

Pickford, Thos., ii, $3 \mathrm{I}_{4}$

Pickle industry, ii, $\mathrm{x}$. 8

Pickman, Rich., ii, 222 ; iii, 273

Picks (Pykes), man. (Luton), ii, $350,355,363$

Pickshill (Stevington), iii, I02

Picot, Ad., iii, 46, $33 \mathrm{I}$; Anabella iii, 46, 33I; Bald., iii, 46, 234 235, 33I ; Dorothy, iii, 235 Isabel, iii, 235 ; Joan, iii, 45, 234 33I : Jolın, i, $325 n$; ii, 381 ; iii $46,234,235,330,331$; Laur. iii, 366 ; Mich., iii, 45, 46, 234 33 I Will., iii, 46 ; fam., iii, 206 ; see also Pigott

Pictavus, iii, 2

Picts Hill Gorse (Turvey), iii, IIO

Pierson, Thos., ii, 385 ; -, ii, I68 see also Pearson 


\section{A HISTORY OF BEDFORDSHIRE}

Pigeon, Edm., ii, 377; iii, 375 ; Walt., iii, $272,272 n$

Pigham, Will., iii, 2I9n

Pigott (Piggot, Pigot, Pygot, Pygott), Anne, ii, 338; Bald., iii, 15, 92, 296; Benj., ii, 338 Bridg., ii, 338 ; Dorothy, iii, I5 Eleanor, i, 390 ; ii, 2II, 224, 248 iii, 248 ; Eliz., ii, $224 n$; Fran. ii, 2 II, 22I, 224, 244, 248 ; iii, $248,258,259,449$; Hen., ii, I 79, I 80 ; iii, 292 ; Jane, ii, 385 Joan, iii, I5, 295 ; John, ii, 2 II 224 ; iii, I5, 215, 216 ; Laur. iii, 456; Lewis, ii, 21I, 224 Marg., i, 357 ; ii, 2 II ; Margery, iii, 259; Mary, ii, 338; Mich., ii, 2I I, 224; iii, I5, 295 ; Rob., iii, 449; Thos., ii, 211,224 ; iii, 227, $248,258,259,432,449$; Will., iii, 449 ; fam., ii, 204

Pilgrim, W., iii, 44

Pillage, man. (Marston Moretaine), iii, 3 ro

Pillinge field (Wootton), iii, 335

Pilling Grene (Wootton), iii, 329

Pilling-in-Wootton, man.(Wootton), iii, 332

Pilling Rowsberry, man. (Wootton), iii, 332

Pilling Shingay, man., see Pillingin-Wootton

Pilsworth, Edw., ii, 299

Pinch, mill of (Sharnbrook), iii, 9

Pinchgut Farm (Marston), iii, 307

Pinfold Hill (Ampthill), iii, 275

Pink (Pinke), Rob., ii, 162, 163

Pinkney, Agnes, iii, 7 I ; Sir Hen. iii, 7 I ; Hen. de, iii, 396,436 ; Isabella de, iii, 436; Rob. de iii, 436

Piozzi, Hester L., ii, $357 n$

Pipard, see Pippard

Pipewell Abbey (Northants), ii 275 ; iii, 24, 84 ,219, 221; abbot, i, $367 n$

Pipon, iii, 446

Pippard (Pipard), Ad., iii, 206 Ralph, iii, 16; Rob., iii, 206 Walt., iii, 206

Pippin Wood (Bletsoe), iii, $4 \circ$

Pirot, ii, $3^{82} n, 3^{8} 3$; iii, 196, 199 $246,247,248$

Pirot (Pyro, Pyrot), Elyas de, ii, 8I ; John, i, 37I $n$; ii, 380 ; Ralph, i, 366, $372 n$; ii, 3o, 32, $8 \mathrm{I}, 380$; iii, 5I, 54, I96, 248, 379 , 380,381 , 382 ; Reg., iii, 379 Rich., i, $372 n$; iii, 382 ; Will. i, 37 I $n$; ii, $377,379,380$; fam., ii, 324

Pirot's Manor, see Perots

Pirton (Herts), ii, 287

Pisaquila, see Puisaquila

Pisen, Rob., ii, 270

Pitchford, Admiral, iii, 90

Pittman-Coppin, Rev. John, ii, 3I7; iji, 406; John C., ii, 3I7; Mary, ii, 317 ; Susan, ii, 317

Pitt-Rivers, General, i, I75; ii, 2

Pitts, John, i, 399; iii, 22

Place, Will., iii, 49

Plantagenet, Anne, iii, I7 I Blanche, iii, Ior ; Edm., see Kent, earl of ; Eleanor, iii, I 7 I ; Hen., see Lancaster, earl of ; Marg., ii, 223; Rich., see York, duke of; Thos., ii, 223 ; see also Gloucester, dk of

Playdell, Will., iii, 3 го

Plenties, man. (Luton), ii, 350, 363,364

Pleshey (Essex), iii, I26

Plomer, John, i, 393 ; Sir Will., iii, 253; Will., ii, 2 I 2 ; iii, 253
Plumpton (Northants), iii, 700 Plunket, - , ii, I6 3

Pocklington, John, i, 337, 338; iii I 79

Podington, i, 305, 336, 337, 394 ii, 7I, II 5, 326; iii, $34,57,69$ 80-7, II7, II9, I22; adv., iii, 87 ; char., iji, 87 ; ch., i, 3 I $5 n$ 336 ; iii, 86 ; farms, iii, 80,81 ; man., iii, 82, 87, I05; man.-house, iii, 83 ; mills, iii, 82,85 ; placenames, iii, 82 ; sch., ii, 184 ; vicarage, i, 3I $6 n$

Podington, Lady Emma de, iii, 82

Podintone, see Podington

Podio Barsaco, John de, i, 327 iii, 408 ; Pontius de, i, $327 n$

Poer, John le, ii, 354

Poinz, Joan, iii, 268, 27I ; Nich. iii, 268, 271

Pole, Joan de la, ii, 227 ; John de la, ii, 227; Marg., de la, ii, 224, 227; Cardinal Reg., i, 333, 399 ; iii, 72 ; Rob., ii, 310; Will. (de la), ii, 224, 3Io; iii, 2I6, 403 ; fam., ii, 262

Polehanger, man. (Meppershall), ii, 290,291

Polehanger Farm (Meppershall), ii, 288, 291

Poley, Alice, iii, 246, 247; Fran. ii, 22I ; iii, 246, 247 ; John, ii, 204, 22 I; iii, 246, 247 ; Prudence, ii, 204; Will., ii, $204 n$; see also Pulley

Poleyn, see Pulleyn

Polge, Rebecca, iii, I65 ; Thos., iii, I65

Polhill (Polhill-Turner), Cecil H. iii, 203, 209, 2I 4, 21 5, 2 16; Fred., ii, $66 n$; iii, 20, 203, 215 ; Fred. C., ii, $66 n$; iii, 203, 2 I 5 ; Fred. E. F. iii, 215; John, iii, 203, 215 Nath., iii, 215; Miss, iii, 331 -, ii, 65 ; fam., ii, 66 ; iii, 182 , 202, 218

Political history, ii, I $7-72,83,95$, 102

Pollard, John, iii, 128

Poll-tax, ii, 90

Polluxhill, see Pulloxhill

Polochessele, see Pulloxhill

Polwarth, vsct., ii, 327 ; Amabel, vsctss., see Grey (of Wrest), Amabel, ctss. of

Pomfret (Pontefract), Geo., earl of, iii, 453 ; Thos., earl of, iii, 453

Pomfret, Eliz., ii, 363; John, ii, $363 ;-$, ii, 165

Pond's Close (Whiperley), ii, $36 \mathrm{r}$

Pont, Will., iii, 384

Pontefract, earls of, see Pomfret, earls of

Poole, Sir Arth., iii, 195 ; Benj., iii, 57 ; Joanna, I ady, iii, I95 Marg., iii, 57 ; Rev. R. B., ii, I7

Pope, Eliz., iii, I 4 o ; Sir John, iii, I 40 ; Sir Thos., ii, 374 ; iii, I4O Thos., ii, 357

Popham, Sir John, iii, 4 Io

Poplar Well (Blunham), iii, 228

Population, table of, ii, II 3-16

Port, Ad. de, i, 3I4 n; ii, 2I5, 218 ; Will. de, ii, 215, 216, 218

Porteous, Judith, ii, $216 n$, Rev. Sam., ii, 2 I6 $n$

Porter, Endymion, 347,382 Olive, ii, $347,2^{02}$; Sim., iii, I 72 ; Will., the, iii. 172 ; Miss, ii, I 77

Portland, Jer $\rightarrow$ me, carl of, iii, 277 Rich., eql of, iii, 277

Portland Cement Works, ii, 26I

Portmmin, Ld., ii, I91, I9?

Postuluma, Anne, iii, I98

$\mathrm{Pr}$ cantone, see Podington

40
Potsgrove, i, 370 ; ii, $30 n, 96, \mathrm{II}_{4}$, 346,$356 ; \mathrm{iii}, 336,343,368$, 421-3; adv., iii, 423; chant., iii, 423; char., iii, 423 ; ch., i, $3 \mathrm{I} 5 n$; iii, 423 ; farms, iii, $42 \mathrm{I}$; mans., i, 370 ; iii, 421 ; Nonconf., iii, 421 ; sch., ii, 181 ; iii, 421 ; vicarage, i, $316 n$

Pott, John, ii, $37^{8}$; Kath., ii, 378 ; Mary, ii, 378 ; Rog., ii, $37^{8}$

Potter, John, iii, 293, 372; Capt. Thos., ii, 69 ; Thos., iii, 293, 322 , 372 ; Will., ii, $24 \mathrm{I}$

Pottery, i, 273, 299, 300; ii, 314; iii, 82, 237: A.S., i, I 75, I 77 I78, I 79, I 86; British, ii, 8 ; iii 82; Bronze Age, i, 168, I69: Celtic, i, 170, I74; Roman, i I72, I 73, I 78, I 86; ii, 2, 4-15; iii, I 75, 280, 390 ; Rom.-Brit., i, 172,307 ; iii, 297

Potteshoe, Hen. of, ii, $289 n$

Pottingal, fam., iii, 407

Potton (Potone), i, I73, I74, 384: ii, I 7, 22, 93, 98, II $3,201,209$ 2 I $5,216,226,230,237-42,246$ 255 ; iii, 150, $269 n$; adv., ii, 239, 24I ; Brit. coins, ii, 237 , char., ii, 241 ; ch., i, $315 n, 326$; 333 ; ii, 239 ; coproite ind., ii, II 7 ; fire, ii, 239 ; mans., ii, 226, 237,$247 ;$ mkts. and fairs, ii, 88,239 ; mill, ii, 237, 239 ; Nonconf., ii, 24I ; place-names, ii, 237 ; racing, ii, I9I ; reptilian rem., $i, 34$; sch., ii, 184 ; Sun Inn, ii, 237 ; vicar, $i, 323,327 n, 34 \mathrm{I}$, $342 n$

Potton, Rob. of, i, 387

Potton Burdetts, man., ii, 2 Io, 238

Potton Much Manured, man., ii, 238 ; mill, ii, 239

Potton Rectory, man., ii, $238 n$, 239, 24 I

Potton Regis, man., ii, 237, 239

Potts, Anna L., iii, 25 I; John, iii 348 ; Nich., iii, 348 ; Spencer, ii, 43 ; Thos., ii, 45

Power, Geoff., ii, 248; Florence, iii, 126; Joan, i, 360; Nich., ii, 248; Sir Rich., iii, I26; Rob., ii, 322, 323 ; Sim., ii, 89

Powlett, see Paulet

Powney, fam., iii, 404

Poynder (Poynter), Thos., ii, 289 ; iii, 427

Poyntells (Arlesey), ii, 263

Poynter, see Poynder

Preaux, see Priaulx

Pratt, Edwin, ii, I 39

Prechier, Hugh le, iii, $364 n$

Prehistoric antiquities, $i, 7^{\circ}, I_{72}$ 174,270 ; ii, 281,314 ; iii, 76 , $350,390,439,448,45$ I

Prentis, Will., iii, 4 I 4

Presbyterians, i, 34O, 34I ; ii, 52 Prescott, Horace W., ii, 3I5; W. iii, 386

Prest, Hugh le, ii, 86 : iii, 165

Preston, Chris, 11, 299

Prester Deanery (Northants), ii, 189

Priaulx (Preaux), Drew des, ii, 321 ;.Isabel des, ii, $32 \mathrm{I}$; Matth., ii, I69, I70, I7I, I72; Mrs., ii, 172

Priest, Eliz., iii, 32, 238

Priestley (Flitwick), ii, 324 ; iii, $284,286,451 n, 453$; chap., iii, 288 ; man., iii, 286

Priestley Farm (Flitwick), Rom. coins, ii, 7

Priestley Moor (Flitwick), ii, I 35

Priestley Park (Flitwick), iii, 325 


\section{INDEX}

Priestley Plantation (Steppingley), iii, 324

Prigdale, iii, 251

Prikeavant, Will., iii, 73

Prior, see Pryor

Priory Farm (Harrold), iii, 66

Prisley, see Priestley

Proby, Hen., ii, 31 4

Profit, Miss, ii, 295

Prothero, R.E., iii, 149

Provenden, man. (Caddington), ii, 315

Pryor (Prior), Maj. Hen. M., ii, 277 ; John, iii, 212, 311; Maj. Ralston de V., ii, 277 ; Will., iji, 311

Pryors, Le (Le Monkes) man. (Turvey), iii, 113

Puckering (Puckeringe), Sir John, iii, 8; Sir Thos., ii, 226; see also Pickering

Puddington, see Podington

Puddle (Pudele, Podele), Hill (Houghton Regis), ii, 3, 102 ; iii, $355,389,390$

Puisaquila (Pisaquila), John, ii, 289, 290, 34I

Pulford, Rev. Josbua, iii, 416

Pulbanger, Ad. de, ii, $268 n$

Pullan, see Pulleyn

Pulley, John, ii, 65 ; iii, 18 ; see also Poley

Pulleyn (Poleyn, Pullan), John iii, 349; Rob., ii, 244; Walt., i, 404

Pullokeshull, see Pulloxhill

Pulloxhill, i, 370, 394 ; ii, 96, 114 $306,324,326,376-81$; adv., ii, 380 ; char., ii, 381 ; ch., i 3II $n, 314 n, 315 n, 371 n$; ii 332,379 ; dean of, i, 347; gold quartz, ii, $37^{6}$; inclosure, ii, 376 ; man., i, 370 ; ii, 376,378 80; place-names, ii, 376 ; sch., ii, 184 ; ship-money, ii, $37^{6}$; vicarage, $i, 316$

Pulloxhill, Hen. of, i, 366 : Steph. of, i, 366

Pulloxhill and Greenfield, man., ii, $376 n, 377 ; \mathrm{iji}, 380$

Pulloxhill Grange, ii, 377

Pulpits, ii, 206, 208, 218, 229, 269, $279,372,383$; iii, $26,27,56,68$, $75,127,141,164,167,178,233$, $250,278,283,340,410,414$

Pungiant, Rich., ii, 252, 253 ; iii, 260

Punters Stockings Close (Eversholt), iii, 377

Purchase, Laur., iii, 236

Purney, John, iii, I 34

Purvey, Alys, iii, 366; Eliz., iii, 366 ; Nich., iii, 366

Pusey, Phil., ii, I 26

Putnoe, man., see Puttenhoe, man

Putnoe Farm (Goldington), iii,

Puttenham, Mary, iii, 388

Puttenhoe (Putnoe), man. (Goldington), i, 365; iii, I 80, 202-4, 207

Puttenhoe Woods (Goldington), iii, 204

Putteridge Bury, ii, 196

Putteridge Park, ii, 359

Puttes, iii, $182 n$

Pycard, Joan, iii, 204; Marg., i, 390 ; Will., iii, 204

Pycot, see Picot

Pye, Anne, iii, 282 ; Edm., iii, 140 282 ; Hen., iii, 95: Hen. J., iii, 140 ; Rev. Jas., iii, I36 ; fam., iii 95

Pygeon, see Pigeon

Pygot, Pygott, see Pigott

Pygun, Will., ii, 36I $n$

Pyke (Pick), Ralph, ii, 8I ; Will., ii, 85
Pykenham, Will., iii, 408

Pykes, man., see Picks, man.

Pym, Capt. C. G., ii, 242 ; Eliz., iii, 166; Lt.-Col. Fran., ii, 70: Fran., ii, $66 n, 242,244$; iii, I66 ; Guy, i, 271 ; ii, 66n, 198 ; Will., ii, 244 ; iii, 166, 4II; -, ii, 197 ; fam., ii, 66

Pynders Hill, ii, 15I ; iii, 394

Pynfold, Marg., iii, 366; Rich., iii, 366

Pyres, Hen., ii, 378

Pyrley, Will. de, ii, 232

Pyro, Pyrot, see Pirot

Quadring, Alex., iii, 429 ; Alice, iii, 429; Marg., iii, 429; Rich., iii, 429

Quakers, see Friends, Society of

Quantock, Frances, iii, 443; John, iii, 443

Quarel, Isabella, iii, 120 ; Ivo, ii, 211 ; iii, $120 n$

Quarries, ii, 308,384 ; iii, $47,7^{6}$, $96,313,373,44^{8}$

Quatremars, Nich., ii, 238

Queensberry, duke of, ii, 192

Queen's Woods (Ampthill), ii, 145

Queldryck, Ad. de, iii, 418

Quince Hill (Old Warden), see Quint's Hill

Quincy, Arabella de, iii, 23 I $n$ Eleanor, ii, 351: Hadwisa de iii, 78, ror: Helen de, iii, 297; Marg. de, iii, 230 ; Rob. de, iii, I01, 297; Rog. de, $i, 361,388$ : ii, 351; iii, 101; Saher de, see Winchester, earl of

Quinny, John, iii, 434

Quinton (Northants), i, 338

Quint's Hill (Old Warden), i, 270, 271 ; iii, 252 ; Rom. encampment, ii, 13

Racc, Geo., ii, I9o ; John, ii, 190 Racing, ii, 190-5

Radcliffe (Radclyff, Ratcliffe), Alice, iii, 429; Delmé, ii, 193: Sir Edw., iii, 281, 304, 328 ; Edw., iii, 281, 293; Eliz., Lady, iii, 326 ; Farnaby, Lady, iij, 322 ; Frances, iii, 281 ; Fran. D., ii, 286 ; Fran. P., ii, 286 ; Hen. D., ii, 286 ; iii, 321 ; Sir Humph., iii, $28 \mathrm{I}, 283,293,303,304,328$; Isabel, Lady, iii, 281, 283, 304 Jane, Lady, iij, 304; John, ii 286,287 ; Sir Ralph, ii, 287 Ralph, iii, 321 ; Rob., iii, 283 429 ; Lady, iii, 158

Rademilde, Marg., iii, 194; Ralph iii, 194; Rob., iii, 194 ; Will., iii, 194

Radwell (Felmersham), ii, 324 ; iii 61 ; chap., i, 318; iii, 63; man. iii, 60

Radwell (Radewell), Alice de, iii, 60 ; Beatrice de, iii, 6o; Gilb. de, iii, 60; Hen. de, iii, 6o ; John (de), iii, 60, 61, 220; Marg., iii, 220; Margery, iii, 6r ; Petronilla of, i, 390 ; Rob. de, iii, 60 ; Thos., iii, 61, 220 ; fam., iii, 59

Radwell Hall (Felmersham), iii, 59

Raghton, Eleanor de, ii, 257 ; John de, ii, 257: Oliver de, ii, 257

Ragon, Eliz., iii, 106, I95; Sir John, iii, 106, 125, 195, 31 4 Kath., iii, 106 ; Reg., iii, 195. $3{ }_{4}$; Thos., iii, 106

Ragons Manor (Maulden), iii, 314

315
Rainald, iii, 146
Rainols, Ursula, ii, 94

Rainsborough, Col., ii, 52

Rake, - , iii, 358

Raleigh, John de, iii, 193

Ralph (Ralf), ii, 190; iii, 229, 245 375,419 ; archdeacon, i, 313; count, ii, 21 ; the hermit, i, 350 ; the steward, ii, 78

Ramerick, ii, 72

Ramridge End (Luton), i, 146; ii, 348

Ramsay (Ramsey), Rev. Norman, iii, 224, 226; Sir John, iii, 201; Will., ii, 332 ; Capt., ii, 48, 49

Ramsey Abbey, i, 312, 364 ; ii, 20, $21,76,86,207,209,215,237$, 261, 278, 294, 295, 296, 297, 299, $309,310,312,333,334,335$; iii, 9, 10, 12, 70, 128, 129, I 50, 199, $276,277,278,297,331$

Rand, John, ii, 287 : fam., iii, 59

Randall, Alex., iii, 105, 212 ; Anne, iii, 212; Will., ii, 245; W., ii, 198

Randei, Kath., iii, 6 I

Randes (Rands, Raundes), Edm., iii, 6r; John, ii, 267n; iii, II3; Oliver, iii, 61: Ricl., iii, II 5, I16

Randolph, Thos., ii, $235 n$; iii, $24^{8}$

Rands, see Randes

Rannulf, ii, 226, 227

Ransil Spinney (Podington), iii, 80

Ransom, E., iii, 297: W., ii, 5, 8, II, I3, I 4,41

Ratchford, Nich., iii, 246

Ratcliffe, see Radcliffe

Raundes, see Randes

Ravan, iii, 248

Raven, Dorothy, ii, $282 n$; Eliz., ii, 285; John, ii, 282, 285: Margery, iii, 456 ; Rich., iii, 456

Ravenhill, Agnes, iii, 396; Nich. iii, 97, 396

Ravensden, ii, II 3 ; iii, 180,183 , 209-14; adv., iii, 214; char. ii, I $84 n$; iii, 214 ; ch., i, I 40 , $315 n$; iii, 213; dean of, i, 347 ; mans., i, 365; iii, 203, 210-12 ; place-names, iii, 210 ; sch., ii, 184 ; vicarage, $i, 316 n, 317$

Ravensden, Nich. de, iii, 210

Ravensden Grange (Manor), iii, 209, 210, 211

Ravensden House, iii, 209

Ravensholt Grange (Old Warden), i, 364 ; iii, 247, 253

Rawlins (Rawlyns), Agnes, iii, 210 ; Fran., iii, 211 ; Gabriel, iii, 407 ; John, iii, 210, 21I; Rob., iii, 211 ; Steph., iii, 210, 211 ; Thos. ii, 292 ; Will., iii, 407

Raworth, Rob., ii, $204 n, 286$

Ray, Rich., iii, 187

Raymond, Thos., iii, $\mathrm{ro}_{4}$

Raynar, iii, 104

Raynsford, Frances, ii, 282 ; Maj Gen. Hanbury, ii, 282, 285 ; Geo. N., ii, 282 : Thos. A., ii, 282,285 ; fam., ii, 285

Raynton, Geo., ii, $293 n$

Reach, man. (Leighton Buzzard), iii, 403

Read (Reade, Rede), Alex., iii, 18 , 438; Alice, iii, 246; Eliz., ii, 323 ; iii, 244 ; Innocent, ii, 323 ; iii, 244; Jas., iii, 349; Joanna, iii, 438 ; Rob., iii, 246 ; fam., iii, 247

Reading (Berks), A.S. rem, i, 184 sch., ii, 159

Reading Abbey, ii, 23; iii, 289, 294 Readshill Plantation (Maulden), iii, 313

Reckey (Riky), Barth., ii, 289, 290, 34 I 


\section{A HISTORY OF BEDFORDSHIRE}

Rectory Manor (Northill), see Northill College Manor

Redbornestoke hund., ii, II5; iii, 267-335

Redburn, Sim. of, i, 386

Reddall, see Riddell

Reddings, man., see How End

Rede, see Read

Redeware, Rob. de, iii, 196

Redinges, Rob. de, iii, 276

Reeve (Reve, Rowe), Agnes le, iii II 4 ; Alice le, iii, II 4 ; Geo., ii, I64; Hugh, i, 337, 338, 340, 342 ; iii, 273 ; Joanna le, iii, II 4 ; John le, iii, II 4 ; Thos., ii, 164,218 ; iii, IOI, 392

Regine, man. (Cardington), see Fenlake Barns

Reincourt, Rich. de, ii, 247

Rekesduna, see Roxton

Religious houses, i, 349-404

Relye, see Thurleigh

Remigius, bp. of Lincoln, see Remy

Rempston, Sir Thos. de, iii, 263

Remy (Remigius), bp., see Lincoln bps.

Renhall, see Renhold

Renhold, i, $335 n, 365$; ii, I1 3, I54 ; iii, I80, I83,202, 21 4-18; adv. iii, 218; Chantrie Close and lands, iii, 217 ; char., ii, $184 n$; iii, 218: ch., i, $315 n, 325 n$ 380 ; iii, 217 ; earthworks, i, 28o, 284,285 ; man., iii, 13, 215 ; Nonconf., iii, 214 ; place-names, iii, 215 ; sch., ii, 184 ; vicarage, i, $316 n$

Renhold (Ronhale), Rich., iii, 216 ; Sybil de, iii, 215

Repingdon (Repyngton), Philip, bp., see Lincoln bps.; Will., iii, 442

Reptiles, i, 102-3

Reptilian remains, i, 34-5

Repton, G. W. J., iii, $74 n$

Repyngton, see Repingdon

Resby, Will., iii, 250

Restwold, Ralph, iii, 246

Retchford, Nich., ii, I 80

Reve (le), see Reeve

Rewse, John, ii, I67; see also Ros and Rous

Reynal, John, iii, 384 ; John S. N., iii, 384

Reynardson, Allan, iii, 198; Anne, ii, 317 ; John, ii, 3 I 7

Reyner, Cuth., iii, 259; John, iii, I 37

Reynes, man. (Stanbridge), see Morteynes

Reynes, Alex. de, i, 362, 365 . Amabel, iii, 152 ; Cecilia, iii, 150 ; Eliz., iii, 309, 312, 345, 385; Joan, iii, I50 ; John, iii, I 50 309; Nath., iii, 345 ; Ralph, iii, I50, I52: Rich., iii, I 50, 309 ; Sibyl de, i, $362 n$; Sir Thos., iii, II 3, I47, 309; Thos., iji, I50, 309, 3II, 3I2, 4I 7 ; fam., iii, 313

Reynolds (Reynold), Edw., iii, 5I ; Frances, iii, 5I ; Fran., iii, $5 \mathrm{I}$. John, ii, 245 ; iii, 233 ; Walt., iii, I95: -, iii, 4I 4

Rhee, riv., ii, 7I, 218,230

Rhodes, Sam., iii, 287

Rbopalocera, i, 79-8o

Rice (Ryce), Geo. R., iii, 45; Will., iii, 106

Rice-Trevor, Elianore M., iii, 49; Miss, ii, I8I $n$; iii, 45

Rich, Sir Thos., ii, I73; Thos., iii,

Richard, archd. of Beds., i, 3I 3

Richard I., king, iii, 229
Richard II., king, ii, 2II, 235

Richard, king of Germany, ii, 384 ; iii, 353

Richards, Chas., iii, 274; John, ii, 374, 375: Sam., iii, I 60

Richardson, Alice, ii, 329, 330 ; Rev. John, ii, 220 ; Pet., ii, 330: Will., ii, 329, $33^{\circ}$; Will. S., iii, $326 ;-$ ii, 222

Richborough (Kent), i, I 84

Richmond, earls of, iii, 298 ; Hen., iii, 230 ; John, iii, 297

Richmond, Pet. de, ii, 234

Richmond and Derby, Marg., ctss. of, ii, 256

Riddell (Reddall, Ridel), Agnes, iii, 223, 224; Amb., ii, 69; iii, 310, 423, 436; Eliz., iii, 423 ; Jas. B., ii, 383; Jane, ii, 385; Sir John, ii, 385 ; John, iii, 224 ; Sir John B., ii, 385 ; Ralph (Ralf), iii, 219, 223, 224; Thos., iii, 310; - iii, 375

Riddises Spinney (Lidlington), iii,

Ridel, see Riddell

Ridgeway, Col., ii, 195

Ridgmont, i, 336 ; ii, 87 , I15, I 29, I 40, I4I, I 46, I47; iii, 267, 320$323,394,459$; adv., iii, 323 ; char., iii, 323; ch., iii, 323: crown property, iii, $27 \mathrm{I}, 322$; man., iii, 321; Nonconf., iii, 320: place-names, iii, 321 ; Rom. rem., iii, 320 ; sch., ii, I8.4; wells, iii, 320

Rievaulx, Sylvan, abbot of, i, $353 n$

Rigge, Isabella, ii, 224 ; : Rob., ii, $224 n$

Riky, see Reckey

Ringe, Thos., iii, 277

Ripley, Rev. Thos., ii, 280

Risceby, Alice, ii, 334; John, ii, 334 ; Will. de, ii, 334

Riselai, see Riseley

Riseley, i, 394: ii, II5; iii, 40, 43 , I06, I 23, I 43, I 57-61 ; adv., iii, I6I : Cake Bread Close, iii, I6I ; char., iii, I6r ; ch., i, 315 n, 333, 394 ; iii, 160; deanery, i, 347 ; ind., iii, I 57 ; man., iii, 134,157 ; mills, iii, I57; moats, iii, I57; Nonconf., iii, I 57; place-names, iii, I57; sch., ii, I84; wells, iii, I 57

Riseley (Risely, Risley, Rysley), Geoff. de, iii, I 58, I 59; Hen de, iii, I59; Hervic de, iii, I06; Jas. iii, 328; John (de), i, 387 ; iii, 24, I 59, 244; Lawr. de, iii, I58; Rich. de, iii, I59; Walt. de, iii, I58; Will. de, iii, I 58

Riseley Lodge (Riseley), iii, I 57

Riseley Old Domain, earthwork, i, 306

Risinghoe (Goldington), iii, 204, 207 Risinghoe Castle (Goldington), i, $285 n, 296,297$; iii, 202, 206, 207 Risley, see Riseley

Rivers, earls, iii, I 18 ; Ant., iil, 1 I 8 Rivers, Marg., iii, Io: Rich., iii, 92 Rixband, Marg., ii, 2 I I Will., ii, 2 II

Roads, Brit., iii, 350 ; Rom., i, I 76, 269, 27x $358,37 \mathrm{I}$; ii, I-8, I7, I8, 23, 40, 46, 98, 203, 209, 212, 223, 276, 280, 300, 31 4, 31 7 : iii, $256,350,355,383,389,390$, 400

Roais, ctss., see Beauchamp, Rohesia

Robbins, see Robins

Robert, iii, 92,238

Robert Bruce (king of Scotland) iii, 299
Roberts, John, ii, 3 ro

Robins (Robbins), E. C., ii, I 76 : John, ii, $378,379,380$; iii, 27 Mary, iii, 27 ; Susanna, iii, 27

Robinson, Dorothea, Lady, iii, 434; Dorothea, iii, 434; Frances, iii, 244 : Sir Geo., iii, 434 ; Gilb. iii, 84 ; Rev. John, ii, 333 ; John ii, I67; iii, II 7, 243, 244, 434 Marg., ii, 353: Underhill, iii, I 44 Will., ii, 30I ; iii, I22; Dr., ii, I98; Mrs., iii, $386 ;-$, iii, 355

Roche, fam., iii, 48

Rochechouart, Guy de, ii, 35I Sybil, ii, 35I

Roches, John des, ii, 309; iii, 315 Pet. des, bp., see Winchester, bps.

Rochesdon, see Roxton

Rochester, bar. of, iii, 377, 419

Rochester, Ansgot of, iil, 376, 377, 419: Paris, archd. of, ii, 320

Rockingham Castle (Northants), ii, 22 ; iii, 70,82

Rodentia, i, I4I-3

Rodland, see Rowland

Roe, Henricus O., ii, I $84 n, 304$ see also Reeve and Rowe

Roger, i, 358 ; ii, 376 ; iij, 165,258 259, 29I

Rogers, Alice, iii, I98, 3 II : Hen. iii, I98; Jas., ii, 380 ; Rich., i, 387 ; Rog., ii, 222 ; Sam., iii, 317 W. H., iii, 207 ; -, ii, I69, I 70

Roherd, iii, 344

Rohrweger, Miss, iii, 329

Rokele, John de la, i, 400

Rokes, Eliz., ii, 329, 330 ; Rich., ii, 329,330

Rokesdon, see Roxton

Rolffe, Will., iii, 260

Rolland, iii, 247

Rolt (Rolte), Anna, iii, I 83 Capt. Edw., iii, I55; Edw., ii, 42 ; iii, I55; Eliz., iii, 224 ; Frances, ii, 280 ; Fran., ii, 280 . Sir John, iii, $1_{4} 6$; John, iii, $I_{4} 6$, I83, 224; Judith, iii, I 46,183 ; Mary, iii, I 55 : Sam., iii, 20 , 146 . Steph., iii, I 48 ; Susannah, iii, I 48 ; Sir Thos., iii, I 55 ; Thos., ii, 44 ; iij, I $45, I 46, I 48, I_{54} n, I 55$, I83, 2 II, 224, 226 ; Walt., ii, 277 280 ; iii, I $54 n, 155$; Will., iii I $46 n, 259 ;-, i, 342 n$; fam. iii, I49, I56

Romano-British period, ii, I-I

Roman remains, i, $186,271,274$ ii, 6,7, I2-1 5, 21 2, 223, 294, 344 iii, $44,82,175,243,252,257$, $262,280,320,390,402$; see also burials, coins, implements, ornaments, pottery

Romylo, Steph., iii, 216

Ronbale, man. (Ravensden), see Ravensden, man.

Ronhale, par., see Renhold

Rook, Admiral, iii, 4

Rookham, Rob., iii, 396

Rooktree Farm (Holcot), iii, 386

Rookwood, Amb., iii, 354

Roos, see Ros

Rootham's Green (Colmworth), iii, 186

Roper, Kath., iii, 443; Will., iii, 443

Ros (Roos), Ralph de, iii, 36 ; Rob. cle, ii, 3 I $n$; Serlo de, iii, 36,37 , $44 n$; Will. de (le), i, 38I; iii 219; fam., iii, I 49; see also Rewse and Rous

Rose, man. of the (Lond.), ii, I59

Rose, E. P., ii, 209, 222 ; Thos., i 333 ; archd., iii, 295 ; Mrs., iii, 364 


\section{INDEX}

Rosewell, Pet., iii, 145

Roses (Rosae), i, 55

Rotherham, college at, ii, 157

Rotherham, Agnes, ii, 363 ; iij, I3, 313; Alice, Lady, iii, 292 ; Alice, ii, 295, 299, 300; Anne (Ann), ii, 343,380 ; Eliz, ii, 363,375 : Geo., ii, $40 n, 295,305,342,343$, $353,356,357,358,361,363,372$, 380 ; iii, $13,293,300,415,432$; Isaac, ii, 343 , 380 ; Jane, iii, 443 ; Sir John, ii, 353, 356, 363, 364, 372 : John, ii, 219, 295, 352, $354 n, 356,359,363,374,380$; iii, 3 г 3 ; Nich., ii, $363 n$; Sir Thos., ii, I5o, 354, 372 ; iii, 292 , 299, 30o, 437, 443 : Thos., ii, 219, 295, 352, 353, 358,360, 361, 363 ; iii, 299, 437 ; Thos., archbp., see York, archbps. of, and Lincoln, bps. of ; -, Lady, ii, 372 ; fam., ii, 307

Roubury, Gilb. de., i, 327

Round Green (Luton), i, 146 ; iii $34^{8}$

Round Hill (Roxton), i, 297

Round Wood (Sharnbrook), iii, 89

Roupe, Thos., iii, 436

Rous (Rouse, Rowse), Eliz., iii, I59 Geoff. le, iii, 332 ; Joan le, iii, 332 Rich. le, iii, 137; Rob., i, 397 Rodenham, ii, 313, 384; Sim. le iii, 332 ; Thos. le, iii, 162; Will. iii, I29, 332; Admiral, ii, I94 Mrs., iii, 129; see also Rewse and Ros

Rowe, John, iii, 129 ; Nich., ii, 206 ; Thos., iii, I29, 13I, 151; see also Reeve and Roe

Rowington (Warws), iii, $294 n$

Rowland (Rodland), Nich., iii, 241 Pernel, iii, 24I ; Walt., iii, 24I fam., iii, $24^{\circ}$

Rowlands, man., (Cople), iii, 182, 240

Rowley, Agnes, ii, 325

Rowney, i, 365; iii, 227, 259

Rowney Grange, i, 365; iii, 259

Rowney Warren, ii, 1477 ; iii, 256

Rowse, see Rous

Rowthe, Thos, ii, 157

Roxhill (Wroxhill) (Marston More-

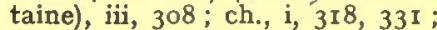
iii, 3 I 3 ; man., iii, 3 ro

Roxhill Farm (Marston Moretaine), iii, 310

Roxox, see Ruxox

Roxton, ii, 113 ; iii, I 75, I 76,180 186, 193, 198, $218-22,384$; adv. iii, 222 ; char., iii, 222 ; ch., i, $315 n, 382,384$; iii, 22I ; earthwork, i, 297, 302; man., iii, I83 219, 221 ; mill., iii, 221 ; Nonconf. iii, 218 ; sch., $\mathrm{ii}, \mathrm{I} 84$; vicarage, $i$, $316 n$

Roxton (Rokesdone), Alice de, iii, I92 n; John (de), i, 377: iii, $192 n, 364$

Roxton Wood End, man., see Carlyles

Roys, ctss., see Beauchamp, Rohesia

Royston (Herts), iii, I97 $n$; ii, I9I ;

iii, $197 n$

Rualon (Ruallon), ii, 376 ; iii, $18 \mathrm{r}$, 184

Ructona, see Roxton

Rudde, Thos., iii, 85: Will., iii, 85

Rudge, Susanna, iii, 427

Ruding (Rudyng), Hen., i, 398 John, ii, 213

Rudlonds (Rudlandesfelde), man. (Cranfield), iii, 277

Rudyerd, Sir Benj., iii, 427

Rudyng, see Ruding
Rufford, Eleanor, iii, 453 ; Joan, iii, 422 ; John, iii, 422, 423, 453 Thos., iii, 422, 439, 453

Rufinus, i, I 73

Rufus, Agnes, ii, $301 n$; Godf., iii 238; John, iii, 238 ; Laura, iii, 239: Rob., ii, 301 ; iii, 159, 238 . Sim., ii, 301 ; iii, 238, 239 ; Will., ii, 301 ; iii, 240

Rugemont Castle (Ridgmont), iii, 321,322

Ruggerwyk, Margery, iii, 195 : Thos., iii, 195

Runhale, see Renhold

Rupert, Prince, ii, 46, 50 ; iii, 4

Rushden (Northants), i, $376 n$. ii, 71 ; iii, $34 n, 40,117$

Rush-matting ind., i1, $124-5$

Rushmere Lodge (Leighton Buzzard), iii, 400

Russell, Cath., Lady, ii, 277 ; Eliz., Lady, ii, $277 ;$ iii, 332,376 Marg., Lady, iii, 67: Rachel, Lady, ii, $57 n$; Lds., iii, 461 . Chas., ii, 65, 188; Chas. J., ii 187; Edw., ii, 6r ; Fran., ii, 244,277 ; iii, $67,203,332$; see also Bedford, earl of ; Geo. W., ii, 65 ; iii, 20; John, ii, $39,65,66 n$, 7o; iii, 266, 459; Will., ii, 56, 57 , $60,62,277$; iii, 332,460

Russell (Russel), Arth., see Ampthill, Ld. : Sir Edw., iii, 437 Admiral Edw., ii, 6o; Edw., ii, $43,54 n$; iii, 376 ; Fran., iii, 376 ; Sir Geo., ii, 41 ; Geo., ii, $65 n$ Hawise, iii, 449, 456; Herbrand A., see Bedford, dk. of ; Isabel, iii, 30 I ; John, ii, 43,54 ; iii, 376 ; I.etitia, iii, 376 ; Matilda, iii, 301 ; Ralph, iii, 301 ; Sir Thos., iii, $43^{\circ}$; Thos., ii, 213, 245; iii, 37, 143 156, 200, 218, 233, 31 5, 334 Sir Will., iii, $376,429 n$; Will. iii, $334,376,449,456$; 一, ii, 303,312 ; iii, 108, 454 ; fam., ii, 56 ; iii, 20, 324, 436, 459

Russell Park (Bedford), i, 186, 190 $285 n$; iii, 5

Russell's Close (Ampthill), iii, 275

Ruthyn, Rich, de, iii, 46

Rutland, dk. of, iii, $45^{8}$

Ruxox (Flitwick), i, 305, 375; ii, 83,85 ; chap., i, $37 \mathrm{I} n$; iii, 284 286 ; man., iii, 286

Ryce, see Rice

Rycroft, - , iii, I95

Ryde, Hen. de, ii, 328; Will., ii, 328

Rydemounde, see Ridgmont

Ryder, John W., ii, 256; Nath., iii, 310 ; Thos., ii, 257

Rye, La, bar., iii, 428

Rye, Agnes de, iii, 426; Alina de, iii, 426; Hen. de, iii, 426 ; Hubert de, iii, 426; Isabel de, iii, 426

Ryggley, Nich., iii, I 45

Ryman, Will., ii, 378

Rysley, see Riseley

Sabine, Eliz., ii, 337 ; Sir John, ii, 337

Sacchevilla, see Sackville

Sacheverell, Rich., iii, 230

Sachynton, Rob. de, iii, 276

Sackville (Sacchevilla), Alex. de, ii, 320 ; Athelina, iii, I59 ; John, iii, 158 ; Thos., iii, $159 n, 322$; iii, 158

Sacombe Park (Herts), iii, 155

Sadleir, Edw., iii, 340 ; Ralph, iii $34^{\circ}$; Rich. V., iii, 340 Thos. L.,
Sadleir (cont.)

iii, 340; Ursula, iii, 340; Will. iii, 340

Sadler, Mich., F. iii, 9 : Ralph, ii 256 ; Thos., ii, 44

Saffey (Saffrey), Alice, ii, 322 ; Brian, ii, 75, 322 ; Joan, ii, 322 Margery, ii, 322 ; Thos., ii, 322

Saher (Seiher), ii, 326; iii, $32 \mathrm{I}$

St. Albans (Herts.), i, I, $3,324 n$ $372 n$; ii, $314,350,356,358,363$. iii, 10, 362 ; almshouses, iii, 333 battle of, ii, 37,352 ; sch., ii, I 78

St. Albans, Greg. of, i, 353

St. Alban's Abbey, i, 310,313 n, 314 $323,350-4,358,360,363,366$ 386 ; ii, $23,35,270,321-5,342$ $36 \mathrm{I}, 362,368,369,373,374,378$, 384 ; iii, I6, I48, 149, I8I, 344 $350,370,393,394,401,422,426$, 430, 43r, 448; abbot of, Geoff., i, 35 I $n, 358$; ii, I 78,36 I $n, 363$. Paul of Caen, i, $3^{\text {ro }}$; Rich., ii, 178,361 ; Rob., i, $359 n$; cell of, at Millbrook, iii, 316,318

St. Amand, bar. of, ii, 324

St. Amand, Eleanor, Lady, ii, 147 Eliz., iii, 140, 380; Lds., ii, 277 ; iii, 187,317 ; Almaric, iii, 187 ; Rich., ii, 337 ; iii, I40, 187,230 380 ; Will., iii, 230,380

St. Amand, Almaric (Aimery Aumary), de, i, 352 ; ii, 32, 33 $324,342,376,379$; iii, 236,252 , $271 \mathrm{I} n, 272,292,3 \mathrm{I} 7,3$ I 8,379 380, 440 ; Ant., iii, r 40 ; Asseline (Azeline), ii, 324; iii, 272, 317; Eleanor, iii, 272, 292, 317, 379 Sir Ernery de, iii, 2 : Emery de, iii, 2, I 13,324 ; Joan de, iii, 236 ; John de, iii, 252, 272, 317, 379 ; Mary de, iii, 440 ; Ralph, ii, 2 II, 324 ; iii, 236, 237, 272, 31 7 ; fam., iii, 326

St. Andrew's Priory (Northampton), i, $315 n$; iii, 23

St. Anne's Farm (Thorncote), iii, 244

St. Anne's Hill (Luton), ii, 348

St. Bartholomew's Priory (Lond.), iii, $45^{6}$

St. Briavells, Will. de, iii, 281

St. Bridget's Well (Houghton Regis), iii, 390

St. Clare (St. Clair), Gunnora de, iii, I9o; Hamo de, iii, Igo; Jas., iii, 223; Marg., iii, 433 ; Phil., iii, 433

St. Croix, Hugh de, ii, $32 \mathrm{I}$ iii, $27 \mathrm{r}$; Isabel, ii, 321 ; iii, 271 ; John de, iii, 272 ; Pet. de, ii, $277,321,383$; iii, $27 x, 272,318,346$; Rob. de, ii, 321 ; iii, 272 , 318 ; Thos. de, iii, 272

St. Edmunds Abbey, i, 312, 362 ; ii, I8, $25 n$; Baldwin, abbot of, iii, 38

St. Edward, Alice, iii, 33I ; Hugh, iii, 33I, 333; Joan, iii, 33I ; John de, iii, 220 n: iii, 33 J

St. Evroul's Abbey, iii, 294 n

St. Faith's Priory (Longueville), iii, $3{ }^{15}, 343 n$

St. Francis's Home (Campton), ii, 266

St. George's Chapel (Oxford), iii, I $53 n$

St. George's Chapel (Windsor), iii, $366,401,403,404,409,429$

St. Giles Hamstead Priory, 1ii, 385

St. Giles in the Wood, convent, (Flamstead), i, $315 \%, 375$; iii,

St. Gilles, Eustace de, iii, $60 n$ 


\section{A HISTORY OF BEDFORDSHIRE}

St. Helen's Priory (London), i, $315 n ;$ ii, 23r, 232, 233

St. James's Abbey (Northampton), iii, $67,109,114$

St. John, Ladies, iii, $6 \mathbf{r}$; Agnes, ii, 243 ; iii, 257, 396; Arabella, iii, I37; Dorothy, ii, 277; Emma M. E., iii, 143, 160 ; Frances, iii, 43; Kath., iii, 137: Lds., i, 339 ; ii, $40,63 n, 70,130,146$, 147, $183 n, 197,257$; iii, 3,35 , $40,4 \mathrm{I}, 42,6 \mathrm{I}, 73,94, \mathrm{I} 23, \mathrm{I} 33$, $134,142,143,155,158,160,165$, 175, I80; Beauchamp M., iii, $4 \mathrm{I}$; John, iii, 41, 137, I 72, 258, 259 ; Oliver, ii, $44,56,277,323$; iii, $35,94,109,123,125,137,146 n$, $158,163,179,180,194,195,257$. 258 ; Paulet, iii, I77; Rowland, iii, $4^{\mathrm{I}}$; St. Andrew, iii, 4I, $\mathrm{I} 63$ : St. Andrew B., jii, 163 ; Will., iii, 4 r ; Will P., ii, 243

St. John, Agnes, ii, 277, 323; iii, 194; Sir Alex., ii, 40; Alice, iii, 343 ; Anne, ii, 252 ; Ant., ii, 252 ; Sir Beauchamp, ii, 4I, 42, 44 ; iii, 163, 172; Rev. Edm. T., iii, 42 , 43; Eliz., iii, $73,343 n$; Fran., ii, 207 ; Sir Hen., iii, I63 ; Hen., i, 396 ; Sir John, ii, I 50, 277 ; iii, $7,43,60,125,127,137,158,159 n$, I68, 197, $343 n$, 396; John, i, 395 , 396 ; ii, 39, 296; iii, $4^{1}$, 125,137 : Judith, iii, 395 ; Marg., ii, 277 ; iii, 4 I, 137 ; Margery, ii, $385 n$ iii, 343 ; Mary, ii, 207 ; iii, 380 ; Sir Oliver, iii, $4 \mathrm{I}$; Oliver, i, 397 ; ii, $39,55 n, 277,296,323,385 n$; iii, $137,143,146,160,177,194$, 395, 396; see also Bolingbroke, earl of; Sir Paulet, iii, 4I, I37, 163, I77; Pawlett, ii, 56 ; Sir Rowland, iii, 73 ; St. Andr., ij, 64: iii, I 72 ; Sir Walt., ii, 207 ; Will., iii, 74, 380

St. Johns, man. (Kempston), sec Brucebury

St. John's College (Cambs.), i, 346 ; ii, I 57, r64, I66, 168, 293; iii, 69, 230, 296, 31 3

St. John's College (Oxford), ii, I59, $25^{\circ}$

St. John's Hospital (Bedford), i, $316 n, 396-8$; iii, 31, 225

St. John's Hospital (Hockliffe), i, 4 OI-2

St. Leonard's Hospital (Bedford), i, 354, 398-9; iii, 31, 237; adv., i, 398 ; iii, 22 ; chant., i, 326

St. Macute's Chapel (Haynes), i, $318 n, 35$ I $n, 352$; ii, 344 ; man., ii, $342-3$

St. Macute's Wood (Haynes), ii, 343

St. Martin-le-Grand, ch. (Lond.), i, $364 n$

St. Mary Colechurch, ch. (Lond.), i, 392

St. Mary de Pré, abbey (Leicester), i, $315 n ; \mathrm{iji}, 94$

St. Mary Magdalene's Hosp. (Dunstable), i, 400-1; iii, 364: masters, i, 40

St. Mary Magdalene's Hosp. (Luton) i, $399-400$

St. Mary Over (Dunstable), iii, 364

St. Mary's (Dunstable), ii, 7

St. Mary's Abbey (Stratford), see Stratford Abbey

St. Maur, Mary, Lady, iii, $440 n$; -Rich., Ld., iii, $440 n$

St. Maur, Alice, iii, $440 n$; Ellen de, ii, 328; Nich., ii, 224,328 ; see also Seymour

St. Mildred, Wallbrook, ch. (Lond.) i, 392
St. Neots (Hunts), ii, 214 ; iii, 189 St. Neots, Hen. of, i, 353; W. de, i, $327^{n}$

St. Neot's Priory (Hunts), i, $314 n$, $394 n$; ii, 226, 228, 229, 25r, $253,254,262$; iii, IIO, II 3 , II 6 II $7, x_{42}, 184,190 n, 196,221,231$

St. Nicholas' Abbey (Angers), ii, 281

St. Nicholas' Abbey (Arrouaise), i, 387,388 ; Gervase, abbot of, i, $387 ;$ iii, 65

St. Oswald's Priory (Glouc.), i, $383 n$

St. Paul's Cathedral (Lond.), i, 312, $317,324 n, 35^{8}, 360$; ii, 71,153 $314,315,316,3$ I $8,319-20,35$ I $n$ 356 ; iii, 140,206 ; deans, ii, 318 , 320 ; iii, 230,233

St. Peter's Abbey (Dorchester), i, $3^{8} 7 n, 388 n$

Saintpierre, Isolda, iii, $452 n$

St. Remigius, see St. Remy

St. Remy, Agnes de, iii, 223, 224 Cecilia de, iii, 223, 224: Elena de, iii, 223 ; Rich. de, iii, 223, 225 ; Rob. de, iii, 126, 223, 224, 225 ; Will. de, iii, 223

St. Sampson, abbot of, iii, 119n St. Sampson, Joan de, iii, I 19 Ralph de, iii, 119,163

St. Sepulchre's schs. (Lond.), iii, 368

St. Stephen Jewry, ch. (Lond.), i

St. Thomas's Chapel Manor (Meppershall), i, 391 ; ii, 288, 289 290, 305, 34I

St. Valery (St. Walery), Aubrey de i, 379; Guy de, i, 379; Reg. de, ii, 283 ; iii, 339

Salchou, see Salphobury

Salden House (Bromham), iii, 45

Sale, Nich. de, iii, 22 ; Walt. de la, iii, $\mathbf{r} 66$

Saleby, Rob. de, iii, I34

Salem (Solemn) Thrift (Bromham), iii, 44

Salford, ii, 45, II4: iii, 336,388 , 424-5; adv., iii, 425 ; char., ii, I $84 n$; iii, $425 ;$ ch., i, $315 n, 333$, $378 n$; iii, 424 ; man., iii, 424 ; mill, iii, 424: Nonconf., iii, 424 ; iii, 425 ; sch., ii, 184 ; vicarage, i, $316 n$

Salford, Dan. R., i, 367n, 368, 369 ; Hugh de, ii, 3or ; iii, 424 ; John de, iii, 424 ; Nigel de, ii, 301 ; iii, 424, 425 ; Pet. de, iii, 424 ; Rog. de, $i, 371 n, 378,379$

Salinis, Steph. de, ii, 255

Salisbury, Will., earl of, ii, 27, 244 ; iii, $184,439 n$

Salisbury (Salusbury), John, ii, 69: Rev. Lynch, ii, 310 ; Rog. of, i, $353 n$; Sarah, ii, 3 ro ; Sir Thos., ii, 310: Will, iii, $6 \mathrm{I}$

Salmon, Eliz., ii, 292 ; Thos., ii, 292 ; - , ii, 133,134

Salndy, see Sandy

Salomon, priest, iii, 107

Salph End (Renhold), iii, I80, 21 4-16

Salphobury (Salpho), man. (Renhold), i, 380 ; iii, 210,2 I6

Salter, Geo., ii, 256

Saltmers, Pet. de, iii, 186

Salusbury, see Salisbury

Salvation Army, ii, 214 ; iii, 3I, 367

Salveyn, Gerard, ii, 354

Salvho, see Salphobury

Sambrook (Sambroke), Eliz., iii, 177; Sir Jeremy V., ii, 62 ; iii, 105, 109, 137, 177, 179; Judith, iii, I05, I 77 : Susannah, iii, 177
Samian pottery, see Pottery, Roman Sampson, Humph., ii, 268; Jas., ii, 268; John, iii, I 45 ; Laur., ii, 268

Samshill (Lower) Farm (Westoning), iii, 45

Samshill (Upper) Farm (Westoning), iii, 45I

Samwell, Anne, ii, 346; Ant., ii, 346 : Arth., ii, 346: Will., ii, 346 Sandall, Mrs., ii, 222

Sandeia, see Sandy

Sanders, see Saunders

Sanderson, Anne, iii, 205, 209 John, iii, 205; Thos. A. E., ii, I 80 ; see also Saunderson

Sandon, John de, ii, 359

Sandwich, earl of, ii, $64 n$; Edw. Montagu, earl of, ii, 339 .

Sandy, i, 271, 272, 273, 274, 384 394: ii, 66, 107, I13,116, 199, $201,226,228,237,239,242-6$, 248,255 ; iii, $190,227 n, 243$ 264 ; adv., i, 383 ; ii, 245 ; A.S. rem., i, I84, I89; ii, 242 ; chant. ii, 245 ; char., ij, 246 ; ch., i, $315 n, 325 n, 332 n, 382,384$ : ii, 244 ; coins, i, I 73, I 74 ; ii 2, II, 242 ; earthworks, ii, 242 ; Hasells, the, if, 242,244 ; inclosure, ii, 97 ; inds., ii, 117,118 mans., ii, 243; mkt. gardening, ii, I38, 1 39 ; mills, ii, 243 n, 244 Nonconf., ii, 245 ; place-names ii, 242 ; prehist. rem., i, 166 rectory, ii, 242 ; rent, ii, I 32 Rom. rem., ii, I, 9-II ; sch., ii 184,242 ; stone quarry, ii, 242

Sandy, Hen. of, ii, 279; Hugh of ii, 279

Sandy Place (Sandy), i, 300 ; ii, 242 Sandyplane Plantation (Husborne Crawlev), iii, 394

Sandys, Ladies, Anne, iii, 376 Kath., iii, 298, 391 : Letitia, iii, 376 ; Lds., ii, 330; iii, 23 ; Edwin iii, 376 ; Sam., iii, 376 ; Will. ii, 340 ; iii, 298,39 I

Sandys, Sir Edwin, iii, 37r, 391 Eliz., iii, 37r; Hen., iii, 37r Margery, ii, 340; iii, 371 ; Mary iii, 39r ; Miles, iii, $\mathbf{1} 5 \mathrm{r}, 37^{\mathrm{r}}, 39 \mathrm{I}$; Walt., iii, 371 ; Sir Will., iii, $37 \mathrm{I}$; Will., iii, $37 \mathrm{I}$; fam., iii, 324 Sandy Warren (Sandy), ii, I46, 197 $242,244,246$

Sanes, Cecil de, iii, $37^{2}$

Sanford, Rob. de, iii, 66 ; Rog. de, iii, 452

Sankey, Thos., iii, 432

Santingfield Hosp. (Wissant), i $400 ; \mathrm{ii}, 358$

Sanvill (Saunvill), Gilb. de, iii, 285 ; Osmund de, iii, 398; Osmunda de, iii, 285 ; Phil. de, i, 37 In iii, $285,286,288,395,398$; sec also Savile

Sapte, F., ii, 265

Sarnebroc, see Sharnbrook

Sarnes, man. (Ravensden), see Mossbury, man.

Saunders (Sanders), Abra., ii, 385 ; Edw., iii, I55; Ellen, iii, 344 422, 423: Isabel, iii, 423: Jane, ii, 385 ; Joan, iii, 423 : Sir John iii, 130, 310: John, iii, 3 I0, 45 r Marg., iii, 4II ; Mary, iii, I93 Rich., iii, 310, 409, 4 II, 423 Thos., iii, 193, 344, 422, 456 Val., iii, I29, I30: Will., iii, 344 422,423

Saunderson, John, ii, 252 ; iii, 209 Judith, ii, 342 ; Laur., ii, 252 see also Sanderson

Saunderson, H. G:, \& Co., ii, 127 


\section{INDEX}

Saunderton, Bern., iii, 3ro; Will., iii, 310, 313

Saundeye, see Sandy

Saunvill, see Sanvill and Savile

Saurez (Sawrey), Ant., iii, I 51, 391

Savage (Sanvage), Alice, iii, 423 ; Sir Arth., iii, 236; John le, iii, 211 ; Ralph, iii, 423; Rob., iii, 85,423 ; Thos., iii, 236 ; fam., iii, 2 I0, 211

Savile, Lady Eliz., iii, 427

Sawbridge, alderman, ii, 64 ; iii, 20

Sawell (Houghton Regis), see Sewell

Sawrey, see Saurez

Sawtrey Abbey, ii, 228

Sawyer, John, iii, 119

Saxons, i, 188; ii, 18, 73 ; iii, 350 see also Anglo-Saxon

Saye and Sele, Lady, iii, I 79 ; Rich. Fiennes, Ld., ii, 162

Scaccario, Rog. de, iii, $3^{8} 3$

Scale-mosses and liverworts (Hepaticae), i, 58

Scanden, Edm., iii, 129

Scarborough, Eliz., iii, 286

Scargill, fam., iii, 237

Schepehoo, man. (Upper Gravenhurst), ii, 334, 335

Schepehoobrade (Upper Gravenhurst), ii, 334

Schools, ii, I49; farm, ii, I42. lace, ii, I 24 ; plaiting, ii, 120

Schutz, John, ii, 262, $265 n$; Mary, ii, 204, 262 ; Mrs., ii, 207

Sclater, see Slater

Scortegrave, see Shortgrave

Scoteny, Beatrice de, i, 35

Scott (Scot), Ada (le), ii, 210,237

Eliz., ii, 252; iii, I98; Isabel, ii, 237 ; iii, 299 ; John le, iii, 297 299 ; see also Huntingdon, earl of Marg., ii, 237, 238 ; iii, 297 ; Rob., i, 403; ii, 25I-3; Rev. R. C. F., ii, 265; Sam., iii, 198; Thos., ii., 352 n

Scott's Close (Croldington), iii, 109

Scrivener, - , iii, 439

Scroggs (Skroges), Alex., iii, 203, $218 n$; Joan, iii, 216; Oliver, i $335^{7} n$; ii, 40; iii, 203, 216; Rich., i, $335 n$; Will., iii, 246

Scroop's Farm (Biggleswade), ii, 209

Scrope, Hen., Ld., ii, 385

Scrope (Scroop), Hen. le, ii, 2 I I 235 ; John, ii, 384 ; Marg. le, ii, 235, 385 ; Rich., ii, $211,235,385$. iii, 260 ; Rog. le, ii, 385 ; Col., ii, 53

Scudamore, Rich., ii, 356

Seabroke, G., iii, 432

Seayres, Lucy, iii, 415

Sebon, Rog. of, ii, 296 ,

Seckford (Sekford), Hen., iii, 20I : John de, ii, 89

Sedbergh, chant. sch. at, ii, I 57

Seddington (Sandy), ii, 242

Sedgwick, Steph., iii, 405; Will., iii, 405

Segenhoe (Segenehou), man. (Ridgmont), i, 297, 377 ; ii, $76,81,83$, 84,87 ; iii, 267, 320-2; ch., i, 3 I 5 , 37 I $n$; iii, $320-3$; tithes, 1 , 376 ; vicarage, $i, 316 n$

Segrave, Marg., Lady, ii, 339 ; Lds., iii, 12 n, I 33 ; John, ii, 339

Segrave, Christina de, ii, 353 : Eliz., ii, 339; Sir Hugh de, iii, 299; Ida de, iii, 229; Steph. de, iii, 229

Segresdone, iii, I 23

Seiher, see Saher

Sekford, see Seckford

Sellator, Lambert, iii, I I

Seller, Will., ii, I 57
Senhouse (Senhous), Rich., iii, 22, 23 ; Thos. de, ii, 220

Senior, E. N., ii, I97

Senliz (Senlis), Sim. de, i $315 n$; ii, 239

Sergeant, Walt. le, iii, 73

Serle, John, iii, 137 ; Rich., iii, I 37 Serviens, Phil., iii, iा 3

Settle, Elkanah, iii, 355 ; Josias, iii, 355

Sewell (Houghton Regis), iii, 336 389 ; chant., i, $329 n$; ii, I 50, I 5 I iii, 394 ; man., iii, 391 , 392 ; pre hist. rem., i, 169; well, ii, 6, 7

Sewell (Sewelle), Ad. de, iii, 391 ; Hen., iii, 391, 433; Joan de, iii, 433 ; Sir John, iii, 393 ; John de, iii, 390,391 ; Rev. J. E., ii, I 56 ; Matilda, iii, $391 n$; Nich. de, iii, 391 ; Walt. de, iii, $391 n$; Dr. ii, $162 n$

Sewick End (Wilden), iii, 223, 225

Sewster, Will., ii, 282

Sexton's Manor (Wilden), iii, 224 . 225

Seylade, iii, 76

Seymour, Thos., Ld., ii, 270 )

Seymour, Sir Edw., iii, I58; Jane, iii, I I I ; sce also St. Maur

Shaa, Sir Edm., see Shaw

Shakerstone (Shackerstone) (Leics.) ch., i, $3^{88}, 3^{89}$; iii, 65

Shafto, - ii, 192

Shappenho, see Sharpenhoe

Sharnbrook, i, $349,380,394$; ii, 66 , 78,8 I, I I 5,326 ; iii, $34,59,88.94$, I05 $n, 346$; adv., i, 317 ; iii, 94 agric., ii, 83,134 ; chant., iii, 94 char., iii, 94 ; ch., i, 3 I $5 n$; ii, 92 ; iii, 88,92 ; fish-stew, ii, 76 ; lace ind., iii, 89 ; mans., iii, 89 ; mills, iii, 89, 9I ; Nonconf., iii, 89: place-names, iii, 89 ; sch., ii, I 84 ; vicarage, iii, 88,94

Sharnbrook, Hen. de, iii, 52, 9 I

Sharnbrook Grange, iii, 88

Sharnbrook House, iii, 88

Sharnbrook Meadow, iii, 59

Sharnbrook Summit (Wymington) iii, 117

Sharp (Sharpe), Hen., ii, 333 : iii 56 ; Rev. Wilfred, iii, I 88

Sharpenhoe, i, 297, 306, 308, 313 , $345,381-3$; chap., $i, 318 n, 326 n$; ii, $3^{84}$; Chantry Farm, ii, 313 , 384 ; man., ii, 89,382 ; man.house, ii, 384 ; moat, ii, 384

Sharpenhoo, see Sharpenhoe

Sharpin, Rev. F. L., ii, 200

Shathewell, Isabel, iii, 453 ; John. iii, 453

Shaw (Shaa), Sir Edm., ii, I 59

Sheeplane (Potsgrove), iii, 421

Sheeprack Wood (Knotting), iii, I 39

Sheepwalk Hill (Toddington), i, I 85 Sheepwalk Spinney (Turvey), iii, I IO

Sheerhatch Wood (Willington), iii, 264

Sheffield (Sheffeld), Edw., ii, 373 ;

Eliz., ii, 252 ; Marg., ii, 252, 253 ; Thos., ii, 252

Shefford, i, 173, I 74, 187, I89; ii, $1,5,14,44,46,113,265,267 n$, $268,270,271,276,280,286,288$, $294,300,320,333$; iii, $75 n, 313$, 326 ; char., ii, 270 ; ch., ii, 269 , 270,271 ; deanery of, i, 347 ; mkt. ii, 88,268 ; iii, 21 ; Nonconf. ii, 271 ; Rom. Cath., ii, 270; Rom. rem. at, ii, 2 , II-I3, 266 ; Rom. road, ii, 4 ; sch., ii, 184 ; vicarage, ii, $27^{\circ}$

Shefford, Sir Geo., i, 402
Shefford Hardwick (Southill), ii, I1 3 ; iii, 227, 256 ; man., iii, 259

Sheldon, Cuth., iii, 286 ; Prudence, ii, 204 ; Rich., ii, 204 ; fam., iii, 286

Sheldwick, Will. de, i, 362, 365

Shele, Thos., iii, 349

Shelley (Shellelond), man. (Wrest lingworth), ii, 256

Shelton, i, 384 ; ii, 71, I I 5, 324 ; iii $89,123,136,16 \mathrm{I}-5,193,302,3$ I $_{3}$, 332,333 ; adv., iii, 164 ; char., iii 165 ; ch., iii, 163,165 ; mans., i 384 ; iii, 162, 164; ship-money, iii, I62; tithe rent, ii, I06

Shelton, Lower (Marston Moretaine), iii, 308, 313 ; man., iii, 3 I I

Shelton, Upper, hamlet (Marston Moretaine), iii, 308, 333

Shelton, Upper, man. (Wootton), see Wootton Shelton, man.

Shelton Grene (Wootton), iij, 329

Shelton Hall (Shelton), iii, 160

Shelbethorpe, Rob. de, iii, 125

Shelvestrode, Joan de, iii, 252 ; Walt. de, iii, 252

Shengay Preceptory (Cambs), sec Shingay Preceptory

Shenkwyn, Thos., i, 399

Shenley Hill (Leighton Buzzard), iii, 400, 415

Shepherd (Sheparde, Sheppard), John, iii, 429 ; Philippa, iii, 429 ; Rich., iii, 444; Rob., iii, 4 II ; Sarah, iii, 444; Thos., iii, 316 , 385 ; Will., iii, $3^{8} 3,3_{4}$; -, i, $342 n ;$ iii, 18,30

Shepherd's Bush (Dunstable), Rom. rem., ii, 6

Sherard, Geo., iii, 386, $387 n$ : Marg., iii, 386 ; Rich., iii, 386 . 387 ; Thos., iii, 386

Sherborne (Sherbourne), Ad. of, i, 381 ; Rob., iii, 301 ; Sophia, iii, 301 ; Sir WVill., i, $368 n, 369$

Sheriff, Rich., iii, 446

Sherley, Thos., see Shirley

Sherwin, Thos., ii, 299

Shillington, i, $276,324,327$; ii, 21, 25, 77, 78, 90, I I 3, 260, 26r, $288,293-9,304,308,333,344$; adv., i, 324 ; ii, 299 ; chant., ii, 299; char., ii, 299 ; ch., i, 3 I 3 ; ii, $78,293,297-9,335$; coprolite, ii, II 7 ; copyhold, ii, 90 ; farms, ii, 82,83 ; labourers' wages, ii, 80 ; mans., i, 312 ; ii, 79, 294-6, 374 ; iii, 238 ; mills, ii, 293, 297 park, ii, 297 ; Nonconf., ii, 299 place-names ii, 294 : poor-house, ii, I04; Rom. rem., ii, 13, 294 ; scb., ii, I 84,293

Shillington Bury, man. (Shillington), i, 307 ; ii, 295

Shingay (Shengay), preceptory of (Cambs), ii, 277; iii, 23, 213 , $324,330,332,376,378,420,437$

Shipley, Ellis, iii, I46

Shipman, Eliz. (Lucy), ii, 204

Ship-money, ii, 4I; iii, 35, I62 I 80, 197, 227, 267

Shire Horse Society, ii, 137, 140

Shirley (Sherley), John, ii, 316; Thos., iii, 167

Shitlington, see Shillington

Shoe-making ind., iii, 157

Shone, Will., ii, 318

Shortewood (Bolnhurst), iii, I 26

Shortgrave, man. (Studham), ii, $31,145,376,430,449 n, 456$

Shotbolt, Thos., iii, 432

Shotesbrook (Shottesbroke, Shottisbrook), Edith, Lady, iii, 136 ; John de, iii, I36; Sir Rob., iii, 107, I $36,137,317$ 


\section{A HISTORY OF BEDFORDSHIRE}

Shouldham Priory, i, 39r

Shrewsbury, earls of, ii, 203,223 , 227, 261 ; Fran., ii, 340 ; Gilb., ii, 261: John, ii, 223

Shropshire, wool, price of, ii, 9 I

Shutlyngdene, see Shillington

Shuttleworth, Caroline, iii, 255 ;

Col. Frank, ii, 197: iii, 202, 205 , $209,240,251,253-5,257,264$; Jos., iii, 205, 253, 257

Shyfforth, see Shefford

Shyngay, see Shingay

Shytlington, see Shillington

Sibelthorpe, Edw., iii, 18

Sibley, Anne, iii, 429; Edm., iii, 429: Edw., iii, 429; Eliz., iii, 429: John, iii, 429; R. G., ii, 374 ; Thos., iii, 346, 429; Will. iii, 346 ; fam., iii, 194,348

Sickling, Thos., iii, 425

Sidegate (Suff.), iii, 229

Sidney (Sydney), Algernon, ii, 57 Mary, iii, I $77 n, 290$; Sir Phil. iii, 290; Sir Will., iii, $177 n$

Sidney Sussex Coll. (Cambridge), ii, 180 ; iii, $179,296,328,332$

Silchester (Toddington), Rom. rem., ii, $2,13,15$

Sill, Simeon, ii, 212

Silisoe, i, 174, 297, 318 ; ii, 36,146 , $289 n, 325-33,335,336, \quad 382$ chant., ii, $326,330,331,332$ chaps., i, 315n, 318, $325 n$; gold coin, ii, 326 ; man., ii, 329 , 330 iii, 292 ; mit. and fairs, ii, 88 330 ; pop., ii, II 4 ; sch., ii, I 84

Silsoe, John of, $\mathbf{i i}, 336$

Silver End (Haynes), ii, $33^{8}$

Silverton Grange, see Goldington Grange

Silvester, ii, 78

Simley, - , ii, 44

Simmons, F., iii, 351 ; Thos., ii, 120

Simon, anchorite, i, 350 ; the hermit, i, 350

Simons, Hen., ii, 318

Simpson (Bucks), iii, 349

Simpson, F., ii, I98; Rich., iii, I $32 n$

Sinclair, Geo., ii, 135

Sindry, John, iii, 21

Sismey, John, iii, 155

Sitegate (Sharnbrook), iii, 91

Siward, ii, 237

Skeffington, Ant., iii, $162 n$; Geofi. de, iii, I68; John, iii, I63; Thos. iii, 163; Sir Will., iii, 163; Will. iii, 163 ; fam., iii, 162

Skegness, Bridg. F. A., ii, 295 ;

Frances, ii, 295

Skinner, Tom, ii, 188

Skippon, -, ii, 46

Skipworth, Eliz., Lady, iii, 435 Greg., iii, 344 ; Sir Will., iii, 435

Skroges, see Scroggs

Slade House, The (Hinwick), iii, $8 \mathrm{r}$

Slade Plantation (Podington), iii 80

Slater (Sclater), Chris., i, $338 n$; iii, 4I4; Rob., ii, II8; Will., ii, 312; -, ii, I19; fam., iii, 4II

Slayny, Marg., Lady, iii, 250

Sleaford (Lincs), A.S. rem., i, 182, I 84

Slingsby, Abija, iii, 398 ; Hezekiah iii, 385: Kath., iii, 385 ; Mark, iii, 399 ; Rebecca, iii, 385 ; Rob., iii, 398

Slip End (Caddington), ii, 314

Slipp (Luton), ii, 36I

Slow, Rog., ii, 204

Slye, John, iii, 18

Smalman, Jas., iii, 159

Smart, Arth., ii, 375; Gerard, iii, I07
Smartwick (Welden), iii, 223

Smeaton, -, iii, 235

Smith, Ábra., iii, 226; Alicc, iii 429: Anne, iii, 177 ; Rev. A., iii, 429: Barbara, iii, I77: Benett, ii, $33^{6}$; Charlotte O., iii, $3^{8}$, 388; Chris., iii, 395 ; Cyrus, ii, I $85 n$; Rev. C. H., iii, I79; Diana, iii, 6o; Dorothy, iii, 205 . Edm., iii, 177; Rev. Edw., iii, 60; Rev. Edw. O., iii, 388 ; Eliz., iii, 429 ; E., ii, 174 ; Frances, iii, 256, 261; Geoff., iii, 49; Geo. ii, 22I, 408; Hen., ii, 24I; iii, 25I; Jas., ii, 24I ; John, iii, 392, 429, 432: Magdalen, ii, 233 : Martha, iii, 432; Mary, ii, I62; iii, 432; Matth., iii, I25; Nich., i, 387 ; Rich., iii, 226 ; Roach, i, I 76 ; Rob., ii, 24 I iii, I 56 ; Sim. ii, 336 ; Thos., ii, 316 ; iii, 246 247 ; Sir Will., iii, 255 ; Will., i, 335 ; ii, 162, 335 ; iii, 201,346 , $348,349,367,432$; Worthington G., i, 269, 270; ii, $3 n$; Mrs. Chernock, iii, $386,387,388,425$; ii, 165; see also Smyth and Smythe

Smithies, Rev. H. Y., ii, 133

Smyth, Alice, iii, 393; Edw., ii 256 ; Geo. E., ii, 256; Jas., ii, 256; John (de), iii, 137, 255: Rev. Laur., iii, $255 n$; Rich., i, 353: iii, 225; Rob., iii, 293 : Susanna, iii, 255 ; Thos., iii, 456 ; Will., ii, 150; iii, 293; see also Smith and Smythe

Smythe, Geo., ii, 40, 382 ; iii, 106 ; Hugh, ii, 382,383 ; Jas., ii, 382 383,385 ; Jasper, ii, 263 ; John, ii, $263,305,382$; Marg., ii, 385 Rev. Rog., ii, 265 ; Thos., iii, I06; Will., ii, 162; iii, 106, 394 Mrs., ii, 382; see also Smith and Smyth

Snagge, Agnes, iii, 13, 313; Anne (Anna), iii, I3, 298, 312 ; Beatrice, iii, I3: Chas., iii, 298: Edw., iii, 306, 3c8, 309; Eliz., iii, 298, 306 309; Geo., ii, 363; Mary, ii, 363 Ralph, iii, 298; Sir Thos., iii, I3, 240, 298, 309, 321 ; Thos., ii, 296 . iii, II, I2, I3, I4, 298, 299, 306, 307, 309, 31 2, 313; 一, iii, 2 I

Snell, Will., i, 402

Snetteshall Priory, iii, 184

Snitch, John, ii, 24 I

Snowe (Snow), Alice, iii, 301 ; Dan. ii, 270, 27I; Edw., ii, 271, 275 . 276, 344; iii, 301 : Eliz., ii, 271 276,342 ; iii, 301 ; Emma, ii, 27 I Joan, ii, 363 ; Lawr., ii, 336,378 ; Rebecca, iii, r65, 30r ; Rich., ii, 270, 271, 275, 276, 342 ; Sarah iii, 3or; Thos., ii, 336; Will., ii, 334,378 ; farn., iii, 297

Soame, Judith, ii, 231, 233; Sir

Steph., ii, 23I, 233

Social and economic hist., ii, 73-I I 6

Socon, see Eaton Socon

Soham (Cambs.) i, I89

Sok, Godf. le, iii, 196: John le, iii I96; Rog. le, iii, rg6; Sim. le, iii, r96; Will. le, iii, 196

Sokes Manor (Laurence Placc) (Eaton Socon), iii, 196

Soleby, Hen. de, iii, I62, 164 ; Joan de, iii, I62; Rob. de, iii, I 62 Will. de, iii, 162

Solemn Thrift (Bromham), see Salem Thrift

Someries, man. (Luton), ii, 350, $352 n, 358,372$

Someries Castle (Luton), i, 305 ; ii, $34^{8}, 364$
Somers, Rich., ii, 3 I 5

Somerset, Marg., dchss. of, iii, 4I, 137, I8I; dks. of, ii, 37, 316: iii, 137; Edw., iii, I58; Hen., iii, I73: John, iii, 4 I

Somerset, Sir Chas., iii, I 73 ; Geo., iii, r73: Hen., iii, r73: Thos., iii, I4

Somersham, Agnes de, ii, 248 ; Alex. de, ii, 248; John de, ii, 248; Marg. de, ii, 248

Somery, Agnes de, ii, 364 ; Joan de, ii, 364; John de, ii, 364 ; Marg. de, ii, 364 ; Rog. de, ii, 364

Sondeye, see Sandy

Sondon (Sonedone), see Sundon

Son of Athelwine, Ailward, ii, 2 I

Son of Alan, Will., ii, 8

Son of Alchetil, Will., ii, 78

Son of Alex., Jordan, iii, 431 ; Walt.,

iii, I73

Son of Ascelin, Rob., ii, 296

Son of Azon, Will., iii, I59

Son of Azor, Hen., iii, 57, 58

Son of Baldwin, John, iii, 2 I6

Son of Benedict, Cok, iii, 2

Son of Bernard, Thos., iii, 248

Son of Brun, Mossy, i, 321 ; iii, 363

Son of Bryan, Rob., iii, 418 n, 419, 42

Son of Drew, Ad., iii, 98

Son of Edwin, Lewin, ii, 314

Son of Erfast, Nigel, ii, 281, 285

Son of Ernest, Arnold, iii, 377 Felicia, w. of Arnold, iii, 377

Son of Ernis, Rob., iii, I 44

Son of Eudo, Will., iii, 2 I I

Son of Flemengo, Leo, iii, 363

Son of Frane, Oswulf, iii, 426

Son of Fulcher, Amabel, d. of Will. iii, 285 ; Osmunda, w. of Will., iii, 285 ; Will., iii, 285

Son of Gerard, Warren, iii, 343

Son of Gerold, Hen., ii, 267, 268 : Warine, ii, 267, 268

Son of Gilbert, Rich., see Clare, Rich. de

Son of Godric, Eadnoth, iii, I 50

Son of Godwin, Gyrth, ii, 20 ; Harrold, ii, 20 ; Leofwine, ii, 20 ; Swegen, ii, 20 ; Tostig, ii, 20

Son of Gozelin, Hugh, iii, 408

Son of Gregory, Walt., iii, 424

Son of Henry, Guy, iii, 302

Son of Hordfast, Nigel, ii, 263

Son of Hubert, Eudo, ii, 248, 253 jii, 245, 247 ; see also Dapifer

Son of Hugh, Barth., ii, 326 ; Geoff., iii, 314 ; Hen., ii, 244

Son of Ilger, Rannulf, iii, 78

Son of Ivo, Herb., iii, 49, 50, 97

Son of Jocelyn, Hugh, i, 37 I $n$

Son of John, Ad., iii, 427 ; Rob., ii,

Son of Jordan, Rich., iii, 155 ; Rog., iii, 155 ; Thos., iii, 166

Son of Juliana, Hen., ii, 85

Son of Margaret, Rob., iii, $3^{8}$

Son of Mary, Ad., iii, 58

Son of Nigel, Rob., iii, 78

Son of Olympeas, Rob., ii, 275

Son of Osmund, Levric, iii, 433

Son of Peter, Hamon, iii, 106

Son of Pictavus, Benedict, iii, 2 : Jacob, iii, 2

Son of Rainald, Will., iii, 324

Son of Raineward, Will., iji, 2 Ig

Son of Ralph, Rob., ii, 44 ; iii, 181

Son of Richard, Gilb., iii, 428 Osbern, iii, 159 ; Paschasia, w. of Gilbert, iii, 428; Ralph, i, $325 n$; Will., iii, 324, 325

Son of Robert, Sim., ii, 286

Son of Roger, Hen., ii, $35 \mathrm{r}$; Ralph, iii, II3 


\section{INDEX}

Son of Roland, Abel, i, 37r $n$; iii, 29

Son of Rozelin, Rob., iii, 90

Son of Salaman (Salomon), Gilb. ii, 288 ; iii, 59

Son of Sampson, Rob., ii, 379

Son of Sewale, Thos., iii, 36

Son of Simon, Rich., ii, 360 ; Rob. ii, 360 ; Walt., iii, 436

Son of Siward, Alan, ii, $8 \mathrm{I}$ Waltheof, iii, I68

Son of Teodric, Rog., iii, 206

Son of Turkill, Rob., ii, 78

Son of Walter, Osbern, ii, 207, 208

Rob., iii, 396; Walt., ii, 3I5

Son of Warin, Agnes, w. of Walt., ii, $234 n$; Walt., ii, $234 n$

Son of William, Gilb., ij, 60 ; Hen. ii, 244 ; Rich., ii, 225

Son of Wymark, Ralph, iii, 74

Sonyngdon, see Sundon

Sopwell Priory (Herts), i, 35 I $n$ : ii, 324 ; iii, 237

Sorthegrave, see Shortgrave

Soulbury (Bucks), i, 162; iii, 368, 404,415 ; ch., i, 370 ; rectory, i, 370

Soulbury, Hugh of, i, 370

Souldrop (Soulthorp), i, $327 n, 394$; ii, 90 , II 5 ; iil, $34,94-5$; adv., iii, 95; char., iii, 95; ch., i, $3 \mathbf{1} 4$ n, 394; iii, 95 ; lace ind., iii, 94 ; man., iii, 95; place-names, iii, 95 ; schs., ii, I 84 ; iii, I39

Souldrop, Steph. of, i, $314 n$; Will. of, i, $382 n$; ii, 244

Souldrop Would, iii, 94

Soulthorp, see Souldrop

Southampton, Thos. Wriothesley, earl of, ii, 57 n

South Brook (Wilden), iii, 223

Southill, i, 364, 365, 380, 393 ; ii, $44,55 n, 60,61,66,116,179,197$, 266,338 ; iii, $69, \mathbf{1} 75, \mathbf{I} 76,246$, 256-26I, 28I, 283; adv., iii, 26I ; agric., ii, I 34 ; char., iii, 261 ; ch., i, 315n, $378 n$; iii, 260 ; man. iii, 257; mill, ii, 278; placenames, iii, 257; priest's stipend, i, 34I ; iii, 261 ; schs., ii, 184; vicarage, i, $3 \mathrm{I} 7$; warren, iii, 260 Southill Park, ii, I47, 190; iii, 234 , $235,256,258,261$

Southill with Rowney, iii, 256

South Mills, man. (B]unham), iii, 228,231

Southoe, man. (Hunts), iii, I72

Southouse, Edw., ii, 36I ; Hen., iii, 2 II; Sarah, iii, 2 II

Southwater Mead, ii, 266

Southwell, Thos., iii, 82, 419

Southyevell, see Southill

Sowerby, John, ii, 36r ; Col., ii, 359 ; Maj., ii, I96; -, ii, 359, 361

Spark, Sir John, i, 326

Sparrow, Rob., iii, 20

Spech (Spec), hon. of, iii, 219

Spech (Spec, Espec), Albreda, iii, 176, 257; Aubrey, iii, 219: Hadwisa, ii, 230 ; iii, 252; Walt., i, $361,362 n, 364$; ii, 230, 233; iii, $36 n, 175,176,243,252,253$, 255, 257, 259: Will., ii, I 43,230 , $383 n$; iii, $36,38,85,120,134$, $219,220,22 \mathrm{I}, 248,252,257^{-8}$, $324,386,439$; fam. iii, 207

Speedwell Belt (Potsgrove), iii, 42 I Spelman, Sir Hen., i, $343 n$

Spence, John, iii, I94; Rob., iii, 194

Spencer, baron, ii, 243; earls, ii, I 80, 220, 222; iii, I04, 3IO : John, iii, 306; Chas., Ld., ii, 220 ; Rob., Ld., ii, 222
Spencer, Alice, iii, 102 ; Arnold, iii, 314 ; Eliz., iii, 208; Frances L. iii, 301: Hen., see Sunderland, earl of ; Sir John, ii, 243; iii, 102 208: John, i, 393: ii, 62, 163 $220,232,245$; iii, $\mathrm{IO}^{2}, \mathrm{I}_{2}, 240$ 306, 310 ; John W., see Brownlow, earl:Mary, ii, 232, 243: Nich. iii, 29, 184, 240; Rev. Oliph L., iii, 30I : Rob., ii, 43, 220, 222 , 243 ; iii, 182,240 ; Thos., ii, 245 ; iii, 240; Will., ii, 220, 243 ; $\mathrm{iii}_{\text {, }}$ I $82,240,242$; fam., iii, 205

Spendlove, John, ii, $360 n$

Spensley, Howard, iii, 45I ; Mrs. iii, 453

Spicer, John, iii, I66; Rich., iii, I 54 ; Thos., ii, 40 ; iii, 332

Spiders (Arachnida), i, 88-90

Spigurnel, Alice, i, 360: Sir Hen. iii, 428 ; Hen., ii, 276,286 ; iii I29, I 30, 429; Joan, iii, 429 ; Lucy, iii, 429; Phil., iii, I29n: Sarah, iii, I30; Sir Thos., ii, 276 280 ; Thos., ii, $286 n$; iii, I 29 , 428 ; Will., ii, 286-7; iii, 429;

Spiller, Sir Hen., iii, II 3

Spinneyhole (Maulden), iii, 316

Spittle Close (Dunstable), iii, 364

Splashyard Wood, see Palace Yard

Spokes, Rev. J. H., ii, zo 8

Sports, ancient and modern, ii $187-200$

Springfield House (Kempston), iii, 297

Springs, chalybeate and medicinal i, 39 ; iii, $44,59,69,96,284$ petrifying, ii, 308 ; iii, 82

Spurling (Spurlyng), Anne, ii, 224, 232; John, ii, 224, 232

Spynk, John, iii, 52

Squire, Gaius, iii, I91, 198 ; John ii, 204, 205, 320; Mary, iii, I9I Rob., ii, 204, 205; Rowland (Roland), iii, 191, 196; Miss, iii, 193

Stacheden, see Stagsden

Stachesdene, see Stagsden

Stacy, Agnes, iii, 247 Edw., iii, 244, 248; John, iii, 247 ; Thos., iii, 247,248 ; Will., iii, 28

Stacy's Wood (Northill), iii, 245

Stafford, man. (Kempston), iii, 302

Stafford, Anne, ctss. of, iii, 17 I earls of, Edm., iii, 156, I 7 I Ralph, iii, 36; Thos., iii, 36 Lady Marg., iii, 36; Will Howard, visct., iii, 192, 196

Stafford, Anne, iii, I4; Ant., iii, 196: Bridg., iii, 196: Edw., see Buckingham, dk. of ; Eliz., iii, 195, 196, 283, 298 ; Sir Humph. iii, I4; Humph., iii, 195, 196 Sir Hen., iii, 298; Jane, iii, 302 John, iii, I96; Kath., iii, I95. Marg., Lady, iii, 298: Mary, iii, r96: Nigel de, i, 353; Thos., iii, 277, 302: Walt, dii, 196: Sir Will., iii, 195, 298; Will., iii, 196, 302 ; fam., iii, 76

Stagsden (Stagesden, Stachesdene) ii, II 5 ; iii, 32, 34, 96-100; adv. iii, roo; ch., i, 3I $5 n, 380$; iii, 98 ; char., iii, 100 ; farms, iii, 96 lace ind., iii, 96: man., iii, 96 mills, iii, 96; park, ii, 143 sch., ii, I 84 ; vicarage, $i, 316 n$

Stagsden, John of, i, 352; fam., iii, 199

Staines (Stands), Capt. John, iii, 12 ; see also Stane

Staismore fam., see Staysmore

Stamford (Lincs), ii, $276 n, 290$, 299
Stamford, Rich. of, ii, I7 8

Stampe, John, iii, 277

Stanbridge, ii, 375 ; iii, 368,399 $400,402,407,415,417,447$ chant., iii, 4I5; chap., i, 324 , 34I ; iii, 4 I 4 ; ch., iii, 4 I2 ; man. i, 381 I ; iii, 405: Nonconf., iii, 4 I 5 ; pop., ii, II 4 ; schs., ii, 184 . vicarage, iii, $4^{\mathrm{I}} 4$

Stanbridge, half-hund., iii, 336

Stanbridge, Hugh of (de), iii, 347 $44^{2}$ : Isabel of, iii, $347^{\circ}$; Joan of i, 360 ; Sim. of (de), iii, 347,442

Stanbridge Ford (Totternhoe), iii, 400,447

Stanbrig (Stanbrugge), see Stanbridge

Standon (Beds), see Stondon, Upper

Standon (Herts), i, 3I2

Standon, Ralph de, ii, 296 ; Walt. de, $i, 352$

Stands, see Staines and Stane

Stane (Stanes), Christina de la, ii, 321 ; Eliz. de la, ii, 321 ; Margery de la, ii, 32 I ; Pet. de la, ii, 32 I Will. de, ii, 350; see also Staines

Stanford (Southill), i, 365, 393; iii, $227,256,258$; fishery, iii, 259 ; man., iii, 258 ; mills, iii, 256,259 ; Rom. rem., ii, I, I3

Stanford, Ld, ii, 44

Stanford, Eliz., ii, 289 ; Geoff. of, i, 365: Joan, ii, 289: John, ii, 289; iii, 97 ; Mary, ii, 289; Will., ii, 29

Stanfordbury Farm (Southill), iii, 257,258 ; Rom. vault, ii, I3

Stanfordbury Manor (Southill), iii, 258,259

Stanhope, Edw., iii, 236, 4 I8 ; Mich. iii, 236, 4I8; Phil., see Chesterfield, earl of

Stanley, Ld., iii, 230 ; Thos. Ld., iii, $140, \mathrm{I}_{72}$

Stanley, Edw., iii, ro2, I19; Eliz. Lady, iii, 405; Ferdinand, iii, I19; Sir Geo., iii, I19; Geo., see Strange, Ld.; Hen., see Derby, earl of; Sir John, iii, 145; Marg. iii, 283; Thos, see Derby, ear of; Sir Will., iii, 405 ; Will, iii, IO2

Stanton, see Staunton

Stanwick (Northants), i, 312; ii $7 \mathrm{I}, 72 ; \mathrm{iii}, 123$

Stap, Dorothy, ii, 373 ; Roland, ii, 373

Stapleford Mill (Luton), ii, 366

Stapleton, Sir Phil., ii, 45 n

Staple Wood (Eaton Socon), iii, 189

Staploe (Nether Staploe) (Eaton Socon), i, 297 ; iii, $190,192 n_{1}$ 193, 197,198

Staploe, Upper (Over), (Eaton Socon), iii, 190, 193, 201

Starcher, iii, 402

Stare, Edw., ini, 405 ; Mary, iii, 405 Will., iii, 405

Staresmore, see Staysmore

Starkey, - , ii, I66

Starling (Starlyng), Sir Sam., ii, 359 ; Will., ii, $360 n$

Staughton, Little, ii, 7I, 86, II 5 , I 33 ; iii, I23, I36, I $46 n$, I 53 I65-8; adv., iii, I67 ; char., iii I68: ch., i, $315 n, 349,394 n$ : iii, $146 n, 166$; man., iii, 165 : Nonconf., iii, r65; place-names, iii, 165 ; sch., ii, I 83

Staunton (Stanton), Edw., iii, $46 \mathrm{r}$ Eliz., iii, 46I ; Sir Fran., ii, 40 iii, 4I 8, 46r, 462 ; Fran., iii, 4I 8 John de, iii, 376 ; Rob., ii, 44 iii, 259; Stavely, iii, 46I ; Will. iii, 4 I 8 ; 一, iii, 32 I 


\section{A HISTORY OF BEDFORDSHIRE}

Staysmore, man. (Carlton), see Carlton $\mathrm{Hal}$

Staysmore (Staresmore), John, iii, 5I, 384 ; Will., iii, 5I, $3^{84}$

Stead, Geo., iii, 313

Steingrave (Steyngreve), Ida, iii, $45,235,295,330$; Isabel, iii, I 4 ; John de, iii, 14, 235, 295, 424: Mabel de, iii, $33^{\circ}$

Steph, Will., iii, 50

Stephen, king, i, $372 n$; ii, 24, 25 ; iii, 9,352

Stephen, priest, iii, 286

Stephen, Maj. J. Y., iii, 428; see also Steven

Stephens, Agnes, i, 36I ; Jeremy, i, 338, 343; Thos., ii, 268 ; see also Steven.

Steppingley (Stepigelai, Stepingle), i, 394 : ii, 115 ; iii, $267,324-5$, 458 ; adv., i, 376 ; iii, 325 ; char., iii, 325 ; ch, i, 315 $n, 321,37$ I $n$, $374 n$; iii, 325 ; 'French Horn' Inn, iii, 324 ; man., i, 364 ; iii, 23 , 324 ; mills, iii, 325 ; Nonconf., iii, 324 ; place-names, iii, 324 ; sch., ii, I 84 ; warren, ii, 136 ; iii, 324

Steppingley (Steppingly, Steppingle), Hen. de, iii, 324 ; John de, iii, 324 ; Maud de, iii, 324 ; Nichola de, iii, 324 ; Rich. of, $i$, 37 I $n$; iii, 324,325 ; Walt., iii, $220 n$; iii, 324,325

Steppingley Park, iii, 325

Steppingley Park Farm, iii, 324

Steppingley Wood, ii, 145 ; iii, 324

Stepynge, Mattl., iii, 240

Sterne, Susan, ii, 329, 330, $37^{8}$ Thos., ii, $329,330,378$

Stethintun, see Stevington

Steven, Hen., ii, 379; see also Stephen

Stevenage (Herts), ii, 4 ; iii, 293

Stevens, -, ii, I18; see also Stephens

Stevenson, Rob., iii, 282 ; W. H., i, $310 n$

Stevington, i, $388 \mu$; ii, 77,91 , 115 : iii, $34,44,100-4$; adv., i, 389; iii, 103,; Black Death in, iii, roo; char., iii, I04; ch., i, 3 I5 $n, 324 n, 3^{88}, 3^{89}$; iii, 102 cross, iii, IOO; fishery, iii, IO2 ; ind., $\mathrm{iii}, 100$; man., ii, 88 ; iii, 74 , 78 , I oo; mill, ii, 89 ; iü, Ia); placenames, iii, I03 $n$; rents, ii, 132 sch., ii, 184 ; vicarage, i, $316 n$

Stevington, Alwold of, iii, I I 4

Stevington Hall, iii, IO2

Stevington Park, ii, I87; iii, 102

Stewart (Steward), Anne, ii, 281 Eliz., iii, 32: Geo., ii, 281; Lt. Col. Jas., ii, 67; Will., ii, 2 10; see also Stuart

Stewkley (Bucks), i, 370, 404

Steyngreve, see Steingrave

Steynton, see Stevington

Stigand, archbp., ii, 210,219 ; iii,

Stilton (Hunts), i, $386 n$; ii, I 20

Stirling, Thos., ii, 318

Stirman, John, iii, 453

Stivecle (Stivecele, Styvecle), Alice, ii, 334 ; Aline de, ii, 334 ; Barnabas, ii, 334: iii, 252 ; Joan, ii, 334; Joscelin de, ii, 333, 334; Nich. de, iii, 194; Walt. de, ii, 334 ; iii, 252; see also Stukeley

Stiventone (Stivetone), see Stevington

Stoakes, see Stokes

Stobart, F. W., iii, 44

Stobell, Anne, iii, 300 ; Will., iii, 300
Stocker (Stokker), Hen., iii, 201 : John, iii, 201; Oliver, iii, 197 Rich., iii, r97: Sir Will., iii, 201 Stock Grove (Bucks), iii, 404

Stocking Croft (Ravensden), iii, 2 10 Stockinge (Shillington), ii, 295 n

Stockinge Close (Kempston), iii, 302 Stocking Pelham (Herts), ii, 214 304

Stockwood Close (Luton), ii, 36 I

Stockwood Park (Luton), ii, I 47, $197,319,348,360,361$; iii, 105 , 177

Stocton (Stokton), John de, iii, I65 Rich. of, ii, I 49 ; fam., iii, 166

Stocton Parva, see Staughton, Little

Stocwell, Urian, iii, 295

Stodden, hund., ii, I1 5 ; iii, 123,180

Stodham, see Studham

Stoke, man., i, 376 ; mill., iii, 9

Stoke, John de, iii, 67

Stoke d'Abernon (Surrey), iii, 78

Stokehammond, man. (Bucks), i 376

Stoke Rochford (Lincs), iii, 145

Stokes (Stoakes, Stokys), John, iii, I2I ; Rob. de, i, 370; iii, 152 : Will. de, iii, 286, 288

Stokesby, man. (Norfolk), i, 360

Stokesholt, ii, 291

Stokker, see Stocker

Stokton, see Stocton

Stokton Parva, see Staugliton, Little

Stokys, see Stokes

Stolfalt, see Stotfold

Stolferth, iii, 383

Stonbury, see Etonbury

Stondon, Lower (Shillington), ii, 260, 293, 294, 296, 304 ; char., ii 287

Stondon, Upper, ii, 113, 260, 275, 288 , 304-5; adv., ii, 305 ; ch. i, $315 n$, ii, 304 ; coins, Brit. and Rom., i, 173, 174; ii, 304; man. ii, 304

Stondon, Will. de, iii, 5 I

Stone (Bucks), Rom. well, ii,

Stone, John, iii, 321 ; Rich., iii, 322 ; Thos., iii, $32 \mathrm{I}$; Will., ii, $40,130,131$; iii, $322,34^{\circ}$; ii, 96,136

Stoneham, Mary, ii, 243 ; Rob., ii, 243

Stoneley Priory (Hunts), iii, $\mathbf{I}_{73}$

Stoneworts (Characeae), i, 59

Stoneylove, Thos., ii, 286

Stonley, Rich., iii, 212

Stony Hill (Limbury), Rom. rem. ii, 8

Stoppesley, see Stopslcy

Stopsley, ii, 306, 308, 348, 349, 350, $357,361,374$ : adv, ii, 374 ch., i, 346 ; ii, 374 ; man., ii, 350 , $352 n, 359,363,365$; Nonconf., ii, 374

Stopsley (Stoppesley), Alex. (de) ii, 365 ; iii, 130 ; Edw., ii, 365 Hugh de, ii, $366 n$; John of, i, 352 ; Matilda de, iii, 130

Stortewayle, Will., i, $4^{\circ 2}$

Story, John, ii, 40; Phil., ii, 227

Stotfold (Stotfeld), i, 379, 380: ii, II $3,206,2 I_{4}, 260,261,300-4$ : adv., ii, 303; agric., ii, 134 char., ii, $184 n, 303$; ch., i, 3 I $5 n$ 392 : ii, 300, 302: Cook's Close, ii, 304 ; ind., ii, 117,120 ; mans., i, 381, 393: ii, 300; iii, I2: mills, is, 302 : Morrells Close, ii, 304: Nonconf., ii, 303: placenames, ii, 300 ; poor-house, ii, I04 : priest's stipend, i, 336 Rom. rem., ii, 14: Rom. rd.,
Stotfold (cont.)

ii, 4, 300; schs., ii, I 84,304 vicarage, $i, 3 \mathbf{r} 6 n$

Stotfold, Joan, iii, 221 ; Walt., iii, 221

Stotfold Brayes, man. (Stotfold), ii, 300,302 ; iii, 44 ; mills, ii, 300 , $301 n$

Stotfold Common, ii, 300

Stotfold Green, ii, 300

Stotfold Newnham, man. (Stotfold), ii, 301

Stotford, Geoff. of, ii, 289 n

Stotham, see Studham

Stotton, ii, 83

Stoughton, Little, see Staughton, Little

Stoughton (Stouton), Joc. of, i $386 n$; Rich. of, i, 386,387 ; Thos., i, 387 ; Walt. de, iii, I37: Will., i, $3^{87}$

Stouton, see Stoughton

Strabolgi, Dav, de, iii, 3, 23r

Strafford, Lady Anne, ii, 277: iii, 441 ; Id., ii, 52 ; Thos., Ld., ii, 277 ; iii, 44 I

Straillei, see Streatley

Strange, Geo. Stanley, Ld., iii, 172

Strange, Marg., iii, I 45 ; Rog. le, iii, 418; Thos., iii, 384 ; Walt. iii, I45: Will., iii, 144, 145, 368 , 394

Strangeways, Sir John, iii, 391

Strangward, Will., iii, I 32

Stratford (Sandy), i, 27 I ; ii, 242

Stratford, Thos. of, ii, 384

Stratford, Langthorne (Essex), ii, 227, 252, 253

Strathum, 'Eliz, iii, I29; Thos. iii, 129

Stratlei, see Streatley

Stratton (Biggleswade), ii, $45 n$, $201,209,24^{8}$; iii, $69,25^{8}, 259$; chap., ii, 2r4; man, ii, 2 ro; water-mill, ii, 2 I 2

Stratton Park (Biggleswade), ii, I90, 209

Straw-cutting industry, ii, 120

Straw-plaiting industry, ii, I07 I08, 109, I 18-22, 2 I 2, 349, 350 iii, $5,35,234,317 n, 345,35$ I, $363,369,390,426,435,439,447$ Streatley, ii, 89, I1 4, 306, 31 3, 324, $345,391,384$; adv., i, 360 ; ii, $3^{8} 3$; char., ii, $3^{84}$; ch., i, 3 I $5 n$; ii, 383 ; man., ii, 346 , $3^{81}$; mill, ii, $3^{81} n$; place-names, ii, 381 ; sch., ii, 184,384 ; vicarage, i, 317

Streatley Ficld (Streatley), ii, 3 I 3 Stredlegh, see Streatley

Streeter, John, ii, 3 I 5

Street Field (Dunstable), Rom. coins, ii, 6

Strickland, Chris., iii, I79

Strigul (Chepstow), prior of, ii, 265

Stringer, Anne, ii, 291 ; Hen., ii, 290 : John, i, 398 ; Rich., ii, 289 . 291 ; Thos., ii, 290 ; iii, 204

Strode, Edw., iii, 332 ; Litton, ii 301 ; Rob., ii, 316 ; Will, ii, 316

Stuart, Lt.-Col. Dugald, ii, 25 I, 252 ; Col. Geo., iii, 106: Hen., ii, $66 n$; Jas., iii, Io6: May, iii, Io6: Lt.-Col. Will,, ii, 70 : Will., ii, $66 n, \quad 184 n, 252$; Col., ii, 69 ; -, ii, 197; fam., ii, 66 ; see also Stewart

Studde, Eliz., iii, 400

Studham, i, 404; ii, 7I, 84, 92 n 114,314 ; iii, $336,337,368$ 426-32, 455 ; adv., i, 314 $n, 376$. iii, 431; char., iii, 432 ; $\mathrm{ch}$. i, 3 II $n, 3$ I5 $n, 318,37$ I $n$; iii, $428 n, 430$; Ch. Farm, iii, 427 ; 


\section{INDEX}

Studham (cont.)

ind., iii, 426; man., i, 376 ; iii, 426: Nonconf., iii, 432; placenames, iii, 426 ; schs., ii, I84 iii, 432 ; vicarage, i, $316 n$; woollen hat petition, iii, 429

Studham (Stodham), Alex. of, i, $371 n$; iii, 427, 428, 431; Alice de, iii, 428 ; Jordan de, iii, 428 ; Lavinia de, iii, 428; Paschasia de, iii, 428: Rob. de, iii, 428, 431 : Sarah de, iii, 428 ; Thos. de, iii, 428 ; Will. de, iii, 428,431

Studham House (Studham), iii, 428

Studley, Thos., iii, 33

Studleys, man. (Wootton), iii, 329, $331,332,333$

Stukeley, Nich. de, iii, 22 ; Mrs. iii, I 45, 246; sce also Stivecle

Sturges, Barnard C., iii, 424

Sturmere (Essex), i, I 84

Sturminster, ii, 352

Style, John, iii, 325 ; Oliver, iii, 449: Thos., iii, 325

Styrop, Rich., iij, I45

Styvecle, sea Stivecle

Styventon, see Stevington

Suckling, Thos., ii, 289

Sudbury (Eaton Socon), iii, I80 : man., iii, $193 n, 195$

Sudbury, Joan de, iii, I95; John de, iii, I95; Kath. de, iii, I95; Margery de, iii, I95: Rog. de, iii, I95; Simon, archbp., ii, 35 ; Will. de, iii, 195

Sudgible (Sudgivele), see Southill

Sudington, John de, ii, 239

Sudtone, see Sutton

Sueteman, ii, 27 I $n$

Suffolk, assessment, ii, $4^{8} n$; strawplait ind., ii, 12 I ; wool, ii, 9I

Suffolk, Sarah, ctss. of, iii, 332 ; dchsses. of, Alice, iii, 40I, 403, 415 ; Eliz., iii, 403 ; dks. of, Hen., iii, I56; John, iii, 403, 4 I 5 ; Will., iii, 403 ; Hen., carl of, iii, 332

Sulgrave, Hugh of, ii, 309, 310

Sunderland, Hen., earl of, ii, 243 ; Rob., earl of, ii, 220

Sunderland, Rev. Thos. S. J., iii, 2 I ; Lt.-Col., iii, 209, 2 I I ; Mrs., iii, 2 I I

Sundials, ii, 208, 303, $3^{8} 7$ : iii, 38 , $81,128,217,287,303,312,347$

Sundon, ii, I I 4, I 35, I 89, 306, 384 7; iii, 192; adv., ii, 387 ; char., ii, 387 ; ch., i, $315 n$; ii, 385 ; ind., ii, 384 ; mans., ii, 223 384 ; iii, 362 ; mkt., ii, 88 ; mill, ii, 385 ; Nonconf., ii, 387 ; placenames, ii, 384 ; sch., ii, I 84 ; vicarage, i, $316 n, 341$; workhouse, ii, ro5

Sundon, Will. Clayton, Ld., ii, 385

Suppethorp, Rob. de, iii, I 8 I

Surive, Pet. de, ii, II9, 122

Surrey, Eliz., ctss. of, ii, 340 ; earls of, John, ii, 340 ; Thos, ii, 301 , 340 ; iii, $13 n, 263$; see also Norfolk, dk. of

Surtees, Frances, iii, 434

Sussex, ctsses. of, Eliz., iii, 283 ; Marg., iii, 283 ; earls of, iii, $28 \mathrm{I}$; Edw., iii, 28 I ; Rob., iij, 283

Suthlingdon, sec Shillington

Sutton (Suttone), i, 335 n, 384; ii, $90,96,98, I_{3}, 201,211,237$. 246-5I : iii, I09; adv., ii, 250 : char., ii, 251 ; ch., i, $335 n$; i, 248 ; earthworks, i, 300 ; ii, 246 ; mans., ii, $238,246,250$, 256,257 ; pack-horse bridge, i 301: ii, 246; place-names, ii, 246 ; rector, i, 328 ; sch., ii, 184 ; vicarage, $\mathrm{ii}_{1} 246$
Sutton, Drew de, iii, 247 ; Eustace de, iii, 247 ; Sir John de, ii, 364 ; Marg., ii, 364 ; Olimpas of, ii, 279 ; Oliver, see Lincoln, bps. of ; Rich., iii, 259 ; Rob. of, ii, 279

Sutton Park, ii, I97, 246

Swaftham Prior (Cambs), i, 365

Swanbourne (Bucks), i, 370 ; vicar, iii, 4 I 8

Swann, Rev. E., ii, I 74

Swanton, man. (Harrold), ii, 83; iii, 66

Swart (Sweart), Ethelwine (Ailwin), ii, 21 ; iii, I28, 297

Swathyng, Marg., iii, 220 ; Thos., iii, 220

Swayne, Thos., ii, 330; see also Sweyn

Swaynes Orchard (Wootton), iii, $331 n$

Sweart, see Swart

Swell, the (Colworth), iii, 89

Sweyn, ii, 20

Swcyn, Hen. le, ii, 212 ; Will, iii, 98; see also Swayne

Swift, Will., iii, 442

Swifte, Sir Fran., iii, 3 I

Swinburn, Ad. de, iii, 78

Swineshead, ii, 7I ; iii, I23, I36, I68-70; adv., iii, I70; char., iii, I 70 ; ch., iii, I69; man., iii, I 34, I68; Nonconf., iii, I68; sch., ii, I 84 ; Three Horse Shoes Inn, iii, 168

Swineshcad, Isabella de, iii, I68; Ralf de, iii, I68, I70. Rob de iii, I68; Walt. of, i, 397 ; iii, I 68,169

Swinford (Swynford), Eliz., ii, 248 ;

Sir Thos, de, ii, 87: Thos, ii, 248

Swynerton (Swynnerton), Hugh, ii, 329 ; John de, iii, 456

Sydney, see Sidney

Syer, Rev. Thos., iii, $2 I_{4}$

Sykes, -, ii, 374

Sylam, Agnes, ii, 363 ; Eliz., ii, 363 ; Joan, ii, 363 ; John, ii, 362 , $363,366,373$; Mary, ii, 363

Symcotts, - , ii, 277

Symgw yn, ii, 332

Symond, Ad., i, 402

Symonds, Thos., iii, 250

Synnot, Henrietta L., iii, 419

Syon Ábbey (Midd.), ii, I5I ; iii, 394

Taddy, Edw. G., ii, 222, 242, 25I ; Rev. John, ii, 222, 25 I

Tadelowe, John, ii, 2 I $3 n$; Maud, ii, 2 I $3 n$

Tadlow, man. (Cambs) i, 392, 393

Tags End (Wootton), iii, 328, 329

Taillebois (Taillebosc, Talgebosc, Tallebosc), Azelina, ii, 215,216 , $232,271,275,304,305,322$; iii, I99, 253, 344, $3^{8} 3$; Cecily, ii, 257: Elias, ii, 257, 258 ; Ivo, ii, 22: iii, 206, 390; Ralph (Ralf), i, $297 n, 312$; ii, I 53, 2 I 5, 232, $248,27 \mathrm{I}, 275,28 \mathrm{I}, 305,306 n$, $321 n, 322,361$; iii, 9, 37, I 7 , $199,203,206,253,344,383,402$

Taisso (Taissel), Rob. de, iii, I86 : Rog. de, iii, I86; Wimund da, iii, I8I, I 86

Talbot, Lady Anne, ii, 340: Lady Beatrice, ii, $224 n$; Lady Eliz., ii, 332

Talbot, Eliz., ii, 223; Hugh, iii 343: John, see Shrewsbury, earl of ; Rich., ii, 223, 227; iii, I 59 ; Rob., iii, I59; Thos., iii, I59: Will, , iii, 343
Talgebosc, Tallebosc, see Taillebois Tamiseforda, see Tempsford

Tanfield, Clem., ii, 227, 238, 262 ; Eliz., ii, 227; Fran., ii, 227 ; Rob., ii, $228 n$; Will., ii, 227 ; fam. ii, 262

Tanners, Dr., ii, I 70

Tanqueray, Chas., iii, 9 r

Tansley, Alf. J., ii, I20

Tarleton, Alf. H., iii, 276

Tartlett End (Cranfield), iii, 275

Tasciovan (Tasciovanus), coins of i, I73

Tattenhoe (Bucks), iii, 302

Taverner, Fran., iii, 328

Tavistock, mqss., of, ii, $64,69,70$, I87; Fran., iii, 290; Will., ii, 43,61

Taylor (Taylard), Alice, ii, 244 ; Anne, ii, 238 ; jii, 329 ; Cath. (Kath.), ii, 239; iii, I30, I 50 , I98; Eliz, iii, I26, I50; F. G iii, 278 ; Geoff., iii, I 50 ; Humph., ii, 238; John, ii, 238, 244 ; iii, 3I, 50 ; Sir Laur., iii, I 50 : I.aur. ii, 238 ; Mich. A., ii, 204, 265 Nath., ii, $54 n$; Nich., iii, 54 329: Rich., ii, 43, 55, 28I $n$ iii, I 26, I 30, I98: Rob, ii, 241 iii, 43, 80, I 16, 293, 325; Rog. ii, 238, 358; Sim., iii, I 54, 322 Thos., iii, 126, I $30,131,132$ Ursula, iii, I30, I 31 , I 32 ; Will., ii, 43,238 ; iii, 130 , I50; W. J. iii, II6; Mrs., ii, I $82 n$; -, bel founder, ii, 2I3, 292, 319: iii, $25,68,121,179,255,278,288$, $304,334,342,37^{8}, 382,385$, $393,398,420,454$

Tebault, see Theobald

Tebb, Jonathan, iii, I 57

Tebworth (Chalgrave), iii, 343, 345 347,438 ; chant., iii, 349 ; chap. i, $318,325 n$; iii, 349

Teken, Will de, iii, I $39 n$

Temesforda, see Tempsford

Templars, i, 3 I $5 n$, 3.49, 362, 366 394; ii, 144, 216; iii, II, $59 n$ $66,67,70,78,90, \mathrm{I}_{34}$, I 54 I $58,165,315,318,324,437$ Aimery, master of, iii, $1(1,5$ Rob. de Sanford, master of, iii, $f, t$, 'Templars' Lands (Pertenhall), iii, I 54

Temple, Sir Will., ji, 275

Temple Hills, man. (Sharnbrook), i, 394 ; iii, 90

Tempsford, i, 273, 284, 297, 30\% $346 n, 403$; ii, I 9, 20, 44, 65, II 3, I 3o, 20I, 206, 226, 25I-5, 276 ; iii, 209: adv., ji, 254: chant., i, 329; ii, I5I, 233, 245, $254,255,258$; char., ii, 255 ch. i, 315 11 ; ii, 25I ; earthworks, i, $280-2$; ii, 251 ; fishery ii, 253 ; mans., ii, $25 \mathrm{I}$; mills, ii 253: Nonconf., ii, 255 ; prehist. rem., i, 145; ii, 251 ; place-names, ii, $25 \mathrm{I}$; rector of, $i, 34 \mathrm{I} n, 342$ Rom, rd, at, ii, 4 ; schs., ii, I 50 , I 5I, I 84, 25I

Tempsford Hall, ii, I97, 251

Temysford, see Tempsford

Teobbanwyuthe, see Tebworth

Terle, Thos., iii, 332

Tesserae, ii, $7,12,13$, I 5

Tessington, Rich., iii, I 39

Tetbald, see Theobald

Tetbaud, iii, I 8 I

Tetebury, Rich., iii, 137; see also Tutbury

Tethbald, see Theobald

Tetricus, coins of, ii, 7

Tettesworth, John, iii, 456 ; Nich. iii, $45^{6}$ 


\section{A HISTORY OF BEDFORDSHIRE}

Tetworth (Hunts), ii, 7I, 226, 228, 230 ; sch., ii, 182

Tewkesbury, abbot of, i, 320, 362

Teye (Teyes), Isabel, iii, 14 ; Walt de, iii, I 4,45

Teynturer, Laur. le, iii, $358 n$

Thame Sch. (Oxon), ii, I62, 164 , 173

Thanet, earl of, iii, 44

Thatchford mill, ii, 355

Thaxted (Essex), 1i, 24I

Thayne, Jas., ii, 355

The, John de la, ii, 145

Thebrugg, Rog. de, i, 352

Thebworthe, see Tebworth

Theed, Eliz., iii, 198; Hen., iii, I99: Will., iii, 198, 199

Theistic church, iii, 3 I

Themes, Nich. de, iii, 2 I9

Theobald (Tebault, Tethbald, Tetbald), ii, 3 I 5 ; iii, 85

Theobald, Fran., iii, 29r ; Rich., ii, $204 n$

Theodore, archbp., i, $34^{6}$

Thimelby, Eliz., iii, 291

Thirning, Sir Will., iii, 130

Thody, Oliver, ii, 268

Thomas (Cancellarius), i, 400 n

Thomas, Rob., iii, I 8

Thomlynson, see Tomlinson

Thompson, Cath., iii, 396 ; Dorothy, iii, 397; Edw. H., ii, 282 ; Eliz., iii, 328; Geo., iii, I95; Jane, iii, 398 ; Sir John, iii, 395 ; John, iii, $74,286,307,333$, 395-8 ; Judith, iii, 395 ; Mary, iii, 332 ; Maurice, iii, 296 ; Rob., iii, 395 ; Sam., iii, 332 ; St. John, iii, 395, 396, 398; Ursula, iii, 396,398 ; Sir Will, iii, 332 ; Will., iii, 328 ; 一, ii, I35

Thomson, John C., iii, 242 ; Will., iii, I07

Thorn (Houghton Regis), ii, 8 . iii, 390

Thornbury, man. (Houghton Regis), iii, 39 I

Thorncote (Northill), ii, 92 ; iii, 242 , $248,250,25$ I

Thorne, Edw., ii, I60, I6I ; Giles, i, $337-40,342,343$; iii, 28 ; Obed, ii, 320; Will., iii, 28 ; Mrs., i, 338,339

Thorney Abbey (Cambs), i, 312, 3I4, $315 n$; iii, I 24, 125, I27, I 43, I 79, I 88, 396

Thornton, Col. Godf., iii, 231, 246 , 247 ; Godf., ii, 252 ; iii, 231 ; Harry, ii, I89; iii, 205 ; Rob., iii 231, 247; Steph., iii, 231 ; Thos. de, i, 370 ; Col. Will., iii, 231 Will., ii, 228; Miss, iii, 419; Mrs., iii, 228

Thorowgood, Sir John, iii, $380 n$ : see also Thurgood

Thorpe (Thorp), Geo. de, ii, 382 383 ; John de, i, 398 ; ii, 382 $3^{8} 3$; Matilda, de, ii, 382 ; Rob., ii, $382,3^{8} 3$; Will. of, i, $3^{8}$ I

Thrale, Agnes, ii, 359

Thrayle, Mich, ii, 39

Three Counties (Arlesey), ii, 265

Three Counties Asylum, ii, 300

Threkeston, Rich., iii, 30

Threlkeld, Launcelot, iii, 91 ; Laur. iii, II 8; Marg., iii, 91, II8, InI

Thrift Wood (Silsoe), ii, 326

Thring, Llewellyn C. W., ii, I79

Throckmorton, Bromsall, iii, 247 Sir Geo, iii, 220; Geo., iii, 219 Judith, iii, 183 ; Rich., iii, 183 ; Rob., iii, 219

Throckmortons, man. (Roxton) iii, 220
Thrupp, Fred, iii, 30

Thrupp End (Lidlington), iii, 305

Thrupp End Farm (Lidlington), iii, 306

Thuand, fam., iii, 210

Thurald, John, ii, 253

Thurgood, John, iii, 258 ; Nich., iii 258, 259; Thos., iii, 258 ; see also Thorowgood

Thurkytel, ii, I9

Thurleigh, i, 384 ; ii, 44 , I I 5,342 ; iii, I $5,31,34,51,60,69,82$, I $04-9$, I $26,136,143,146,147$ I50, 210, 212, 226, 235, 283, 331 ; adv., iii, ro8; char., iii, Iog; ch., i, $287,315 n$; iii, Io7 College Farm, iii, I04; earthworks, i, 287,308 ; iii, 104 ; Jas. I. at iii, Io7; mans., i, $325 n$. iii, I04; mills, iii, I05; sch., ii, I 84 ; iii, 109

Thurleigh Hall, iii, 104

Thurley, see Thurleigh

Thurston, Thos., iii, 4 I 5

Thynne, Lady Sophia, iii, 132 Lds. : Geo., ii, 341 ; John, ii $182 n, 185 n, 339,341,343$

Thyone (Thynn), A. C., iii, 326 , 328 ; Fran., iii, 363, 402 ; Fran. J., ii, I 47,34 I, 344; iii, 293 296 ; Hen. F., see Carteret, Ld. John, see Carteret, Ld.

Tickford Moulsoe, iii, 27

Tickmarsh (Tichemersh), Hen. de iii, 57 ; Isabel de, iii, 57 ; Maud de, i, 390

Til, riv., i, 289, 29 I ; iii, I 32, I 7 I, I 76 Tilbrook (Hunts), ii, 71, 81, II 5 ; iii, $59 n$, I $15 n, 123,168$, I 7 I- 5 adv., iii, I75; char., iii, I 75 ch., iii, 156, I 73; Hardwick Farm, iii, I $7 \mathbf{I}$; Manor Farm, iii I 71 ; mans., iii, 156, I7I ; mills, iii, 171; Nonconf., iii, 171; rector of, i, $338,342 n$

Tilbrook, Rohese de, iii, 172

Tilbrook Grange, iii, I 7

Tilbrook Hall, iii, I 7

Tilebroc, see Tilbrook

Tile industry, ii, I1 7 ; iii, I 57

Tiles, r5th cent., iii, 450 ; Rom., ii, $7, \mathrm{I}$ I, 15

Tileword, see Tilsworth

Tillebrok, see Tilbrook

Tillisword, Tillsworth, see Tilsworth

Tilly (Tilley), Hen., iii, 224 ; Matilda, iii, 224 ; Rob., iii, 3

Tilsworth, ii, 4I, II4, 375 ; iii, $336,400,432-5$; adv., iii, 435 ; char., iii, 435 ; ch., i, $315 n, 324 n$; iii, 434 ; earthwork, i, 291 ; man., iii, 433 ; manor-house, iii, 432 ; vicarage, $i, 316 n$

Tilwick (Ravensden), iii, 212, 2 I 3

Tilwick Farm (Ravensden), iii, 210,212

Tinden (Finedon, Northants), ch., i $37^{8}$

Tingey, Hen., ii, 253 ; Will., ii,

Tingeys House (Tempsford), ii, 253 Tingre, Tingrei, Tingriff, see Tingrith

Tingrith, i, $335 n$; ii, II 4,324 ; iii $286,336,382,435-8$; adv., iii, 438; Castle Farm, iii, 435; char., iii, 438 ; ch., i, $324 n, 357$ iii, 437 ; ind., iii, 435 ; manor house, iii, 435 ; mans., iii, 435 : sch., ii, 184

Tingrith (Tingry), Cecilia de, iii 442 ; Gilb. of, i, 321 ; Nich. de, iii, 395 ; Pet. de, iii, $436 n, 437$; Thos. de, iii, $436 n$; Will. de, rii, 442
Tingritb Mead (Westoning), iii, 455

Tingry, see Tingrith

Tipper, Will., iii, II $7 n, 173,3^{85}$

Tipper and Dawe, iii, I 73

Tipping (Tippinge), Anne, Lady, iii, 376; Sir Thos., iii, 376 Capt., iii, 242

Tiptot, Eliz., ii, 385 ; John (de), ii $3^{84}, 3^{85}$; Marg., ii, 385 ; Millicent, ii, 385 ; Rob. (de), ii, 384,385

Tirell (Tirrell), see Tyrrell

Tissington (Derb.), chap., i, $372 n$

Tithe Farm (Stevington), iii, $\mathrm{IO}_{4}$

Tithe Mills (Clifton), iii, 258

Tivill (Tyville), Elena de, ii, 334 : Hugh de, ii, 333, 334 ; Ralph, ii, $294,297,333$

Tocke, see Tooke

Tocotes, Eliz., Lady, ii, 296; iii, 230 ; Sir Rog., ii, 296 ; iii, I 87 , 230, 326

Tod, Gilb., ii, $26 n$

Toddenham (Tudenham), John de, iii, I5; Kath. de, iii, I 5, 330 Marg. de, iii, I5; Rob. de, iii, I 5 330; Thos. de, iii, I 5 ; fam., iii, 329

Toddington, i, $176,286,325 n$, $33^{8} n, 344,367 n, 400,402$ : ii, $2,45,56,58,84,114,277$ $363 n$; iii, $336,346,368,438$ 47,451 ; adv., iii, $44^{6}$; A.S rem., i, I8I ; iii, 439; chant. i, $325 n$; iii, 446 ; chaps., iii, 446 char., ii, I $85 n$; iii, 446 ; ch., i, $185,315 n$; iii, 439,444 ; farms, iii, $43^{8}$; hosp. of St. John Baptist, i, 330, 33 I $n, 368,402-3$; ind., iii, 439 ; Jas. I. at, iii, 440 ; mans., i, $287 n$; iii, 380,383 439 ; mkt. and fairs, ii, 88 ; iii 44I; mills, ii, 278 ; Monmouth at, iii, 44 I ; place-names, iii, 439 ; plague in, iii, 440; Portionaries, the, iii, 444 ; prehist. rem., i, I69. I74: iii, 439 : Rom. rem. at, ii I 4-I 5 ; Rom. rd. at, ii, 4 ; schs. ii, 184,185

Toddington Park, iii, 439

Toddington Place, iii, $44^{\circ}$

Todeni, see Tony

Tofte, The (Tofts), man. (Sharn brook), iii, 88.92, 95

Tofte, Will., iii, 90

Tole, Rob., iii, 80

Toller, Ralph le, iii, 22

Tolleshunt Major (Essex), ch., i, $382,3^{84}$

Tolls, iii, 356, 362, 408

Tolthorp, Rob. de, iii, 139

Tomlinson (Thomlynson), Alice, ii, I60: Thos., ii, I60

Tompion, Thos., iii, 250

Tompson, see Thompson

Tongue, W., ii, I 89

Toni (Tony, Todeni), Agnes de, iii, 426; Rob. de, iii, II I I 49, 426 ; Rog. de, ii, 3 I5; Will. de, iii, I I2 ; fam., iii, 57

Tooke (Tocke, Took), John H., ii, 64 ; iii, 20 ; Will., iii, I 87

Top End (Renbold), iii, 2 I 4

Toplers Hill (Edworth), ii, 209:

Rom. rem., ii, 7

Torfeye, see Turvey

Torksey, Walt, of, i, 399

Tornai, see Turvey

Torrington, Marg., Lady, iii, I45: Geo., visct., ii, $61,69,238,291$, 346 ; iii, $145,246,253,255,258$, 260,261

Torveie, see Turvey

Tostig (Tosti), earl, ii, $21 n, 153$, 203,2 I $6,226,321$ : iii, 248 


\section{INDEX}

Totternhoe, i, 336 ; ii, 84, II 4 , 356 ; iii, 69, $294 n, 336,368,430$, 447-5I ; adv., i, 376 ; iii, $45^{\circ}$ : char., iii, 45I: ch., i, 3I5 n, 37 I $n$; iii, 450 ; Cross Keys Inn, iii, 447 ; ind., iii, 447 ; man., iii, 37 I, $44^{8}$; mill, iii, 447 ; placenames, iii, 448 ; prehist. rem. i, I 72 , I 74 ; iii, 448 ; quarries, iii, 373, 448 ; Rom. rem., i, I 72 : ii, I5: iii, 448; sch., ii, I85; vicarage, $i, 3$ I 6 n

Totternhoe Castle, i, 293, 294 ; iii, 448

Totternboe Downs, ii, 15

Totternhoe Hill, iii, 350 ; prehist. rem., i, I 70

Tottman, Mary, ii, $24 \mathrm{I}^{\circ}$

Tovi, housecarl, iii, 6I; priest, iii, 126

Towcester (Nortbants), ii, 2, 3 46,49

Tower, Chris., ii, $282 n$

Tower Hill (Sandy), Rom. rem., ii,

9, 10

Towersey, Rev. - , ii, I 72, r73

Toweslonde, John de, ii, 257

Town Close (Cardington), iii, 238

Towne, Rev. Leonard, iii, I8 7

Town Farm (Eversholt), iii, 378

Town Field (Maulden), iii, 316

Townley, 一, iii, 142

Town Row (Maulden), iii, 3r6

Towns End House, see Sharnbrook House

Townsbend (Townsend), Eleanor, ii, 382 ; Rog., ii, 382; Thos., ii, 382 ; Will., iii, 37

Towse, Will., iii, 133

Tracy, Hugh, i, 402

Trailly, bar. of, iii, 36, 54, I Io, I75, 219

Trailly, hon. of, iii, 36,257

Trailly, Albreda de, iii, 243, 257 . Eleanor, iii, 36, 56, 176; Geoff. de, iii, 54, I75, I 76, I 79, 243 , 257: Joan, i, 357; Sir John, i, $329,357 n, 403$; iii, 244, 250; John, iii, 5I, 54, 55, I IO, I 76 , I 77, I 79, 2 II $, 243,244,330$; Mary, iii, I76, 243; Maud de, iii, I 76, 243 ; Nicb. de, i, 361 ; $362 n$; ii, 233, 243; Norman de, iii, 55; Sir Reg., iii, 244, 250; Reg., iii, 5I, 2 I I, 243, 330 ; Reynold, i, 329, 403; Walt. de, iii, $36 n, 55,56,74, \mathbf{1} 75, \mathbf{I}_{76}, \mathrm{I}_{79}$, 2 I I, 244 ; fam., iii, 85,249

Traillys Close (Ravensden), iii, 2 I I

Trailly's Manor (Ravensden), iii, 2 II

Tranecroft (Sharnbrook), iii, 90

Trawles Close (Wootton), iif, 333

Trayles Fields Farm (Ravensden), iii, 210,211

Tregoz, John, ii, 8o ; iii, 452; Mabel, iii, 452

Tresham, Sir Thos., iii, 354

Treswell, Rob., iii, II 3

Tretheny, Hen., ii, $38 \mathrm{I}$

Trevanion, John B., ii, 357

Trevor, Lady Ann, iii, 48 ; Lds. iii, 54; John, iii, 45; Rob., iii, 37 ; Tbos., iii, 45, 47, 48, 55, 96

Trevor, Cath., iii, 437 ; Eliz., iii, 437 : Sir John, ii, 46 n, 60 ; It.Col. John, ii, 7o; John, iii, 45 . Capt. Lewis, iii, 48; Mary, iii, 436 ; Rob., iii, 436; Sir Thos. see Trevor, Ld. Thos. ; fam., ini,

37
Trevor-Battye, Capt. C. E., iii, 435,

Tribal or Military earthworks, i, 269

Trice, 一, ii, 277
Triket (Tricket), Bald., iii, 9o, 94 ; Sim., iii, 90 ; Will., iii, 90 ; W., $315 n$

Trikynham, Walt. de, iii, 60

Trimer, Will., ii, 304

Trinity College (Camb.), ii, I79, $180,299,302,303,304,335$; iii, $59,61,63,80$, 100, I39, I85, 221 , $222,237,374,375,458$

Trinity College (Oxford), ii, I7I, 374

Trinity Hall (Camb.), ii, 380; iii, 349

Tristran, Lawr., ii, 304

Trowley Bottom (Flamstead), ii, 375

Troy Hall (Cranfield), iii, 275

Trubleville Hen. de, iii, ror

Trumpington, man., see Perots

Trumpington, Blanche, Lady de, iii, 230 ; Eleanor, iii, 230 ; Giles de, iii, 230, 248 ; Marg., iii, 230 , 248; Maud de, iii, 230; Sir Rog. de, iii, 230 ; Rog. de, iii, 230 ; Sir Walt., iii, 230

Trusse, John, iii, I 8

Trussell, Isabel, iii, 7 I ; Marg., iii, 299 ; Sir Will., iii, 7I ; Will., iii, 299

Tryon, Eliz., iii, 102 ; Sir Sam., iii, 102

Tryvet, Eliz., ii, 360 : Thos., ii, 360

Tubb, Will., ii, r65

Tucker, Jos., ii, $184 n$; iii, 6o, 80 ; J., iii, I 45

Tuckey, Will., iii, 432

Tudenham, see Toddenham

Tudingetone, see Toddington

Tudor, Jasper, ii, 39

Tuke, Geo., iii, 78, 102

Tumuli, see Burials

Turbert, iii, 54

Turchil, iii, 120, 424

Turford, Thos., ii, 309

Turgis, iii, 9o, 146, 286, 375, 396,

Turk, Beatrice (Beatrix), ii, $256 n$, 354; Joan, ii, 354; Rob., ii, 256 n

Turneley, John, iii, 349

Turner, see Turnor

Turney, Thos., iii, 278

Turneyes Ground (Leighton Buzzard), iii, 404

Turnball Mill, i, 352

Turnor (Turner), Anne, iii, I3I ; A. J., ii, r99, 200 ; Sir Chris., iii, I 44 ; Chris., iii, I44, I 48 ; C. H., iii, I48; Sir Edm., iii, I45, I49; Edm., iii, I 44 ; 'Sir Edw., iii, I44, I49; Jacob, iii, 125; John, iii, I 45 ; Sam., iii, 427 ; Sir Thos., iii, I 31

Turnor Almshouses (Milton Ernest), iii, 149

Turold, priest, iii, 384

Turri, Elias de, iii, 428: Lavinia de, iii, 428

Tursa, iii, r69

Turstin (Turstan), ii, 267, 322 ; iii, $76,83,85,248$

Turvey (Turveia), i, 335, $380 n$; ii, $40,60 \mathrm{n}, 7 \mathrm{I}, \mathrm{II} 5, \mathrm{I} 47, \mathrm{I} 87,324$, 326 ; iii, 34, 49-5I, 70, 104, 109-I7, I 75, I83; adv., i, 3I $5 n$; iii, II6: almshouses, iii, I1 7 ; char., iii, I1 7 ; cb., i, 31 $5 n$; ii, 72 ; iii, II5: farms, iii, III ; mans., iii, IIO : mills, iii, II2, II 4 ; placenames, iii, IIO; rents in, ii, 132 ; sch., ii, 185: Three Fishes Inn, iii, 109

Turvey Abbey, $\mathrm{ii}, 188$; iii, ro9, 1 I 1 , II $4 n$, II 5

Turvey Hall Farm, iii, Iog, I I I
Turvey House, ii, I 47 ; iii, 5I, 109 I I I, 1 I 6

Turvey Lees, iii, I I

Turvey Lodge Farm, iii, 1 i

Turvey Old Hall, iii, rog

Turvyll, Will. de, ii, $34 n$

Tutbury, Kath. of, i, 389,390 ; sec also Tetebury

Tutty, - ii, 344

Tuward, Will., iii, 138

Twamley, J. H., iii, I 32

Twinberrow, Miss, iii, 2 I I

Twylesworth, see Tilswortin

Tyley, Rev. Jas., ii, $3^{84}$

Tyndale, Mary, ii, 382 ; Will., ii, 382

Tyngree, Tyngreve, see Tingrith

Tyringham, Ant., iii, 77 ; Eleanor, iii, 77 ; Geoff., iii, 2 I 2 ; John (de), iii, 77,83 , I50, 212 ; Rob., iii, 64, 67, 77: Rog., iii, 212 : Sim. de, iii, I50; Sir Thos., iii, 57, 58,83 , I 39 ; Thos., iii, 77

Tyrington, Joan, iii, 339 ; Sir Will. de, iii, $34^{\circ}$; Will., iii, 339

Tyrell's End (Eversholt), iii, 375

Tyrrell (Tirell), Alice, iii, 224 ; Anne, ii, 78; Dorothy, iii, 78 :

Edw., ii, 385; iii, ro6; Geo., iii, 9r : Joan, iii, 9 r ; John, iii, 78 ; Pet., iii, 224; Ralph, iii, 224, 225 ; Rich., ii, 385 ; iii, 78 , Iо6 : Thos., iii, 73, 224, 225 ; Will., iii, 78 ; fam., iii, 126

Tyrwhitt, Rob., iii, 90, I06, I60 $n$

Tyville, see Tivil

Ufford, Eliz., iii, I 3 ; Rob. (de), i,

$382 ; \mathrm{iii}_{1}$ I 3

Ulf, iii, 426

Ulf, Thos., i, 399

Ulfech, iii, 195

Ulfkytel, ii, 20

Ulmar (Almar, Wolfmar), i, 300 ;

ii, 2 I 5 ; iii, 92 ; (priest), iii, 38

Ulsi, ii, 263; see also Alsi

Ulster, Matilda, ctss. of, iii, 403

Ulverton, Rich., i, 402

Ulveva, iii, I62

Ulvric, see Alvric

Umfreville (fam.), iii, 43

Underhill, Thos., iii, $24^{8}$

Underwood, Thos., ii, 59, 166

Will., iii, 462

Unitarians, iii, 9, 42 I

University Coll. (Oxford), iii, 275

Upbury man. (Pulloxhill), ii, $37^{8}$

Upbury moat, ii, $376,37^{8}$

Up End (Kempston), iii, 297

Up End (Stagsden), iii, 96

Up End Wood (Stagsden), iii, 96

Upper Fields (Radwell), iii, 305

Upper Ossory, ctss. of, ii, $63 n$; earls of, ii, $61,64,69,70,1_{4} 6$; iii, 268, 272, 288, 291, 306, 307, 318, 319: John, iif, 286, 291, 3Io, 3I I, 3I 7 ; Ld., iii, $27^{\circ}$

upton, Chas., ii, 44 ; Rich. de, iii, 4 I9 $n$; Will. de, iii, 4 I9

Upwood, Mary, iii, 26I ; Thos., ii, 280 ; Thurgood, $\mathrm{iii}, 26 \mathrm{I}$

Urlin, Geo., ii, 385; Sir Sim., iii, 268

Urns, cinerary, see Burials and Burial Urns

Urry, - ii, $45 n, 46$

Ursel, Will., iii, I $48 n$

Utcoate (Hudicote) Grange (Wo-

burn), iii, $45^{8}, 46 \mathrm{r}$

Utteford, Rob. de, iii, 9 r

Vale Farm (Lidlington), iii, 305

Valence, bps. of, $i, 400$ 


\section{A HISTORY OF BEDFORDSHIRE}

Valence (Valencia), Agnes de, iii, 229: Aymer de ii, 223, 275: Joan de, ii, 223: Will. cle, ii, 223 Valence End Farm (Dunstable), i, I 70

Valery, Guy de, iii, 339

Vardon, Alf., ii, 199

Varney, see Verney

Vaseys, man. (Gt. Barford), see Veseys, man.

Vaudey, monastery, i, $367 n$

Vaughan, Eliz., G., ii, 304 ; Geo., iii, 246 ; Mrs. W., ii, 304

Vaux, Lds., iii, 51, 101, I30, I50, I51; Edw., iii, I9I; Hen., iii, 51; Nich., iii, 45, 51, 77, 130 : Thos., iii, 45, 5I, 77, 19I; Will., jii, 131

Vaux, İleanor de, ii, 35I ; Eliz., iii, 77 : Ernest, see Harrowden, Ld. ; Geo., iii, 78 ; John, ii, 44 ; Maud, ii, 354 ; ii, 422; Sir Nich., see Vaux, Ld.; Nich., iii, I26 ; Rob., iii, 456; Sir Thos., see Harrowden, Ld. ; Thos., ii, 69 ; iii, 432, 456 ; Sir Will. de, ii, 35I, 354 ; iii, 51 ; Will., ili, 201, 405, 422 ; fam., iii, $77,235 n, 36$ I

Vauxes, man., see Ocle-cum-Clapham

Vavasour, John, iii, 299

Veale, Rich., ii, 380 ; see also Viel

Vendôme, count of, ii, 36

Ventris (Ventriss), Sir Chas., ii, 44, 266, 268, 343 ; Sir Fran., ii, 268, 278,343 ; Fran., ii, 268, 343 ; iii, 49; Henrietta, ii, 268 ; Hen., ii, 342,343 ; iii, 61; Joan, ii, 268 ; John, ii, 59, 268, 342, 343, $343 n$; Mary, ii, 268, $343 n$; iii, 61 ; Will., ii, 342

Ver, riv., i, 29, I46, 15I; ii, 314

Vere, Aubrey de, i, $378 n$; ii, 23 ; Eliz., iii, II5; John de, see Oxford, earl of ; fam., iii, $459 n$

Verey, J. G., ii, I95

Verney (Varney), Cath., iii, 432 ; Sir Ralph, iii, 148; Will., ii, r64-5; iii, 432

Vernon, Lady Harriet, iii, 441

Vernon, Chris., iii, I1 3 ; Henrietta, ii, 277 ; Hen., ii, 278 ; iii, $441 n$; Rich., ii, 63; iii, 20 ; 一, ii, 64, 192

Verulam (town), see St. Albans

Verulam, Alice, vsctss., ii, 233

Vesey, Hen. Bromflete, Ld., sce Vessy

Ves ys (Vaseys), man. (G.t. Barford), iii, 181

Vessy (Vesey), Hen. Bromflete, Ld., iii, 9 r, I1 8

Viel, Rev. John de, iii, r28; see also Veale

Villiers, Philippa, iii, 97 ; Will., iii, $97,4^{\circ} 4$

Vine Farm (Langford), ii, 203

Vinegar Hill (Northill), iii, $25 \mathrm{I}$

Vipont, Ida, ii, 230 ; Isabel, ii, 230; Rob. de, ii, 230

Vitella de Ferentino, Pet. de, i, 32 I

Vivian, Chris., iii, 58 ; Humph., iii, 58

Vivian's Croft (Studham), iii, 432

Vivonia, see Vyvon

Vollans, Will., iii, 95

Volunteers, ii, 70

Vyall, John, i, 395

Vynter, John, iii, 231 ; Sir John, ii, $20:$

Vyvon, Cicely de, ii, 351 : Heloise de, iii, 7 I ; Hugh de, iii, 71 Joan de, ii, 35r, $352 n$; Mabel de ii, 351 ; Maud de, ii, 351 ; Sybil de, ii, 351; Will. de, ii, 35I
Wade, Hester, iii, 198 ; Rev. Hugh, iii, 198

Wade-Gerv, Rev. Rob., iii, I89: Will., iii, ro5, I90; W. H., i, $385 n$; iii, I05, 198 ; fam., ii, 23 ; see also Gery

Wadehelle (Wadelle), par., see Odell

Wadelowe, see Wadlowe

Wadhull, fam, see Wahull

Wadlowe (Wadelowe), Hugh de, iii, $44^{2}$; John de, iii, 442

Wadlowes, man. (Toddington), i, $37^{6}$; ,iii, 380, 442, 454

Wadsworth, John, iii, I56

Waferer, Anne, iii, r $3 \mathrm{r}$; Arden, iii, 129, 130 ; Eliz., iii, 130 ; Jas., iii, 130

Wagstaffe (Wigstaffe), Eliz., iii, $2 \mathrm{r}_{4}$; Geo., iii, 224 ; Hen., ii, 363 ; iii, $224 n$; Jane, iii, 84 John, iii, 2I 4,282 ; Rich., ii, 55 iii, 84 ; Will., iii, 224

Wahull, bar., see Odell, bar.

Wahıll, par., see Odell

Wahull (Wodhull, Woodhull), Agnes, ii, 234, $234 n$; iii, 70-2 ; Albreda, ii, $283 n$; Alice, iii, $70 n$; Ann, iii, 72 ; Ant., ii, 234 : iii, 72; Cecily de, iii, $70 n$ Eleanor, ii, $204 n, 234$; iii, $7 r$ Eliz. de, ii, $204 n, 234$; iii, 71 72,384 ; Fulk de, iii, 72 ; Heloise de, iii, 71; Isabel, iii, 71; John de, ii, $204 n, 234 n, 283,326$ : iii, $70-4,76,82,118,285$; Mary de, iii, $70 n$; Mich. cle, iii, $70 n$; Nich. de, ii, 234; iii, 7I-2; Rose de, iii, 7o; Saer (Saher) de, ii, 234 ; iii, 69, 71-4, 76; Sibyl, $i$, $394 n$ : Sim. de, i, 371 I $n 394 n$, ii, 235 ; iii, 7I, 321, 323; Thos. de, iii, $70,71, \mathrm{xO}_{4}, 105,384$; Walt. i, 37I $n$; ii, 26, 234, $234 n$ $283 n$; iii, $70-1,74,104,448$ 450 ; Will. de, iii, 211 ; fam., ii, 204 ; iii, 69, 321 ; see also Odel!

Wainright, Abigail, iii, 444 ; Geo., iii, 444 ; John, iii, 443

Wake, man (Cardington), ii, 235

Wake, Lady Alina, iii, 252 ; John Ld., iii, IOI

Wake, Alice, iii, 45 ; Aline, ii, 334 Alinor, iii, 221 ; Bald., ii, 30, 31 iii, I2, I $4,45,74,78$, IOI-2, I $36 n, 215,375$; Blanche, iii, IOI ; F.la, ii, $30 n$; iii, I2, 215 , 375 ; Eliz., iii, $\mathrm{x}_{4}, 46,375$ Hadwisa, iii, Ior: Hugh, iii, 162, 252; Ida, iii, I4, 215 Isabel, $\mathrm{ii}, 30 \mathrm{n}$; Jas., ii, 334 ; iii, 252 ; Joan, iii, $x_{4}, x_{5}, 46,215$; John, iii, ror; Marg., iii, ror Maud., iii, 45, 252 ; Sir Thos., iii, 45, 235; Thos., iii, Ior; Sir Will., ii, 64; iii, 20; fam., jii, 206, 237

Wakefield, John, iii, 2 I 6

Wakes, man. (Bromham), iii, 45

Wakes, man. (Eversholt), iii, 377

Wakes End Farm (Eversholt), iii, 375

Wascot, Eyre, iii, I59; John, iii, $2 ; 2 x$

Waldegrave (Walgrave), iii, $39 \mathrm{r}$

Waldegrave (Waldgrave, Walgravc, Walgrove), Eliz., iii, 298; John, ii, 233; Rose, i, 357 ; Warin, iii, 298 ; Will., ii, 65 ; fam., iii, 43

Walden Abbey, $i, 390,391 n$

Waldeouf, count, see Huntingdon, Waltheof, earl of

Waldeshef, John de, iii, 165

Wald grave, see Waldegrave

Waleis, see Waleys and Whalley
Waleran, Matilda, iii, 301 ; Rob., iii, $30 \mathbf{r}$

Wales, man. (Eaton Socon), iii, 198-9

Waleys (Waleis, Walley, Wally), Beatrice, ii, 354; Elcanor le, iii, 299 ; Joan, ii, $334,354,362$, 363 ; iii, $77,198 n$; John, ii, 354 362 ; iii, 393 ; Joyce, ii, 362 Rich. le, ii, I80: iii, 299: Sir Will., iii, 393 ; Will. le, ii, 334 ; iii, 393

Walgrave, Walgrove, see Waldegrave

Walker, John, ii, 194, 221; iii, 392 ; Jos., iii, 287, 325 ; Rich., iii, 4 I6; Dr. Walt., i, 337

Wallace, J. W., ii, I4I

Wall Close (Eversholt), iii, 375

Waller, Edm., ii, 42: iii, $r_{40}$ John, ii, II9, $342 n$; Rob., iii 140; Thos., ii, 349 ; - , ii, 47 ; fam., iii, r 94

Walley, see Waleys

Wallinger, John, i, 337

Wallingford, hon. of, iii, 129, 130, 150,154

Wallingford, prior of, ii, 252

Wallington, Nehemiah, ii, 42

Wallop, Eliz., Lady, iii, 405; Sir Hen., iii, 405; Rich., ii, I 79 ; Rob., iii, 405

Wall paintings, iii, 43, 108, I15, $121,127,163,164,178,295,303$

Wallud's Bank (Luton), see Waulud's Bank

Wally, see Waleys

Walsall, Fran., i, 343; ii, 245

Walsham How, Rev. W., i, $332 n$

Walshe, Walt., ii, $25^{\circ}$

Walsingham, Sir Fran., iii, 294

Walter, i, 313; ii, $271 n, 326$, $383 n$; iii, $85,120,173,206,248$, 253, 321

Walter (Wylter), Rich., ii, 157

Waltham Abbey, i, 312, 323, $348 n$; ii, 154, 155, 220, 261 $262,263,265$

Waltheof, earl, see Huntingdon, earl of

Waiton, Cecyle, iii, $24 \mathrm{r}$; Christina de, iii, 456: Hen. de, iii, 456 Sir Thos., iii, 24I ; Thos., iii, r94

Walweyn, Joan, iii, 437; Phil., iii, 437 Walweyns man. (Tingrith), iii, 437

Wanwyle, see Wavyle

Warburton, Rich., ii, 30

Ward, Hen., ii, 24I ; John, i, 403 : ii, 237; iji, 226, 250; - ii, 353

Ward, B. \& Co., ii, I27

Warden, Old, i, 270, 305, 361, 364, 365,393 ; ii, 96, I16, 197, 336 iii, $219 n, 227,245,25 x-6,259$, 26r, 264; adv., i, $361 n$; iii, 255; Celtic rem., i, r 70, 74 iii, 252 ; char., iii, 255 ; ch., i, 36r, 362, 364; iii, 254 ; crown property, iii, 271 ; forest offences, ii, r44; inclosures, ii, 96 ; mans., iii, $248,252,317$; mkts. and fairs, ii, 88 ; iii, 254 ; Park Farm, iii, 254; parson, i, 323 ; place-names, iii, 252 ; Rom rem., ii, 15 ; iii, 252 ; sch., ii 184

Warden, bar., iii, 57, 70, 85, 120, $243,252,257,258$

Warden, hon., iii, $175,176,248$

Warden, John, i, 403; iii, 57,250

Warden Abbey, i, 349, 351, 356, $36 \mathrm{r}-6$; ii, $28,43,53,60,144$, $275,277,278,28 \mathrm{r}, 283,288$ 290; iii, 7, 24, 97, 98, 107 147, $190 \mathrm{n}, 204,206,207,210$ $212,225,233,237,246,247,25 I_{1}$ 


\section{INDEX}

Warden Abbey (cont.)

$253,254,255,257,258,259,285$, $315,317,318,324$; abbots, ij, 365,$367 ;$ ii, $84,204,230,233$, 275,28 I $n$; iii, I 2, $38,97,98$, 1 47, 204, 206, $212,225,245,248$, 255, 259, 273; mill, ii, 283 ; seal, i, 365; iii, 252

Warden Hill, prehist. rem., i, I71, I 72, 280; ii, 280

Warden Park, iii, 25I, 253

Warden Tunnel, iii, 25I

Warden Warren, iii, 25

Warden Woods, iii, 25I

Wardle, Col., iii, 4

Wardone, see Warden, Old

Ware (Herts), i, $297 n$; ii, 141: iii, 203, 206

Ware, -, ii, 273

Waren fam., see Warenne and Warren

Warenne, earl, ii, zoI $n$

Warenne, Will. de, iii, 133, 134, $\mathrm{x} 68,169,171$; see also Warren

Wareton, Thos., iii, I62

Warham, John, i, 353

Warin (Warine), see Warenne and Warren

Warison, John, ii, $24^{8}$

Warner, iii, Ix 4

Warner, Edw., ii, 326, 333; John, iii, $94,326,345,383$; -, ii, 265 ; iii, 446,450

Warre, Anne, iii, 410; John la, iii, I62; Rog., iii, 410; see also De la Warr

Warren (Waren, Warin(e)), Anne, ii, 373 ; Elinor, i, 389, 390 ; Eliz., ii, $328,331,332$; Humph., ii, 329,332 ; John, iii, I 33,142 ; Thos., ii, 328, 331, 332; Will. iii, 92 ; fam., ivi, $3_{22}$; see also Warenne

Warren Farm (Millbrook), iii, 316

Warren Farm (Ridgmont), ii, I 41

Warren Hill (Suff.), i, I82 $n$

Warren-Vernon, Eliz. G., iii, 439, $44 I, 443$

Warwekhyll, John, iii, $45^{\circ}$

Warwick, earls of, i, 373 ; ii, 37 327 ; Rich., iii, 403 ; Thos., i, 373 . Will., iii, 129

Warwick, Gilb. de, iii, 277 ; Phil., ii, 52

Washers Wood (Tingrith), iii, 435

Washinglenes Gate (Wootton), iii, 329

Washingley, man. (Cranfield), iii, 276

Washington, - , ii, 257

Wassingle, John de, iil, 277 ; Will. de, iii, 276, 277

Wast, Nigel (Neil) de, i, $351 n$; ii, 324 ; iii, 6o, 260, 27I, 316, 317, 3 I 8

Wastell, John, i, 377

Water, Alice atte, iii, 92 ; Hen. atte, iii, 92 ; see also Waters

Waterbeach convent; i, 326 ; iii, 40

Water Eaton (Bucks), ii, 30 n

Water End (Eversholt), iii, 375

Waterend (Wrestlingworth), ii, 255

Watergate Farm (Battersden), iii,

Waters, Fdm. T., ii, 318 ; see also Water

Wateson, Rev. Geo., iii, 275; see also Watson

Watford (Herts), i, 358, 360

Watford, Giles de, iii, 449; Ralph, iii, 449 ; Will. de, iii, 449

Watkins, man. (Steppingley), iii, 324

Watkins (Watkin), Eliz., iii, 9x Hen iii, 325 ; Jas., iii, 9 I
Watling St., i, 37x ; ii, I, 3, I 7, 23 : iii, $350,35 \mathrm{I}, 354,390$

Watson, Frances, ii, 289 ; Rob., iii, $157 n$; Thos., iii, 226; Will. C., iii, $92 ;-$, ii, 44 ; see also Wateson

Watton Priory (Yorks), ii, 274

Watts, Hugh, ii, 269, 299 ; iii, 29 , $54,63,68,76,135,160,164,179$, I85, 208, 2I 4, 226, 233, 283, 304, 313; Will., ii, 279; iii, 382 ;

Wauberge (Waybridge, Hunts), forest, iii, 22

Waudari, Rob. de, i, $315 n$; ii, 350, 356

Waukelyn, Hen., iii, I37; Marg., iii, I 37 ; Steph., iii, 137

Waulud's Bank (Luton), i, x60, x62, $163,164,166,174,175,268,269$; ii, $8,34^{8}$

Wauton, Anne, Lady, iii, I93: Cicely, iii, I93 ; Eliz., iii, ro5: Ellen, iii, 193; Emma (de), iii, 192, x93 $n$; Joan de, $i, 357$, John, iii, 193 ; Rob., iii, 192, I93 ; Sir Thos., ii, 36 ; iii, 193; Thos. de, iii, x93; Will. of, i, 38x

Wautons (Eaton), iii, 193

Wavendon (Bucks), iii, 271, 336, $46 \mathrm{x}$

Wavendon Heath (Bucks), ii, $15 n$, 329

Wavyle (Wanwyle, Weavile), Alan de, iii, I 73 ; Hen. de, iii, 39I; John de, iii, x 73 ; Rog. de, iii, I73; Rich. de, iii, 39I; see also Wy'vell

Wayte, Agnes, iii, 218 ; Edm., iii, 218 ; Hen., ii, 329 ; Joan, ii, 329 ; John, ii, 329

Weald, Will., iii, 50 ; see also Weld

Weapons, hronze, i, 169 ; Iron Age, i, 170 ; neolithic, i, I60, 166

Weavile, see Wavyle and Wyvell

Wehbe (Webb), John, ii, 329 ; iii, 233 ; Thos., iii, 212 ; Will., iii, 233 Webster, John, iii, 184, 221 ; Will., iii, I84, 201, 221

Weddel, Thos. P., ii, 327

Wederhore, Will. de, i, 377

Wedewessen, Alice, ii, 302 ; John, ii, 302 ; Sim., ii, 302

Wedon, see Weedon

Weedon (Bucks), ii, 68

Weedon (Wedon), Isabel, iii, IIo; Ralph de, iii, 129, I33

Weedon Beck (Northants), i, $376 n$, 377

Weedon Pinkney, man., iii, 424

Wehsnade, see Whipsnade

Welbey, Rich., ii, 278

Welbourne, Rob., iii, 32

Welch, Will., iii, 449

Weld (Welde), Frances, Ladv, iii, 44I ; Mary, Lady, ii, 3or

Welfitt, S. J., ii, 195

Welitone, see Willington

Well, Geoff. atte, ii, 89 ; John atte, ii, 89 ; Rog. atte, ii, 89 ; Steph. atte, ii, 89 ; Thos. atte, ii, 89; see also Wells

Wellefed, see Wellesford

Welles, see Wells

Wellesford (Wellefed), Alice, ii, $353 n$; iii, 292

well Estate, the (Steppingley), iii, 325

Wellesworth, Rog. de, iii, 299

Wellhead Flantation (Maulden), iii, 313

Wells, i, I3, 26 ; ii, $2,5,7$; iii, 44 $74,83,94,100,114,153,157$, $228,320,349,390,402,457$

Wells (Welles), Arth. C., ii, 318 ; Edw., iii, 32; Ellen, iii, 456;
Wells (cont.)

Fran., iii, $4 x x$; Hugh de, see Lincoln, bps. of ; John, iii, 30 4II, 432 ; Marg., iii, 4II ; Rich. ii, $185 n$; iii, 328 ; Thos., iii, 53 , 456 ; - ii, I9o

Welstead, Leonard, iii, 436

Welton, Alelmus de, iii, 448, 449 Joan de, iii, I18; John de, iii I 8 ; Rog. de, iii, 118, 448 ; Will. de, iii, I I $8,44^{8}$

Weltone, par., see Willington

Wendover (Bucks), iii, 54, 372

Wendover, Rog. of, ii, 28

Weneslai, half-hund., ii, 201

Weneslai (Wennesli), Hen. de, ii, 263

Wenlock, Lds., i, 400 ; ii, 36,37 355,368 ; iii, 292 ; John, ii, 35 I $n$ $352,358,364$; iii, 299

Wenlock, Sir John, ii, 36, 305 . Marg., ii, 305; iii, 292 ; Sir Thos., ii, 372 ; Will., i, 400 ; ii, 305 35 I $n, 352,372$; iii, 292 ; fam. ii, 307

Wennesli, see Weneslai

Wensi, the chamberlain, iii, 402

Wensleydale, iii, 270

Wentworth, Henrietta M., baroness, ii, 56,58 ; iii, $44 \mathrm{I}, 445,453$; Martha, baroness, iii, $44^{I}$; Phila delphia, Lady, iii, 44I ; Lds., ii, 42 , 43 i iii, 381 ; Hen., iii, 440 ; Thos. iii, $346,440-I, 445$

Wentworth, Anne, iii, 266; Sir Giles, iii, 283; Jane, iii, 346 , 445: John, iii, 266 ; Marg., iii, 283 ; Maria, iii, 445; Sir Thos. iii, 445; see also Strafford, Ld. Will., iii, 44I; fam., ii, 56 ; iii, 283

Wernher, Sir Julius, ii, 349, 353, 355 Weseham, Hamon de, iii, $284 n$. Rog. de, iii, $284 n$

Wesley, John, i, 344-6

Wesleyans, ii, $2 \times 4,237,24^{x-2}, 246$ $255,265,270,299,303,312,320$ $333,335,344,374,387$; iii, $3 \mathbf{x}$ $59,63,76,117,128,136,139$ $144,157,168,171, x 75,181,190$ $234,268,275,284,296,297,305$ $307,308,313,314,316,320,326$ $328,338,345,367,383,389,4$ I 4 $415,418,424,432,45^{8}$

Wessex, kingdom of, $i, 309 ;$ ii, 20

Wessex, Godwin, earl of, ii, 20

West, Edm., iii, 286 ; Fran., iii, 348, 349,386 ; Ida, iii, 380 ; Marmaduke, iii, 18 ; Nich., iii, 286, 442 Rich., ii, 289; Thos., iii, 380 Walt., ii, $334 n$; Will., iii, 286

Westbury (Bucks), ch., i, 357

Westbury, Agnes of, i, 357; Will. iii, I 50 n

Westcotes (West Cotton), man (Wilshampstead), ii, 324 ; iii, 326

West End (Cranfield), iii, 275

West End (Little Staughton), iii, 165

West End (Silsoe), ii, 326, 338

West End (Stagsden), iii, 96

Westende, man. (Gt. Barford), see Creakers, man.

Westterdale, Nich., iii, 247

West Field (Westoning), iii, 455

Westhey, Thos. de, ii, $346 n$

Westhey and Faldo, man. (Higham Gobion), ii, 346

Westminster Abbey, i, 312 ; ii, 153 , $21 \mathrm{I}, 235,286,34 \mathrm{I}$; iii, I2, 260, 401; John, abbot of, ii, 2 I2 $n$

Westminster Pond (Thurleigh), i, 289

Westmoor Field (Radwell), iii, 305 


\section{A HISTORY OF BEDFORDSHIRE}

Westmorland, price of wool in, ii, 91

Westmorland, Ralph, earl of, iii, 13 ; Ld., iii, 94

Westnynge, see Westoning

Weston, Jeremy, iii, 277 ; John, ii, 330: Rich. iii, 116, 272, 277, 396 ; Sir Will, iii, 142; Will. de, ii, 227 ; iii, 157

Westoning, i, $315 n$; ii, 44,72 , $81,86 n, 88,92, I_{4}, 185$; iii I 79, 336, 45I-5 ; adv., iii, 454 ; chant., ii, 285, 299; iii, 453; char., iii, 455; ch., i, $314 n$ $325 n, 357$; iii, 453 ; dean of, i, 347 ; ind., iii, 451 ; inns, iii, $45 \mathrm{I}$ mans., i, 303, 370 ; ii, 80,226 ; iii, $443,45 \mathrm{I}-3$; mkts. and fairs, ii, 88 ; iii, $452-3$; mills, iii, 453 ; Nonconf., iii, 45 I ; place-names, iii, 45I i prehist. rem., iii, 45I ; sch., ii, 185 ; vicarage, i, $316 n$

Westoning Wood End, iii, 45 I

Weston Tregoz, see Westoning

West Wood (Knotting), iii, I 39

Westwood (Lidlington), iii, 305

Wetaway, Will., iii, 291

Wettele, see Wortele

Weylondon, Agnes de, iii, 452 ; John de, iii, 452 ; Juliana de, iii, 452

Whalley, Edw., iii, $173 n$; Rich., iii, $173 n$; Col., ii, 50, 52; iii, $45^{8}$

Wharley End (Cranfield), iii, 276

Wharton, Lady Anne, ii, 340 ; Lady Dorothy, iii, 236; Phil., Ld., iii, 236

Wharton, Geo., ii, 378

Whatwell Mill (Shillington), ii, 297

Wheathampstead (Herts), prehist. rem., i, 150

Wheathampstead, John of, i, 351, 352 ; ii, $342,368,369$; iii, $148 n$, $44^{8}$

Wheatley, Eliz., iii, 184

Wheeler, Will., ii, 329,382 ; iii, 333

Whetham, Thos., iii, 192, I 97

Whetharnstede, see Wheathampstead

Whikey, man. (Little Staughton), iii, 166

Whiperley, man. (Luton), ii, 350, $35^{8}, 360$

Whipsnade, i, 3 I $5 n, 318$; ii, II $3 n$, I14, I85; iii, $336,337,407 n$, 426, 430, 449, 455-7; adv., iii, 457: chap., i, 318; char., iii, 457; ch., i, 315n; iii, 456; Hill Farm, iii, 455 ; mans., iii, 371, 455: Nonconf., iii, 455: place-names, iii, 455 ; sch., ii, 185

Whipsnade, Ad. de, iii, 455, 457; Alice de, iii, 456; Christina de, iii, 456 ; Gilb. de, iii, 456 ; Joan de, iii, 456; Margery de, iii, 456 : Matilda de, iii, 456 ; Nich. de, iii, 456 ; Phil. de, iii, 456 ; Rich. de, iii, 455 ; Will. de, iii, 455

Whipsnade Heath, iii, 448,455

Whipsnade Wood, iii, 369,455

Whitaker (Whitacre, Whiteacre Whytacre), Bennet (Benedicta) of, i, 360 ; Hen., ii, I62 ; John, ii, 4 I ; P. A. O., ii, I89: Rich., iii, 129

Whitbread, Emma, iii, 41; John, iii, 259 ; Sam., ii, 64, 65, 66, I76, 238, 299; iii, 9, I5, 20, 21, 32, $41,140,206,226,234,235,236$, $237,238,253,254,255,256,257$, 258, 259, 26r, 28r, 283,327
Lt.-Col. Sam., ii, 70 ; Sam. C. iii, 236 ; Sam. H., ii, 66 ; iii, 234 :
Whitbread (cont.)

Steph., iii, $23^{8}$; S. C., ii, 189 ; Will., ii, 334 ; iii, 259 ; Will. H., ii, 65,194 ; iii, I5, 235,253 ; Miss, iii, 234; 一, ii, 132, 197 ; iii, 139; fam., ii, 108, $130,33^{8}$; iii, 258

Whitby, fam., see Whytbie

Whitchurch (Bucks), i, 370 ; man., iii, 3 Io

Whitchurch, Bald. of, iii, 427 ; John A., iii, I8I ; 一, ii, 190

White (Whyte), Ágnes, iii, 225 ; Bertram le, iii, 46: Fran., see Norwich, bp. of ; John, ii, 359; iii, 236, 366: Sir Thos., ii, I58, 159: Thos., iii, 225; Will., iii, 225 Whiteacre, see Whitaker

Whitehead, Eliz., ii, 289; Hen., ii, 337 : Thos., ii, I51, I82 $n$; iii, 394: W., ii, I95, 337 : Mrs,, iii, I60, 162

Whiteman, Eliz., iii, 238

Whitemore, see Whitmore

White Way (Rom. rd.), ii,

Whitfield, Ralph, iii, 201

Whiting and lime manufacture, ii, I I 7 ; iii, 350,363

Whiting (Whyting), Will., ii, 89

Whitlocke (Whitlock, Whitloke), Bulstrode, ii, $54 n$; iii, 8 ; John, iii, 414 ; J., iii, 194; Kath., iii, 4II ; Rich., iii, 4I I

Whitmore (Whitemore, Whittmore), Geo., iii, I75 $n$; Tom, ii, 189 ; Will., iii, 331, 374

Whitnoe (Whitnow) Grange (Woburn), iii, $459 n, 461$

Whittington, Jacob J., ii, 256, 257

Whittlebury (Northants), iii, 462

Whittmore, see Whitmore

Whitwick, man. (Thurleigh), iii, Io6

Whitwick Green Farm (Thurleigh), iii, 104

Whitworth, John, iii, 285 ; Kath. iii, 285 ; Miles, iii, 277 ; Will., iii, 92

Whytacre, see Whitaker

Whytbie, Oliver, i, 337 ; Thos., i, 336

Whyte, see White

Whyting, see Whiting

Wiboldestune, see Wyboston

Wichalse, Arth., iii, 274, 316, 319, 323

Wiche, Matth. atte, iii, 98

Wick End (Stagsden), iij, 96

Wickend Spinney (Stagsden), iii, 96

Wicker-work ind., ii, 124-5; iii, 35,76

Wickey Farm (Little Staughton), iii, 166

Widville, see Woodville

Wiffen, Benj. B., iii, 338, 458 ; Jer., iii, $45^{8}$

Wigain (Wigan), Hen., iii, 206 : Hugh, iii, 206; Ralph, iii, 206 ; Rog., iii, 206 ; Walt., iii, 240 ; Will., iii, 206

Wigg, fam., iii, 407

Wiggons, man. (Cople), iii, 240

Wightman, Will., ii, 257

Wigmore, hon. of, ii, 328

Wigram, F. S., ii, 189

Wigstaffe, see Wagstaffe

Wilbarston (Northants), ch., i, 357

Wilbon, Will., iii, 293

Wilbraham (Cambs), ch., ii, 217

Wilbraham, Little (Cambs), A. S. rem., i, I $79 n$

Wilde, see Wylde

Wildeboef, see Oyldeboef

Wilden, ii, $113,145,147$; iii, I26, $168,180,182 n, 214,223-6$;
Wilden (cont.)

adv., iii, 226 ; char., iii, 226 ch., iii, 225 ; mans., iii, 2 II, 223 place-names, iii, 223 ; schs., ii, 185 ; iii, 226

Wildman, Jas., ii, 316

Wilford, Christiana, iii, $443 n$ Thos, iii, $443 n$

Wilkes, Charlotte, iii, 416; Edw., iii, 4II, 415; Joan, iii, 411 John, iii, 4I5, 45I : Luke, iii, 4 II ; Matth., iii, 4I5 ; fam., iii, 4 II

Willaume, see William

Willes (Willis), Edw., ii, 181 , 310 313 ; Sir Fran., ii, 210; Will., ii, IOI, 166,167

Willesdon, Barth., ii, 3 Io

Willett, H., ii, 20o; Willmer M., iii, 189

Willey, hund. of, ii, 115 ; iii, 34-58

Willey (Willy, Wilye), Bern., iii 310, 313: Brown, ii, I 70; Hugh de, iii, 34, II3 ; Rog. de, iii, 34 113,147

William, iii, 57, 302: the chamberlain, $i, 311,314,3$ I $5 n, 344$ the clerk, i, $319 n$; iii, 404 the steward, iii, 302

William (Willaume), Chas. D., iii, 9I, 436, 444 ; Dav., iii, 436, 438 , 444 : Dionisia, iii, 105; Edw., iii, 436 ; John, iii, 436

Williams, Dav., ii, 173 ; Edw. G. iii, 383 ; Rev. Hamilton J., iii, 304 ; John, archbp., see York archbps. of ; John, i, 368 ; ii, 256 ; iii, 461 ; Rich., ii, 239 : Will, iii, 285; - (Ld. Keeper) iii, $45^{8}$

Williamson, Anne, iii, I19; Chas. iii, 119; Rev. Edm., iii, 398; Edm., iii, 396, 398; Rev. Edm. R., iii, 298; John, ii, 207, 301 ; Ralph, iii, I 22; Sara, ii, 207 , 301 : Talbot, iii, 396; Mrs., iii, 298, 299

Willington, i, 284, 324, 392; ii 60,96 , 101, 116, 138, 339: iii, 227, 262-6; adv., iji, 266; ch., i, $134,315 n, 380$; iii, 262 ? earthworks, i, 280, 282 ; Manor Farm, iii, 262; mans., iii, 12, $184,203,204,262$; mill, $i, 392$;

Rom. rem., ii, 15 ; iii, 262 ; sch. ii, 185 ; vicarage, $i, 316 n$

Willington, man. (Cople), iii, 240

Willington, Eleanor, iii, 298; Hen. de, iii, 298 ; Will. of, i, 399

Willis, see Willes

Willoughby, Eliz., ii, 211 ; Mary, iii, 335, 435 ; Rob. de, ii, 21

Willoughby de Broke, Ld., ii, 203 n Willoughby de Eresby, Ld., iii, 13 , 14

Willow Close (Ampthill), iii, 274

Willowes, Mrs., i, $335 n$

Willy, see Willey

Wilshamstead (Wilsamstead), i, $352-4,356$; ii, I1 5, 293, 34I, 342 ; iii, 267, 279, 281, 284, 325-8; adv., iii, 328 ; chant., i, 326 ; char., iii, 328 ; ch., i, 3 I $5 n$, 326,357 ; iii, 326 ; farms, iii, 274,326 ; mans., i, 313 ; iii, 326 ; Nonconf., iii, 326, 328 ; sch., ii, I 85 ; iii, 328

Wilshamstead Wood, iii, 325

Wilshire (Wilshere), John, ii, 252 ;

J., ii, I 8 I $n, 3$ I 3 ;'Thos., ii, 313

Wilson, Eliz., ii, 268; Felix, ii, 278 , 310, 323; Jas., ii, 285 : Tim., ii, 268

Wilstead (Cardington), iii, 238

Wiltone, see Willington 


\section{INDEX}

Wiltshire, Edm. earl of, iii, r25 ; John earl of, iii, 106

Wilye, see Willey

Wimberville, Rog. de, iii, $405 n$

Wimington (Wimmentone), see Wymington

Wimund, iii, $\mathrm{II}_{4}$

Wimund (Wymund), Pet., iii, 30

Winch (Wynche), Eliz., ii, 340, 375: Sir Humph., ii, $60 n, 227$, 229, 24I, $340 n$; iii, 8 ; Humph. ii, 55, 227, 235; John, ii, 235; Sir Onslow, ii, 201, 202, 227, 228, 340,34 I $n$; iii, 35,227 ; Rich. atte, i, 400 ; fam., ii, 60

Winchelsea, archbp., i, 375

Winchendon, Lower (Bucks), iii, $3^{1} 7$

Winchester, archd. of, i, 327 ; Pet. des Roches, bp. of, ii, 29 ; iii, 439 ; Rich. Fox, bp. of, iii, $\mathrm{r}_{3} 8$

Winchester, earls of, ii, 28 ; Rog., iii, ror, 230 ; Saher, iii, 230

Windmill Field (Potton), ii, 24I

Windmill Hill (Eaton Socon), iii, 189

Windmill Hill (Leighton Buzzard), iii, 404

Windmill Hill (Silsoe), ii, 326

Windmill manufacture, ii, 127

Windsor, St. George's coll. ch., i, $376 n, 404$; ii, 218 ; iii, 366 , $401,416,429$

Windsor, Maurice de, iii, 229

Winessamestede, see Wilshamstead

Winfeud, Winfield, see Wingfield

Wingate (Wyngate), Anne, iii, 4 ro ;

Arth., ii, 362 ; iii, 38r ; Button, iii, 33r, 335; Edm., ii, $54 n$; iii, 331, 335, 38r ; Edw., ii, $354,355,362,374$; iii, 393 ; Eliz., ii, 363 ; iii, 335; Sir Fran., ii, $362,3^{63} n$; iii, $367,381,382$; Geo., ii, 362,363 ; iii, 367,410 ; John, ii, 43, 362, 363, 367, 381 ; Kath., i, 356; Mary, ii, 354, 363 ; Ralph, ii, 286 ; iii, 366 ; Rob., iii, 410; 一, Lady, iii, $3^{81}$; fam., iii,

Wingfield, iii, 345, 347 ; man., iii,

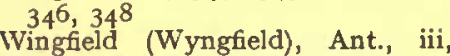
270 ; Chas., iii, 172 ; Edw., iii, 37 ; Sir Jas., iii, 168, 172 ; Sir Rich., iii, 1 72, 396 ; Rich., iii, 82, 172 , 272: Thos., iii, I 72 ; Trevor, iii, $40,45,55$; Misses, iii, 268

Winkbourn (Notts), i, 394

Winslow, Will. of, i, 353

Wintremelc, Alric, iii, 206

Wintroe Corner (Ridgmont), iii, 321 Wiscard, Hen., ii, 329 ; Rich., ii, 378,379 ; iii, 260 ; Will., ii, 329

Wisdom, Geoff., ii, $215 n$; Mabel, ii, $215 n$

Wiseman, Anne, ii, $33^{8}$

Witchcraft, ii, roo; iii, 402

Witham, riv, i, 18 ?

Witintona, Hen. of, ii, $293 n$

Wits End (Eversholt), iii, 375

Wittenham, Long (Berks), A. S. rem., i, I $78,183,188$

Witton, Rev. - , iii, 435

Wixamtree, hund., ii, 18, I13 $n, 116$, 242 ; iii, 227,267

Wlui, bp., iii, 314

Wlward, see Wulfweard

Woad, ii, 130 ; iii, 44

Woburn, i, 362, $363 n, 370,374$ ii, $17,47,52,67,102,1_{14}, 1_{32}$, I35, $171,197,377 ;$ iii, 4,336 , $343,353,382,383,400,457-62$ adv., iii, 462 ; agric., ii, 133,134 $\mathrm{r}_{3} 8, \mathrm{r}_{4} \mathrm{O}, \mathrm{r}_{4} \mathrm{r}$; iii, 394 ; chap., $\mathrm{i}_{\text {, }}$ 318, 331. $368,369,370$; iii, 422 ;

\section{Woburn (cont)}

char., iii, 462 ; Chas. I. at, ii, 50, 51 ; iii, 458 ; chs., iii, 46r ; crown property, iii, $27 \mathrm{I}$; farms, iii, $45^{8}$; fires in, iii, $45^{8}$; hosp., iii, 457 ; ind., iii, 458 ; inns, ii, 99 ; iii, 458 ; mans., i, $366,367,370$; iii, 459; mkts. and fairs, ii, 88: Nonconf., iii, $45^{8}$; place-names, iii, 459; racing, ii, I9I; Rom. rem., ii, 15; schs., ii, 185; iii, 462 ; sheep-shearing, iii, 459

Woburn, Walt. de, iii, $449,450,456$ Woburn Abbey (anct.), i, 318,330 , $331,349,350,35 \mathrm{I}, 366-70,37 \mathrm{I}$, $372 n, 402$; ii, $40,346,37^{6}$; iii, $24,324,347,362,370,375,376$, $377,379,380,385,387,397$, $4 \mathrm{r} 8,4^{\mathrm{r} 9}, 422,44^{2}, 443,44^{8}, 453$, $458,459,46 \mathrm{r}, 462$; abbots, $i$, $318,370,374,402$; mill, $i, 366$

Woburn Abbey and Park, ii, 47, $69, \mathrm{r}_{7}, \mathrm{r} 33, \mathrm{r} 46$; iii, 320,338 , $375,394,417,421,458,459$; royal visits, iii, $458,459,460$

Woburn Hills, ii, 7 r, 129

Woburn Sands (Aspley Guise), ii, I17, I29; iii, $275,336,33^{8}$; ch., iii, 338 ; fuller's earth, ii, I I 7

Wode, see Wood

Wodehouse, Rob., ii, 287

Wodemancote, Emma de, ii, 379 ; Rob, de, ii, 379

Wodhull, see Odell and Wahull

Wolaston, see Wollaston

Wolf, John le, iii, 5I, 60

Wolfmar, see Ulmar

Wollascott (Woolascote), Joan, ii, 227-52; Will., ii, 227, 228, 252

Wollaston (Northants), ii, rgo; iii, 209

Wollaston (Wolaston), Sir John de, iii, 90; Marg., iii, 9o ; Sim. de, iii, $49,49 n$

Wolley, Rich., ii, 364 ; Rob., ii, 364

Wolstenholme, Sir John, iii, $73 n$; Lady Temperance, iii, 68, 76 ; Lady, iii, 66, 74

Wolston, see Wollaston

Wolverton man. (Bucks), i, 39r, 393

Woobourn, see Woburn

Wood (Wode), Marg., iii, 456; Nigel atte, iii, 52 ; Oliver, iii, 456 ; Rich., ii, 266; Thos., iii, IIo; Will. (atte), iii, 245, 450

Woodburn, Mrs. Pen, ii, 289

Woodcock, Edw., iii, 23

Wooderoft (Luton), ii, 354, 374 man., ii, $350,354,356-8,362$, 364,366 ; iii, 405,422 ; mill, ii,

Wood Croft (Ravensden), iii, 210

Woodcroft (Stevington), iii, roo

Wood End (Bolnhurst), iii, 124

Woodend, man. (Cople), iii, 238, 239

Wood End (Cranfield), iii, 276

Woodend (Harlington), iii, $3^{80}, 442$ Wood End (Marston Moretaine), iii, 308

Wood End (Ravensden), iii, 209, 21

Woodend Plant (Sharnbrook), iii, 89

Woodfield (Luton), ii, 36I

Woodford, Hen. of, ii, $38 \mathrm{r}$; Will. of, ii, $38 \mathrm{r}$

Woodhall, man. (Meppershall), ii, 290

Woodhall Farm (Meppershall), ii, 288

Woodhill, see Odell and Wahull

Woodhull, see Odell and Wahull

Wooding, Rob., iii, $5^{8}$
Woodleys (Melchbourne), iii, 142

Woodmanley man., iii, 456

Woodmer End (Shillington), ii, 294

Woodside (Caddington), ii, $314 n$, 3rg

Woodstock, Mary of, iii, 403

Woodville (Widville, Wydville, Wydvile), John, iii, 36,386 ; Kath., iii, 36, $3^{86}$; Sir Rich., iii, Ir 8,386 ; Thos., iii, $36,48,424$

Woodward, Edm., iii, 295; Ellen iii, 346 ; Mary, iii, 425 ; Rob., iii, 425; Tristram, iii, 346,440 Walt., i, $356 n$; Dr., iii, 425 一, ii, 166

Woodweston, John of, i, 399 ; Will of, i, 399

Woodyard Close (Luton), ii, $36 \mathbf{r}$

Wooldridge, Thos., iii, 25

Wool ind., ii, 91, 1or, 363

Woolsey Wood (Turvey), iii, II 4

Woolward, Rev. S. A., i, I 72

Wootton, i, 174, 338; ii, 44, 115 I $87,213,245,292,303,312,324$ 335 ; iii, 39, I32, 147, I 56, 233 $267,278,297,315,328-35,348$ $378,398,4$ I 4, 435, 454 ; adv., iii 334 ; chants., iii, 335 ; char., iii, 335; ch., i, 315n, $325 n, 333$ $334 n, 379,380 ;$ iii, 333 ; ind. iii, 329 ; man., iii, $13,89,329$, $330,33^{2}$; sch., ii, 185 ; vicarage, i, 3 I 7

Wootton (Wotton), Alex. de, iii, 132; Constance de, iii, 309 Isabella de, iii, $35^{8} n$; John de iii, $358 n$; Rich. de, iii, $\mathrm{r}_{32}$ Rob. de, iii, 309, 3r 3 ; Walt. of i, 324 ; iii, 313

Woottonbourne (Wootton), iii, 33I

Wootton Green (Wootton), iii, 329

Wootton Hoo, man. (Wootton), iii 329,330

Wootton House (Wootton), iii 328

Wootton Pilling (Wootton), iii, 332

Wootton Shelton, man. (Wootton) iii, 333

Worcester, Walt. Cantilupe, bp. of, ii, 32

Worcester, Eliz., ctss. of, ii, 354 earls of, iii, I73; John, ii, 354 iii, 329

Workhouse End (Renhold), iii, 214 Workhouses, ii, ro 4

Wormleghton, Wormleighton, par. see Wrestlingworth

Wormleighton, Rob. Spencer, Ld. ii, 220

Worship, Joan, iii, 403; John, iii, 403

Wortele (Wettele), Nich. de, iii, 145

Wortham, Hale, iii, 428

Worthing, Thos., ii, 326

Worthington, Will., ii. 310

Wotton, see Wootton

Woughton, see Wauton

Wrastlingewurde, see Wrestlingworth

Wray, Sir Chris., iii, $38 \mathrm{I}$

Wresle, John de, iii, 247

Wrest, man. (Silsoe), ii, $184 n, 283$ $290,305,326,33 r, 334,336,345$; iii, 65,82 ; ch., ii, 333 ; mill, ii 331 ; vicarage, ii, 333

Wrestlingworth (Wrestlingforth), i, 345 ; ii, 72 , $\operatorname{rr} 3,201,230,233$ $243,255-9$; adv., ii, 257,258 char., i1, 258, 259; ch., i, $315 n$ ii, 257: mans., ii, 255: mills, ii 257; Nonconf., ii, 258 ; placenames, ii, 255 ; sch., ii, 185

Wrestmead (Flitton), ii, 330

Wrest Park, ii, 36, 44, 61, 145, 197 , $308,325,331,333$; iii, 45 I 


\section{A HISTORY OF BEDFORDSHIRE}

Wright, Geo., iii, 409 ; Sir Hen., iii, I 40; John, ii, 214; iii, 129 Dr. Jos., ii, $18 n$; Lawr., iii, 95 ; Sir Nathan, iii, 105; R. S., ii, I74, I75; Will., iii, 409 ; - , ii, 40

Wriothesley, Ld., ii, 299 ; Thos., Ld., ii, $57 n$; iii, 203

Wroxhill (Marston Moretaine), see Roxhill

Wroxill, Will,, i, 398

Wulfhere, king of Mercia, i, 309

Wulfig (Wulfwig), see Dorchester, bp. of

Wulfmar, iii, 190

Wulfweard (Wlward), 'Levet,' iii, 439

Wutton, see Wootton

Wyatt (Wyat), Geo., iii, I29; Sir Hen., ii, 359 ; iii, 396 ; Hen., iii, 272 ; Jas., i, I76; ii, 273 ; Rev. Paul, iii, 2 I $n$; Sam., iii, I25, r46; Thos., ii, I6I; iii, I64; -, ii, 326

Wyboston, i, 308, 312 ; ii, 324 ; iii, 189-9o, 191, 193, 196, 198, 199; chant., i, $329 n$; iii, 20I ; chap., $i, 326 n, 329 n$; man.-house, i, 305-6 ; mans., iii, 191, 196, 197. 199

Wyconibe, West (Bucks), ii, $45 n$

Wye, Thos., ii, 380

Wygod, Hugh, iii, 225

Wygood (Wilden), iii, 225

Wylde (Wilde, Wild) Dorothy, Lady, iii, 216,293 ; Edm., ii, 180, $182 n$; iii, 296 ; Sir Edm., iii, 216, 293; Eliz., jii, 48; Sir John, iii, 300; Thos., iii, 48, I79; Will. iii, 7 ; Mrs., iii, 179

Wylibesende, see Whipsnade

Wyllyngton, Wyliton, see Willington Wylter, see Walter

Wymington, ii, $7 \mathrm{I}$, II $5, \mathrm{I} 85$; iii, $34,70,80,87$, I $17-22$, I 78 ; adv., iii, I22; bronze impl, i, 169, I74 ; char., iii, 122; ch., iii, I 20 ; ind., iii, II 7 ; man., iii, II 7 ; man.-house, iii, II 7 ; mill, iii,
Wymington (cont.)

II9; Nonconf., iii, II 7 ; sch., ii, 185

Wymington, John, i, 383 ; Rog. of, i, 382, 384; Will. de, iii, 196

Wymondley, Little (Herts), i, 294

Wymund, see Wimund

Wynche, see Winch

Wyndham, Thos., ii, 27r, 275

Wyngate, see Wingate

Wyngfield, see Wingfield

Wynn (Wynne), Hen., iii, 259 John, ii, 246 ; iii, 259

Wynum, Walt., iii, 322

Wyrell, Joan, i, 36r

Wyscard, see Wiscard

Wytham, Hercules, iii, 432

Wythes, Fran., iii, 2 I0 Fran. A. W., iii, 2 ro

Wythes Close (Stotfold), ii, 304

Wyttelbury, Rob., iii, 126

Wyvell, Agnes, ii, $352 n$; Nich., ii, $352 n$; Will., ii, 352 ; see also Wavyle

Yarborough, Chas. A. Pelham, Ld. ii, 231

Yarborough, Geo. C., ii, 245

Yardley, John, ii, 95

Yarlswood (Thurleigh), i, 308

Yarrow's charity (Sandy), ii, 246

Yarway, Rob., iii, 298, 331, 335

Thos., iii, 331, 335 ; - , ii, 44

Yeaveley preceptory(Derb.),ii, $276 n$ Yelden, i, 290; ii, 7I, II 5 ; iii, $36 n$ 123, I 34, I 36, I60, I68, I 75-9, 330 ; adv., iii, I79; char., ii, $185 n$; iii, I79; ch., iii, I77; man., iii, 54, I75, I79; Nonconf., iii, I75; sch., ii, 185

Yelden Castle, iii, I75; earth works, i, 289, 291 ; Rom. rem. ii, I, I 5 ; iii, I 75

Yelnoe Wood (Odell), iii, 69

Yelverton, Sir Chris., iii, 82, I 19 : Sir Hen., iii, zor ; Hen., iii, 82, I 9 ; Sybil, ii, 82, Ir 9
Yielden, see Yelden

Ympie, see Impey

Yon, see Gravenhurst, Lower

York, archbps. of, Murdak, i, 390 n Oskytel, i, 3II: Rotherham, ii 157,2 I9, 295, 352 ; iii, 292, 299 ; Thurstan, i, 361; Williams, i, $339 n$; iii, 264

York, Anne, ctss. of, ii, 301,340 ; dks. of, ii, $57,67,192,301,340$ iii, 4, 404 ; Rich., ii, 301, $34^{\circ}$ : iii, 13

York, Nich. of, iii, 277

Yorke, Amabella, ii, 327 ; Phil., ii, 61,380

Yorkshire Farm (Ravensden), iii, 210

Young, Arth., ii, 96, 97, I19, 130 I3I, I33, I34, I 40 ; Barth., i, $326 n$ Freeman, iii, 432 : Gabriel, iii, 125; Mark, iii, 264

Younges, man. (Westoning), iii, 453

Yprès, John of, i, $3^{82}$

Y thingaford, iii, 4 or

Zoology, i, 69-1 43

Zouche, Lds., iii, 394 ; John, ii, 316:'Will., iii, 37r

Zouche, Alan la, ii, 328 ; Alice, la, iii, $440 n$; Ellen la, ii, 328 . Eudo de la, i, 373 ; ii, 225, 282 315 ; iii, II $8,370,371,392,449$ : Geo. la, iii, 452 ; Ivo de la, ii, 336 ; Joan, i, 360,361 ; ii, 336 ; iii, 449,452 ; John de la, ii, $225 n$, 282 ; iii, I44, 37I, 452; Mary la, ii, 243 ; Mand la, ii, 328 ; iii, 176 , 243: Milicent, ii, 225, 282, 373 . iii, $37^{\circ}$; Rich. de la, ii, 282 ; Rog. la, ii, 328 ; Thos., ii, 243 ; Sir Will. la, iii, 360 ; Will. de la, ii, 282,316 ; iii, $176,243,370$, $37 \mathrm{I}, 372,39 \mathrm{I}, 394,440 \mathrm{n}, 449$, 452 ; fam., ii, 282,3 I 5 ; iii, II 8

Zourches, man. (Caddington), ii, 3 I 5 , 3 I 6

Zouches Farm (Caddington), ii, 3 I 6 318 


\section{CORRIGENDA AND ADDENDA}

Vol. I, page 4I, line 9, for 'Charad' read 'Chara'

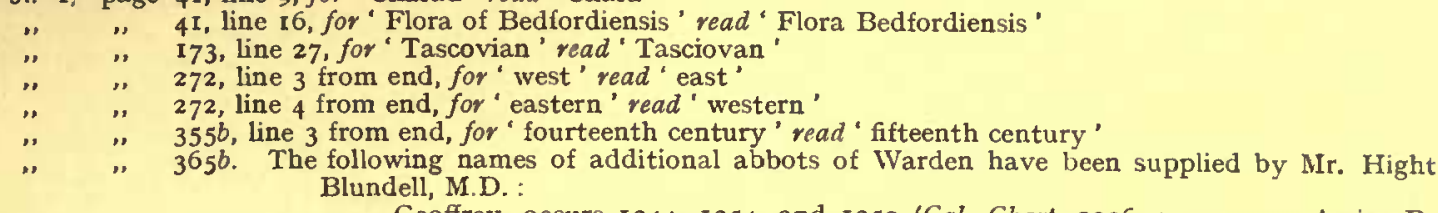
Blundell, M.D.

Geoffrey, occurs I244, I254, and 1259 (Cal. Chart. 1226-57, p. 279; Assize R. no. $23, \mathrm{~m} .72$; Feet of F. Beds. file 25, no. 67)

Robert of Harrold, occurs 1272-5 (Assize R. no. 1 247)

John de Dallinger, occurs 1287 and 1290 (Assize R. no. 1276, m. 20 ; Cal. Pat. I $28 \mathrm{I}-92$, p. $37 \mathrm{I}$ )

Thomas, occurs i 318 (Cal. Pat. I $317-2$ I, p. 275)
William, occurs I 406 (Cal. Pat. 1 $405-8$, p. 236)

393a. The following names of additional priors of Chicksand have been supplied by Mr. Hight Blundell, M.D.

Hugh de Ledenham, occurs I 242-6 (Feet of F. Beds. file 20, no. 7)

Alan, occurs 1272 and 1284 (Assize R. no. 1223, m. 6 ; ibid. Northants, no. 620, 622)

Walter, occurs I 292 (Assize R. no. $1298, \mathrm{~m} .80 \mathrm{~d}$.)

Simon, occurs I 330 (Assize R. Northants, no. 633)

Roger, occurs as predecessor of John Lechworth (Assize R. Beds. no. 32)

John Lechworth, occurs I 355 (Assize R. Beds. no. 32)

William, occurs I 426-8 (Assize R. no. I 539)

Richard Spencer, occurs I 503-4 (De Banco R. Hil, I 8 \& I9 Hen. VII)

" "403. The following names of masters of Northill College have been supplied by Mr. Flight Blundell, M.D.

Richard Hethe, occurs I 422-40 (Ct. R. [Gen. Ser.], portf. I 53, no. 34)

Thomas Greneley, occurs I 459-6I (ibid.)

Thomas Taylard, occurs 1477 and 1478 (ibid.)

William Porter, occurs I 494 (ibid. no. 35)

Thomas Randolfe, occurs I 494 and I 498 (ibid.)

Andrew Benstode, occurs I 499 and I 505 (ibid.)

John Underhill, occurs 15- (Dugdale, Mon. vi, I 399)

Vol. II, page xvii, line 5, for 'Austen ' read 'Austin'

57 , note 406 , line 2 , for 'south ' read ' north'

I 45, line 40, for "Beckening ' read " Beckering

"I 149 , line 15 , for ' $£ 30,000$ ' read ' $£ 13,000$ '

"I 176, line 34 , for ' and the buildings completed 'read' and completed "

" 235a, line 43, for 'Knights Hospitallers' read " Innights Templars' (cf. Assize R. no. 4, m. rg)

" $243 a$, line I 3 , for ' Henry III' read ' Henry II

$25 \mathrm{I} a$, line $2 \mathrm{I}$ from end, for "Stewart 'read' Stuart"

$305 a$, line 14 , for "one knight's fee" read "half a knight's fee"

$326 b$, lines 29,41 , for 'Lee in Podington' read 'Lee in Thurleigh'

$384 b$, line 7 , for 'Rodenham ' read 'Bodenham'

" $384 b$, linc 33, for 'passed to Isabel's son by her hirst marriage, Richard de Clare, Earl of Gloucester,' read 'passed to Gilbert de Clare, grandson of Isabel, her son Richard having died in $1262^{\text {' }}$

Vol.' III, page 2ob, line 5, for 'Sanbridge' read' Sawbridge'

$37 a$, line 30 , for 'Butler' read 'Boteler"

$46 b$, line 9 from end, for 'Osiel 'read 'Osiet'

I Ioa, line 3, for 'Easton Mandit' read 'Easton Maudit'

$\mathrm{I} 8 \mathrm{r} b$, line 35 , for 'Tetband 'read 'Tetbaud'

2 I $b$, line 5 from end, for 'Grymband ' read 'Grymbaud "

$220 b$, line 2, for 'Grymband' read ' Grymbaud

258b, line 15, for 'Ordin' read 'Ordui

295 , note 163 , delete 'She later married Walter de Teyes'

298b, line 16 , for 'May ' read 'Mary'

335 , line 4 , for 'Britton' read 'Button'

$338 a$, line I, for 'Aspslea' read 'Aepslea'

$338 a$, "for 'Aspleia' read 'Aspeleia "

$338 a$, line 6 from end, for ' 1690 ' read 'I 7 I I'

$339 a$, line 2, for "Valery' read "St. Valery"

$342 a$, line 2, for "Wishaw' read "Wysham "

$342 b$, line 14 , for 'Booth' read 'Ansell'

$343 \mathrm{~b}$, line 3 , delete ' in labour in connexion therewith '

345 , line 24 from end, for "Guthruin' read "Guthrum

$385 a$, line 17 , for' Hamstead' read ' Flamstead '

", 390, note 8, for "Buckwood Stables' read 'Buckwood Stubbs' 


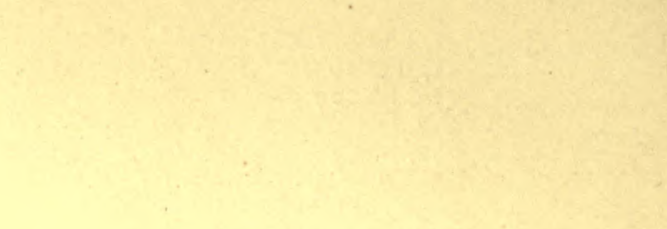




\section{SUBSCRIBERS TO THE VICTORIA COUNTY HISTORY OF BEDFORD}

\section{HIS MAJESTY THE KING}

C. Alington, Esq. W. H. Allen, Esq., J.P. A. R. Alston, Esq.

Capt. F. Alston

The Rt.Hon.The LordAmpthill,G.C.I.E.,G.C.S.I. The Rt. Hon. The Lord Annaly, K.C.V.O. His Grace The Duke of Argyll, K.G., K.T. William Austin, Esq.

C. B. Balfour, Esq., J.P., D.I.

Mrs. Barclay

His Grace The Duke of Bedford, K.G., F.R.S. The Rt. Hon. The Lord St. John of Bletsoe, D.L. Hight Blundell, Esq., M.D.

J. Hight Blundell, Esq.

Rev. C. Bromley, M.A.

Miss Brooks

A. C. Buckmaster, Esq., LL.B.

Mrs. Eleanor Carroll

The Rt. Hon. The Earl of Carysfort, K.P.

Major E. F. Clayton

E. C. Coleman, Esq.

Francis Crawley, Esq., J.P.

Miss Crewdson

William Deane, Esq., J.P.

J. Griffith Deardon, Esq.

The Rt. Hon. The Earl of Derby, G.C.V.O.

Col. C. Villiers S. Downes

Sir Robert Edgecumbe, LL.D., D.L.

Charles Emmerton, Esq.

William Farrer, Esq., D.Litt.

Miss Harriet Fitzpatrick

The Rt. Hon. The Lord Foley, D.L.

Edward Snow Fordham, Esq., M.A., LI.M.

G. Herbert Fowler, Esq., B.A., Ph.D.

C. J. Fox, Esq., M.R.C.S.

Maurice G. C. Glyn, Esq., J.P.

O. E. D'Avigdor Goldsmid, Esq., D.L.

Sir Laurence Gomme, F.S.A.

H. E. Gregory, Esq.

The Right Hon. The Viscount Hambleden

John Hamson, Esq.

Henry Edward H. Harris, Esq.

W. H. Harrison, Esq.

A. Conning Hartley, Esq., M.D.

F. J. Haverfield, Esq., M.A., LL.D., F.S.A., Camden Professor of Ancient History

Charles C. Hawkins, Esq.

Lawrence Higgins, Esq., J.P.

F. R. Hockliffe, Esq. .
Mrs. Mary A. Hollingsworth

W. Horey, Esq.

Rev. Campbell B. Hulton, M.A.

The Rt. Hon. The Viscount Iveagh, K.P., G.C.V.O., LL.D., F.R.S.

Lt.-Col. Frederick John Josselyn

C. W. Kaye, Esq., M.A.

The Rt. Hon. The Lord Kenyon, K.C.V.O.

John C. Kershaw, Esq.

Graham Charles Lawson, Esq.

E. T. Leeds-Smith, Esq., J.P.

F. Seymour Lloyd, Esq., M.D.

Henry Longuet Longuet-Higgins, Esq.

Walter Thomas Lye, Esq.

John Charles Mason, Esq.

Mrs. Cyril Maude

Joseph Miller, Esq.

The Hon. Algernon H. Mills

W. Gifford Nash, Esq., F.R.C.S.

Clement Buckley Newbold, Esq.

T. Newton, Esq.

The Rt. Hon. The Lord Northcliffe

J. Crewe Orlebar, Esq.

Richard Orlebar, Esq., D.L., J.P.

Sir A. Kerr Butler Osborn, Bart.

Charles G. Pace, Esq., F.R.M.S.

Rev. John Parr, M.A.

C. R. S. Payne, Esq., J.P.

The Rt. Hon. The Viscount Peel

The Hon. William Peel, M.P.

Mrs. Penton

George Phillips, Esq.

Sir Lionel Phillips, Bart., D.L.

Cecil Polhill, Esq.

His Grace The Duke of Portland, K.G., G.C.V.O.

Mrs. Edward Pratt

W. W. G. Prosser, Esq.

Rowland Edmund Prothero, Esq., M.V.O., M.A., F.R.Hist.Soc., \&c.

Capt. Charles Guy Pym, D.L., J.P., F.G.S.

The Rt. Hon. The Earl of Radnor, V.D.

His Excellency The Hon. Whitelaw Reid

Robert Richmond, Esq., J.P.

Lady Roscoe

Charles Julius Ryland, Esq.

Mrs. Rylands

James Saunders, Esq.

Frederick Sharman, Esq.

W. R. Sheldon, Esq., M.A. 


\section{LIST OF SUBSCRIBERS}

The Rt. Hon. The Lord Sherborne

Clement K. Shorter, Esq.

B. Shuttleworth, Esq.

Col. Frank Shuttleworth, J.P.

C. Charnock Smith, Esq., M.R.C.S., L.R.C.P.

F. Gould thorpe Smith, Esq.

H. H. Smith-Carington, Esq., J.P.

His Honour Sir 'Thomas Snagge, K.C.M.G., M.A., LL.D., D.L.

John Tricks Spalding, Esq., J.P.

Miss Stanton

J. Steele-Elliott, Esq.

The Hon. Frederick Strutt

F. W. Styan, Esq.

Lady Sutton

John Taylor, Esq.
Alfred Trapnell, Esq.

Percival Fox Tuckett, Esq.

Charles Robert Wade-Gery, Esq., M.A., J.P.

J. H. Wall, Esq.

William Clarence Watson, Esq., D.L., J.P.

Charles Wells, Esq.

Lady Wernher

Samuel Whitbread, Esq., D.L., J.P.

J. Arnold Whitchurch, Esq.

J. W. Willis-Bund, Esq., M.A., LL.B., F.S.A.

Anthony Henry Wingfield, Esq., J.P.

Alexander Wood, Esq.

H. Wood, Esq., M.A., J.P.

Rev. H. B. Workman, LL.D.

Rev. Paul Williams Wyatt, M.A.

Henry Young, Esq.

\section{PUBLIC AND PRIVATE LIBRARIES}

LONDON

The Athenæum

The Bath Club

Battersea Central Public Library

Bermondsey Public Library

Bishopsgate Institute

The Board of Agriculture

The British Museum

The Carlton Club

Chelsea Public Library

The Conservative Club

The Guildhall Library

Hammersmith Public Library

Hampstead Public Library

The House of Commons Library

The Incorporated Law Society

The Junior Carlton Club

The Junior Naval and Military Club

Kensington Public Library

Lincoln's Inn Library

The London Library

The National Liberal Club

The New University Club

The Reform Club

The Royal Societies' Club

Sion College

The Society of Antiquaries of London

The Surveyors' Institution, Westminster

Tottenham Central Library

'The Travellers' Club

The United University Club

The Victoria and Albert Museum

Westminster Public Library

Westminster and Southland Training College

Dr. Williams' Library

Wimbledon Public Library

\section{The Provinces}

Bedford Arts Club

Bedford County Library

Bedford Grammar School

Bedford Modern School
Birmingham Corporation Reference Library

Bolton Central Reference Library

Bradford Free Public Library

Brighton Public Library

Bristol Public Library

Cambridge Free Library

Cambridge University Library

Cardiff Free Public Library

Cheltenham College

Croydon Public Library

Gloucester Public Library

Hitchin Public Library

Hull Public Library

Kingston-on-Thames Public Library

Leeds Library

Leeds Public Library

Leicester Free Public Library

Leigh (Lancs.) Public Library

Liverpool Public Library

Luton Free Library

Chetham's Library, Manchester

Manchester Free Library

John Rylands Library, Manchester

Victoria University Library, Manchester

Newcastle-on-Tyne Literary and Philosophical Society

Newcastle-on-Tyne Public Library

Northampton Public Library

Nottingham Free Library

Bodley's Library, Oxford

Christchurch, Oxford

New College, Oxford

Oxford Union Society

Preston (Lancs.) Free Library

Reading Free Public Library

Reading University (presented by The Lady Wantage)

Royal Library, Windsor Castle

SCOTLAND

Aberdeen University Library

The Advocates' Library, Edinburgh 


\section{LIST OF SUBSCRIBERS}

Edinburgh Public Library

Edinburgh University Library

The Signet Library, Edinburgh

Glasgow University Library

Mitchell Library (Moir Collection), Glasgow

St. Andrews University Library

\section{IRELAND}

King's Inn Library, Dublin

The National Library of Ireland

Trinity College, Dublin

\section{The Colonies}

Adelaide Public Library, South Australia

New South Wales Public Library, Sydney

Victoria Public Library, Melbourne, Australia

Toronto Public Library, Canada

Toronto University Library, Canada

Victoria Public Library, British Columbia

The General Assembly Library, Wellington, New Zealand

\section{America}

\section{Albany State Library}

Boston (Mass.) Athenæum

Boston (Mass.) Public Library

Brookline (Mass.) Public Library

Cambridge (Mass.) Public Library

Chicago Public Library

Cincinnati Public Library

Columbia University, New York

Connecticut Historical Society

Cornell University Library, Ithaca

Forbes Library, Northampton, Mass.
Harvard University Library

Illinois University Library

Indiana State Library

Iowa State Library

Maine State Library

Massachusetts State Library

Michigan State Library

Newberry Library, Chicago

New Hampshire State Library

Grosvenor Public Library, New York

New York Historical Society

New York Public Library

New York State Library

Ohio State Library

Peabody Institute, Baltimore

Pennsylvania State Library

Philadelphia Free Library

Providence Free Library

The Library of Congress, Washington

Wisconsin State Historical Society

Worcester (Mass.) Free Public Library

Yale University Library

The Continent

Royal Library, Berlin

Royal Library, Copenhagen

Royal Public Library, Dresden

Royal University Library, Göttingen

Lille University Library

Royal Library, Münich

National Library, Paris

Imperial Public Library, St. Petersburg

Royal Library, Stockholm

Upsala University Library 
Printed by

W. H. Sмiтh \& Son,

53-55, Fetter Lane, E.C. 




PLEASE do NOT REMOVE CARDS OR SLIPS FROM THIS POCKET

UNIVERSITY OF TORONTO LIBRARY 
